\title{
UV-pump IR-probe Spectroscopy of Molecules with Time-Resolution reaching the 10-fs Range
}

Kyriaki Kosma

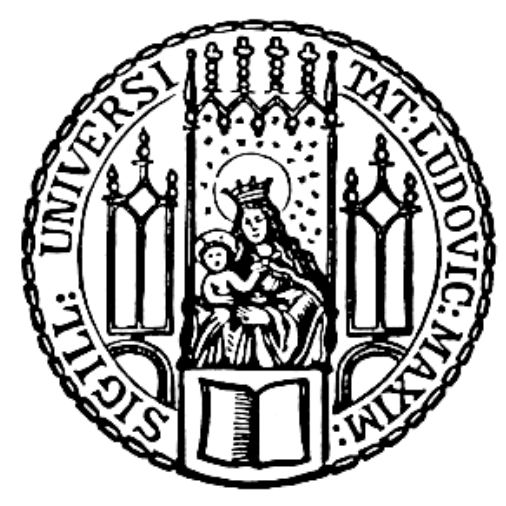

München 2008 



\title{
UV-pump IR-probe Spectroscopy of Molecules with Time-Resolution reaching the 10 -fs Range
}

Kyriaki Kosma

\author{
Dissertation \\ an der Fakultät für Physik \\ der Ludwig-Maximilians-Universität \\ München
}

vorgelegt von

Kyriaki Kosma

aus Thessaloniki, Griechenland

München, 15 Juli 2008 
Erstgutachter: Ferenc Krausz

Zweitgutachter: Wolfgang Domcke

Tag der mündlichen Prüfung: 11-11-2008 


\section{Contents}

Abstract iv

\section{I ntroduction viii}

1. Interaction of atoms with intense laser fields 1

\subsection{Introduction 1}

1.2 Non-linear interaction of light with matter 2

1.2.1 The optical Kerr effect 3

1.2.2 Self-phase modulation 5

1.2.3 Material dispersion and devices for pulse compression 8

1.2.4 Self-focusing 10

1.2.5 Harmonic generation 13

1.3 Temporal characterization of femtosecond pulses 14

2 Ultrashort pulse generation in the IR and UV/ VUV region 16

\subsection{Experimental set-up 16}

\subsection{Supercontinuum generation 17}

2.3 Characterization of the supercontinuum radiation 19

2.3.1 Variation of the input iris 19

2.3.2 Transverse beam profile in the filament region 20

2.3.3Influence of the interaction length 22

2.3.4 Effect of the pulse duration 23

2.3.5 Effect of the focal length 25

2.3.6 Variation of the initial chirp 26

2.3.7 Spatial variation of the supercontinuum spectrum 28

2.3.8 Argon partial pressure 29

2.3.9 Different gases 31

2.4 Pulse duration and compression in the 270 - $320 \mathrm{~nm}$ region of the supercontinuum 31

2.5 Third- and fifth-harmonic generation 35

2.6 Discussion 39

2.7 Conclusions 46

3 Time-resolved spectroscopy 49

\subsection{Introduction 49}

3.2 Pump-probe technique $\mathbf{5 0}$ 


\subsection{Theoretical considerations of the molecular system 51}

3.3.1 The Born-Oppenheimer approximation 51

3.3.2 Beyond the Born-Oppenheimer approximation:

The conical intersection 53

3.3.3 Selection rules in light absorption 56

\subsection{Reaction dynamics $\mathbf{5 8}$}

3.4.1 Deactivation processes 58

3.4.2 The pathway approach 59

3.4.3 Description of the dynamics by rate equations and periodic modulations 60

4 Dynamics of internal conversion and isomerization of ethylene and ethylene- $d_{4} 65$

\subsection{Experimental 65}

4.2 Background 66

4.3 Results and analysis 69

4.4 Discussion 77

4.4.1 Assignments of the lifetimes and oscillations 77

4.4.1.1 The Franck-Condon region 78

4.4.1.2 Departure from $S_{1} 80$

4.4.1.3 Ground-state processes 83

4.4.1.4 Results of spectroscopic relevance 86

4.4.2 Ultrafast dynamics and implication for the potentials 88

\subsection{Conclusions 91}

5 The ring opening of cyclohexadiene 93

\subsection{Experimental 93}

5.2 Background 93

5.3 Results and analysis 96

5.4 Discussion 98

5.4.1 Assignments of the lifetimes and oscillations 99

5.4.2 The reaction path 103

\subsection{Conclusions 107}

6 The dynamics of the dissociation of $\operatorname{Cr}(\mathrm{CO})_{6} 110$

\subsection{Experimental 110}

6.2 Background 111 
6.3 Results and analysis 113

6.3.1 Dynamics of $\mathrm{Cr}(\mathrm{CO})_{6}$ excited at $270 \mathrm{~nm} 113$

6.3.2 Dynamics of $\mathrm{Cr}(\mathrm{CO})_{6}$ excited at 318 and $345 \mathrm{~nm} 117$

\subsection{Discussion 118}

6.4.1 Assignments of the lifetimes and oscillations 118

6.4.2 The reaction path of the dissociation 121

6.5 Conclusions 126

Appendix 129

Summary 133

References 137

Acknowledgements 151 


\section{Abstract}

This work investigates the real-time dynamics of some fundamental molecules in the gas phase during light-induced chemical reactions, by pushing the time resolution down to about 10 fs (30 fs initially). These reactions are the isomerization of ethylene $\left(\mathrm{C}_{2} \mathrm{H}_{4}\right)$, the ring opening of cyclohexadiene $\left(\mathrm{C}_{6} \mathrm{H}_{8}\right)$ and the dissociation of chromium hexacarbonyl $\left(\mathrm{Cr}(\mathrm{CO})_{6}\right)$.

These molecules were expected - and confirmed - to exhibit several consecutive very fast processes. In addition, high frequency coherent oscillations were searched and indeed resolved; their assignment to vibrations allows conclusions on geometrical distortions of the molecule during the processes, on directions of slopes (forces) on the potential and on the path of the wave packet. The time-resolution is now better by a factor of 7 (in the near UV) to 15 (at $160 \mathrm{~nm}$ ) than in the previous best cases. This required the development of methods to generate short UV pulses, which were not available, in contrast to the $800-\mathrm{nm}$ region. The developed light sources are based on (1) supercontinuum generation and (2) third- and fifthharmonic generation, both in argon, starting from 10-fs Ti:sapphire laser pulses.

Chapters 1 and 2 report on phenomena during propagation of very short (spectrally broad) and intense femtosecond laser pulses through nonlinear media (mainly gaseous argon) and on experimental methods - exploiting self-phase modulation and harmonic generation - for producing ultrashort pulses in the region of the fundamental $(800 \mathrm{~nm})$ of a commercial Ti:sapphire laser system, as well as outside this spectral range, in the UV $(250-350 \mathrm{~nm})$ and vacuum UV (162 nm). In a first step, 10-fs at 810-nm were achieved by self-phase modulation in argon in free propagation (with self-focusing), combined with chirped-mirror compression (see also [10] from list of publications, p. 153) ${ }^{1}$. Refocusing these pulses into argon produced a supercontinuum reaching down to $250 \mathrm{~nm}$ whose properties (e.g. cut-off wavelength and duration) were investigated $[10,4]$. The supercontinuum allowed cutting out radiation in the UV with tunable wavelength and pulse duration of $30 \mathrm{fs}$ after propagation in air [9] and $10 \mathrm{fs}$ with a vacuum beam path [6]. With a much shorter argon cell, third- and fifth-harmonic pulses (270 and $162 \mathrm{~nm}$ ) were obtained with durations ( 10 fs) slightly shorter than the 810-nm fundamental pulses used (12 fs) [6, 3] (initially $30 \mathrm{fs}$ for the TH [9]). Although further shortening

\footnotetext{
${ }^{1}$ All the references contained in the abstract are listed on page 153.
} 
seems feasible, these pulses were first applied to pump-probe spectroscopy before any further optimization.

Chapter 3 first presents an introductory theoretical background and the framework, on which the analysis and explanation of the time-resolved findings are based. Chapters 4 to 6 then describe and discuss the results of the implementation of the ultrashort pulses in the pump-probe experiments (with 810 -nm-multiphoton ionization for probing), by which the dynamical processes in the mentioned molecules are monitored in time; lifetimes as short as 12 fs could be found. With the help of the time constants, relative energies and coherent oscillations (vibrations), detailed conclusions on the reaction coordinates and geometrical structures of important points on the potential energy surfaces could be deduced. For example in ethylene five time constants and four excited-state vibrations were found for both $\mathrm{C}_{2} \mathrm{H}_{4}$ and $\mathrm{C}_{2} \mathrm{D}_{4}[2]$; they indicate that the initial motion involves $\mathrm{CC}$ twist and stretch and the conical intersection on transition to the ground state also involves $\mathrm{H}$-migration. In $\mathrm{C}_{6} \mathrm{H}_{8}$ the initial acceleration is towards the Franck-Condon active modes (certainly a general rule), followed by an antisymmetric distortion [1]; but the ring-opening is only half completed in the lowest excited state. In $\mathrm{Cr}(\mathrm{CO})_{6}$ (where tunable pump radiation was used) the different initial states are depleted not via an $\mathrm{S}_{1}$ but via a common dissociative state on separate paths: the paths only merge in the primary product $\mathrm{Cr}(\mathrm{CO})_{5}$ in its $\mathrm{S}_{1}$ state [5]. No triplet state is involved in contrast to the previous belief. 


\section{Zusammenfassung}

Diese Arbeit untersucht die Dynamik einiger fundamentaler Moleküle in der Gasphase während lichtinduzierter chemischer Reaktionen mit verbesserter Zeitauflösung bis etwa 10 fs (anfangs $30 \mathrm{fs}$ ). Diese Reaktionen sind die Isomerisation von Ethylen $\left(\mathrm{C}_{2} \mathrm{H}_{4}\right)$, die Ringöffnung von Cyclohexadien $\left(\mathrm{C}_{6} \mathrm{H}_{8}\right)$ und die Dissoziation von $\mathrm{Cr}(\mathrm{CO})_{6}$.

Es wurde erwartet - und bestätigt - dass diese Moleküle mehrere aufeinanderfolgende, sehr schnelle Prozesse durchlaufen. Zusätzlich wurden hochfrequente kohärente Oszillationen gesucht und tatsächlich aufgelöst. Deren Zuordnung zu Schwingungen erlaubt Rückschlüsse auf geometrische Verformungen des Moleküls während des Prozesses, auf Richtungen von Potentialgefällen (Kräften) und auf den Weg des Wellenpakets. Die Zeitauflösung, die in dieser Arbeit erreicht wurde, ist um einen Faktor 7 (im nahen UV) bis 15 (160 nm) besser als bisherige Bestwerte. Dazu war es notwendig, Methoden zur Erzeugung kurzer UV-Pulse zu entwickeln, welche - im Gegensatz zum 800-nm-Gebiet - nicht verfügbar waren. Die entwickelten Lichtquellen basieren auf (1) Erzeugung eines Superkontinuums (SC) und (2) Erzeugung der dritten und fünten Harmonischen, jeweils in Argon, ausgehend von einem 10-fs Ti:Saphir-Laserpuls.

Kapitel 1 und 2 beschreiben die Ausbreitung von sehr kurzen, spektral breiten und intensiven fs-Laserpulsen durch nichtlineare Medien (hauptsächlich gasförmiges Argon) und experimentelle Methoden zur Erzeugung ultrakurzer Pulse im Bereich der Fundamentalen (800 $\mathrm{nm}$ ) eines kommerziellen Ti:Saphir-Lasersystems sowie außerhalb dieses Spektralbereichs, im UV (250 - $350 \mathrm{~nm})$ und Vakuum-UV (160 nm); diese Methoden basieren auf Selbst-PhasenModulation (SPM) und Harmonischen-Erzeugung. Im ersten Schritt wurden 10-fs-Pulse bei 810-nm mit SPM und Selbst-Fokussierung in Ar, kombiniert mit einem chirped-mirrorKompressor, erreicht ([10] s. 153). Refokussierung dieser Pulse in Ar erzeugt ein SCSpektrum bis $250 \mathrm{~nm}$, dessen Eigenschaften (z.B. Abbruch-Wellenlänge und Dauer) untersucht wurden $[10,4]$. Das SC ermöglichte, Strahlung im UV mit abstimmbarer Wellenlänge und Pulsdauer von 30 fs nach Ausbreitung in Luft [9] und 10 fs in Vakuum herauszuschneiden [6]. Mit einer viel kürzeren Ar-Zelle wurden Pulse bei der dritten und fünften Harmonischen erreicht, mit einer Dauer (10 fs) knapp unter der der verwendeten 810-nm Pulse (12 fs) [6, 3] (anfangs 30 fs für die dritte Harmonische). Obwohl eine weitere Verkürzung der Pulse 
möglich scheint, wurden diese für Pump-Abfrage-Spektroskopie ohne weitere Optimierung verwendet.

Kapitel 3 präsentiert zuerst einen einführenden theoretischen Hintergrund und den Rahmen, auf dem die Analyse und die Erklärung der zeitaufgelösten Ergebnisse beruhen. Kapitel 4 bis 6 beschreiben und diskutieren die Ergebnisse der Anwendung der ultrakurzen Pulse in den Pump-Abfrage-Experimenten (mit 810-nm-Multiphoton-Ionisation als Abfrage), mit denen die Dynamik der erwähnten Moleküle in Echtzeit verfolgt werden; Lebensdauern bis herunter zu 12 fs wurden beobachtet. Detaillierte Schlüsse auf die Reaktionskoordinaten und die geometrischen Strukturen wichtiger Punkte auf dem Potential konnten mit Hilfe von Zeitkonstanten, relativen Energien und kohärenten Oszillationen gezogen werden. Zum Beispiel wurden bei Ethylen fünf Zeitkonstanten und vier Schwingungen im angeregten Zustand sowohl für $\mathrm{C}_{2} \mathrm{H}_{4}$ wie auch für $\mathrm{C}_{2} \mathrm{D}_{4}$ gefunden [2]; sie zeigen, dass an der Anfangsbewegung $\mathrm{CC}$-Verdrillung und -Streckung beteiligt sind und dass beim Übergang in den Grundzustand durch den konischen Schnitt auch H-Wanderung mitwirkt. Bei $\mathrm{C}_{6} \mathrm{H}_{8}$ geht die Anfangsbeschleunigung in Richtung Franck-Condon-aktiver Koordinaten (sicherlich eine allgemeine Regel), gefolgt von einer antisymmetrischen Verzerrung [1]; aber die Ringöffnung ist im niedrigsten angeregten Zustand nur zur Hälfte abgelaufen. In (CrCO) 6 (bei dem abstimmbare Pumpstrahlung verwendet wurde) werden die verschiedenen Anfangszustände nicht über einen $\mathrm{S}_{1}$ entleert, sondern über einen gemeinsamen dissoziativen Zustand auf getrennten Wegen: die Wege laufen erst im Primärprodukt $\mathrm{Cr}(\mathrm{CO})_{5}$ im $\mathrm{S}_{1}-Z$ ustand zusammen [5]. Kein Triplett-Zustand ist beteiligt, im Gegensatz zur früheren Meinung. 


\section{Introduction}

Monitoring the motion of the electrons and nuclei and their behaviour after being disturbed from their state of equilibrium is very challenging and fascinating. It is nowadays well established that most photo-induced transformations of matter, such as photo-chemical reactions, are completed within some tens to hundreds of femtoseconds, with partial steps taking place only in few femtoseconds. The purpose of this work is the microscopic real-time analysis of the dynamics of gas-phase molecules after photoexcitation, with a time resolution improved by about an order of magnitude. The dynamics can be monitored with the help of the so called pump-probe experiments. The reactions investigated are the isomerization of ethylene $\left(\mathrm{C}_{2} \mathrm{H}_{4}\right)$, the ring opening of cyclohexadiene $\left(\mathrm{C}_{6} \mathrm{H}_{8}\right)$ and the dissociation of chromium hexacarbonyl $\left(\mathrm{Cr}(\mathrm{CO})_{6}\right)$. This choice is also motivated by some fundamental points regarding the chemistry of these molecules, which have been the matter of controversy among scientists for many years.

Pump-probe (time-resolved) spectroscopy encompasses a powerful set of techniques for probing and characterizing the electronic and structural properties of short-lived excited states (transient states) of photochemically/photophysically relevant molecules. This is possible with the help of laser pulses, whose durations are short enough for the desired time resolution: a pump pulse prepares the system in an excited state and after a variable time delay a second laser pulse (probe) interacts with the molecule. This interaction depends on the instantaneous location of the latter on the excited potential energy surface (PES). After the first extension into the femtosecond domain by A. H. Zewail ([1] on p. xi) ${ }^{2}$ several pump-probe techniques have been developed for the investigation of nuclear dynamics, which differ in the transient signal observed. Most widespread are those applying transient fluorescence or absorption (see [2] and references therein) and transient ionization (including transient photoelectron spectroscopy), as well as time-resolved diffraction, with which structural changes of matter can be probed. Furthermore, besides molecules in the gas phase, liquids, bulk materials and surfaces have successfully been studied [2]. More recently electron dynamics could be investigated in Auger processes in atoms in a pump-probe experiment with attosecond time resolution [3], observing ions produced via tunnelling ionization by a NIR probe pulse.

\footnotetext{
${ }^{2}$ All the references contained in the introduction are listed on page xi.
} 
In this work pump radiation in the UV initiates the reaction of the molecule under consideration; probing is done by ionizing the molecules with an $810-\mathrm{nm}$ pulse, detecting the ion yields mass selectively in a time-of-flight mass-spectrometer. One advantage of using transient ionization is that it gives rise to many ion signals that can differ in their temporal behaviour, thus revealing more details about the system under investigation than the other techniques, where only one transient signal is usually recorded. Moreover, it is important that no selection rule prevents the detection of states that are far from the initially excited region, in contrast to optical spectroscopy, where some states can be "dark", i.e. not accessible by a vertical transition from the ground surface. The method thus allows one to monitor the complete path of the molecule over the potential surfaces, from the initially excited ("Franck-Condon") region via states far displaced from it, down to the ground state of the product(s).

Time resolution has so far been limited by the duration of the pulses in the UV (even more so in the vacuum UV), where small molecules have their first absorption band. A first goal in this work was therefore the development of sources that provide pulses in the 10-fs range in this spectral region, if possible of tunable wavelength. In contrast, in the region of the Ti:sapphire fundamental $(800 \mathrm{~nm}$, probe wavelength) techniques for pulse shortening were available. The most common one uses nonlinear broadening about the central wavelength by self-phase modulation (SPM) and subsequent compression with advanced multilayer optics [4]. This is achieved in gas-filled capillaries [5], which provide the required length for interaction with the nonlinear medium at high intensity, and recently also in gas filaments, as was demonstrated by Hauri et al. [6]. Tunable femtosecond pulses in the UV have so far been produced by the following techniques, achieving usually high energies (a few $\mu \mathrm{J}$ ) (see [7] and references therein): (1) an optic parametric amplifier (OPA) followed by nonlinear frequency conversion. But the phase-matching bandwidth lengthens the pulses to $70 \mathrm{fs}$ around $250 \mathrm{~nm}$ and $150 \mathrm{fs}$ around $200 \mathrm{~nm}$; (2) a non-collinear OPA combined with achromatic frequency doubling. With this method pulses down to $7 \mathrm{fs}$ could be obtained, but so far they are limited to a wavelength $\geq 270 \mathrm{~nm}$; (3) four-wave mixing of the type $\omega_{1}+\omega_{1}-\omega_{\mathrm{IR}}$ in gas-filled capillaries combined with a prism compressor, obtaining wavelengths $\lambda \geq 233 \mathrm{~nm}$ and pulse durations $\geq$ 31 fs; (4) cross-phase modulation of a weak tunable pulse with a strong short fundamental followed by a prism compressor; (5) four-wave mixing in free propagation (filaments) in gases. The wavelengths obtained have been so far limited to the visible region of the spectrum. 
In this work supercontinuum (SC) and third- and fifth-harmonic generation in gaseous $\mathrm{Ar}$ were the two methods employed for generation of light in the UV region. In the first case, the tunability and the cut-off wavelength, the conversion efficiency, the short pulse duration and the pulse-to-pulse and pointing stability of the generated radiation proved valuable and reliable for using the SC experimental set-up on a daily basis for some of the measurements; it provides a relatively wide wavelength region $(250-800 \mathrm{~nm})$ and avoids possible challenges in alignment that might arise in the previous methods. The wavelengths actually applied in time-resolved measurements covered the UV range 270 - $340 \mathrm{~nm}$ (with durations $10 \mathrm{fs}$ or - in an initial stage of development - $30 \mathrm{fs}$ ) and were used to study the dynamics of several excited states of $\mathrm{Cr}(\mathrm{CO})_{6}$. From third- and fifth-harmonic generation the wavelengths 270 and $162 \mathrm{~nm}$ were obtained (with durations $10 \mathrm{fs}$ or initially $30 \mathrm{fs}$ ). This radiation served as the pump pulse for exciting the CHD molecules and $\mathrm{C}_{2} \mathrm{H}_{4}$ and $\mathrm{C}_{2} \mathrm{D}_{4}$, respectively. Due to the shorter durations achieved in comparison with previous pump-probe experiments (e.g. in ethylene, Chapter 4) and the higher time resolution many more details and insight concerning the dynamics could be obtained.

With the high time resolution, we were able to answer some still open questions concerning the reactions studied. In the isomerization of ethylene (excited at 162-nm) there has been a lot of controversy regarding the geometry of the conical intersection of the first excited $\left(\mathrm{S}_{1}\right)$ with the ground $\left(\mathrm{S}_{0}\right)$ state. Some theoretical works favour the pyramidalized configuration $[8$, 9] and some others H-migration [10]. From the simultaneous formation of $\mathrm{CHCH}_{3}$ and other observations we conclude that the $\mathrm{S}_{1} / \mathrm{S}_{0}$ conical intersection (CI) (the branching region for the path) involves partial $\mathrm{H}$-migartion and a structure with an $\mathrm{H}$ bridge. Moreover, the lifetimes and oscillations that could be resolved for both $\mathrm{C}_{2} \mathrm{H}_{4}$ and $\mathrm{C}_{2} \mathrm{D}_{4}$ provide information and details indicating the complete path of the reaction, from the Franck-Condon (FC) region of the $\pi \pi^{*}$ state down to the ground state $\mathrm{S}_{0}$.

Cyclohexadiene has been studied extensively and its ring-opening reaction path after excitation at $270 \mathrm{~nm}$ is probably known in the most detailed way among the three molecules studied in this work. This reaction is a prototype of the large class of pericyclic reactions. With the better time-resolution, oscillations could now be resolved that were assigned to vibrations, thus confirming the previously suggested reaction path for the opening of the ring, including details such as changes in the direction of motion on the potentials. 
A prototype of organometallic photochemistry is the photodissociation of metal carbonyls. The study of their dynamics has many implications for other metal complexes. A very controversial point in the $\mathrm{CO}$ elimination of $\mathrm{Cr}(\mathrm{CO})_{6}$ was whether or not there are any triplet states involved. Before high-level calculations $[11,12,13]$ were feasible with complexes such as $\mathrm{Cr}(\mathrm{CO})_{6}$, it was assumed [14] (and considered established in textbooks, e.g. [15]) that the weak longer-wavelength transitions lead to triplet states (instead of the symmetry-forbidden singlet states). The similar reaction paths found in our pump-probe experiment starting from different initial excited states imply that no triplet state is involved in the dynamics of the dissociation, neither in the form of intersystem crossing from an initially excited singlet state, nor by exciting directly a triplet state in the intact molecule. This is an important conclusion, since triplet products would radically differ in their chemical reactions from the corresponding singlet molecules. $\mathrm{Cr}(\mathrm{CO})_{6}$ also suggested to check a general assumption in photophysics and photochemistry, the Kasha rule, according to which the paths from different excited states all merge in the lowest excited state, before the actual process (fluorescence or reaction) takes place.

\section{References}

1. Zewail, A.H., Laser femtochemistry. Science, 1988. 242: p. 1645.

2. Diels, J.C. and W. Rudolph, Ultrashort Laser Pulse Phenomena. second ed. 2006: Elsevier.

3. M. Uiberacker, Th. Uphues, M. Schultze, A. J. Verhoef, V. Yakovlev, M. F. Kling, J. Rauschenberger, N. M. Kabachnik, H. Schröder, M. Lezius, K. L. Kompa, H.-G. Muller, M. J. J. Vrakking, S. Hendel, U. Kleineberg, U. Heinzmann, M. Drescher, F. Krausz, Attosecond real-time observation of electron tunnelling in atoms. Nature, 2007. 446: p. 627.

4. Brabec, T. and F. Krausz, Intense few-cycle laser fields: Frontiers of nonlinear optics. Review of Modern Physics, 2000. 72: p. 545.

5. Nisoli, M., De Silvestri, S. and Svelto, O., Generation of high energy 10 fs pulses by a new pulse compression technique. Appl. Phys. Lett., 1996. 68: p. 2793.

6. Hauri, C.P., et al., Generation of intense, carrier-envelope phase-locked few-cycle laser pulses through filamentation. Applied Physics B, 2004. 79: p. 673.

7. Trushin, S.A., et al., Widely tunable ultraviolet sub-30 fs pulses from supercontinuum for transient spectroscopy. Applied Physics B, 2006. 85: p. 1-5. 
8. Ben-Nun, M. and T.J. Martínez, Ab initio molecular dynamics study of cis-trans isomerization in ethylene. Chemical Physics Letters, 1998. 298: p. 57-65.

9. Ben-Nun, M., J. Quenneville, and T.J. Martínez, Ab initio multiple spawning: Photochemistry from first principles quantum molecular dynamics. Journal of Physical Chemistry A, 2000. 104: p. 5161-5175.

10. Barbatti, M., M. Ruckenbauer, and H. Lischka, The photodynamics of ethylene: A surface-hopping study on structural aspects. Journal of Chemical Physics, 2005. 122: p. 174307/1-9.

11. Rosa, A., et al., Electronic spectra of $M(C O)_{6}(M=C r, M o, W)$ revisited by a relativistic TDDFT approach. Journal of the American Chemical Society, 1999. 121: p. 10356-1065.

12. Pierloot, K., E. Tsokos, and L.G. Vanquickenborne, Optical spectra of $\mathrm{Ni}(\mathrm{CO})_{4}$ and $\mathrm{Cr}(\mathrm{CO})_{6}$ revisited. Journal of Physical Chemistry, 1996. 100: p. 16545-16550.

13. Pollak, C., A. Rosa, and E.J. Baerends, $\mathrm{Cr}$-CO photodissociation in $\mathrm{Cr}(\mathrm{CO})_{6}$ : Reassessment of the role of ligand-field excited states in the photochemical dissociation of metal-ligand bonds. Journal of the American Chemical Society, 1997. 119: p. 7324-7329.

14. Beach, N.A. and H.B. Gray, Electronic structures of metal hexacarbonyls. Journal of the American Chemical Society, 1968. 90: p. 5713-5721.

15. Geoffroy, G.L. and M.S. Wrighton, Organometallic Photochemistry. 1979, New York: Academic Press. 


\section{1}

\section{Interaction of atoms with intense laser fields}

\subsection{I ntroduction}

Standard techniques for producing ultrashort laser pulses with duration of few femtoseconds are based on self-phase modulation (SPM). SPM takes place during propagation in a nonlin-

ear material and results from third $\operatorname{order}\left(\chi^{(3)}\right)$ nonlinearities; it arises from the optical Kerr effect in combination with the temporal intensity profile. The resulting positively chirped pulses have a broadened spectrum, which is subsequently exploited for compressing the pulse to near its Fourier-limit, thus shortening its duration down to few cycles. This compression is feasible by common techniques that profit from the negative group delay dispersion of chirped mirrors, pairs of prisms or gratings. If higher powers are desired, one can only use gases as the nonlinear medium. In this case SPM requires long path lengths in the medium, which are usually provided by light waveguides or gas-filled capillaries; this requires a very good and reproducible alignment and pointing stability to avoid damage of the capillary.

Recently Hauri et al. [9] demonstrated an alternative, based on self-guiding in a gas: They focused their pulses (43 fs, $0.84 \mathrm{~mJ}, 800 \mathrm{~nm}$ ) into an argon-filled cell, compressed the resulting spectrally broadened radiation by chirped mirrors to 10.5 fs and by repeating the process obtained pulses with duration of 5.7 fs. The necessary interaction length with high and stable intensity, in this case, was provided by self-guiding, caused by self-focusing (filamentation). This phenomenon is due to a balance between self-focusing (which arises from the transverse beam intensity profile and the intensity-dependent refractive index caused by the Kerr effect) and defocusing (caused by the plasma generated on the axis due to the high laser intensity). 
Trying the method of Hauri the possibility of producing short pulses was confirmed: As in [9] we obtained pulses of $\approx 10 \mathrm{fs}$ at $810 \mathrm{~nm}$ in the first step (first argon cell and chirped mirrors [1]). In addition, we discovered that the spectrum of the pulses is very broad after the second argon cell, exhibiting a pedestal that reaches down to $250 \mathrm{~nm}$ in the UV region [1]. The emerging white light laser pulses promised to be a source of light of tunable short pulses in the UV. This was indeed recently demonstrated, with application to time-resolved spectroscopy in the region $270-360 \mathrm{~nm}$ [3]. The mechanism and conditions that produce and affect the supercontinuum spectrum were therefore investigated in more detail. Moreover, the possibility of compressing the UV part of the white-light radiation, as well as other methods to obtain short UV pulses were investigated (third- and fifth-harmonic generation in gases), since it is of great importance to obtain ultrashort pulses in this wavelength region, where many molecules have their first absorption band, in order to perform high-resolution time-resolved studies; indeed, sub-10 fs pulses could be generated around $290 \mathrm{~nm}$ by means of supercontinuum generation [4]. Similarly, pulses of $\sim 10 \mathrm{fs}$ are obtained in the UV and VUV region by means of third- and fifth-harmonic generation [4, 5].

\subsection{Non-linear interaction of light with matter}

Nonlinearity is a property that a material exhibits when intense light is transmitted through it. An optical field polarizes the medium by altering the relative positions of the charges (mainly the electrons), which respond to the applied electric field; the medium then in turn modifies the original field as this propagates through it and changes its phase and - in the presence of a nonlinearity - also adds new frequency components. The electric field induces dipole moments and the quantity of the dipole moment per unit volume is called polarization density $\boldsymbol{P}$; $\boldsymbol{P}$ is therefore a measure of the medium response to an external electric field, which causes $\boldsymbol{P}$ to oscillate under its influence.

The mathematical relation between the vectors $\boldsymbol{P}(\boldsymbol{r}, t)$ and the applied electric field $\boldsymbol{E}(\boldsymbol{r}, t)$ is $\boldsymbol{P}=\varepsilon_{0}\left(\chi^{(1)} \cdot \boldsymbol{E}+\chi^{(2)}: \boldsymbol{E} \boldsymbol{E}+\chi^{(3)} \vdots \boldsymbol{E} \boldsymbol{E} \boldsymbol{E}+\ldots\right)=\boldsymbol{P}^{L}+\boldsymbol{P}^{N L}$, which describes the optical properties of the system; $\varepsilon_{0}$ is the permittivity of free space, $\chi^{(1)}, \chi^{(2)}, \chi^{(3)} \ldots$ are the electric susceptibilities of the medium of first, second, third $\ldots$ order and $\boldsymbol{P}^{L}, \boldsymbol{P}^{N L}$ the linear and nonlinear polarizations, respectively. The medium is said to be nonlinear, when the higher-order terms are not negligible compared to the linear term. The susceptibilities $\chi^{(n)}$ describe the lin- 
ear $\left(\chi^{(1)}\right)$ and nonlinear properties of a medium, as well as the corresponding linear and nonlinear optical effects that arise when the external electric field is applied. They depend on the structure and the properties of the propagation medium. In isotropic media $\chi^{(n)}$ are scalar quantities that are related to the polarizabilities $\alpha^{(n)}$ of the corresponding order according to the relation $\chi^{(n)}=\alpha^{(n)} \rho / \varepsilon_{0}$, if the number density $\rho$ of the material is small such as for gases. In addition, in isotropic media, $\chi^{(2)}$ (and the higher $\chi^{(n)}$ with even $n$ ) vanish, so that $\chi^{(3)}$ is the lowest-order nonlinearity for gases, e.g. Ar that is mainly used in these experiments.

The above expression for $\boldsymbol{P}$ constitutes the perturbative description of the nonlinear response of matter to an applied electric field. It is a good approximation for intensities up to around $10^{13} \mathrm{~W} / \mathrm{cm}^{2}$ [10], because it corresponds to a field amplitude of $0.87 \mathrm{~V} / \AA$, which is small enough compared to the atomic unit of field strength $(60 \mathrm{~V} / \AA)$. In the present work the maximum intensity reached was $\approx 5 \times 10^{13} \mathrm{~W} / \mathrm{cm}^{2}$.

\subsubsection{The optical Kerr effect}

The optical effect most interesting for SPM in gases is the optical Kerr effect, which is based on the third-order nonlinear response of matter to a strong applied electric field [11, 12]. As mentioned, for isotropic media such as gases, $\chi^{(3)}$ is the lowest-order nonlinearity and is scalar. Therefore, $\boldsymbol{P}^{N L}$ is parallel to $\boldsymbol{E}$ (which is transverse to the propagation direction $z$ ). The relation that connects the $j$-th component of the third-order polarization $\boldsymbol{P}^{(3)}, P_{j}^{(3)}=\varepsilon_{0} \chi^{(3)} E_{j}^{3}$, with the $j$-th component of the electric field $\boldsymbol{E}$ in the general case, where the electric field is the sum of a number of monochromatic waves $E_{j}(z, t)=\sum_{n} \frac{1}{2} E_{j}\left(\omega_{n}\right) \exp \left[-i \omega_{n} t\right]$, is

$$
P_{j}^{(3)}=\frac{1}{8} \varepsilon_{0} \sum_{m, n, s} \chi^{(3)}\left(\omega_{m}, \omega_{n}, \omega_{s}\right) E_{j}\left(\omega_{m}\right) E_{j}\left(\omega_{n}\right) E_{j}\left(\omega_{s}\right) \exp \left[-i\left(\omega_{m}+\omega_{n}+\omega_{s}\right) t\right]
$$

with $E_{j}\left(\omega_{n}\right)=F_{j}\left(\omega_{n}\right) \exp \left[i k_{n} z\right]$ being the complex amplitude and $F_{j}\left(\omega_{n}\right)$ the complex envelope of the wave of frequency $\omega_{n}$ and wave vector $k_{n}$. The sum extends over positive and negative values of $m, n, s$ defining $\omega_{-n}=-\omega_{n}$ and $E_{j}\left(\omega_{-n}\right)=E_{j}^{*}\left(\omega_{n}\right)$ so that the electric field and the polarization wave are real quantities. 
Equation (1.2.1.1) shows that $\boldsymbol{P}^{(3)}$ in general consists of components with all frequency combinations $\omega_{m}+\omega_{n}+\omega_{s}$ and therefore not only a third-harmonic ( $\left.3 \omega\right)$ component, but also one at the fundamental $\omega$. Taking for example a monochromatic wave of frequency $\omega$, polarized along the $x$ axis and propagating along the $z$ axis $\boldsymbol{E}(z, t)=\boldsymbol{x}^{0}(1 / 2) E(\omega) \exp [-i \omega t]+$ c.c. and considering only the term that mixes $\omega, \omega,-\omega$ in (1.2.1), we get the $\omega$-component of the nonlinear polarization $\boldsymbol{P}^{(3)}(\omega)=\boldsymbol{x}^{0} 3 \varepsilon_{0} \chi^{(3)}|E(\omega)|^{2} E(\omega) \exp [-i \omega t] / 8+$ c.c. [12]. This thirdorder induced polarization wave has the same frequency $\omega$ as the driving electromagnetic wave. If the linear and this third-order polarization are combined, one obtains

$$
\boldsymbol{P}(\boldsymbol{r}, t)=\boldsymbol{P}^{(1)}(\boldsymbol{r}, t)+\boldsymbol{P}^{(3)}(\boldsymbol{r}, t)=\boldsymbol{x}^{0} \varepsilon_{0}\left(\frac{1}{2} \chi^{(1)}+\frac{3}{8} \chi^{(3)}|E(\omega)|^{2}\right) E(\omega) \exp [-i \omega t]+\text { c.c. }
$$

where $\chi^{(1)}$ is the first order susceptibility that couples the linear polarization $\boldsymbol{P}^{(L)}$ with the electric field.

The expression $\chi^{(1)}+3 \chi^{(3)}|E|^{2} / 4$ can be treated on the whole as susceptibility, which in general is complex; the real and imaginary part of the refractive index $n$ can be derived from $n^{2}=1+\chi=1+\chi^{(1)}+\frac{3}{4} \chi^{(3)}|E|^{2}=\left(n_{0}+\Delta n+i \alpha\right)^{2} \approx n_{0}^{2}+2 n_{0} \Delta n+i 2 n_{0} \alpha \quad[12] . n_{0}^{2}=1+\chi^{(1)}$ is the linear index of refraction, $\Delta n=3 \operatorname{Re}\left(\chi^{(3)}\right)|E|^{2} / 8 n_{0}$ is the correction to the linear refractive index and the coefficient $\alpha$ (which is related to the imaginary part of $\chi^{(3)}$ ) is directly connected to two-photon absorption by the nonlinear medium; it is neglected in the rest of this work. The above correction to the refractive index can be expressed in terms of the light intensity $I$ as $\Delta n=n_{2} n_{0} \varepsilon_{0} c|E|^{2} / 2=n_{2} I$, where $n_{2}=3 \operatorname{Re}\left(\chi^{(3)}\right) / 4 n_{0}^{2} \varepsilon_{0} c$ is the nonlinear index or Kerr coefficient that is usually $n_{2}>0$. The change $\Delta n$ to the linear refractive index $n_{0}$ due to the strong light intensity is known as the optical Kerr effect; the dc Kerr effect is the change of $n_{0}$ by a dc field. The unit of $n_{2}$ is $\mathrm{cm}^{2} / \mathrm{W}$ and its order of magnitude is $10^{-16}$ to $10^{-14}$ for glasses, $10^{-10}$ to $10^{-8}$ for organic materials, $10^{-10}$ to $10^{-2} \mathrm{~cm}^{2} / \mathrm{W}$ for semiconductors ([11], chap. 19.3) and $10^{-20}$ to $10^{-18}$ for gases.

After a distance $L$ of light propagation in the nonlinear medium the phase of the light pulse will be $\phi=k L-\omega t=\left(n_{0}+\Delta n\right) \omega L / c-\omega t$. Therefore the intensity-induced phase shift that will 
have been accumulated after propagation of length $L$ will be $\Delta \phi=\Delta n \omega L / c=2 \pi n_{2} I L / \lambda_{0}$. For large-band gap materials, which have no absorption at low frequencies, with intensity levels below the damage threshold such as $I=10^{12} \mathrm{~W} / \mathrm{cm}^{2}$ and $n_{2}=10^{-16} \mathrm{~cm}^{2} / \mathrm{W}$, one obtains $\Delta n=$ $10^{-4}$. Despite the very small correction $\Delta n$, the phase shift $\Delta \phi$ can be substantial, due to its dependence on the length of propagation. For $\lambda=800 \mathrm{~nm}$ and $L=8 \mathrm{~mm}, \Delta \phi=2 \pi$. For gaseous argon at atmospheric pressure $n_{2}=1.4 \times 10^{-19} \mathrm{~cm}^{2} / \mathrm{W}[13]$ and with $I=5 \times 10^{13} \mathrm{~W} / \mathrm{cm}^{2}$ (an intensity still below the optical breakdown) the refractive index changes by $\Delta n=7 \times 10^{-6}$, which should be compared with the linear refractive index $n_{0}=1+2.8 \times 10^{-4}$. A path length of $L=11 \mathrm{~cm}$ is then required to obtain $\Delta \phi=2 \pi$.

Apart from the dependence of the phase shift on the propagation distance, $\Delta \phi$ also follows the temporary variation of the light intensity $I$. In Gaussian pulses travelling along the $z$ axis, the intensity $I$ varies as a Gaussian function both with time and the transverse coordinates $x, y$. As a result, the temporal and the spatial variation of $I$ introduce corresponding variations in $\Delta \phi$, which in turn give rise to two phenomena, self-phase modulation [14] and self-focusing $[14,15]$. Since they are caused by the term $n_{2} I$, they are both consequences of the optical Kerr effect.

\subsubsection{Self-phase modulation}

Assuming in a first step a laser pulse of uniform transverse profile, only the temporal variation of the intensity $I$ will initially be considered. A beam of a Gaussian temporal profile $I(t)=I_{0} \exp \left[-(t / \tau)^{2}\right]$ propagating through the Kerr medium experiences a phase shift of $\Delta \phi=2 \pi n_{2} I(t) z / \lambda_{0}$ [16], which is thus also a Gaussian function (Fig. 1.2.2.1); $\lambda_{0}$ is the wavelength of the carrier wave of the pulse and $z$ is the propagation distance. 


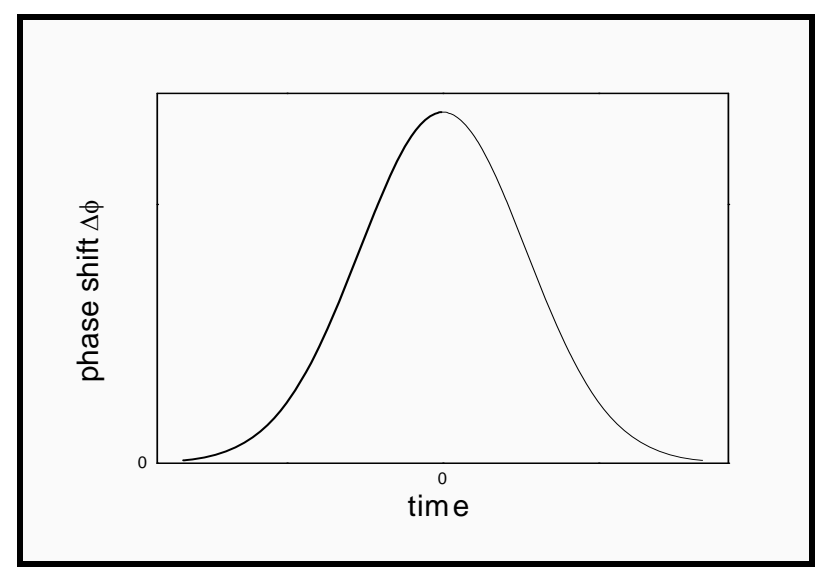

Fig. 1.2.2.1 The temporal variation of the phase shift $\Delta \phi$ follows that of the intensity $I(t)$.

This time-varying phase shift will add a time-varying frequency $\Delta \omega$ to the carrier frequency $\omega_{0}$ of the light, which is of the form $\Delta \omega(t)=-\mathrm{d}(\Delta \phi) / \mathrm{d} t=\left(4 \pi / \lambda_{0}\right) n_{2} I(t)\left(t z / \tau^{2}\right)($ Fig. 1.2.2.2). From Fig.1 2.2.2 it can be concluded that the optical pulse in its rising part will lead to a lower frequency than $\omega_{0}$ as it propagates, while a frequency $>\omega_{0}$ will emerge from its falling part, so that the travelling pulse is temporally chirped. The difference between the minimum and maximum of Fig. 1.2.2.2 gives the maximum frequency spread around the carrier frequency $\Delta \omega_{\max } / 2 \pi=2.86\left(n_{2} I_{0} / \lambda_{0}\right)\left(z / t_{p}\right)$; the Gaussian temporal width $\tau$ is connected with the full width at half maximum (FWHM) of the pulse via $t_{p}=2 \tau \sqrt{\ln 2}$.

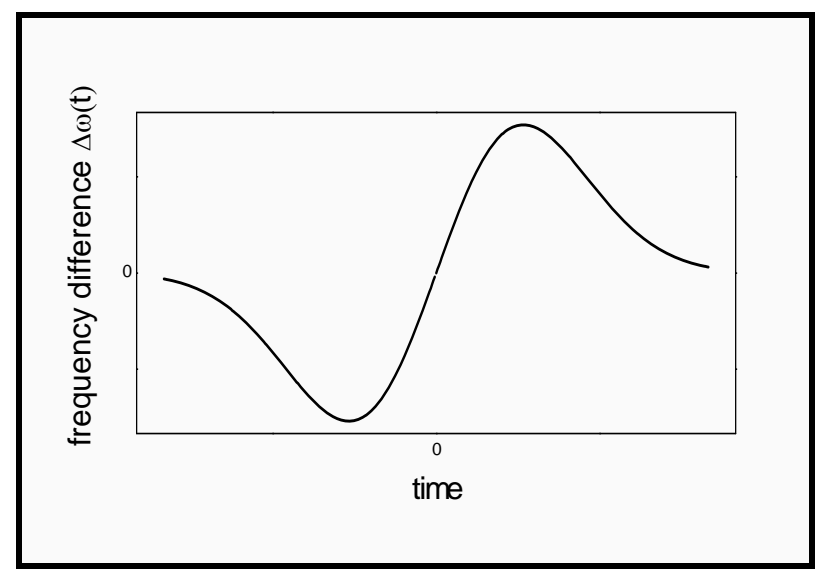

Fig. 1.2.2.2 Temporal variation of the induced instantaneous frequency difference $\Delta \omega$.

Around the peak of the pulse intensity, $\Delta \omega$ varies approximately linearly with time corresponding to a linear chirp in this region (Fig. 1.2.2.3). 


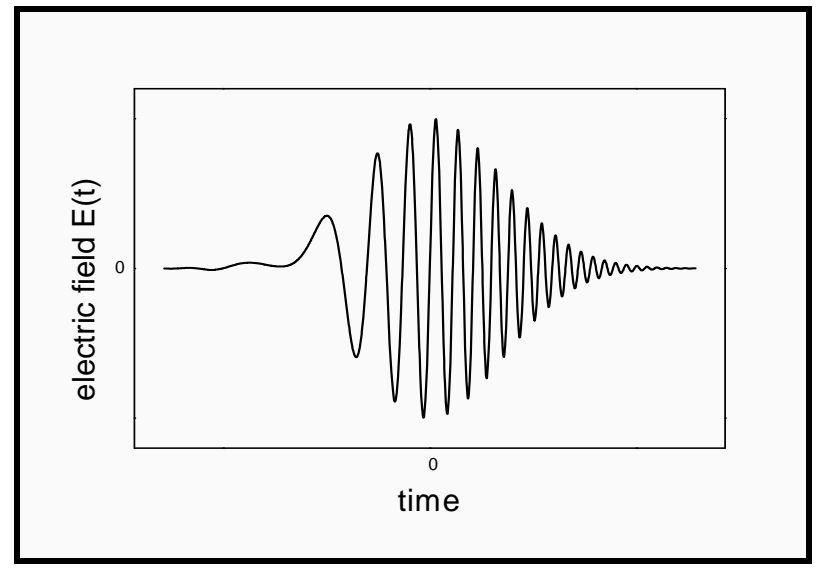

Fig. 1.2.2.3. Linearly chirped pulse

The phase shift accumulated during the propagation of the light pulse and hence the frequency difference $\Delta \omega$ is proportional to the propagation length $z$ and inversely proportional to $t_{p}$. Therefore, the spread $\Delta \omega_{\max } / 2 \pi$ increases with distance and the spectrum is continuously broadened, as long as the temporal pulse shape and the high intensity can be maintained.

A pulse can also be phase modulated in the presence of the field of a second pulse, as long as the two beams overlap in the medium in space and time; therefore, a spectral broadening of a weak pulse can be induced by means of a second strong electric field. This process is called cross phase modulation.

During propagation of an optical beam through a nonlinear and non-dispersive medium, the travelling pulse is chirped due to self-phase modulation; distortion of its temporal envelope due to dispersion will be taken into account below (sec. 1.2.3). However, even in the absence of any dispersion, a significant nonlinear distortion of the shape of the pulse envelope is possible: during propagation in the medium the part of the pulse near the peak travels with smaller velocity than its wings, because it experiences a higher index of refraction $[1,14]$ $n(t)=n_{0}+\Delta n(t)=n_{0}+n_{2} I(t)$. As a consequence the peak of the pulse is delayed compared to the wings which travel faster; this leads to a self-steepening of the falling edge of the pulse. Therefore, the intensity $I(t)$, that initially has a Gaussian temporal profile, becomes asymmetric after some propagation (Fig. 1.2.2.4). This results further in an asymmetric spectral broadening $[14,17]$. The steeper falling slope extends the spectrum further to shorter wavelengths. 


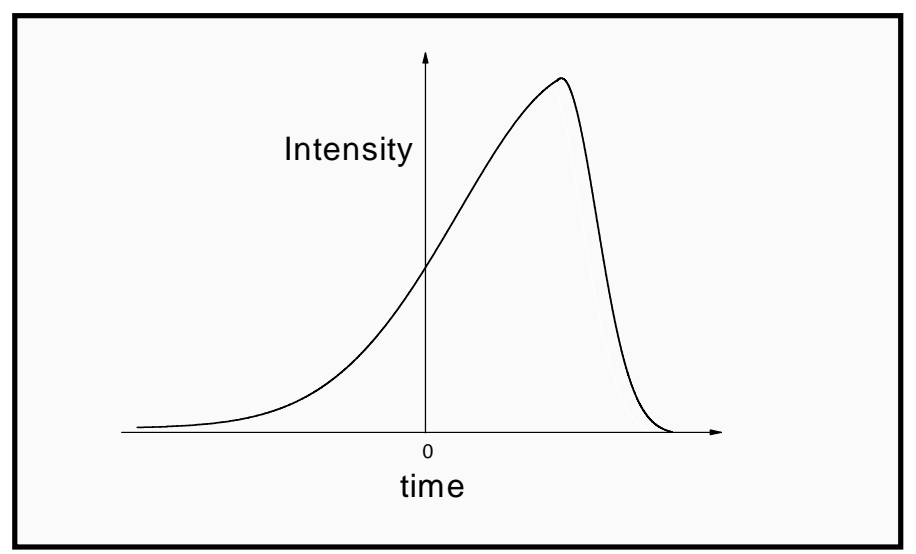

Fig.1.2.2.4 Self-steepening of a Gaussian pulse

\subsubsection{Material dispersion and devices for pulse compression}

In most practical cases the propagation medium is dispersive; this means that the phase and group velocity of different monochromatic waves that propagate through it depend on the frequency. This is an effect of the frequency dependence ("dispersion") of the linear index of refraction. It is reflected by the different phase shifts $\phi(\omega)$ that the various spectral components of a wave packet suffer upon propagation. Expanding the phase shift in Taylor series about the carrier frequency $\omega_{0}$ yields $\phi(\omega)=\phi_{0}+\phi_{1}\left(\omega-\omega_{0}\right)+(1 / 2) \phi_{2}\left(\omega-\omega_{0}\right)^{2}+$ $(1 / 6) \phi_{3}\left(\omega-\omega_{0}\right)^{3}+\ldots ; \phi_{0}$ only introduces a constant phase shift which is the same for all the frequency components. The second term $\phi_{1}=\left.(\mathrm{d} \phi / \mathrm{d} \omega)\right|_{\omega_{0}}=\left.(\mathrm{d} k / \mathrm{d} \omega)\right|_{\omega_{0}} z=T_{g}$ accounts for the time the pulse envelope needs to travel a distance $z$ and is termed group delay, while the third term $\phi_{2}=\left.\left(\mathrm{d}^{2} \phi / \mathrm{d} \omega^{2}\right)\right|_{\omega_{0}}=\left.\left(\mathrm{d}^{2} k / \mathrm{d} \omega^{2}\right)\right|_{\omega_{0}} \mathrm{z}=\mathrm{d} T_{g} /\left.\mathrm{d} \omega\right|_{\omega_{0}}$ measures the variation of this delay with the frequency $\omega$ and is called the group-delay dispersion [12]. As a consequence of $\phi_{2}$, if a pulse is spectrally broad, either due to its short temporal width or due to self-phase modulation, the different spectral parts travel at different group velocities. This group velocity dispersion distorts the temporal shape of the original pulse and this distortion becomes more significant with increasing $z$; if the dispersion is positive ("normal"), i.e. $d^{2} k / d \omega^{2}>0$, the low frequency components of the pulse travel faster than the high frequency components and the pulse thus broadens in time. This means that an initially bandwidth-limited pulse of FWHM pulse duration $t_{p}$ is lengthened to $t_{z}=t_{p} \sqrt{1+\left(z / L_{\text {disp }}\right)^{2}}$ and becomes positively chirped after a 
travelling distance $\mathrm{z}$ in a medium with group velocity dispersion (GVD) $k_{2}=\mathrm{d}^{2} k /\left.\mathrm{d} \omega^{2}\right|_{\omega_{0}}$, where $L_{\text {disp }}=t_{p}^{2} /\left(4 \ln 2 k_{2}\right)$. Values for the GVD at 800, 267 and $200 \mathrm{~nm}$ (calculated from $n(\lambda)$ given in [18]) are $k_{2}=21,82$ and $148 \mathrm{fs}^{2} / \mathrm{m}$ for 1 bar of Ar, $k_{2}=21,101$ and 302

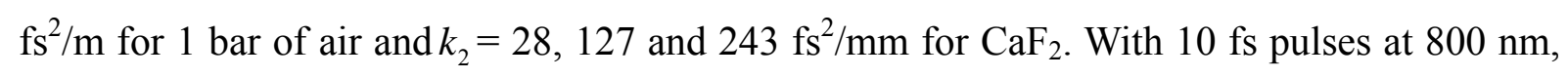
for instance, one has a dispersion length of $1.7 \mathrm{~m}$ in air and $1.3 \mathrm{~mm}$ in $\mathrm{CaF}_{2}$.

On the other hand negative ("anomalous") dispersion results in pulse compression when applied to a positively chirped pulse, because its leading part now travels slower than the trailing part. The combination of Kerr-induced self-phase modulation and negative group-velocity dispersion is used for compressing long pulses and generating ultrashort radiation of few femtoseconds [10]. If a light pulse travels through a nonlinear medium with normal or no dispersion, it becomes chirped and spectrally broadened due to self-phase modulation. If then the pulse passes through a medium or set-up with negative dispersion, it can ideally be converted into a bandwidth-limited pulse with smaller duration than the original pulse.

An arrangement with negative dispersion often used for pulse compression is the prism pair of Fig. 1.2.3.1, which consists of two prisms and a planar or flexible mirror for reflecting the beam back through the prisms [19-21]. It is the angular dispersion of the prisms combined with the frequency-dependent variation of the optical path length behind the dispersive element that gives rise to the negative group-velocity dispersion GVD of the optical pulse. The relation between angular dispersion and GVD can be derived from the ray-optical analysis performed in $[19,20]$.

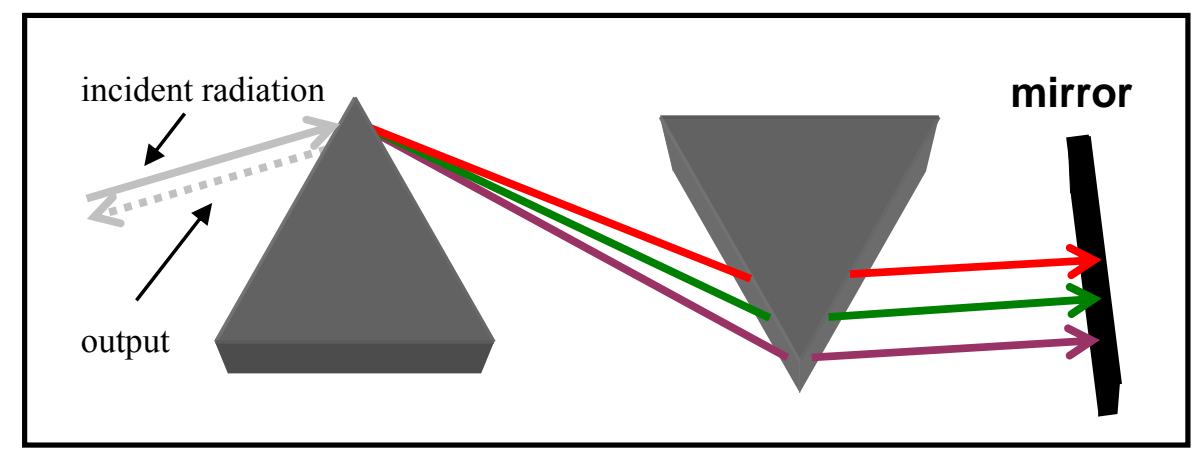

Fig. 1.2.3.1 Prism compressor

This results in a practical expression that accounts for the group-delay dispersion (GDD) $\mathrm{d}^{2}(k z) / \mathrm{d} \omega^{2}$ for the prism compressor of Fig. 1.2.3.1 as given by Diels-Rudolf [19]: 


$$
\mathrm{GDD}=\frac{\mathrm{d}^{2} \phi}{\mathrm{d} \omega^{2}}=\frac{\lambda^{3}}{2 \pi c^{2}}\left(n^{\prime \prime} l-4 L n^{\prime 2}\right)
$$

where $n^{\prime}=\mathrm{d} n / \mathrm{d} \lambda, n^{\prime \prime}=\mathrm{d}^{2} n / \mathrm{d} \lambda^{2}, l$ the length in the prism material and $L$ the distance between the two prisms. Values for $n^{\prime}$ in $\mathrm{CaF}_{2}$ at 800,267 and $200 \mathrm{~nm}$ are $-0.01,-0.3$ and $-0.8 \mu \mathrm{m}^{-1}$ respectively, while values for $n "$ are $0.03,3.8$ and $17.2 \mu \mathrm{m}^{-2}$ respectively [18]. This negative GDD (similarly also with gratings instead of prisms) can be used to compress positively chirped pulses (such as those resulting from SPM) and to compensate their temporal broadening. Moving a prism in or out of the beam, the negative GDD can be fine-tuned, profiting from the positive $n$ " of the prism material. Coarse tuning of the GDD is done by adjusting $L$.

Another device that introduces a negative GDD is the so-called chirped multilayer mirror [10] (Fig. 1.2.3.2).

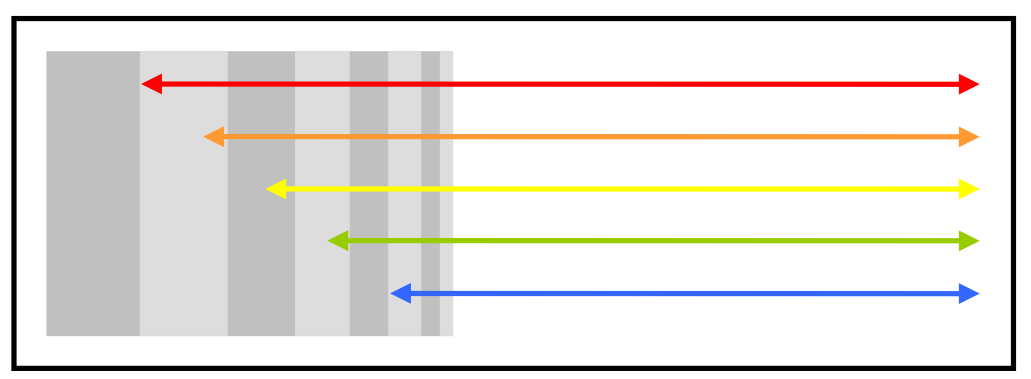

Fig. 1.2.3.2 Chirped-multilayer mirror

The principal idea of the chirped-mirror design is that the Bragg wavelength is not constant but varies within the structure, so that the different components of the incident radiation penetrate to different depths into the mirror structure before being reflected. For this purpose, appropriate modulation of the multilayer period of the dielectric mirror is required. Such a chirped mirror exhibits a wavelength-dependent group delay, which increases with longer wavelength (negative GDD), as well as dispersion of higher order favouring the production of few-cycle laser pulses. Chirped mirrors are available for $800 \mathrm{~nm}$ with GDD around $100 \mathrm{fs}^{2}$. Prism compressors, although less convenient to use, can attain much higher GDD and can also be used in the UV.

\subsubsection{Self-focusing}

If the variation of the beam profile with the transverse coordinates $x, y$ is taken into account, another important phenomenon arises. As a wave with a Gaussian transverse intensity pro- 
file $I(r)=\left(2 P / \pi w^{2}\right) \exp \left[-2 r^{2} / w^{2}\right]$ propagates through a Kerr medium, a phase-shift $\Delta \phi=$ $2 \pi n_{2} I(r) z / \lambda_{0}$ accumulates; $P$ is the power and $w$ the $e^{-2}$ radius ("waist") (Fig. 1.2.4.1).

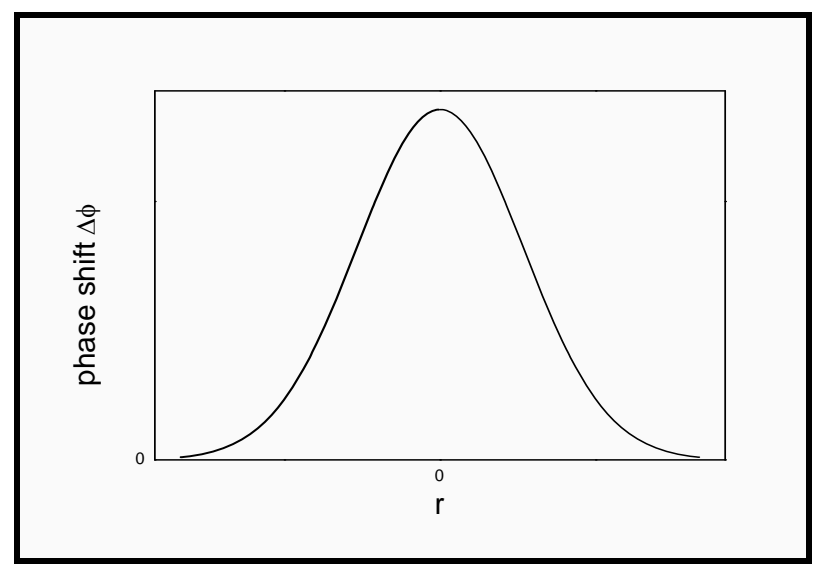

Fig. 1.2.4.1 Transverse spatial profile of the induced $\Delta \phi$

As the beam propagates in the nonlinear medium (e.g. argon in the present experiment), the centre of the beam experiences a higher index of refraction, travels more slowly and will hence be delayed compared to the wings. Therefore, the wave front (surface of constant phase), to which the optical rays are always perpendicular, is no longer planar but starts to become curved, so that the beam starts focusing in the nonlinear medium. Because this focus originates from the beam's own intensity profile, the phenomenon is called self-focusing $[14,15]$.

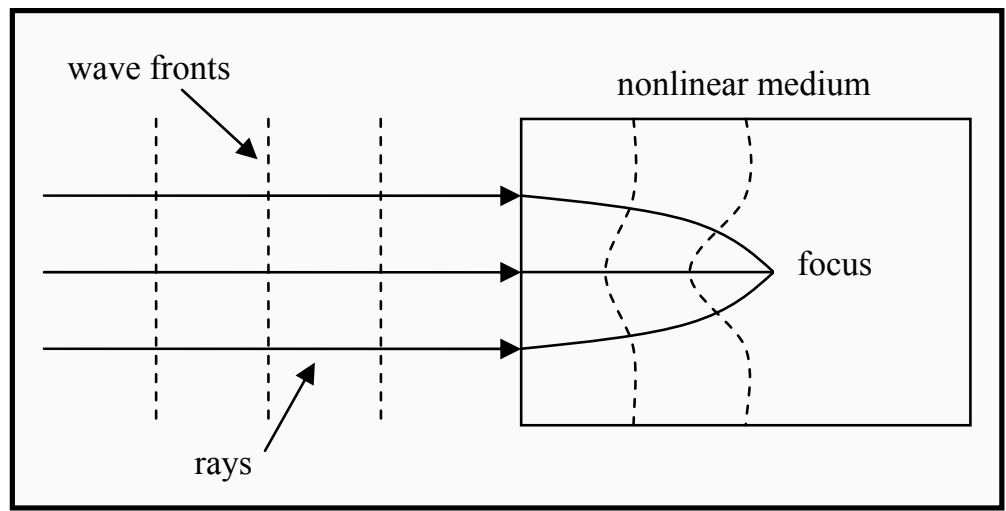

Fig. 1.2.4.2 Self-focusing

The more the beam (of high intensity) propagates in the nonlinear medium, the more it is focused so that the intensity increases further and the process is self-accelerating. On the other hand, as the beam is focused its radius becomes smaller and, as a result, diffraction increases in importance. If the laser power $P$ is equal to a critical power $P_{c r}=\lambda^{2} / 2 \pi n_{2} n_{0}$ self-focusing just balances diffraction and the initial beam waist $w$ is maintained during propagation, inde- 
pendent of its original value $[14,15]$. For argon $n_{2}=1.4 \times 10^{-19} \mathrm{~cm}^{2} / \mathrm{W}[13]$ and $P_{c r}=7.3 \mathrm{GW}$ [1]. If $P>P_{c r}$ self-focusing is stronger than diffraction and the beam would then continue focusing until it would collapse. But the high intensity that develops ionizes the gas. The resulting free electrons of density $N_{e}$ cause a negative contribution $n_{e}$ to the refractive index (since the refractive index of the plasma is lower than that of the neutral gas), which equals $n_{e}=-e^{2} N_{e} / 2 \varepsilon_{0} m_{e} \omega^{2}$. The transverse profile of the negative $n_{e}$ has thus a defocusing effect, preventing the Kerr-lens induced collapse of the beam. Over some range of laser power $P>P_{c r}$, there is a long-range propagation of the laser beam with stable diameter, which is caused by a balance of Kerr-lens focusing and plasma defocusing. Stable self-guiding has been reported over lengths that often are much larger than the diffraction lengths; they range from centimetres to kilometres, apparently depending on the initial waist (see e.g. [17, 2224]). The plasma is visible as a luminescing column along the beam axis which is known as filament $[23,24]$.

$N_{e}$ depends on the intensity of the laser $[23,25]$ namely $N_{e}(t) \propto \int_{-\infty}^{t} I^{m}\left(t^{\prime}\right) d t^{\prime}$, where $I$ is the laser intensity and $m$ is the order of ionization; it can be as large as the number of photons needed for ionization of the nonlinear medium. Therefore, the negative index contribution $n_{e}$ varies with time. The time derivative of the corresponding phase-shift generates an additional frequency shift $\Delta \omega_{e} \propto-\mathrm{d} n_{e} / \mathrm{d} t \propto I^{m}$ introduced during pulse propagation. The new frequencies produced by this physical mechanism (i.e. the temporal variation of the produced plasma) are blue shifted during both the leading and falling edge of the pulse. This mechanism hence also contributes to the spectral broadening towards the UV region. Note, however, that there is an important difference between the frequency shifts caused by the Kerr effect and those due to the plasma: whereas the latter is a function of the instantaneous intensity, the former is $\propto \mathrm{d} I / \mathrm{d} t$. This implies that with shorter pulses (of about the same maximum intensity) the contribution of self-phase modulation to the spectral broadening towards the UV gains in importance compared to that of the generated plasma.

So, also the phase shift caused by ionization gives rise to both, generation of new frequencies in a chirped pulse and spatial effects. Most importantly, the electrons of the generated plasma prevent a total spatial collapse by self-focusing of the beam and a balance between self-focusing and defocusing can be maintained over long distances (filamentation). 
Since $n_{e}$ depends on $\omega$, the plasma also causes a wavelength-dependent divergence, so that one can often observe coloured rings around the spot, with the short wavelengths outside.

For laser power $P$ much larger than $P_{\text {cr }}$ the beam breaks up into multiple filaments [23]. We indeed found [1] that under these conditions the direction of the transmitted light is unstable and attributed this to multiple filamentation (see Chapter 2).

\subsubsection{Harmonic generation}

After a wave of frequency $\omega$, polarized along the $x$ axis and propagating along the $z$ axis $\boldsymbol{E}(z, t)=\boldsymbol{x}^{0}(1 / 2) F(\omega) \exp \left[i\left(k_{\omega} z-\omega t\right)\right]+$ c.c. has passed through a nonlinear gas, the emerging radiation (besides the remaining fundamental radiation of the original frequency $\omega$ ) is composed of waves of frequency $3 \omega$ and $5 \omega$ due to the contribution to the polarization of the nonlinear terms $\chi^{(3)} \boldsymbol{E}^{3}$ and $\chi^{(5)} \boldsymbol{E}^{5}$ respectively (see section 1.2.1), depending on the conversion efficiency of the system; these terms induce in fact a polarization $\boldsymbol{P}$ which oscillates with a frequency three and five times the initial frequency of the electric field respectively, $\boldsymbol{P}_{m \omega}(z, t) \propto \boldsymbol{x}^{0} \chi^{(m)}\left\{F^{m}(\omega) \exp \left[i\left(m k_{\omega} z-m \omega t\right)\right]+c . c.\right\}, m=3,5$, which drives in turn new light waves $\boldsymbol{E}_{m \omega}(z, t) \propto \boldsymbol{x}^{0} E_{m \omega}(z) \exp \left[i\left(k_{m \omega} z-m \omega t\right)\right]+$ c.c. of frequency $3 \omega$ and $5 \omega$ (third and fifth harmonic generation), where $k_{m \omega}=n_{m \omega} m \omega / c$; the amplitude of the harmonic waves yields $E_{m \omega}(z) \propto 2 \pi N\left(k_{m \omega} / \Delta k\right)[1-\exp (i \Delta k z)] F^{m}(\omega)[26,27]$.

In order for this conversion to be efficient the driving polarization wave $\boldsymbol{P}_{m \omega}(z, t)$ and the generated optical wave $\boldsymbol{E}_{m \omega}(z, t)$ must constructively interfere and therefore have the same phase, or in other words the condition $m k_{\omega}-k_{m \omega}=\Delta k=0 \Rightarrow n(m \omega)=n(\omega)$ must be fulfilled. If the process is not phase matched, the driving polarization at $m \omega$ goes in and out of phase with the generated wave $\boldsymbol{E}_{m \omega}(z, t)$ and the conversion oscillates as $\sin (z \Delta k / 2)$ [27]. In other words, if the quantity $z_{c o h}=\pi / \Delta k$, known as coherence length, is introduced we see that the amplitude of the generated harmonic field $E_{m \omega}(z)$ grows with the propagation distance $z$ until it reaches a maximum for $z=z_{c o h}$ and then starts to drop again, the period of oscillation of the amplitude over $z$ being of the order of $z_{c o h}$. Therefore, the harmonic generation process is usually stopped after propagation of the fundamental beam of length $z_{c o h}$ (determined in relation 
to the density of the nonlinear medium appearing in $\Delta k$ ) so that the maximum harmonic yield can be obtained.

\subsection{Temporal characterization of femtosecond pulses}

The femtosecond time scale is beyond the response time of any electronic apparatus (based e.g. on a photodiode) and therefore no information about the temporal structure of subpicosecond pulses can be determined by the use of conventional electronic instruments. The simplest techniques that have been well established in the last decades for measuring the duration of femtosecond pulses are the intensity and interferometric auto- or cross-correlation [19]; when two identical pulses are correlated in the temporal characterization experimental set-up we refer to an autocorrelation scheme, while two different pulses are cross-correlated and the pulse duration of one of them can be determined if the second one is known. In autocorrelators (which are commercial at $800 \mathrm{~nm}$ ) two partial pulses from a beam splitter are superimposed with a variable delay in a nonlinear material, typically a crystal for secondharmonic generation (SHG), and the nonlinear signal is observed as a function of the delay. The resulting signal is the intensity autocorrelation function, if the two beams are not collinear; temporal interference fringes are observed if the pulses are collinear [19]. The 800-nm pulses from the laser system are measured by an intensity autocorrelator (APE) with SHG in a thin crystal, while the duration of the IR after shortening is determined by a commercial autocorrelator (Femtolasers), based on a Michelson interferometer used to produce two equal IR pulse components $E(t)$ travelling on different paths. One of them experiences a variable delay before both are recombined and focused into a very thin nonlinear crystal, where the SH is generated and then guided to the detector. The output signal is then proportional to $F(\tau) \propto \int_{-\infty}^{\infty}\left|(E(t)+E(t-\tau))^{2}\right|^{2} d t[19]$.

In this work the ultrashort UV pulses generated are temporally characterized in an intensity cross-correlation scheme, where the UV beam of intensity $I_{\mathrm{UV}}$ is collinearly combined with an IR beam (at $800 \mathrm{~nm}$ ) of known pulse duration and intensity $I_{\mathrm{IR}}$, to ionize $\mathrm{Xe}$ in the ionization region of a time-of-flight mass-spectrometer (TOFMS), by absorption of one UV photon and $m$ IR photons; a variable delay $\tau$ is introduced between the two beams and the instantaneous $X e^{+}(t)$ ion yield is proportional to $I_{\mathrm{UV}}(t) I_{\mathrm{IR}}^{m}(t-\tau)$. Due to the slow response time of the detector, however, $\mathrm{Xe}^{+}(t)$ cannot be resolved in time and the output signal $F_{\mathrm{Xe}}$ is proportional to 
the ion yield accumulated over the integration time for which the two beams temporally overlap, $F_{\mathrm{Xe}} \propto \int_{-\infty}^{\infty} I_{\mathrm{UV}}(t) I_{\mathrm{IR}}^{m}(t-\tau) \mathrm{d} t$, being a function of only the delay time $\tau$. Assuming pulses of Gaussian temporal profile, the UV pulse duration can be determined from the measured delay dependence of $F_{\mathrm{Xe}}$ and the known width of the IR pulse. 


\section{2}

\section{Ultrashort pulse generation in the I R and UV/ VUV region}

\subsection{Experimental set-up}

The commercial laser system that is used for these experiments comprises a Ti:sapphire oscillator (Spectra-Physics Tsunami), which generates short pulses, and a Ti:sapphire amplifier (Spectra-Physics Spitfire). A diode-pumped Nd:YVO ${ }_{4}$-solid-state laser (Spectra-Physics Millennia-Vs) with intracavity frequency doubling, which delivers continuous-wave light at a wavelength of $532 \mathrm{~nm}$ and power of $5 \mathrm{~W}$, is used for pumping the Ti:sapphire crystal of the oscillator and achieving the population inversion. The oscillator, which is Kerr-lens mode locked, delivers pulses at a repetition rate of $80 \mathrm{MHz}$, pulse duration of $50 \mathrm{fs}$ and energy 12.5 nJ.

The seed pulse from Tsunami enters the Ti:sapphire amplifier, which consists basically of four components, a pulse stretcher, which chirps the pulses before amplification and lengthens their duration due to positive GDD, a regenerative amplifier, which is a laser oscillator with injection mode locking and cavity dumping, a multi-pass amplifier and a pulse compressor, which has a negative GDD and recompresses the pulses; the regenerative amplifier and the multi-pass amplifier are pumped by a diode-pumped, Q-switched Nd:YLF laser (SpectraPhysics Empower) delivering a high power output of up to $30 \mathrm{~W}$ at $527 \mathrm{~nm}$ at adjustable pulse repetition rates between 1 and $10 \mathrm{kHz}$; we used $1 \mathrm{kHz}$ for all experiments. At this repetition rate, the output pulses of the Ti:sapphire laser have a pulse duration and energy of about $45 \mathrm{fs}$ and $2 \mathrm{~mJ}$ respectively.

The 800-nm pulses from the Spitfire are used for generating the supercontinuum light by self-focusing; a first step serves for shortening them (Fig. 2.1.1). The pulses ( $2 \mathrm{~mJ}$ and $\approx 45$ fs $(F W H M))$ have an initial spectral half-width of $35 \mathrm{~nm}\left(540 \mathrm{~cm}^{-1}\right)$. After attenuation of the 
energy to $\leq 1 \mathrm{~mJ}$, the beam with initial diameter of $3 w=16 \mathrm{~mm}$ is focused by a mirror of focal length $f=200 \mathrm{~cm}$ into a windowless cell of $150 \mathrm{~cm}$ length, which contains slowly flowing argon at ambient pressure (950 mbar). In some experiments a cell with $0.2-\mathrm{mm}$ thick quartzglass windows is used. The emerging pulses have a positive chirp and are spectrally and temporally broadened. The beam is recollimated by a concave mirror. To compensate the temporal broadening, the pulses after the argon cell are reflected three times by chirped mirrors (Layertec, $-120 \mathrm{fs}^{2}$ ) which introduce a negative dispersion; the resulting pulses have a duration of 10 to $11 \mathrm{fs}$, as measured by an interferometric autocorrelator (Femtolas), and a spectral half-width of $100-110 \mathrm{~nm}\left(1540-1700 \mathrm{~cm}^{-1}\right)$. There are no measurable energy losses in the cell and the pulse energy still remains $1 \mathrm{~mJ}$. A weak luminescence is observed from the focal region, from a plasma column ("filament"), approximately $10-12 \mathrm{~cm}$ long. The results are similar as in [9] (Fig. 2.1.1).

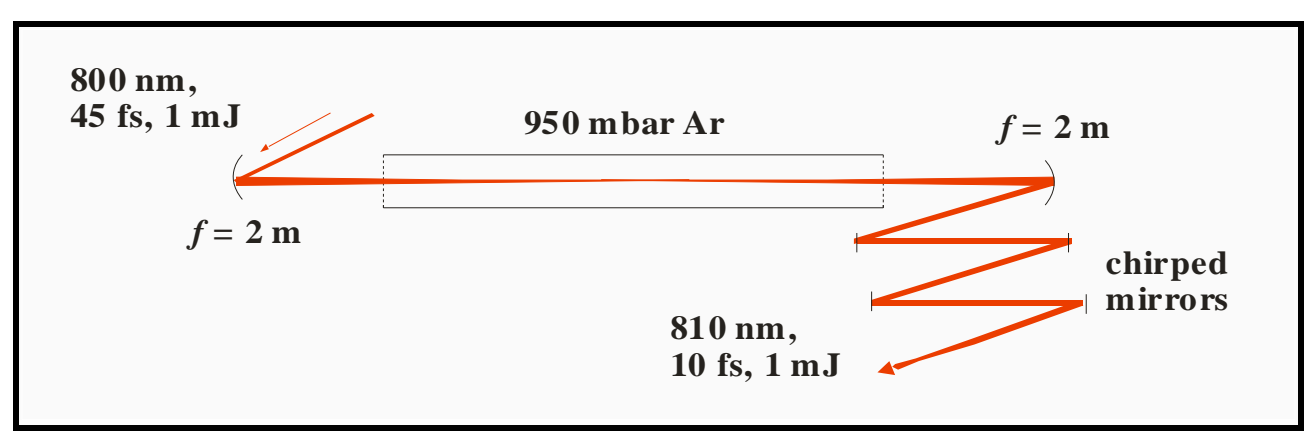

Fig. 2.1.1 Generation of few-cycle pulses in the 800-nm region.

\subsection{Supercontinuum generation}

After compression by the chirped mirrors, the beam is focused by a mirror of focal length $f=$ $100 \mathrm{~cm}$ to a second windowless cell, which also contains argon. Before the focusing mirror the beam passes through a diaphragm with a variable diameter. In the argon cell a supercontinuum is generated with properties that are discussed in the following sections. The set-up is shown in Fig. 2.2.1. The output spectra are measured by a calibrated spectrometer (Ocean Optics) with an integrating sphere at the entrance; it covers the range of $1000-200 \mathrm{~nm}$.

Fig. 2.2.2 shows the spectrum under conditions (diameter of the diaphragm $D \approx 5 \mathrm{~mm}$ and pulse energy $E=0.26 \mathrm{~mJ}$ ), for which the energy in the $300-\mathrm{nm}$ range is maximum. Dielectric mirrors centered at around 400, 290 and $267 \mathrm{~nm}$ were used to cut out the corresponding spectral regions from the beam. Fig. 2.2.2 also indicates the calculated UV power from these integrated individual spectra divided by the integrated full spectrum, as well as the measured effi- 
ciencies $\eta$ (energy divided by total pulse energy $E$ ). In the short-wavelength cut-off region, the two values differ by a factor of 2 (measured values are larger). This is probably due to the limited accuracy of the spectrometer calibration. The spectrum in Fig. 2.2.2 extends from $>1000 \mathrm{~nm}$ to $250 \mathrm{~nm}$ without any gap. Towards shorter wavelengths the $810 \mathrm{~nm}$ peak is followed by three peaks with spacing of $40 \mathrm{THz}$ and some additional smaller ones. After a shallow valley, the spectrum rises again to a maximum at around $320 \mathrm{~nm}$.

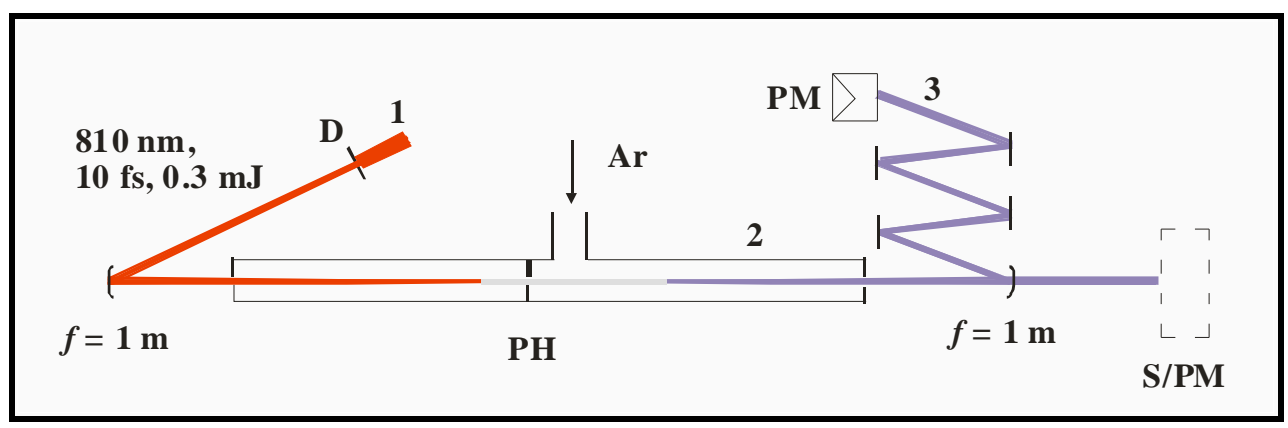

Fig.2.2.1: Scheme of the set-up, showing the second Ar cell. D: variable diaphragm; PH: optional movable pinhole; S/PM (optional): spectrometer with integrating sphere at the entrance, or power meter; PM: power meter or other devices for characterizing the UV beam; 1: Incident radiation $(810 \mathrm{~nm}, 0.7 \mathrm{~mJ}, 10 \mathrm{fs}$, produced in the first step; see Fig. 2.1.1); 2: white light emerging from the cell; 3: UV part of the radiation; the bright intermediate luminescent column indicates the filament region.

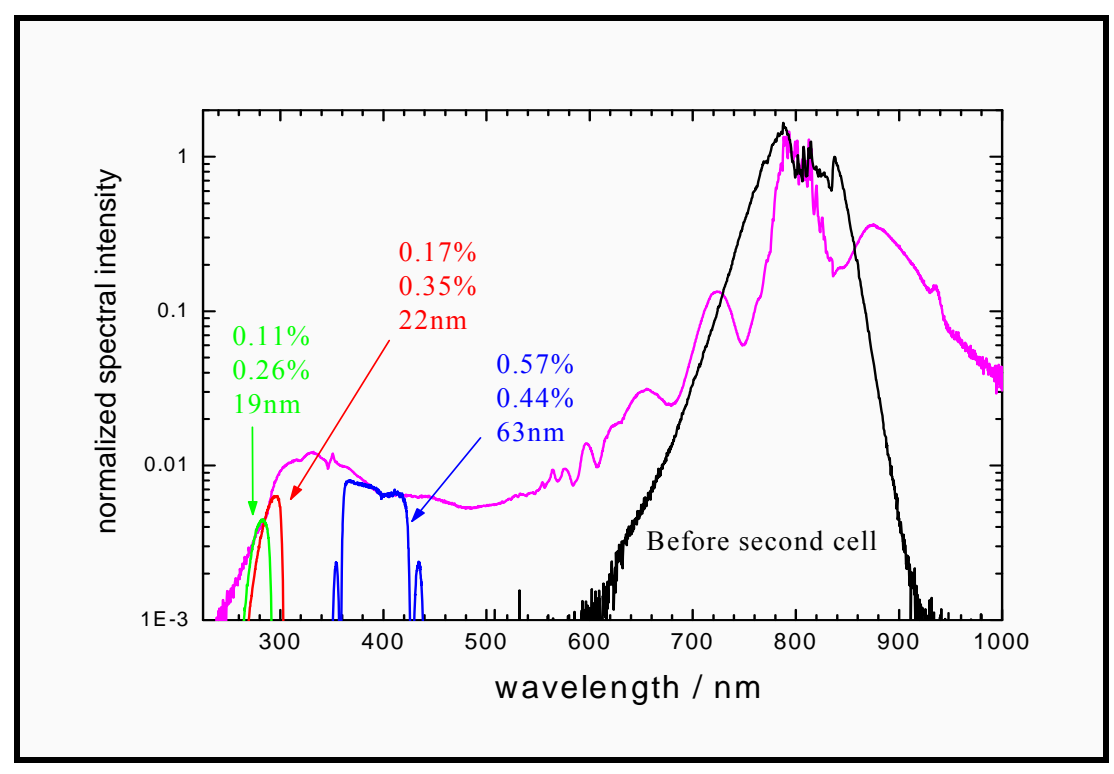

Fig. 2.2.2 Spectrum of the full supercontinuum beam after the argon cell, spectra of the 400, 290, $267 \mathrm{~nm}$ regions and spectrum of the beam before the argon cell. 
The output white-light laser pulse after propagation in argon exhibited a transverse pattern with a white central spot which contained all the spectral components, surrounded by coloured rings with larger wavelengths extending in the outer rings (photograph in section 2.3.7).

\subsection{Characterization of the suppercontinuum radiation}

\subsubsection{Variation of the input iris}

The pulse energy or power is expected to affect the spectrum substantially. The energy is varied by changing the diameter of the input diaphragm $D$. Indeed, considerable changes were found in the extension of the supercontinuum towards shorter wavelengths. Spectra were measured as a function of the diameter $D$, for $D<5.5 \mathrm{~mm}$, as well as for $D=6.3 \mathrm{~mm}$ (Fig. 2.3.1.1). This figure shows that the spectrum steadily spreads towards the UV, as the pulse energy $E$ increases up to a certain limit; the UV fraction below $300 \mathrm{~nm}$ is maximum at $E=$ $0.26-0.3 \mathrm{~mJ}$, while for $D=6.3 \mathrm{~mm}(E=0.34 \mathrm{~mJ})$ the extension of the spectrum towards UV drops again.

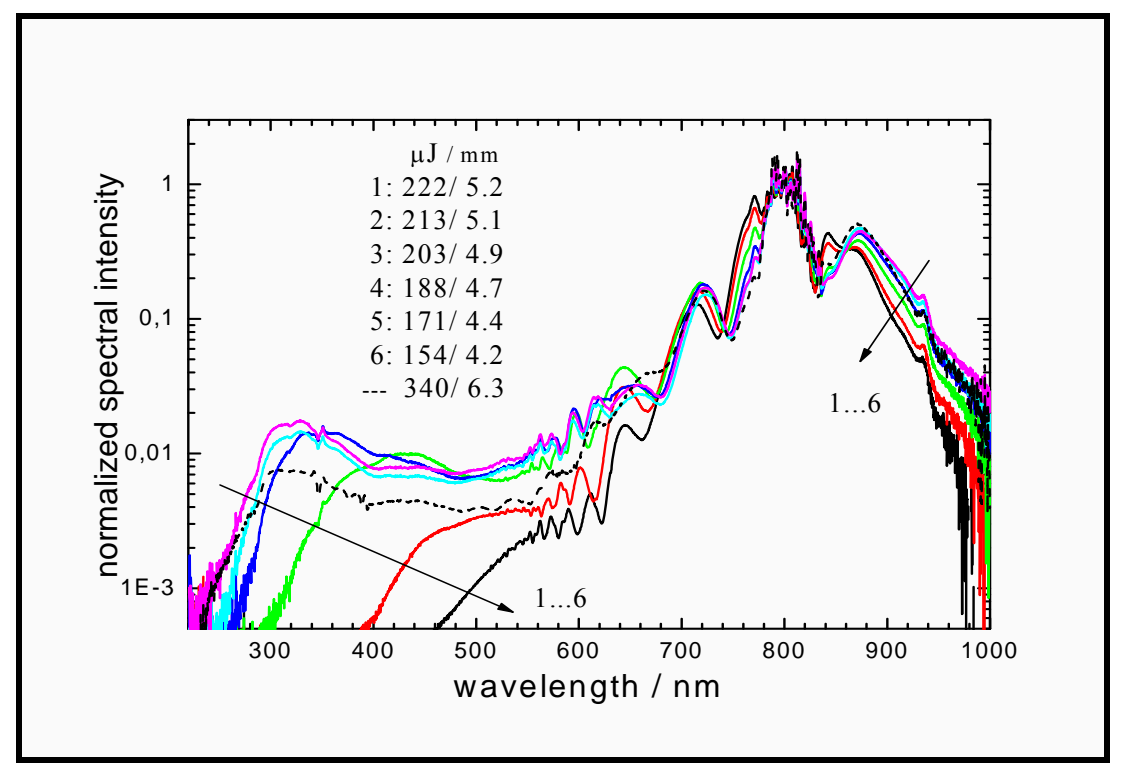

Fig. 2.3.1.1: Spectra of the output supercontinuum beam versus diameter of the input diaphragm. Spectra numbers 1 to 6 refer to solid lines, whereas the dotted line represents the spectrum generated by the full $0.3-\mathrm{mJ}$ input beam.

If the full beam of $\approx 0.7 \mathrm{~mJ}$ (totally open diaphragm) is focused into the argon cell, a very bright luminescing column of $5-7 \mathrm{~cm}$ length is observed at around $10 \mathrm{~cm}$ before the geometrical focus. The radiation emitted in forward direction has a very poor pointing stability, giving rise to a dancing white spot on a paper. This phenomenon is probably caused by multiple 
filamentation. When reducing the diameter of the diaphragm to $D \approx 5 \mathrm{~mm}$ and the corresponding transmitted energy to $E=0.3 \mathrm{~mJ}$, the instability suddenly stops. For pulse energy $E \leq 0.3$ $\mathrm{mJ}$ the luminescing column was less bright, longer and thinner (estimated: $0.1 \mathrm{~mm}$ ) and had a length of $\approx 15 \mathrm{~cm}$ with its center being at $\approx 3-4 \mathrm{~cm}$ before the geometrical focus (it extended from $94 \mathrm{~cm}$ to $109 \mathrm{~cm}$ away from the focusing mirror).

Fig. 2.3.1.2 shows the UV energy measured after reflection by four 270-nm mirrors as a function of the diameter $D$ of the input iris, corresponding to the input pulse energies $E$. The produced UV energy grows up to $D \approx 6 \mathrm{~mm}$ and then it starts dropping again for larger diameters, even though the pulse energy is higher. For $D>5 \mathrm{~mm}$ the output beam was unstable most probably due to multiple filamentation and production of UV energy dropped considerably under these conditions (Fig. 2.3.1.2). This measurement thus confirms the results of Fig. 2.3.1.1. (The $D$ value for the instability threshold differs slightly. But this value critically depends on the alignment of the input diaphragm.) Fig. 2.3.1.2 very clearly shows the nonmonotonic dependence of the UV energy on the incident energy.

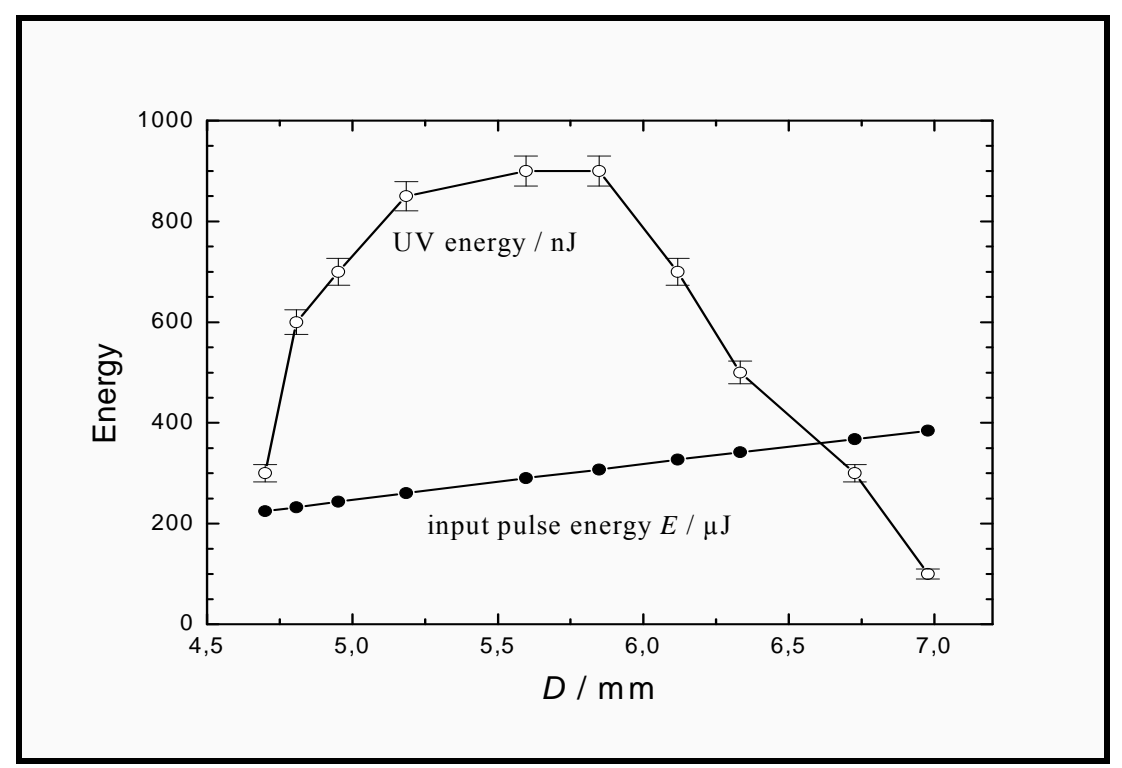

Fig. 2.3.1.2: Produced UV energy and input pulse energy of the output beam as a function of the diameter $D$ of the input diaphragm.

\subsubsection{Transverse beam profile in the filament region}

Pinholes of different diameters $d_{p}$ are inserted in the second Ar cell some centimetres after the centre of the luminescing region, at a distance of $104 \mathrm{~cm}$ from the focusing mirror. The $d_{\mathrm{p}^{-}}$ dependent transmission of the beam allows the determination of the beam profile in the self- 
focus. Fig. 2.3.2.1 shows the transmitted energy divided by the total energy without pinhole (divided by 0.86 ) versus the pinhole radius $\alpha$; the value 0.86 is just the fraction of energy contained in the central spot of an Airy diffraction pattern caused by the input diaphragm, without the outer ring. The central spot is then simulated by a Gaussian profile in the following way: the beam waist $w_{0}$ is fitted to the measured pulse energies $P(\alpha)$ (after the corresponding pinhole of radius $\alpha$ ) and $P_{0}$ (without pinhole), using $P(\alpha) / P_{0}=1-\exp \left[-2 \alpha^{2} / w_{0}^{2}\right]$ [16]. The average value of the fit parameter is $w_{0}=158 \mu \mathrm{m}$. Now the factor of 0.86 chosen above can also be further justified. The transmissions with diameters $d_{p}=3 w_{0}$ and $d_{p}=w_{0}$ were $85 \%$ and $28 \%$ respectively. Both are just $86 \%$ of the numbers calculated for a Gaussian profile. Obviously this is just due to the $14 \%$ in the outer ring of the Airy pattern, caused by diffraction at the input iris.

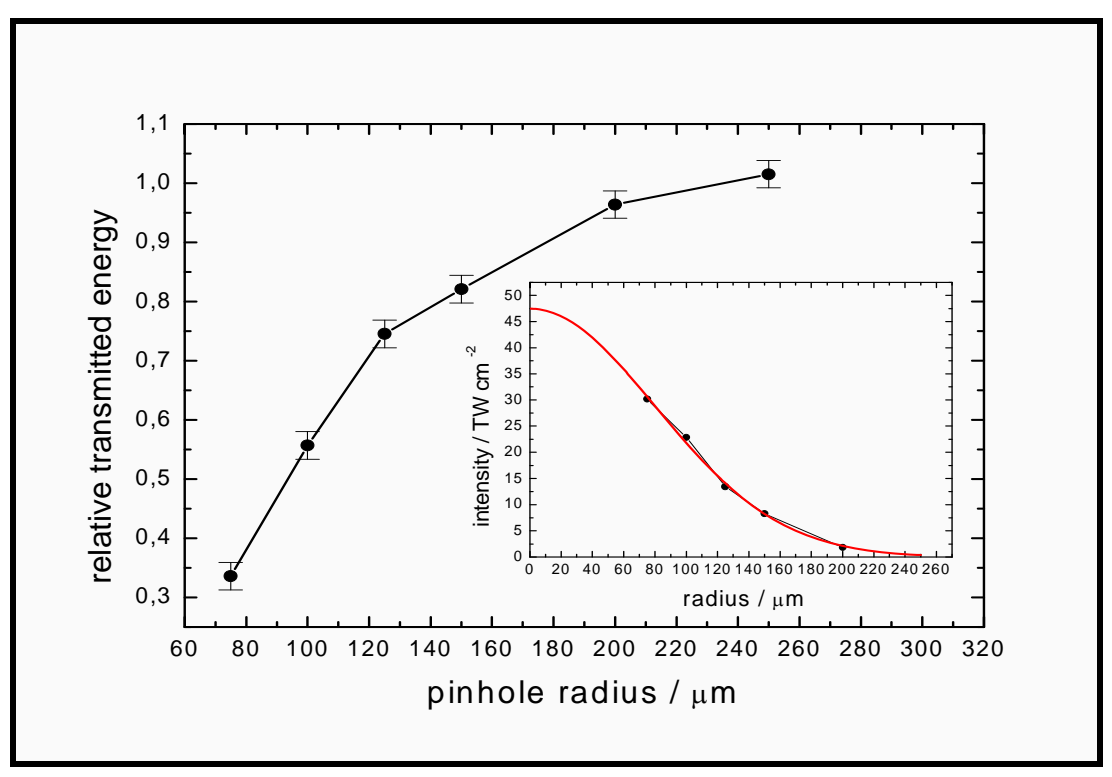

Fig. 2.3.2.1 Relative transmitted energy $P / P_{0}$ versus pinhole radius.

Twice the Rayleigh length is $2 z_{R} \approx 20 \mathrm{~cm}$, as calculated from $w_{0}\left(z_{R}=\pi w_{0}^{2} / \lambda\right)$, which is more than the observed filament length $(\approx 15 \mathrm{~cm})$. In experiments with longer pulses filaments often extended over more than the diffraction length $\left(2 z_{R}\right)$, whereas in experiments with even shorter pulses $(\approx 6 \mathrm{fs})$ we found an even shorter filament $(\approx 7 \mathrm{~cm})[28]$.

The inset of Fig. 2.3.2.1 shows the transverse intensity profile of the beam near the centre of the luminescing region (calculated from the corresponding transmitted energies and fitted by a Gaussian curve (red line)). The extrapolated intensity on the axis is $47 \mathrm{TW} / \mathrm{cm}^{2}$. This is 
similar to values reported previously, where it was also shown that ionization and the accompanying defocusing "clamps" the intensity to a maximum value (e.g. [22, 29]). The Gaussian fit gives for the waist a value of $w_{0}=160 \mu \mathrm{m}$, similar to the value calculated for Gaussian optics with an initial beam diameter (after the input diaphragm) of $D=3 \mathrm{w}=5.3 \mathrm{~mm}$ and a focal length of $f=1 \mathrm{~m}$ according to $w_{0}=\lambda f / \pi w$ where $\lambda$ is the central wavelength $(800 \mathrm{~nm})$. One can conclude that Gaussian optics fits well to the profile of the central spot and the propagation (focal length and length of high-intensity zone).

Evaluating the UV transmissions of the pinholes in the same way results in $w_{0}=140 \mu \mathrm{m}$ for the UV beam, i.e. only $13 \%$ less than the waist of the total radiation. Fig. 2.3.2.3 shows the transmitted UV energy divided by the total energy without pinhole, as a function of the pinhole radius $\alpha$; From this figure one can see that the UV conversion efficiency increases up to $\alpha=150 \mu \mathrm{m}$ and then stays constant within the estimated error limit. (Note that the error bars in Fig. 2.3.2.3 indicate the scattering with a given pinhole. Varying $\alpha$ by exchanging the pinhole introduces additional uncertainties via the alignment.)

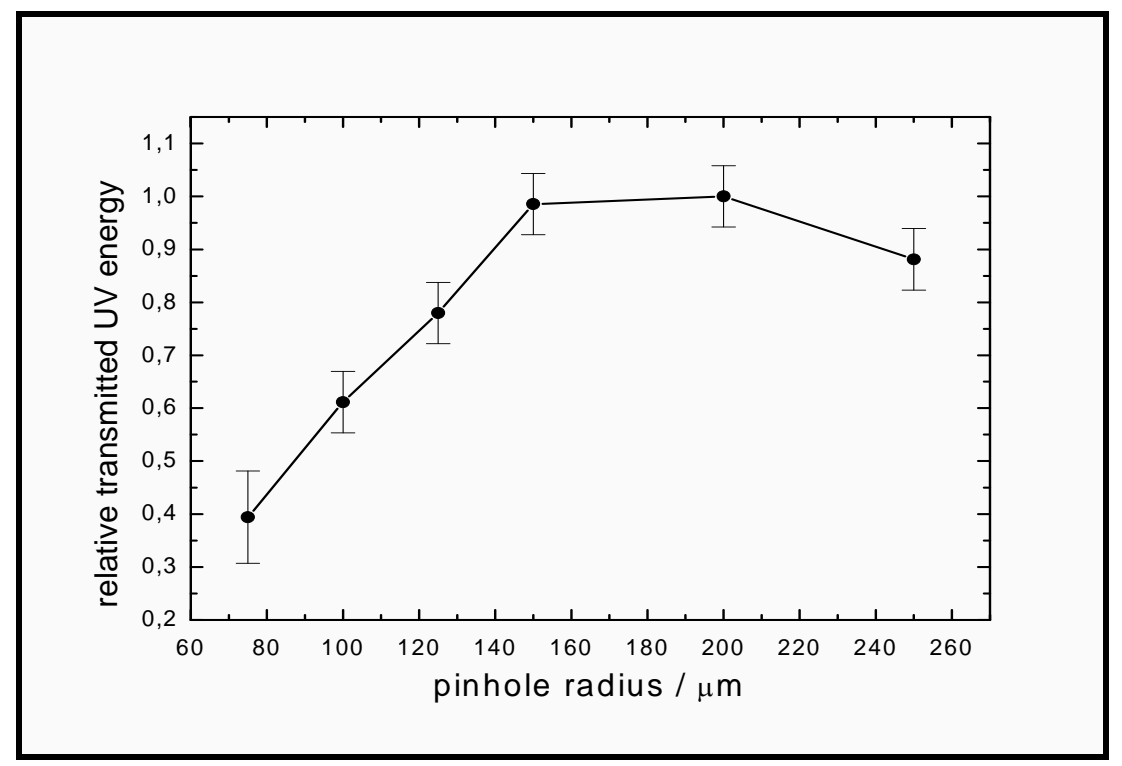

Fig. 2.3.2.3 UV conversion efficiency versus pinhole radius.

\subsubsection{Influence of the interaction length}

When the pinhole with $d_{p}=150 \mu \mathrm{m}$ diameter is inserted in the argon cell the luminescence behind it is largely suppressed (with the $200-\mu \mathrm{m}$ pinhole it was still visible, see section 2.3.2), so that self-guiding, SPM and UV production obviously stops after the hole and the beam be- 
gins to diverge. Change of the position of the pinhole in the cell thus results in different highintensity interaction lengths in the nonlinear medium. For 15 different distances of the pinhole from the focusing mirror the total pulse energy as well as the UV energy (around $270 \mathrm{~nm}$ ) transmitted through the pinhole was measured. Fig. 2.3.3.1 a shows the results, divided by total output energy without pinhole.
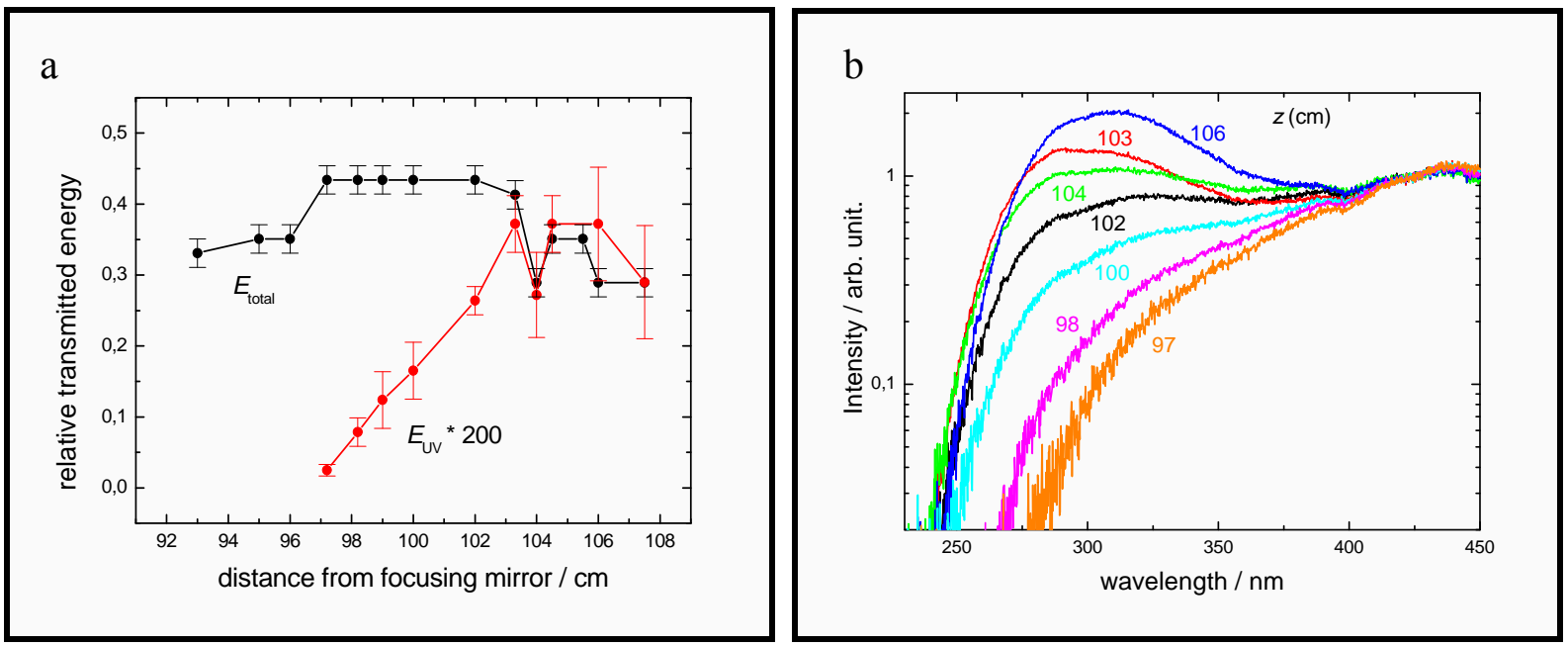

Fig. 2.3.3.1 (a) relative total and UV energy transmitted through the movable pinhole as a function of the interaction length in the argon cell. The UV energy is multiplied by 200 , so that comparison with the total energy is possible; (b) UV spectra measured after the argon cell for different propagation distances $z$.

The total transmitted energy increases from $93 \mathrm{~cm}$ to $97 \mathrm{~cm}$; from $97 \mathrm{~cm}$ to $103 \mathrm{~cm}$ the transmittance is constant and starts dropping again from $103 \mathrm{~cm}$ to $106 \mathrm{~cm}$. The flat transmission at $97-103 \mathrm{~cm}$ indicates the length of the waist. UV generation starts at the beginning of the focal region (i.e. $97 \mathrm{~cm}$ ) and increases up to the end of the visible filament. SPM, which requires high intensities, terminates after the fundamental pulse has passed through the 150 $\mu \mathrm{m}$ pinhole, since only a small part of the initial energy can be transmitted (28\%). Therefore the UV radiation is produced during propagation of the pulse before the pinhole. It increases for larger propagation distances $z$ after the focusing mirror in the nonlinear medium. The same conclusion can also be derived from Fig. 2.3.3.1b, where UV spectra, measured after the pinhole (for 7 different positions of the pinhole), are shown (they are normalized at $450 \mathrm{~nm}$ ). The spectral shapes show that the UV part steadily grows with the interaction length.

\subsubsection{Effect of the pulse duration}

The effect of the pulse duration of the initial 810-nm pulses on the generation of supercontinuum radiation was also studied. The fundamental radiation had durations of 45, 17, 10 and 
6 fs. The 45 -fs pulses came directly from the output of the laser system, while the 10 -fs pulses were produced as described in section 2.1; the 17-fs pulses were produced in the same way in the first cell, which now contained a mixture of 250 mbar Ar and 700 mbar He (see also [3]); finally the 6-fs pulses are from the laser system LWS01 in Garching, described in [30]. The energies were $1,0.4,0.3$ and $0.2 \mathrm{~mJ}$ respectively and the pulses were focused $(f=1 \mathrm{~m})$ into $\mathrm{Ar}$ at ambient pressure (950 mbar). The results for the 10-fs pulses were reported in the preceding sec. 2.3.1-3.

Fig. 2.3.4.1 a-c shows the input as well as the supercontinuum spectra generated by the 45, 17- and 6-fs pulses; for the case of the 10 fs, see Fig. 2.2.2. Fig. 2.3.4.2d shows all output spectra generated from the four initial pulse durations for comparison. From the spectra it is obvious that the UV energy as well as the spread of the supercontinuum spectrum towards shorter wavelengths increases with the shorter initial pulses. On the other hand the spectral half-widths of the emerging spectra seem to narrow as we go from the 45-fs down to the 6 -fs pulses. More specifically, the FWHM of the former is broadened from 30 to $100 \mathrm{~nm}$, while that of the 10-fs pulses is narrowed from 100 to $50 \mathrm{~nm}$; this effect is even more pronounced for the 6-fs pulses as their half-width decreases from $250 \mathrm{~nm}$ to $70 \mathrm{~nm}$. There is no such tendency, however, for the 17-fs pulses as the data show a broadening of both the FWHM and the pedestal of the supercontinuum spectrum after the argon cell.
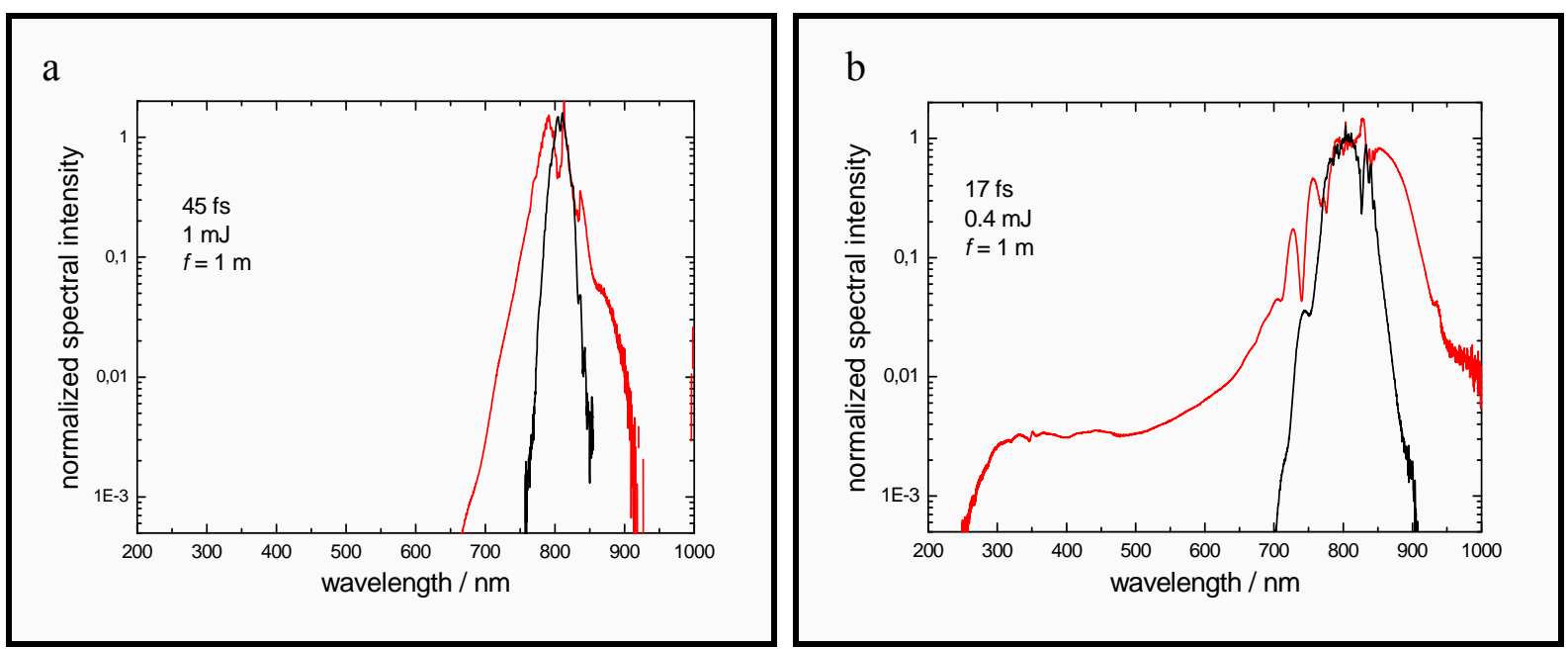

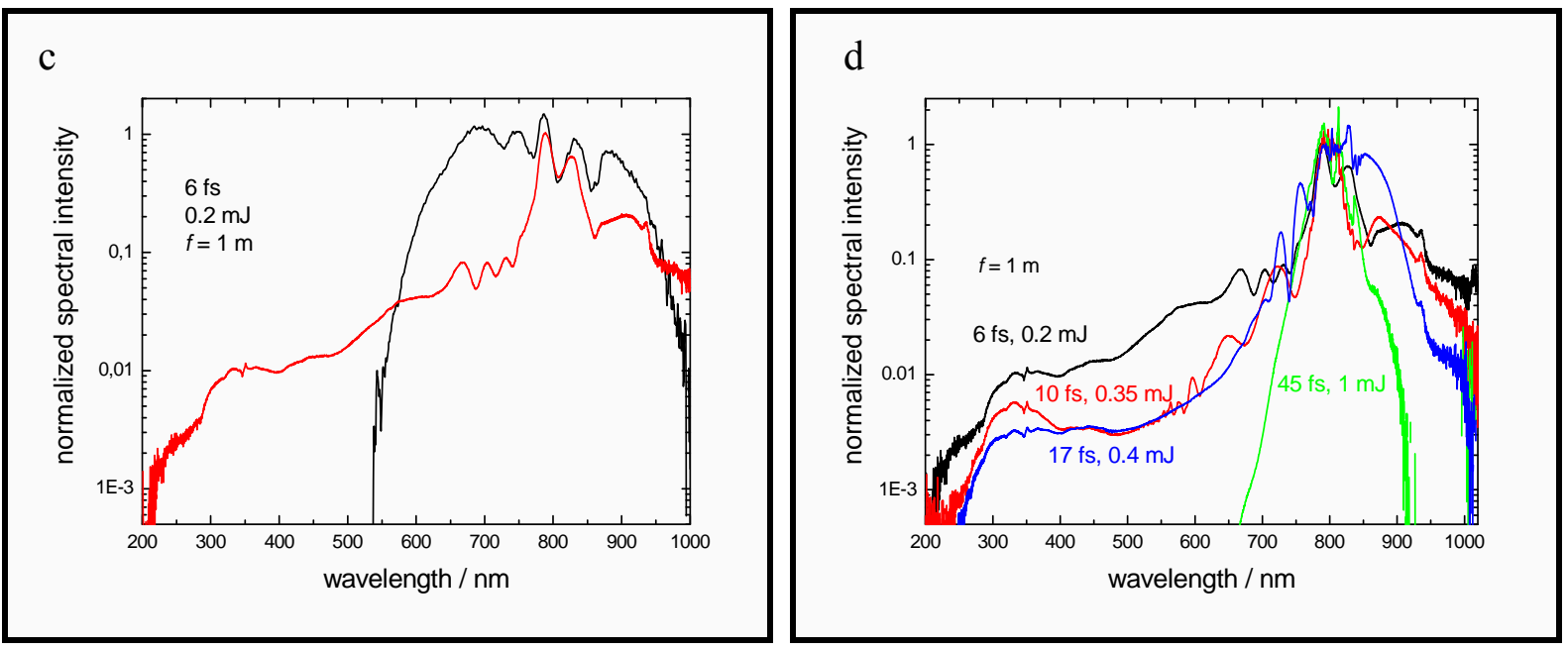

Fig. 2.3.4.1 The input and supercontinuum spectra generated by the (a) 45-, (b) 17-, and (c) 6-fs pulses; (d) effect of pulse duration in the supercontinuum generation.

\subsubsection{Effect of the focal length}

The influence of focusing before the argon cell where supercontinuum generation occurs was investigated by varying the focal length between 1 and $2 \mathrm{~m}$. Fig. 2.3.5.1a, b shows the supercontinuum spectra emerging from the 10- and 45-fs initial pulses after focusing with 1- or 1.5$\mathrm{m}$ and 1- or 2-m focal lengths respectively. The energy of the pulses was around $0.3 \mathrm{~mJ}$ and 1 $\mathrm{mJ}$ respectively. Clearly there is a strong influence of the focal length with the 45 -fs pulses, while the effect is practically absent with the shorter pulses; with $f=2 \mathrm{~m}$ the former reaches a spectral cut-off of $250 \mathrm{~nm}$, which is equal to that reached by the 10-fs pulses when focused with $f=1 \mathrm{~m}$. The length of the filament increases is in this case (compared to the filament length obtained with tighter focusing), which means that the interaction length for SPM is now longer and therefore the conversion to shorter wavelengths more efficient.
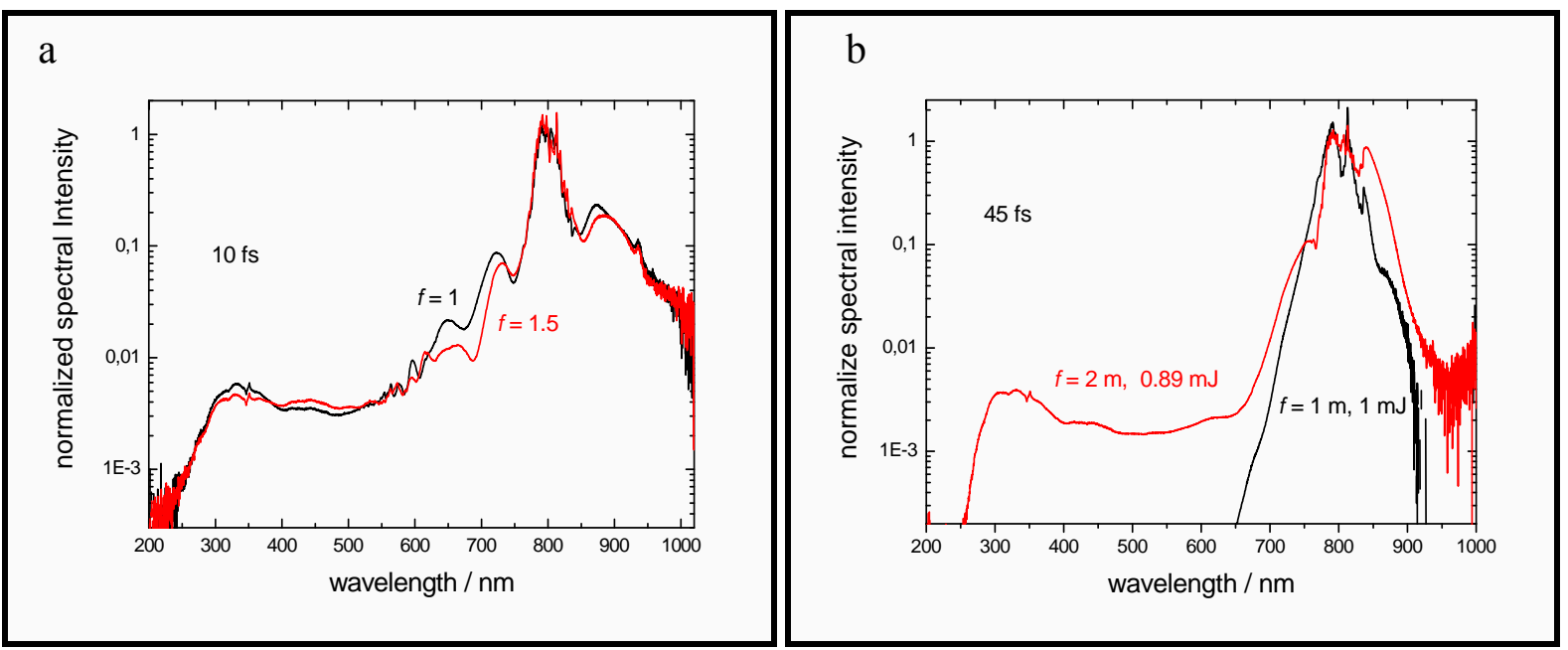
Fig.2.3.5.1 Effect of focal length in the supercontinuum spectra (a) with $10 \mathrm{fs}$ (conditions the same as in Fig. 2.2.2) and $f=1 \mathrm{~m}$ or $1.5 \mathrm{~m}$ and (b) with $45 \mathrm{fs}$ and $f=1 \mathrm{~m}$ or $2 \mathrm{~m}$.

\subsubsection{Variation of the initial chirp}

In all the experiments reported above the input 810-nm pulses generating the supercontinuum radiation were near the transform limit and therefore were not chirped. If their initial temporal chirp (and consequently the pulse duration) is varied the output supercontinuum spectrum is influenced. For the 45 -fs pulses the chirp was changed by varying the distance of the grating from the retroreflector in the compressor of the laser. The rest of the conditions $(E=0.7 \mathrm{~mJ}$ and $f=2 \mathrm{~m}$ ) were kept constant to the optimum value for UV generation when the initial chirp is zero (section 2.3.5). Fig. 2.3.6.1 shows the spectral cut-off as well as the FWHM of the supercontinuum spectrum as a function of the input pulse duration, which is an indirect measure of the chirp.

From Fig. 2.3.6.1 we observe that the maximum spread of the output spectrum towards the short wavelength region is achieved when the input pulses are transform-limited, whereas the broadest half-width is achieved when the input pulses are positively chirped and have a duration of approximately $85 \mathrm{fs}$. In this case the supercontinuum FWHM is $155 \mathrm{~nm}$ and would support a duration of $6 \mathrm{fs}$ in the transform limit (assuming Gaussian pulses); when, on the other hand, the pulses are negatively chirped, having an initial duration around $60 \mathrm{fs}\left(\tau-\tau_{0}=15\right.$ fs), the half-width of the spectrum is the narrowest, $17 \mathrm{~nm}$, (i.e. narrower than the initial FWHM) supporting a duration of approximately $55 \mathrm{fs}$. That is, under these conditions the resulting pulses are practically transform-limited.

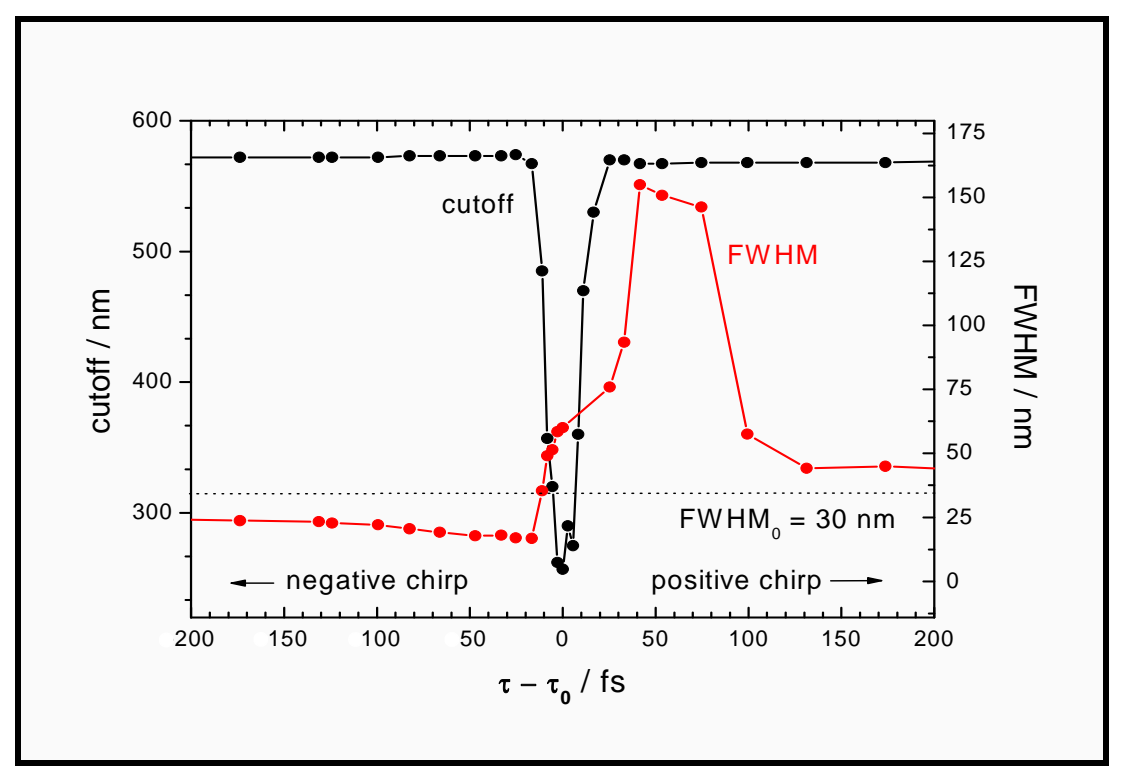


Fig. 2.3.6.1 The cut-off (black circles) and the half-width (red circles) of the supercontinuum spectrum generated by the 45 -fs input pulses as a function of their initial chirp. The chirp of the pulses was varied directly in the compressor of the laser system. Their energy was $0.7 \mathrm{~mJ}$ and the focal length $2 \mathrm{~m}$.

For the 10-fs pulses the influence of the initial chirp is also checked by recording the spectra of the output beam for different initial chirps, this time varied by changing the number of reflections from the chirped mirrors and by introducing a window before the 810 -nm pulses enter the second argon cell of Fig. 2.1.1. Four different cases were investigated: three reflections by $-120 \mathrm{fs}^{2}$ chirped mirrors, two reflections by $-120 \mathrm{fs}^{2}$ chirped mirrors, three reflections by $-120 \mathrm{fs}^{2}$ chirped mirrors and one reflection by a $-70 \mathrm{fs}^{2}$ chirped mirror and finally four reflections by $-120 \mathrm{fs}^{2}$ chirped mirrors and an inserted 2-mm thick $\mathrm{CaF}_{2}$ window between the mirrors and the nonlinear cell. Such a window introduces a positive group delay dispersion of $70 \mathrm{fs}^{2}$. Fig. 2.3.6.2 shows the corresponding full spectra (labelled with the number of reflections, type of mirror and window and pulse duration as measured before the cell).

From this figure it can be seen that the pulse with the shortest duration was the most efficient in the UV generation. But the duration seems not to be the only criterion, since the pulse with duration of $29 \mathrm{fs}$ seems to have the same efficiency in the UV as the 13-fs pulses. Furthermore, the initial chirp affects the spectral modulations. For instance, the spectrum of the 13 -fs pulse that is reflected by three $-120 \mathrm{fs}^{2}$ mirrors and one $-70 \mathrm{fs}^{2}$ mirror exhibits significantly more peaks than the other spectra.

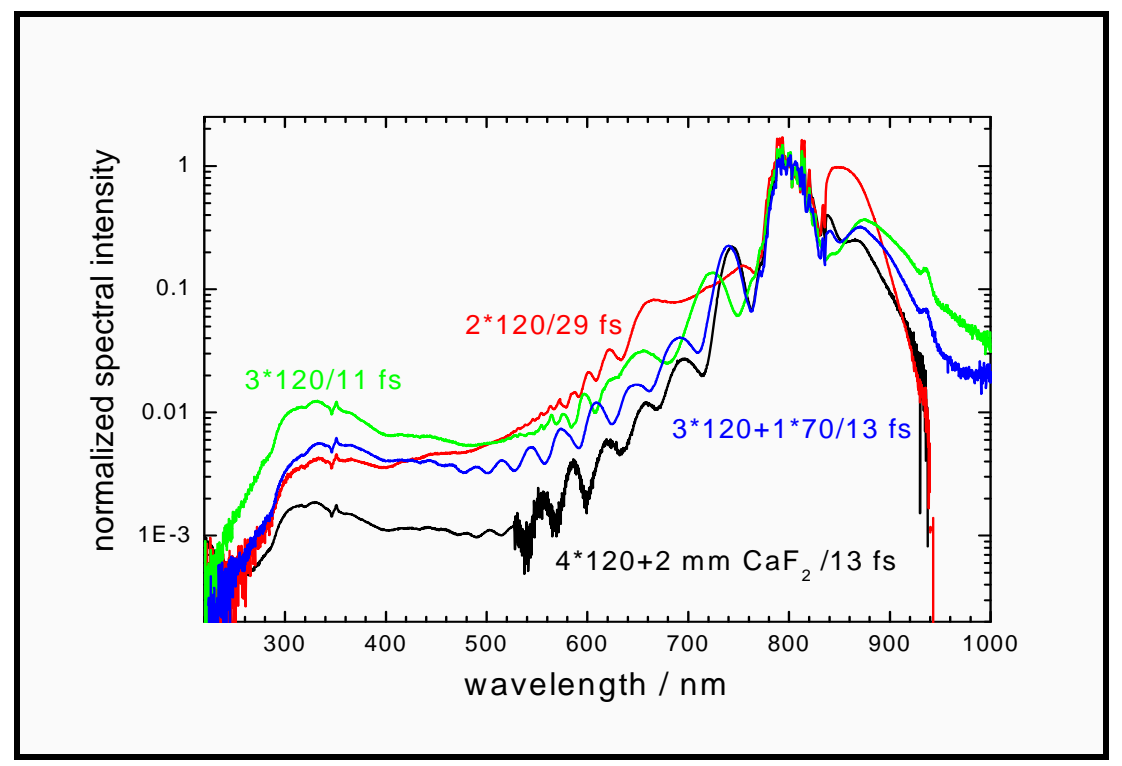

Fig. 2.3.6.2 Spectra measured after the argon cell for 4 different initial chirps. 


\subsubsection{Spatial variation of the supercontinuum spectrum}

Fig. 2.3.7.1 shows the spectra recorded when an iris of variable diameter is placed after the argon cell, before the spectrometer, in the centre of the output beam. The input pulse duration and energy were $10 \mathrm{fs}$ and $0.26 \mathrm{~mJ}$ respectively. From this figure it can be seen that reducing the diameter of the iris results only in reducing the red part of the spectrum, while the region near the UV remains practically unchanged. Obviously, the UV part of the supercontinuum spectrum is mainly generated on the axis. Fig. 2.3.7.1 shows additionally two photographs of the beam profile, one after dispersion by $\mathrm{CaF}_{2}$ prism, indicating that the centre of the beam contains all wavelengths and extends farthest into the UV range. (The red ring that surrounds the central spot in the non-dispersed photograph contains about $14 \%$ of the total energy and is probably the second maximum of the Airy pattern caused by the input iris.)
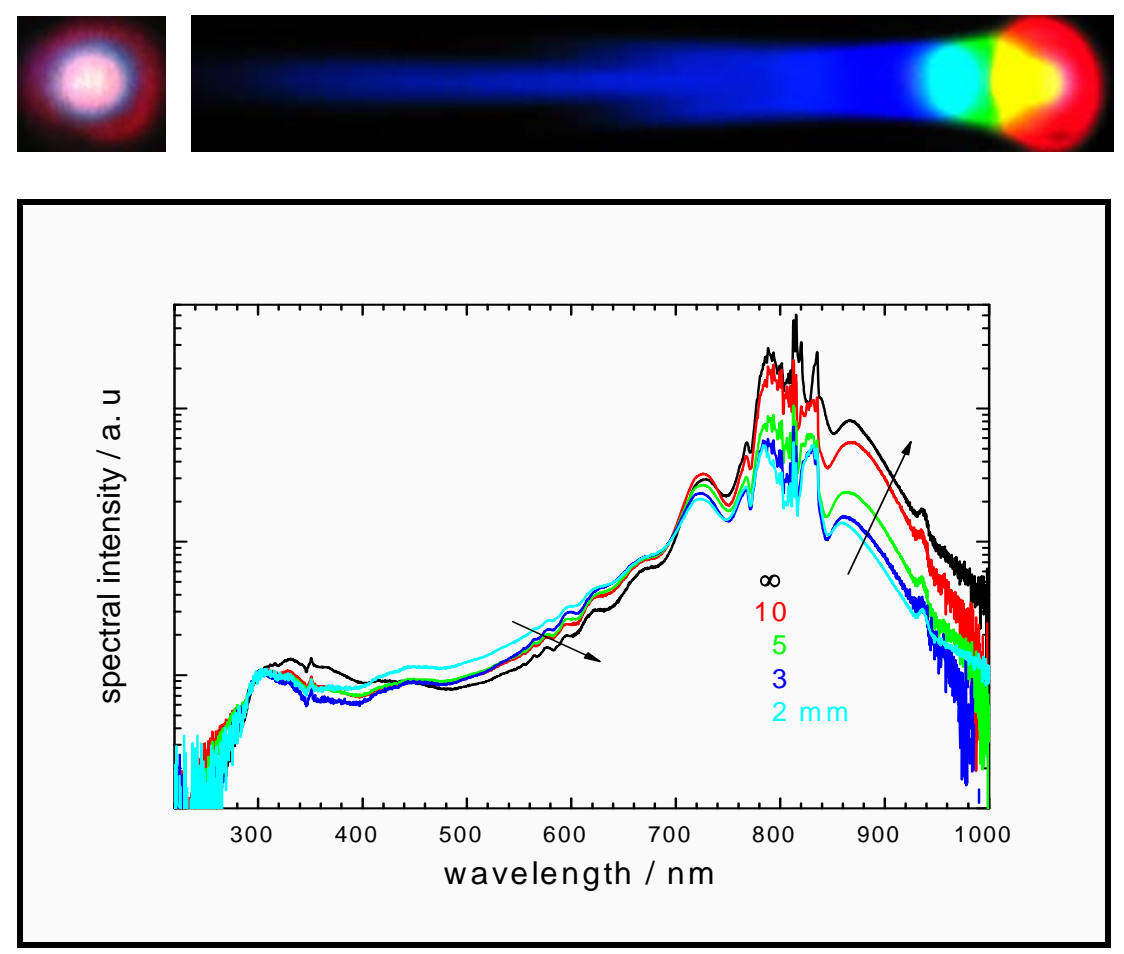

Fig. 2.3.7.1 Spatial variation of the supercontinuum spectrum generated by the $10-\mathrm{fs}, 810-\mathrm{nm}$ pulses (the spectra are normalized in the 300-nm region to 1 for comparison); an aperture of variable diameter was placed before the spectrometer that recorded the spectra. The photograph shows a spectrum made by dispersion of the collimated beam by a $\mathrm{CaF}_{2}$ prism; the shortest wavelengths extend to the $\mathrm{UV}$ region.

For the 45-fs pulses on the other hand, blue and UV rings appear to surround the central spot of the beam (not shown); this effect is in agreement with previous observations of "conical emission" in filamentation, where the red part is generated on the axis and the beam is sur- 
rounded by coloured rings (e.g. [23, 31, 32]). If there is any conical emission in the case of the 10 -fs pulses the cone angles must be too small to be detected.

\subsubsection{Argon partial pressure}

Mixtures of Ar and He were tried in the second cell, with the 10 -fs pulses $(E=0.3 \mathrm{~mJ}, f=1$ $\mathrm{m})$; the cell was equipped now with two $0.2-\mathrm{mm}$ thick quartz glass windows, to avoid gas turbulence at the open ends with the high He partial pressures. He has a very small $n_{2}$ [33]. Fig. 2.3.8.1a shows the supercontinuum spectra of the output beam for increasing Ar partial pressure $p_{\text {Ar. }}$. At low $p_{\text {Ar }}$ no significant self-focusing and filamentation takes place in the cell and therefore the supercontinuum is of moderate width and only third harmonic radiation (TH) is generated in the UV region of the spectrum, at $270 \mathrm{~nm}$ (box in Fig. 2.3.8.1a). This TH is already observed with the smallest $p_{\text {Ar }}$. Its yield is not noticeably affected by raising $p_{\mathrm{Ar}}$, whereas the supercontinuum broadens, until it covers the TH.

More details in the TH region are shown in Fig. 2.3.8.1b-c. These spectra are measured with a UV spectrometer after reflection from three 270-nm dielectric mirrors (black box in Fig. 2.3.8.1.a). The TH is obviously not shifted or broadened; the second maximum that emerges at $>270 \mathrm{~nm}$, growing at the beginning and then merging with the TH peak, is probably the product of the reflection spectrum of the mirror with the spectral edge of the supercontinuum.

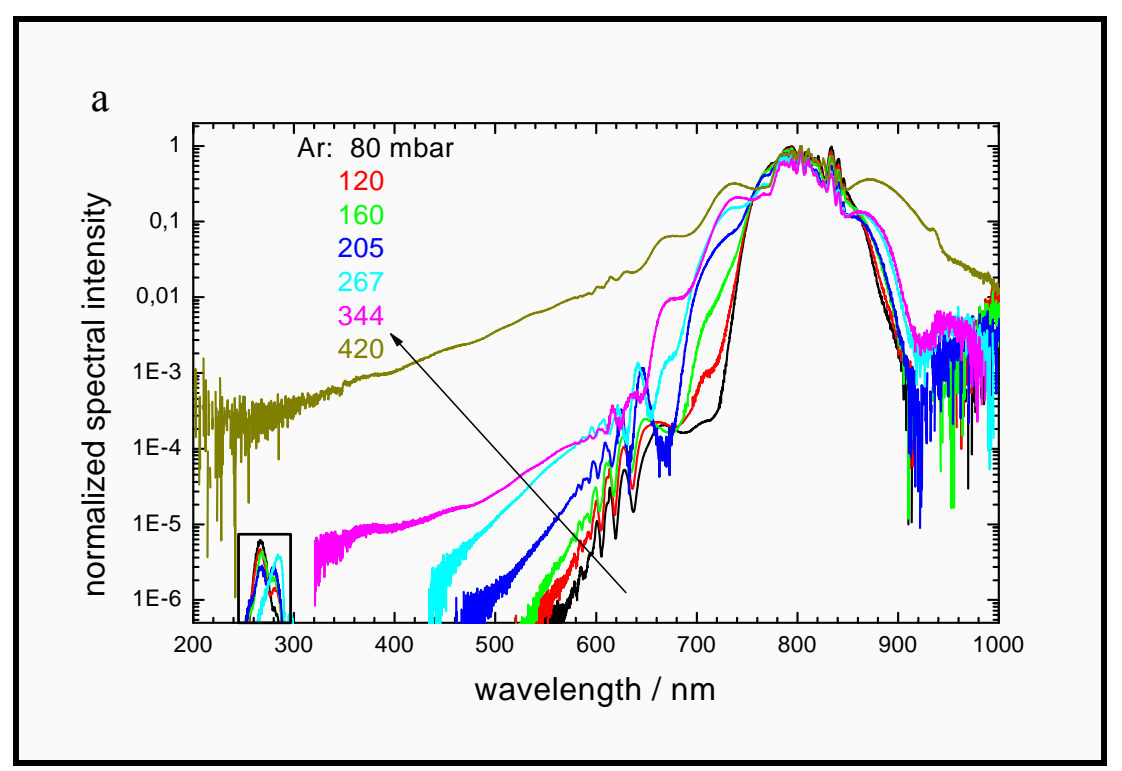




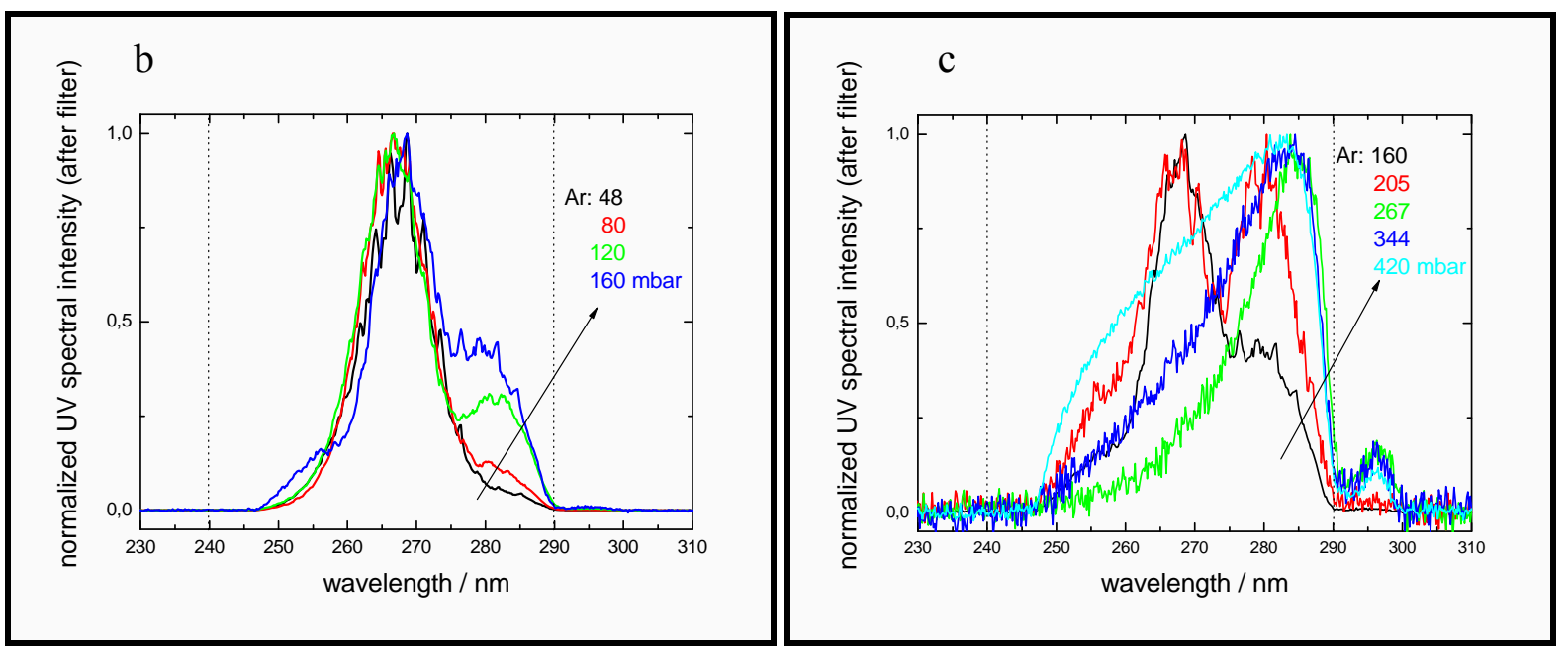

Fig. 2.3.8.1 Supercontinuum spectra and spectra around the third harmonic obtained with increasing Ar partial pressure.

Fig. 2.3.8.2 shows the UV energy within the $240-290 \mathrm{~nm}$ region covered by the dielectric mirrors; the inset shows a magnification of the lower-pressure part. One can observe that for very low $p_{\mathrm{Ar}}$ the TH already appears and slightly grows with increasing Ar pressure. Then it decreases again due, obviously, to phase mismatch, whereas the supercontinuum steadily broadens and rises until it reaches the TH and finally covers it, for $p_{\mathrm{Ar}}>300 \mathrm{mbar}$. The rise of the UV yield at higher $p_{\text {Ar }}$ is therefore only due to the supercontinuum and not the third harmonic.

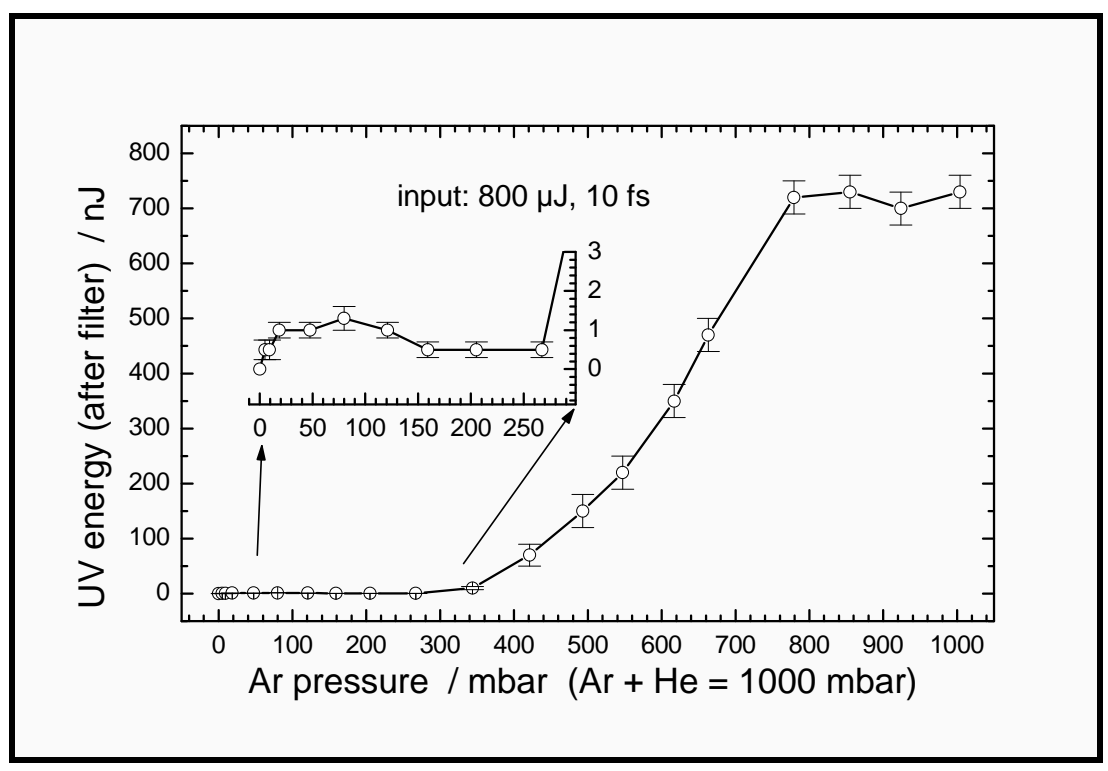

Fig. 2.3.8.2 UV energy within the $240-290 \mathrm{~nm}$ region, with the inset showing a magnification of the lowerpressure part; the nonmonotonic part (maximum at 50-100 mbar) reflects the TH part in Fig. 2.3.8.1b and the sudden rise above 300 mbar is caused by the $\mathrm{SC}$ reaching this spectral region. 


\subsubsection{Different gases}

The effect of the non-linear medium on supercontinuum generation through filamentation was studied by exchanging Ar with air or $\mathrm{N}_{2}$. Fig. 2.3.9.1a shows the spectra obtained after the second Ar cell was now filled with air or $\mathrm{N}_{2}$ at ambient pressure. The pulse duration of the input beam is $10 \mathrm{fs}$ and the energy and focusing conditions the same as in section 2.2 $(E=0.3 \mathrm{~mJ}, f=1 \mathrm{~m})$. Fig. 2.3.9.2b shows the supercontinuum spectra obtained for the different gases and for initial pulse duration $45 \mathrm{fs}$, focal length $f=2 \mathrm{~m}$ and input energy $E=$ $0.89 \mathrm{~mJ}$. In both cases (10-fs and 45 -fs input pulses) argon seems to be the most efficient nonlinear medium for supercontinuum generation, extending the UV cut-off wavelength down to about $250 \mathrm{~nm}$ with both 10 and $45 \mathrm{fs}$. With the 10-fs pulses the spectrum extends approximately to 280 and $320 \mathrm{~nm}$ in $\mathrm{N}_{2}$ and air, while the difference between the gases is much greater for the 45 -fs pulses, which also includes He in the comparison.
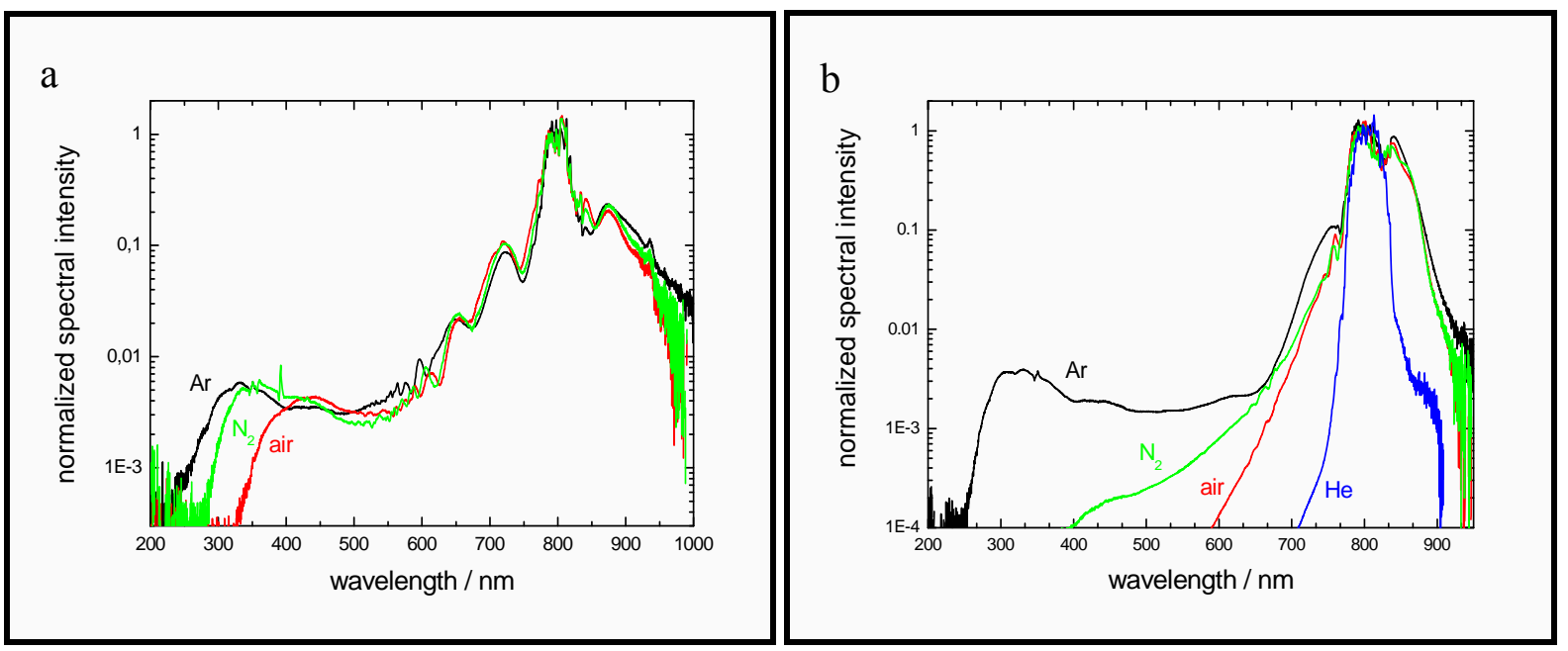

Fig.2.3.9.1 (a) Supercontinuum spectra generated in Ar, $\mathrm{N}_{2}$ and air from the 10-fs, 810-nm pulses with focal length $f=1 \mathrm{~m}$; (b) Supercontinuum spectra generated in Ar, $\mathrm{N}_{2}$ and air from the 45-fs, 800-nm pulses with $f=2$ m.

\subsection{Pulse duration and compression in the $270-320 \mathrm{~nm}$ region of the supercontinuum}

The supercontinuum generated from few-cycle pulses would be a highly attractive source of tunable-wavelength radiation for time-resolved spectroscopy, among other applications, if the pulses at the new frequencies (especially in the UV) were also short or could be compressed. Therefore we investigated pulse compression of spectral parts in the UV, generated by the 17fs, 800-nm pulses into Ar at ambient pressure (Fig. 2.3.4.2b, conditions similar as in section 
2.3.4, input energy $E \leq 0.4 \mathrm{~mJ}$ ). A pair of dielectric mirrors is used to cut out the UV part around $280 \mathrm{~nm}$. The energy of the UV pulses (measured after an additional pair of such mirrors) is $600 \mathrm{~nJ}$ and the bandwidth is $21 \mathrm{~nm}$, centred at $282 \mathrm{~nm}$. Then, the UV radiation is guided via a quartz glass lens $(f=0.5 \mathrm{~m})$ into a prism compressor, consisting of two Brewsterangle $\mathrm{CaF}_{2}$ prisms, a planar aluminium mirror, placed at the focal plane of the lens and an optional slit near the mirror, serving to limit the spectral bandwidth (Fig. 2.4.1); limiting the halfwidth of the UV beam further to $9 \mathrm{~nm}$ (by a slit after the prisms) results in $300 \mathrm{~nJ}$ of energy at $282 \mathrm{~nm}$. The purpose of the compressor is to compensate for both the intrinsic chirp with which the supercontinuum radiation is generated and the dispersion of the long optical path ( $2.5 \mathrm{~m}$ of air and about $6 \mathrm{~mm}$ of quartz glass and $\mathrm{CaF}_{2}$ ). After the prism compressor the UV beam is directed into the ionization chamber of a mass spectrometer (through a $0.5-\mathrm{mm}$ thick $\mathrm{CaF}_{2}$ window), where it is combined with the probe pulses; its duration is determined by cross-correlation with the $17-\mathrm{fs}, 800-\mathrm{nm}$ probe beam, using two-colour transient ionization of Xe (ionization energy $12.15 \mathrm{eV}$ ), which involves absorption of one UV (pump) and five IR (probe) photons.

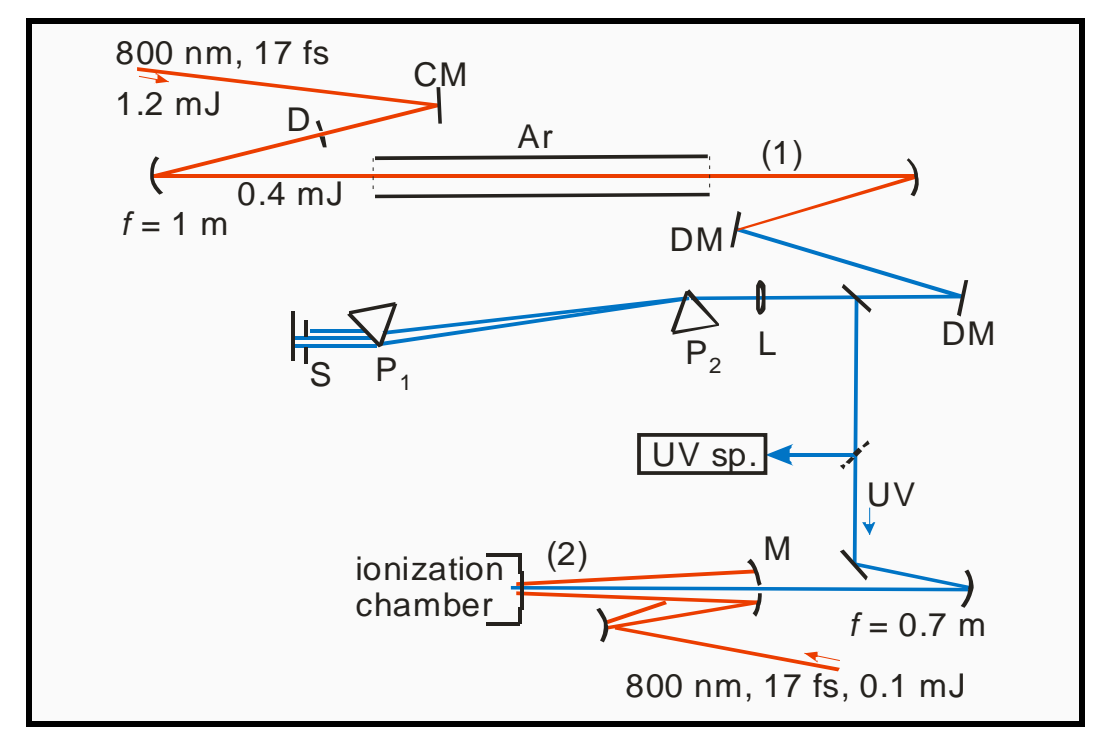

Fig. 2.4.1 Set-up for the generation of ultrashort UV pulses from the supercontinuum spectrum. The gas cell contains slowly flowing argon at ambient pressure (950 mbar); CM: chirped mirror, DM: dielectric mirror, $\mathrm{P}_{1}$, $\mathrm{P}_{2}: \mathrm{CaF}_{2}$ prisms, D: diaphragm of variable diameter, $\mathrm{S}$ : optional slit or razor blade, $\mathrm{L}$ : lens $f=0.5 \mathrm{~m}, \mathrm{M}$ : focusing mirror for the probe radiation, which contains a hole of $4 \mathrm{~mm}$ diameter, so that the UV beam can pass through.

Fig. 2.4.2 shows the cross-correlation, i.e. the $\mathrm{Xe}^{+}$ion yield determined as a function of the delay time between the UV and IR pulses; this set-up is the same as we typically use for pump-probe spectroscopy of ultrafast chemical dynamics [34]. From the $\mathrm{Xe}^{+}$signal (31 fs 
FWHM) the UV temporal width is calculated to be 28 to 30 fs no matter whether the spectral width is 21 or $9 \mathrm{~nm}$ (the two transform limits assuming a Gaussian spectral profile would be around 6 and 13 fs respectively). The same pulse durations were obtained with other wavelengths in the region from 270 to $320 \mathrm{~nm}$, as well as with the third harmonic (TH) generated in a short Ar cell (see sec. 2.5). Since it is known that immediately after such a short path in the nonlinear medium the $\mathrm{TH}$ is at least as short as the fundamental pulse [35], the longer duration measured must be the result of third-order dispersion of $\mathrm{CaF}_{2}$, air and the prism compressor in this wavelength region.

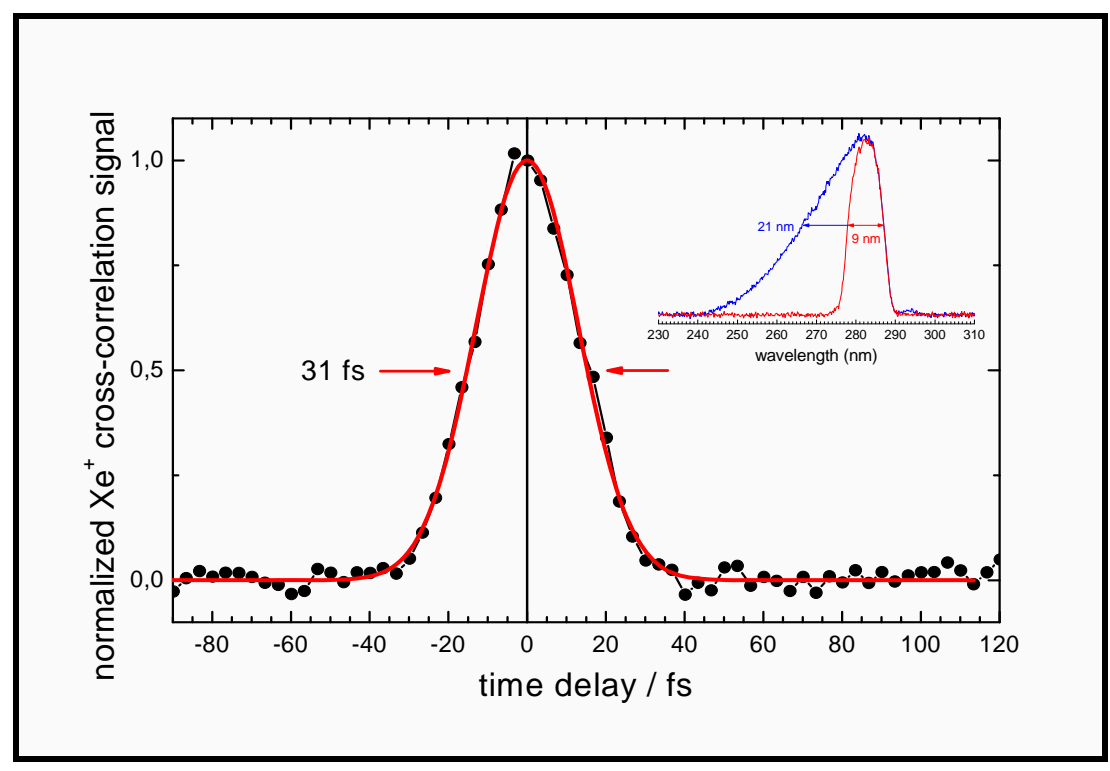

Fig. 2.4.2 $\mathrm{Xe}^{+}$cross-correlation signal ( $\mathrm{FWHM}=31 \mathrm{fs}$ ). The inset shows the UV spectra cut from the supercontinuum in the region of $280 \mathrm{~nm}$ after reflection from four dielectric mirrors (21 nm FWHM) and further narrowed down by a slit after the prisms ( $9 \mathrm{~nm}$ FWHM); the UV pulses from both spectra were compressed to $30 \mathrm{fs}$ FWHM, if we assume that Xe is ionized by $1 \mathrm{UV}+5$ IR photons. The assumption is supported by analogous measurements in Ar.

We therefore prepared the set-up of Fig. 2.4.3, where the radiation continues its propagation only through vacuum $(\leq 4$ mbar) after leaving the filament region (through a $0.6-\mathrm{mm}$ aperture) of the Ar cell where the supercontinuum is generated. The short fundamental $810-\mathrm{nm}$ pulses emerging from the first nonlinear cell filled with 500 mbar Ar (not shown here) have a duration of around $12 \mathrm{fs}$ after the chirped-mirror compressor (reflection from four chirped mirrors like in section 2.1, Fig. 2.1.1). Part of this radiation with energy of $0.7 \mathrm{~mJ}$ was guided to the second Ar cell (Fig. 2.4.3) (the rest is used as the probe radiation in a pump-probe scheme where the generated UV is the pump radiation) and after reflection from additional chirped mirrors (to compensate for the dispersion in air before the second Ar cell) the energy 
is limited by a diaphragm D (Fig. 2.4.3) to approximately $0.35 \mathrm{~mJ}$ to avoid multiple filamentation. In this cell (with variable pressure) the filament is terminated by the pressure drop at the aperture A of $0.6 \mathrm{~mm}$ diameter (the first of four apertures), which is placed $106 \mathrm{~cm}$ from the focusing mirror $(2-3 \mathrm{~cm}$ before the end of the filament); the radiation then passes through three more apertures $(0.7,1$ and $2 \mathrm{~mm})$, serving for differential pumping, and reaches the evacuated box B, where it is recollimated and focused by dielectric mirrors to the massspectrometer and merged with the $810-\mathrm{nm}$ probe pulses for temporal characterization. The pressure drops from a few mbar after the Ar cell to $\sim 5 \times 10^{-6}$ mbar in the ionization region of the spectrometer.

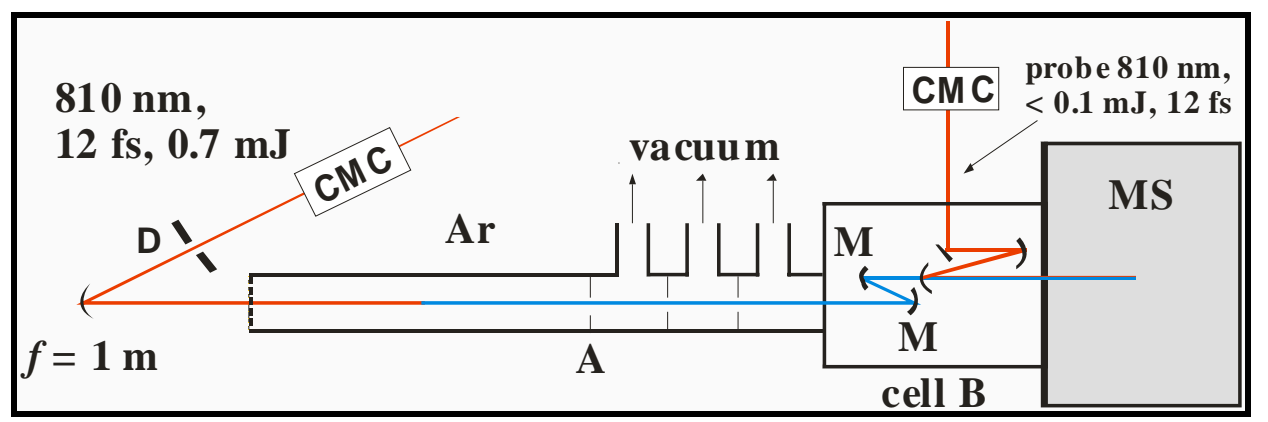

Fig. 2.4.3 Set-up for the generation of the supercontinuum and the short UV pulses. CMC: chirped-mirror compressor, D: diaphragm of variable diameter, A: the first of four apertures serving for differential pumping, B: is an evacuated box with mirrors for collimation, focusing and merging the UV and IR beams, M: dielectric mirrors, MS: mass-spectrometer.

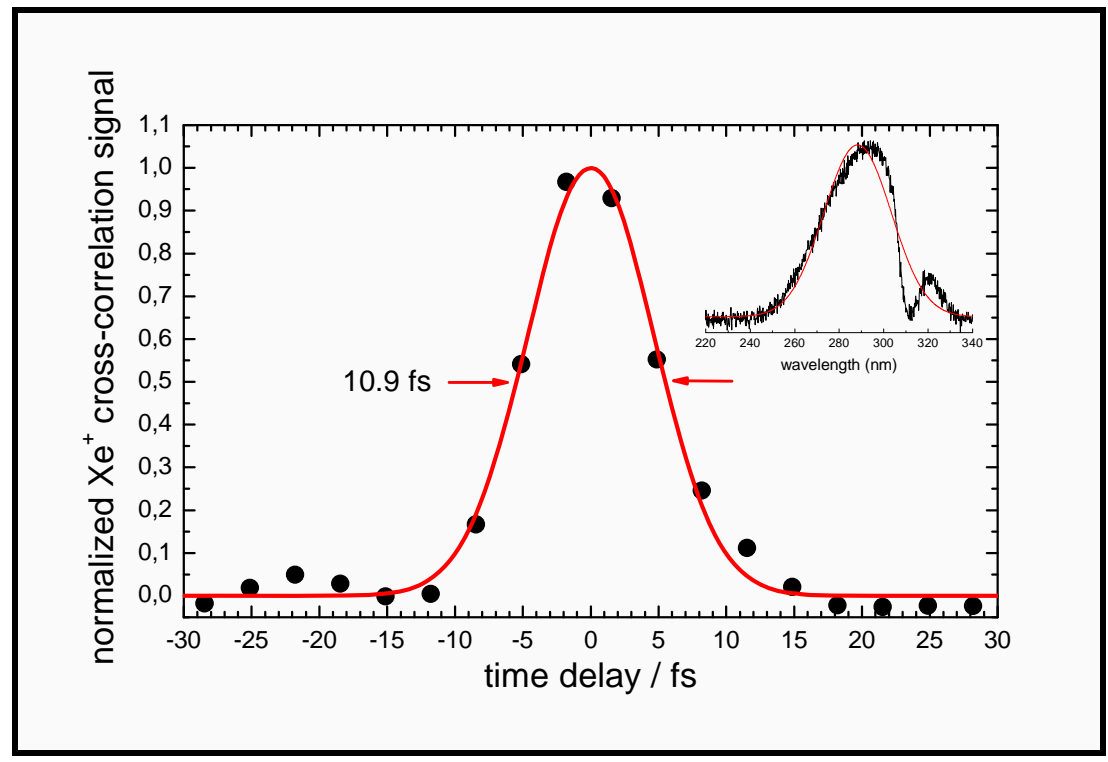

Fig. 2.4.4 Xe $\mathrm{X}^{+}$cross-correlation signal; the inset shows the spectrum of the supercontinuum near $290 \mathrm{~nm}$ after reflection from two dielectric mirrors. 
Fig. 2.4.4 shows the cross-correlation ( $\mathrm{Xe}^{+}$yield) between the UV part of the supercontinuum, cut around $290 \mathrm{~nm}$ (inset of Fig. 2.4.4; the UV spectra are recorded after deflection of the beam out of the box B by a movable mirror), and the fundamental $810-\mathrm{nm}$ pulses. The half-width of $\mathrm{Xe}^{+}$signal is $10.9 \mathrm{fs}$, corresponding to a UV pulse duration of $9.7 \mathrm{fs}$, assuming ionization of Xe by $1 \mathrm{UV}+6$ IR photons; this is $\sim 2.5$ times the transform limit. Some GDD must arise from the dielectric mirrors as well as from the last few centimetres of propagation in the filament region.

\subsection{Third- and fifth-harmonic generation}

Using the set-up of Fig. 2.4.1 the third harmonic (TH) generated in Ar from the 800-nm, 12-fs pulses was also investigated and a pulse duration of about $28 \mathrm{fs}$ was obtained (no lens in the compressor). The second glass cell was now equipped with two 0.2-mm thick, quartz-glass windows and a stainless-steel cell, $18 \mathrm{~mm}$ long, placed in the focal region $(f=1 \mathrm{~m})$; the cell has 2 pinholes of $0.7-\mathrm{mm}$ diameter and contained 200 mbar of flowing Ar, pumped away via the glass cell (background pressure 5 mbar). The pump radiation has an energy of $\leq 1 \mathrm{~mJ}$ and the $\mathrm{TH} \leq 1 \mu \mathrm{J}$ at $269 \mathrm{~nm}(0.1 \%$ conversion efficiency), with $9 \mathrm{~nm}$ spectral half-width. In the case of the UV radiation from the supercontinuum at $282 \mathrm{~nm}$, the duration of the TH (28 fs) achieved after propagation and compression reflects the limit of the prism compressor, since it is known that immediately after its generation in the short cell the TH is as short as the fundamental pulses [35]. The 30-fs TH radiation was used as a pump for the time-resolved spectroscopic study of the dissociation of transition-metal complexes (Chapter 6).

To overcome the limit of this compression scheme, probably caused by uncompensated third-order dispersion of air and optical elements, the experimental set-up of Fig. 2.4.3, with vacuum beam path, is employed for harmonic generation. The short 18-mm Ar cell is now placed in the glass cell of Fig. 2.4 .3 (see Fig. 2.5.1); this setup for TH and fifth-harmonic (FH) generation is the same for the two processes, supplemented by a monochromator for the measurements of the FH spectra. The $800-\mathrm{nm}$ pulses (12 fs, $700 \mu \mathrm{J})$ used to generate the TH pulses are focused $(f=1 \mathrm{~m})$ through the short Ar cell placed in the focus. The calculated intensity in the focus is $1.9 \times 10^{14} \mathrm{~W} \mathrm{~cm}^{-2}$ and twice the Rayleigh length $78 \mathrm{~mm}$. After the cell, the beam propagates under vacuum through several apertures, supporting differential pumping of the chambers (see also section 2.4 and [4]), to the ionization region of the mass spectrometer, where the $\mathrm{Xe}^{+}$cross-correlation scheme is employed between the UV and the IR beam; the UV spectra are recorded after deflection of the beam out of the box B by a movable mirror. 
Fig. 2.5.2 shows the $\mathrm{Xe}^{+}$cross-correlation signal; assuming that ionization of $\mathrm{Xe}$ is caused by the absorption of $1 \mathrm{UV}+5 \mathrm{IR}$ photons we calculate the pulse duration for the TH to be about 10 fs (transform limit $7.7 \mathrm{fs}$ ). These pulses were used as the pump in the pump-probe experiments investigating the dynamics of the ring opening of cyclohexadiene (Chapter 5) and also in some measurements of the dissociation of transition metal carbonyls [8].

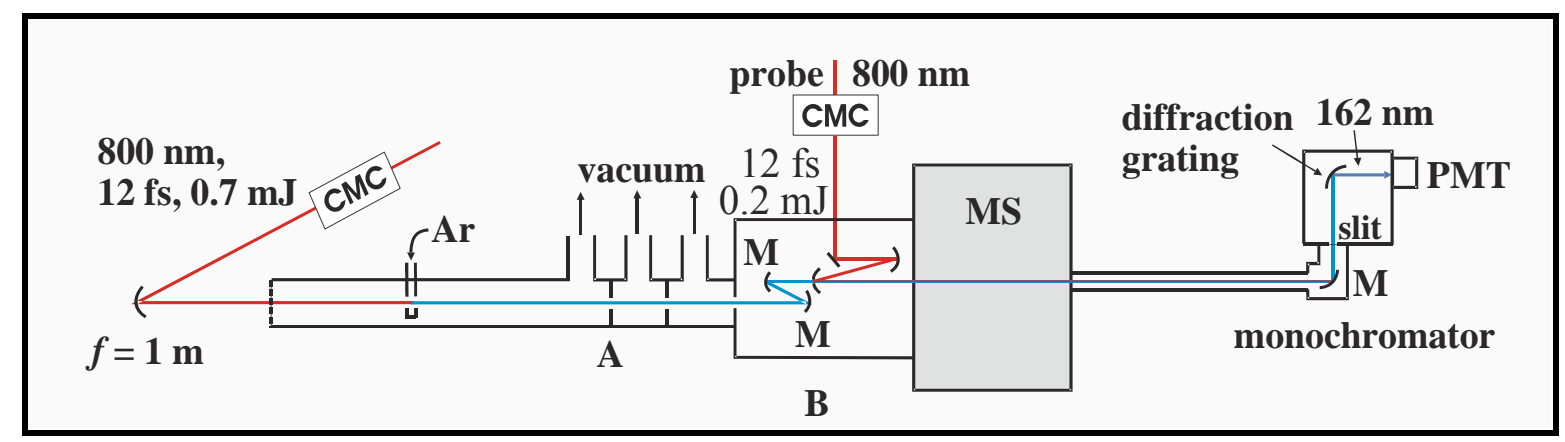

Fig. 2.5.1 Set-up for the generation of the TH and FH. The entrance window $\left(\mathrm{CaF}_{2}\right)$ is $0.5 \mathrm{~mm}$ thick. The probe pulses used for cross-correlation in the time-of-flight mass spectrometer (MS) merge with the harmonic beam at a mirror with a hole, CMC: chirped-mirror compressor, B is an evacuated box with mirrors for collimation, focusing and merging the beams, M: dielectric mirror at $270 \mathrm{~nm}(160 \mathrm{~nm})$ for guiding the $\mathrm{TH}(\mathrm{FH}) \mathrm{ra}-$ diation into the MS for the time-resolved measurements, replaced with aluminium mirror for the spectral measurements.

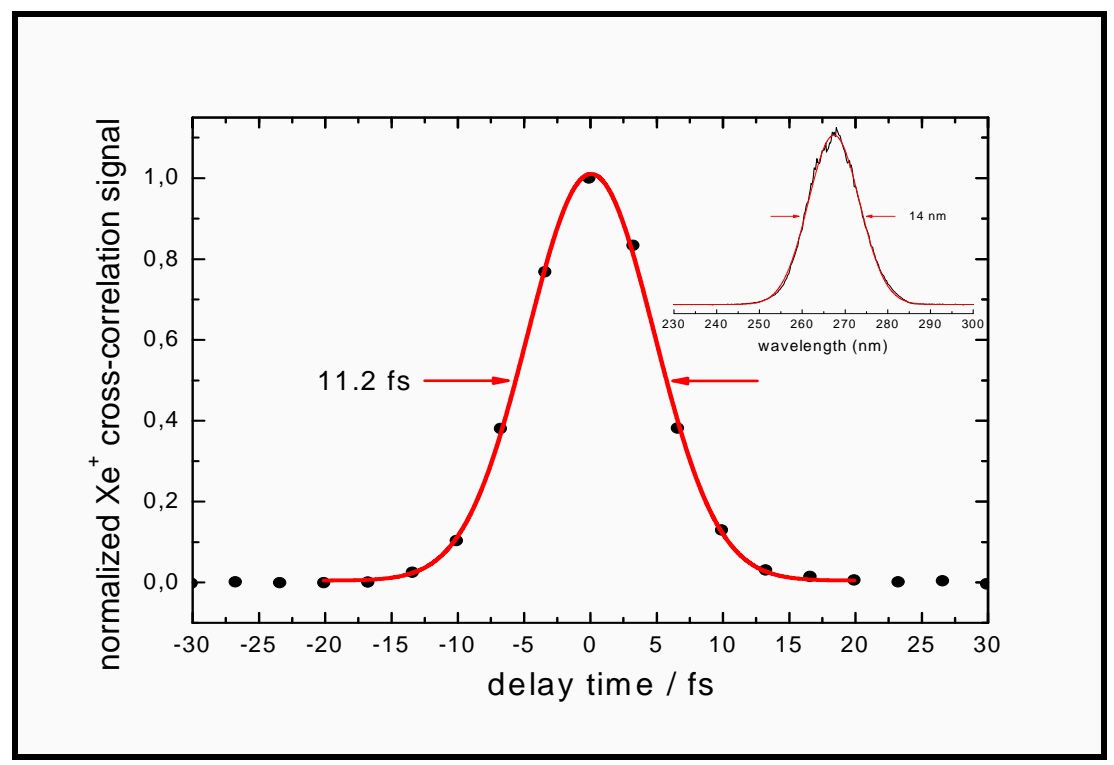

Fig. 2.5.2 $\mathrm{Xe}^{+}$cross-correlation signal. The inset shows the corresponding $\mathrm{TH}$ spectrum.

The $800-\mathrm{nm}$ pulses $(12 \mathrm{fs}, 710 \mu \mathrm{J})$ used to generate the FH pulses $(162 \mathrm{~nm})$ are focused $(f$ $=1 \mathrm{~m}$ ) through the same short Ar cell. After the cell, the beam propagates under vacuum through several apertures supporting differential pumping of the chambers (details above and 
in [4]), through the ionization region of the mass spectrometer and through a $\mathrm{CaF}_{2}$ window (2 $\mathrm{mm}$ ) to a scanning monochromator (Acton Research, Model VM-502), which records the spectra. No transmissive optics is used between the Ar cell and the mass spectrometer; three aluminum mirrors are used to guide the fifth harmonic to the spectrometer. The pressure drops from a few mbar after the Ar cell to $\sim 5 \times 10^{-6}$ mbar in the ionization region and is $<5 \times 10^{-4}$ mbar in the monochromator. Spectra of both the fifth and the third harmonic are recorded.

Fig. 2.5.4 shows the spectra of the third and fifth harmonic. Their shapes do not change with the Ar pressure $p_{\mathrm{Ar}}$ in the short cell. The energies as a function of $p_{\mathrm{Ar}}$ are shown in Fig. 2.5.5. For the third harmonic, they are measured directly, replacing the monochromator by a powermeter (in air); these data then served for calibrating the monochromator readings at the fifth harmonic. The energies initially grow with $p_{\mathrm{Ar}}^{2}$, as expected in the perturbative limit, reaching $140 \mathrm{~nJ}$ at $270 \mathrm{~nm}\left(2 \times 10^{-4}\right.$ of the input) around $160 \mathrm{mbar}$ and $4 \mathrm{~nJ}$ at $162 \mathrm{~nm}\left(6 \times 10^{-6}\right.$ of the input) around 50 mbar. Within a factor of 2 , the ratio of the two pressures (160 mbar/50 mbar $=3.2$ ) reflects the refractive-index difference at the two wavelengths compared to the fundamental, $5\left(n_{162}-n_{810}\right) / 3\left(n_{270}-n_{810}\right)=6$. (Any plasma contribution to the refractive index is not taken into account.). This means that the efficiency decrease at higher $p_{\text {Ar }}$ is caused by the loss of phase matching. It is remarkable that at 50 mbar the yield of the VUV radiation is by only a factor of 10 smaller than that of the third harmonic, while this factor even decreases to 2 at $<20$ mbar. The ratio of the spectral half widths of the fundamental and the fifth harmonic $\Delta v_{162} / \Delta v_{810}=57 \mathrm{THz} / 37 \mathrm{THz}=1.54$, which is by $30 \%$ smaller than $\sqrt{ } 5$, the value expected in the perturbative limit. (The bandwidth $-63 \mathrm{THz}-$ of the third harmonic in Fig. 2.5.4 is by almost a factor of $\sqrt{ } 3$ larger than that of the fundamental.)

The maximum energy of the fifth harmonic $(\approx 4 \mathrm{~nJ}$ at $162 \mathrm{~nm})$ is derived from the comparison of the two spectra of Fig. 2.5.4, taking into account the efficiency of the diffraction grating and the photomultiplier and the estimated reflectivity of the three $\mathrm{Al}$ mirrors $(\approx 50 \%$ each at $162 \mathrm{~nm}$ ) in Fig. 2.5.1. This value is only a lower bound of the feasible energy: the 270-nm energy measured directly after the Ar cell is up to $1 \mu \mathrm{J}$ (as also in [4]), 7 times higher than what arrives at the monochromator. Obviously the losses are caused by the apertures, which served for maintaining the differential pumping. Although their diameters were large enough to transmit both the fundamental and the harmonics beams, the possibility for their alignment was limited in the stiff vacuum setup. Optimizing the design for maximum optical transmission could thus improve the energy by up to a factor of 7 . 


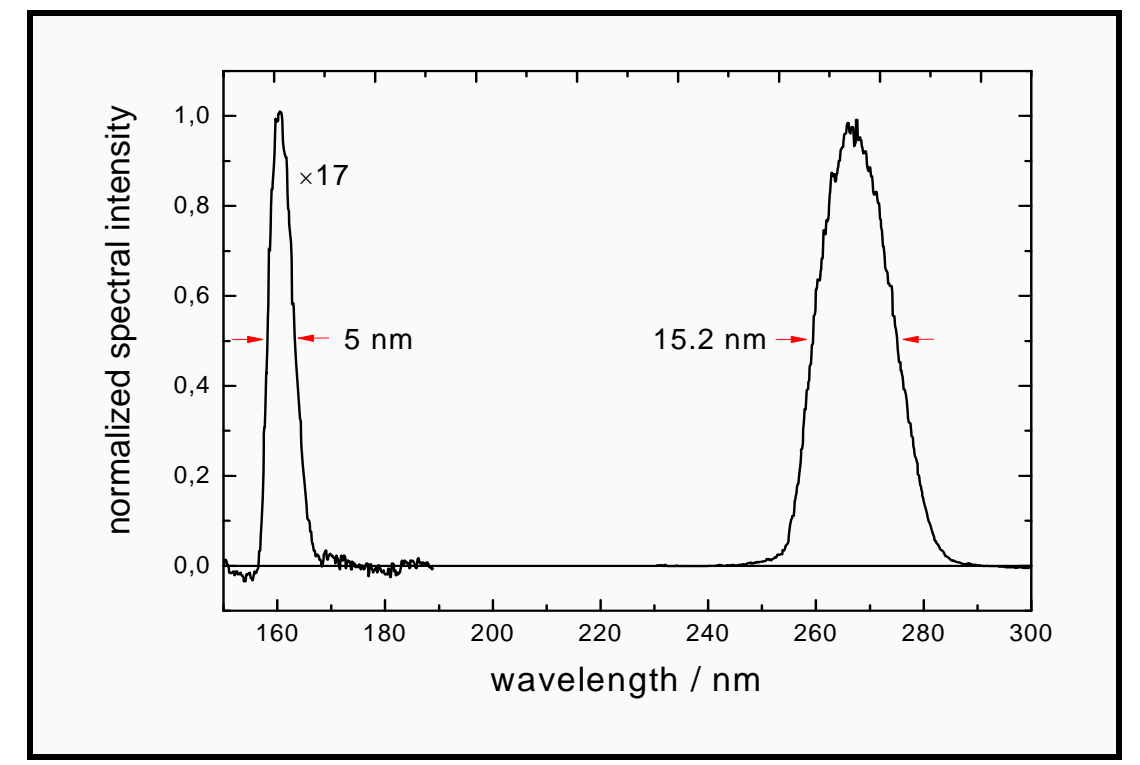

Fig. 2.5.4 Fifth- and third-harmonic spectra recorded at $20 \mathrm{mbar}$ of Ar in the short cell.

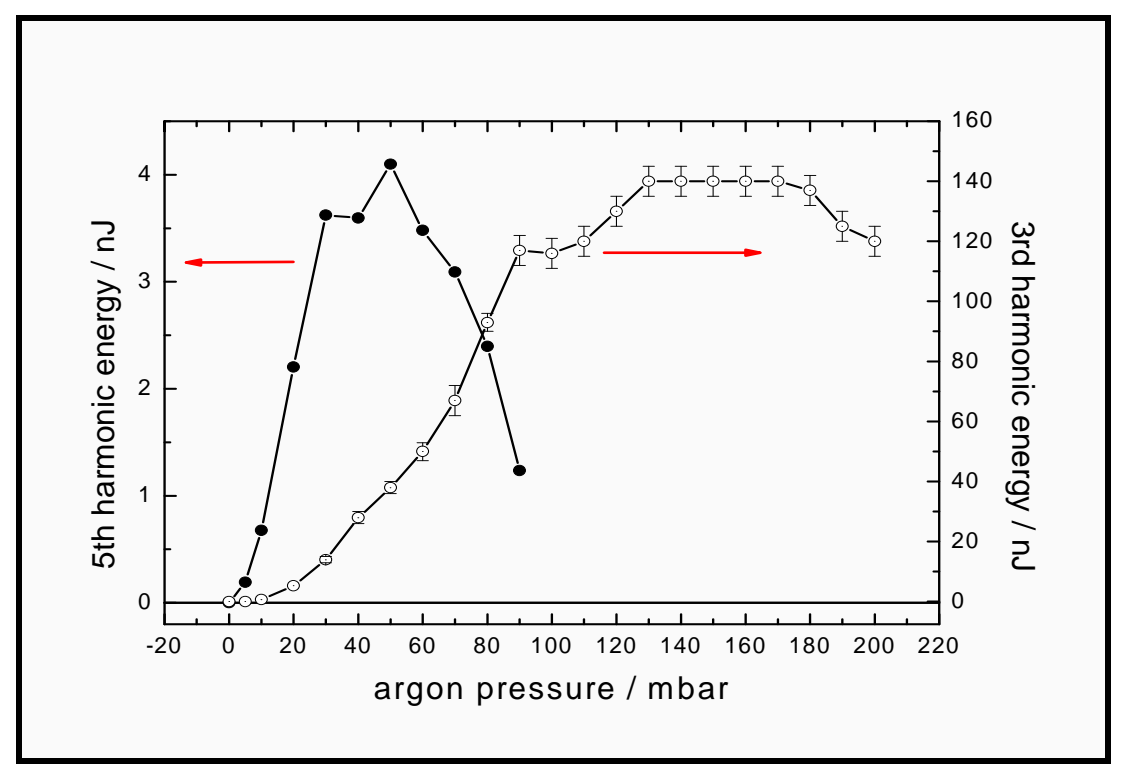

Fig. 2.5.5 Pulse energies at the fifth (full circles) and third (open circles) harmonic.

The spectral half-width of the fifth harmonic pulses $(\sim 5 \mathrm{~nm}(57 \mathrm{THz}))$, would sustain a duration of $\sim 7.7 \mathrm{fs}$. We apply the cross-correlation scheme, where Xe is ionized by absorption of one VUV $(7.65 \mathrm{eV})$ and three IR $(1.53 \mathrm{eV})$ photons, to determine the duration of the VUV pulses. The two curved dielectric mirrors that replace now M in chamber B (Fig. 2.5.1) suppress the third harmonic $(3 \omega)$ and fundamental $(\omega)$ by a factor of $>100$, so that ionization by $3 \omega+5 \times \omega$ or $2 \times 3 \omega+2 \times \omega$ is negligible. Also the pump-probe experiments with molecules, resonant at $5 \omega$, confirm that the measured pulse duration by the $\mathrm{Xe}^{+}$cross-correlation is that with $5 \omega$. Furthermore the time-resolved $\left(\mathrm{Xe}^{+}\right)$signals show the $p_{\mathrm{Ar}}$ dependence as that of the 
fifth harmonic in Fig. 2.5.5. Fig. 2.5.6 shows this Xe ion signal as a function of the delay time between the VUV and the fundamental pulses as well as the cross-correlation fitting curve resulting from the first power of the VUV and the third power of the 810-nm pulses. The temporal half-width of the $\mathrm{Xe}^{+}$signal is $\approx 13 \mathrm{fs}$, so that the fifth-harmonic pulse duration is $11 \pm 1$ fs. This corresponds to $\sim 1.4$ times the transform limit, whereas the 12-fs fundamental pulses are transform limited. A chirp of the VUV pulses might be caused by cross-phase modulation with the fundamental, and an additional lengthening by the residual group delay dispersion of the dielectric mirrors; these mirrors have not been optimized for minimum dispersion (as was also the case for the third harmonic in [4]). A further source of a chirp can be intrinsic in harmonic generation [36]. Sekikawa et al., using a setup with the focus after a Xe cell, obtained a negative chirp at $160 \mathrm{~nm}$ and used it for pulse compression by a normally dispersive material (LiF) [36].

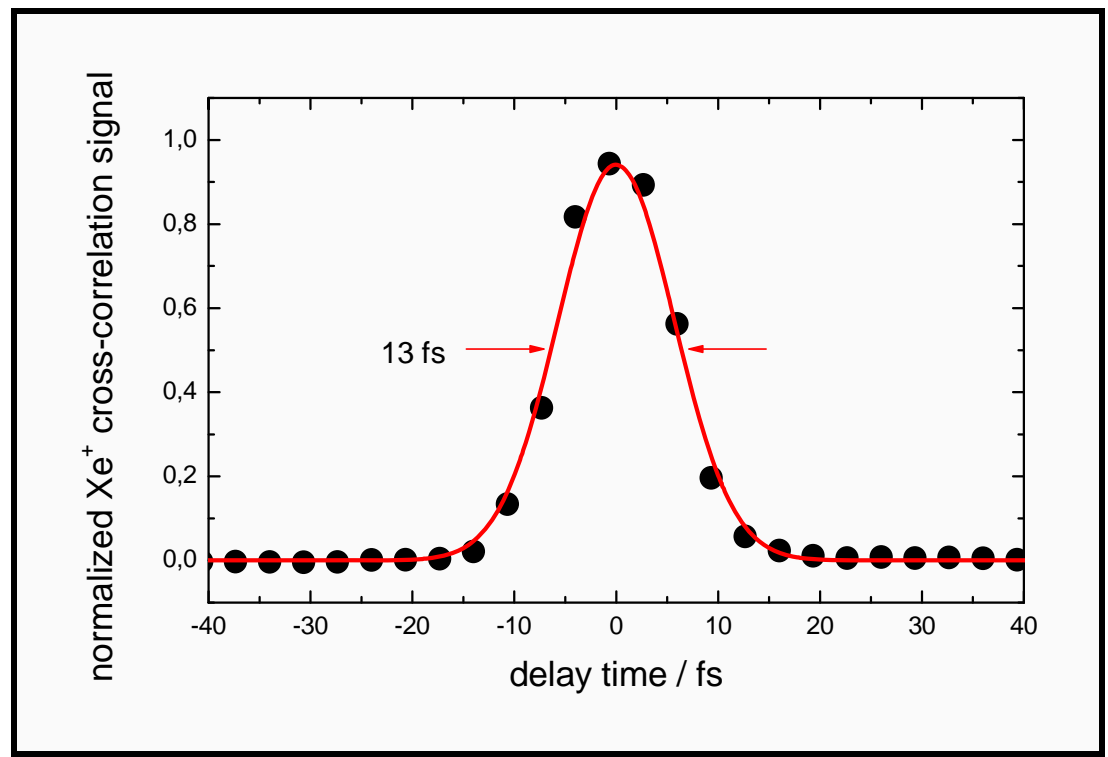

Fig. 2.5.6 Cross-correlation of the fifth harmonic and IR pulses, resulting from ionization of Xe by 1 VUV + 3 IR photons. The smooth curve is a fit using Gaussians for the VUV and IR pulses.

\subsection{Discussion}

The demonstration of a very broad supercontinuum spectrum from $\geq 1000$ to $250 \mathrm{~nm}$, with a simple setup, starting from $1 \mathrm{~mJ}$ of Ti-sapphire laser radiation (Fig. 2.2.2), is very remarkable and is attractive for applications. Previously the supercontinuum, generated from ultrashort pulses (usually between 50 and $150 \mathrm{fs}$ ) with wavelength around $800 \mathrm{~nm}$, typically attained $350 \mathrm{~nm}$ at best, and this limit has been attributed to intensity clamping in self-focusing [37]. Only at substantially higher energies, spectra of similar width have been reported: starting 
from $>200 \mathrm{~mJ}$, a continuum reaching down to $150 \mathrm{~nm}$ was obtained [31, 33] and a width similar to ours was found by focusing up to $10 \mathrm{~mJ}$ in air [38]; multiple filamentation was observed in these works. It can be assumed that, having lost part of the spatial coherence, the pulses could not remain short either. Here it was shown that under conditions of single filaments similar spectral widths can be obtained with much less energy, if the initial pulses are compressed to $\leq 10$ fs before; the parameters influencing the spectral features of the supercontinuum radiation are also identified. Single filaments are indispensable for good pointing stability of the output beam and probably for obtaining short pulses too.

The physical mechanisms that are mainly expected to contribute to the spectral broadening of the laser beam towards the UV are self-phase modulation (SPM) by the optical Kerr effect in the (self-steepened $[17,38]$ ) trailing edge of the fundamental pulse and the self-induced plasma, which produces frequencies that are only blue-shifted (see also Chapter 1, sec. 1.2.4). With a Gaussian pulse, Kerr-effect SPM extends the spectrum symmetrically around the central frequency of the input spectrum; self-steepening, however, causes an abrupt slope in the falling part of the pulse so that SPM actually leads to an asymmetric broadening, in favour of short wavelengths. Without this effect the one-sided extension from $810 \mathrm{~nm}$ towards the UV (Chapter 1, sec. 1.2.2) would be [39] $\Delta v_{1}=\Delta \omega / 2 \pi=1.43\left(n_{2} I_{0} / \lambda_{0}\right)\left(z / t_{\mathrm{p}}\right)$. Inserting the peak intensity on the axis $I_{0}=47 \mathrm{TW} / \mathrm{cm}^{2}$, the pulse length $t_{\mathrm{p}}=10 \mathrm{fs}$ and for the filament length $\mathrm{z} \approx$ $15 \mathrm{~cm}$, one obtains $\Delta v_{1} \approx 170 \mathrm{THz}\left(\approx 5700 \mathrm{~cm}^{-1}\right)$; this is much smaller than the measured frequency spread $(810-250 \mathrm{~nm})$ of $27600 \mathrm{~cm}^{-1}$. This discrepancy indicates that self-steepening and perhaps plasma contributions to $\Delta v_{1}$ have a drastic effect. Because the intensity is large, higher-order nonlinearities have been claimed to contribute to the refractive index (namely a term $n_{3} I^{2}$ besides the lowest nonlinear term $n_{2} I$ ) and thus to SPM [40]. A criterion for the importance of higher-order terms is the ratio (0.03) of the optical field strength $(1.9 \mathrm{~V} / \AA$ at 47 $\left.\mathrm{TW} \mathrm{cm}^{-2}\right)$ over the atomic unit of field strength $(60 \mathrm{~V} / \AA)$. This does not seem very high and so the higher-order terms may be expected to contribute only a limited correction. On the other hand, this intensity definitely causes a non-negligible ionization of argon, a process requiring 10 photons at $800 \mathrm{~nm}$; high-order nonlinear effects can thus not be ignored. The plasma contribution to the spectral broadening is expected to increase a little with shorter pulses, since pulses of the same peak power but shorter duration are expected to have slightly higher onaxis intensity $[2,28]$; comparison of the spectra with 45 and 10 fs shows however that the difference in their widths is much more significant. In contrast, the Kerr-effect phase shift is pro- 
portional to the temporal rate of the intensity change $\left(\propto n_{2} \mathrm{~d} I / \mathrm{d} t\right)$ and thus $\propto t_{\mathrm{p}}^{-1}$. Therefore the broadening in the UV is mainly due to the Kerr effect and the contribution of the plasma is negligible under the present conditions. This confirms Gaeta's prediction [41]. This calculation is based on the non-linear Schrödinger equation including also self-steepening and terms beyond the slowly-varying-envelope approximation (SVEA). Using a similar equation for the calculated spectra in [28] and employing the input conditions of our experiment [1] for the numerical simulations, a semi-quantitative agreement with the UV part of the supercontinuum has been obtained. Among all the main linear and nonlinear effects, plasma generation and self-steepening were also included in the calculations; the latter becomes more important with shorter pulse durations, according to the calculations, and therefore this is an additional reason why the 6-fs pulses extend the UV cut-off farthest to the UV $(210 \mathrm{~nm})$ (besides the scaling of $\Delta v_{1}$ with $\left.t_{\mathrm{p}}^{-1}\right)$.

Although the simple theory for $\Delta v_{1}$ is insufficient to explain the large broadening observed in the UV, it might qualitatively predict scaling on changing parameters; it suggests proportionality to $n_{2} I, t_{\mathrm{p}}^{-1}$ and $z$. The intensity $I$ cannot be raised further, since it is clamped and raising the energy of the incident pulses only leads to break-up into many filaments. But the pulse length seems to be important. The 6-fs pulses in fact give rise to the broadest spectrum; it extends to $210 \mathrm{~nm}$, compared to $250 \mathrm{~nm}$ with $10 \mathrm{fs}$ or $17 \mathrm{fs}$ and $600 \mathrm{~nm}$ with $45 \mathrm{fs}$. But the spectral spread did not increase $\propto t_{\mathrm{p}}^{-1}$. An explanation is provided by the observation (and the calculation in [28]) that with the shorter pulses the filament was also shorter $(\approx 5 \mathrm{~cm}$ with the 6-fs pulses instead of $\approx 15 \mathrm{~cm}$ with 10 -fs pulses; although the estimation by inspection of the luminescing column is probably not very accurate). The key parameter may be the product $z t_{\mathrm{p}}^{-1}$, which varies much less than $t_{\mathrm{p}}^{-1}$ on shortening the pulse. The importance of $z$ is clearly shown by Fig. 2.3.3.2 and 2.3.5.1; the former directly demonstrates that the UV spectrum is more pushed to shorter wavelengths, the longer the radiation propagates at high intensity, while in the latter it can be seen how the looser initial focusing and hence the longer Rayleigh length extends the cut-off of the supercontinuum, generated by the 45 -fs pulses, from $600 \mathrm{~nm}$ with $f=$ $1 \mathrm{~m}$ to $250 \mathrm{~nm}$ with $f=2 \mathrm{~m}$. Also the spectra in [31] extended to $150 \mathrm{~nm}$, with $f=5 \mathrm{~m}$; perhaps $f$ was indeed more important for the supercontinuum spectrum than the high power (that caused multiple filamentation). The effect of the focal length is also known from experiments 
where high powers, giving rise to multiple filaments, are used in atmospheric monitoring (e.g. [42]). On the other hand, $f$ has practically no effect on the spectra of the 10-fs pulses (Fig. 2.3.5.1a).

The filament region indicates the propagation path at high intensities, where practically the nonlinear conversion of the input spectrum takes place. It is thus very important to understand why the filament eventually terminates and why it is shorter in the experiment with the 6-fs pulses. Losses due to absorption in the nonlinear medium or conical emission, which can terminate much longer filaments [43] are not measurable in our case [1]: losses would have to suppress the power to $<P_{\mathrm{cr}}$, i.e. by a factor of 4 (our power was 4 times higher than the critical power for argon), which would be easily measurable. It was predicted, however, in [28] that under the present conditions the pulses tend to split after some propagation, so that the power is reduced to near or below the critical power, which can explain the termination [28]. In some cases pulse splitting and filament termination is attributed to normal group-velocity dispersion (e.g. in [44]). Although under our conditions the dispersion length with the 10-fs pulses is much longer $(1.8 \mathrm{~m})$ than the length of the filament $(15 \mathrm{~cm}$ with the $10-\mathrm{fs}$ pulses $)$, the termination of the filament and pulse splitting turned out to be sensitive to inclusion of the dispersion term in the calculation [28]. Other suggested mechanisms that could be responsible for the termination of the filaments are discussed in [28].

The observation of conical emission (that is of rings surrounding the central beam spot, which contain a larger fraction of the short-wavelength radiation than the centre) in the case of the longer (e.g. $45 \mathrm{fs}$ ) pulses has been attributed to four-wave mixing and other phasematched processes $[14,23]$ or by others to an anti-guiding property of the interface between the plasma and the nonlinear gas [45] or to diffraction by the plasma [46]; these mechanisms assume that the refractive index of the plasma is lower than that of surrounding gas and since this difference is more significant for shorter wavelengths, these are refracted out of the axis. Conical emission was assumed to be characteristic of filamentation and supercontinuum generation $[47,48]$. On the other hand, however, it is not observed with the 10-fs input pulses (the shortest wavelengths are concentrated on the axis), which is also predicted by the calculations in [28]. This could perhaps be explained by the same anti-guiding and diffraction mechanisms mentioned previously. As plasma accumulates during the pulse duration, the above effects might be more pronounced with long pulses, in particular if self-steepening is also taken into account: A balance between Kerr-effect focusing and plasma-induced defocus- 
ing may be reached near the maximum of the pulse; later on the plasma density might increase (if self-steepening is not too strong) and the anti-guiding can set in, affecting mainly the short wavelengths. However, with pulses of $\leq 10 \mathrm{fs}$, self-steepening can be quite strong and give rise to pulses with a shock-like temporal decay, so that no more plasma accumulates and the refractive index on the axis will not be sufficiently lowered to values causing conical emission.

Another parameter influencing the spectral features of the supercontinuum was found to be the input pulse energy. Raising the input energy $E_{\mathrm{p}}$ (or power $P$ ) (below the threshold for multiple filamentation, i.e., $\leq 0.3 \mathrm{~mJ}$ ) steadily shifted the UV cut-off to shorter wavelengths (Fig. 2.3.1.1). On first sight this seems intuitive. However, one should remember that the clamped intensity is the same in all cases and the higher powers only expand the beam cross section [2]. It is therefore probably $z$ (the filament and propagation length at high intensity) that increases with power, resulting in more efficient UV generation. This is supported by the observation that $E_{\mathrm{p}}$ has more effect in the UV than in the IR, as expected from the prolongation of propagation, which develops the steepening of the pulse decay (which generates the UV) and a corresponding flattening of the rising edge (which generates the IR). Above the limit for multiple filamentation the UV yield decreases again with further increase of the energy, while the length of the filament is shorter $(5-7 \mathrm{~cm})$ in this case than with a single filament only $(12-$ $15 \mathrm{~cm})$.

The initial chirp and/or pulse duration influence not only the supercontinuum cut-off wavelength, but also the spectral half-width. It turns out that with the 45-fs pulses (transform limit) the broadest half-width is achieved with initially positively-chirped pulses, while a negative chirp causes spectral narrowing (Fig. 2.3.6.1). Usually self-phase modulation (SPM) aims at spectral broadening and pulse compression, but the narrowing found above can be exploited in cases where lengthening is desired. This was indeed recommended on observation of spectral narrowing with negatively chirped pulses by SPM in quartz glass fibers [49]. A gas-filament would exhibit fewer losses than a fiber and permit higher peak powers too. The fact that the strongest broadening of the half-width is observed with a positive initial chirp might be connected to the self-compression and negative chirp found after self-focusing in Ar, with energies higher by a factor of $2.5-5$ [50-52]; the negative chirp induced by the filament perhaps compensates the positive chirp of a long input pulse, shortens it and so SPM is stronger than with transform-limited incident pulses. (On further propagation it would also compensate the 
normal dispersion and cause the self-compression in [50-52]). The negative chirp generated with the higher powers (which require use of lower gas pressures) may be caused by the plasma: the electron density, growing during the pulse, raises the negative contribution to the refractive index; this time-dependence contributes a negative chirp. Note that with higher powers the diameter of the plasma column is larger [2] (the clamped intensity is the same), so that in this case its influence to SPM and the spectrum becomes non-negligible.

In contrast to the maximum half-width, the maximum spread of the output spectrum towards the short wavelength region is achieved when the input pulses are transform-limited (zero initial chirp) (Fig. 2.3.6.1). This is in agreement with the consideration that the Kerreffect broadening of the pedestal increases with increasing $\tau_{\mathrm{p}}^{-1}$, although not proportionally. (Note that the lower initial intensity of the chirped - and hence longer - input pulses will stabilize in the filament to the clamped-intensity value.) Probably self-steepening is perturbed and eventually suppressed already by a moderate (positive or negative) chirp and thus larger temporal widths and therefore the UV cut-off wavelength (the UV is generated in the steepened trailing edge of the pulse) is also influenced. We hence suggest that the UV near the cutoff is generated by the Kerr-effect SPM in the self-steepened trailing edge, whereas the halfwidth is additionally influenced by the plasma. With the 10-fs pulses the effect of the initial chirp is less dramatic (Fig. 2.3.6.2). However, for each curve the input iris of the Ar cell is optimized so as to get a maximum output in the UV. Apparently the effect of the chirp on the cut-off can be partially compensated by varying the input energy.

Replacing Ar by $\mathrm{N}_{2}$ or air shifts the UV cut-off to slightly or moderately longer wavelengths, respectively, with the 10-fs pulses; with the 45-fs pulses the effect is more pronounced (Fig. 2.3.9.1 - 2). The nonlinear index $n_{2}$ and the ionization probability of $\mathrm{N}_{2}$ and Ar are similar and so $\Delta v_{1}$ should not be very different for the two cases. In air, however, the plasma is produced from ionization of oxygen whose ionization energy is smaller than that of Ar or $\mathrm{N}_{2}$. The higher electron density would reduce the clamped intensity in the filament [2], resulting in a certain decrease of the broadening. The effect of the different gases (amount of plasma) is smaller with the 10 -fs pulses than with the 45 -fs pulses; however, the filament length might be different for the respective gases and depend on the pulse duration. The fact that the effect is absent in the half-width might be due to the fact that the input energy and initial chirp have been optimized for the best UV yield for every measurement. These parameters were shown above to influence the spectral cut-off and half-width in a different way. 
An interesting observation in the experiments with the 10-fs pulses is the fact that the third harmonic $(\mathrm{TH})$ does not contribute to the spectral broadening of the supercontinuum towards the UV. Fig. 2.3.8.1 shows that the weak TH appears separately from the supercontinuum at low Ar pressures, while it eventually starts dropping with increasing Ar density. Such a nonmonotonic pressure dependence of the $\mathrm{TH}$ yield is expected, if phase-matching plays a role. The TH is then covered by the expanding supercontinuum (for the generation of which no phase matching seems to be required). We can therefore conclude that the two spectral components have a different origin and it is not the TH that extends the UV cut-off of the supercontinuum to shorter wavelengths. It was previously suggested that TH broadens due to crossphase modulation with the fundamental and the two spectral features then merge $[38,53]$. It is also worth noting that the TH maximum shows up at the expected wavelength $(270 \mathrm{~nm})$ in contrast to the prediction of a long-wavelength shift [54]. The best TH yield obtained is about $2 \times 10^{-6}\left(\approx 1.3 \mathrm{~nJ}\right.$ with $800 \mu \mathrm{J}$ input, Fig. 2.3.8.2). Much better conversion efficiency $\left(1-2 \times 10^{-3}\right.$ can be achieved by focusing through a short cell $(18 \mathrm{~mm}$ long) filled with about $200 \mathrm{mbar}$ of Ar, with pinholes and He or vacuum outside [3]. The evolution of the short-wavelength part of the supercontinuum spectrum on raising the Ar pressure was also investigated in the scheme where the filament is terminated at a distance of about $106 \mathrm{~cm}$ from the focusing mirror $(f=1$ m, 10-fs input pulses) [4] by a pressure gradient; it was estimated that the contribution of the TH to the $250-300 \mathrm{~nm}$ spectral part is less than 5\% at 900 mbar of Ar.

Another very important feature of the UV light generated from the supercontinuum source is its duration, in particular for its use as the pump radiation in time-resolved spectroscopic studies. Our initial attempt to achieve short pulses was compression by a pair of prisms (section 2.4); the prism compressor should compensate for both the intrinsic chirp with which the UV might be generated inside the filament region and for that introduced by normal dispersion along propagation through some additional Ar, $2.5 \mathrm{~m}$ of air and optical elements (after the end of the filament). However, the shortest duration achieved was $30 \mathrm{fs}$ for both the supercontinuum (at 270 to $320 \mathrm{~nm}$ ) and the third harmonic (generated in a shorter cell, which should not give rise to dispersion). So we conclude that this is a limitation of the prism compressor, which doesn't compensate for the higher-order dispersion in air and optical elements. Since there is evidence that the UV is mainly generated in the self-steepened trailing edge of the pulse by SPM, it might be expected to be no longer than this optical shock. The calculations [28] predicted that the input $800-\mathrm{nm}$ pulses initially get shorter (after $5 \mathrm{~cm}$ of propagation) and then lengthen again and split on further propagation. Therefore, stopping the filament by a 
pressure gradient would prevent the splitting and probably result in short UV pulses. In fact, trying this technique by employing a pressure drop with an aperture, beyond which argon is pumped away, and having the beam propagate under vacuum thereafter, leads indeed to short UV pulses around $290 \mathrm{~nm}$ [4] with duration of about $10 \mathrm{fs}$. Although this is longer than the predicted values from the calculations [28] (which did not study spatial effects such as wavefront distortions), the observation shows that the supercontinuum can be produced within the original pulse duration, obviously because lengthening by pulse splitting is avoided by terminating the filament by a pressure gradient.

The same vacuum set-up was used for third- and fifth-harmonic generation from 12-fs 810$\mathrm{nm}$ pulses, replacing the long filamentation region by a short argon cell. It results in ultrashort UV and VUV pulses at 270 and $162 \mathrm{~nm}$ with duration of $11 \mathrm{fs}$ as determined by cross correlation with the fundamental in a pump-probe setup with ionization detection; even shorter pulses are expected, if shorter fundamental pulses are employed. The path in argon was chosen short enough (18 mm), so as to avoid phase mismatch; hence, this setup should also allow for efficient sum-frequency generation (for example, adding tunable radiation to short fundamental pulses), which would be simpler in this case than in the previously demonstrated fourwave difference-frequency mixing experiments (e.g. [55, 56]). The conversion efficiency for the two harmonic yields is about $0.1 \%$ and $0.001 \%$ at Ar pressures of 200 and 50 mbar, respectively, for the TH and FH. (The lower yield of the FH is in part due to the four times lower pressure required to maintain phase-matching.) The pulse energy in both cases is sufficient for pump-probe experiments, even if there is no one-photon resonance (e.g., with Xe and Ar for cross correlation), and there is room for further optimization [5].

\subsection{Conclusions}

In this chapter methods for generation of light in the UV region were studied, namely supercontinuum (SC) and third- and fifth-harmonic generation. The SC radiation is made by selfphase modulation in self-focusing in argon (see also Chapter 1). All the important features of the generated UV are related to its use as the pump radiation in pump-probe experiments for time-resolved studies of chemical reactions; these are the tunability and the cut-off wavelength, the conversion efficiency, the short pulse duration and the pulse-to-pulse and pointing stability of the generated radiation. 
The spectrum of the SC extends continuously towards the UV region, having a large bandwidth and reaching down to $250 \mathrm{~nm}$, starting with fundamental pulses of 10 -fs duration, and even to $210 \mathrm{~nm}$ with 6-fs pulses. Limiting the input energy by an iris, so that only one filament is generated, results in an output beam with stable spectrum, energy, pulse shape and beam direction from pulse to pulse. The yield of the UV (about $0.1 \%$ of the input in a bandwidth of $9 \mathrm{~nm}$ ) proved to be sufficient for using it as the pump in time-resolved experiments $[3,4,8]$. Another remarkable observation, very useful for applications, is the absence of conical emission and the transverse homogeneity of the output spectrum with the 10-fs pulses. These features, in combination with the intrinsically short duration with which the UV is generated (it can be as short as the fundamental [4], if after generation any dispersive material is avoided), makes the supercontinuum radiation a very attractive source of light of tunable frequency, to be used in spectroscopic applications (an example was already demonstrated in $[8])$.

For this purpose extending the spectral range of the supercontinuum radiation further to shorter wavelengths is desirable and therefore understanding the underlying mechanisms responsible for its generation would be helpful. The spectral broadening turns out to depend on the input energy, pulse duration, interaction length, pressure and ionization of the nonlinear medium and the initial chirp; some questions need therefore to be addressed, such as the termination of the filament and the influence of the focal length or the unusual chirpdependence.

Finally, harmonic generation (at 270 and $162 \mathrm{~nm}$ ) proved efficient in terms of energy and pulse duration for using it in transient spectroscopy. The yields achieved were about $0.1 \%$ and $0.001 \%$ of the input for the third- and the fifth-harmonic radiation respectively and the pulse durations used in the pump-probe measurements were 30 and $10 \mathrm{fs}$ (without and with vacuum beam path, respectively) in the case of the TH and 11 fs in FH. From the results it is inferred that injection of two pulses, one of them short and the other of tunable wavelength, would give rise to tunable UV radiation by four-wave mixing. This would then be an alternative to the generation of an even broader SC and subsequently cutting out a narrow frequency range.

The wavelengths generated and used in the time-resolved measurements (Chapters 4, 5, 6) covered the UV region $270-340 \mathrm{~nm}$ (from the supercontinuum radiation) and the wavelengths 270 and $162 \mathrm{~nm}$ (from third- and fifth-harmonic generation). This radiation served as the pump pulse, which excites the molecules of interest and initiates the reaction under inves- 
tigation. Due to the shorter durations, the time resolution was much higher than previously, so that many more details and more insight into the dynamics were obtained. 


\section{3}

\section{Time-resolved spectroscopy}

\subsection{I ntroduction}

The so called pump-probe techniques enable us to monitor the real-time dynamics of a molecular system while undergoing a chemical reaction [57]; they have provided a tool with which one can actually follow and "see" the very microscopic changes in time (and in space with some probing methods) which take place in the system under consideration during the course of the reaction. In a pump-probe experiment a pump laser pulse prepares the system in an excited state and after a variable time delay a second laser pulse (probe) interacts with the molecule while this passes through a short-lived transition state. The fact that the interaction with the probe pulse depends on the instantaneous position of the molecule on the excited potential energy surface (PES) provides "snapshots" of the evolution of the system and therefore more information, than merely the "before" and "after" stages of the reaction or the relative quantum yields of its products, is now accessible (Fig. 3.1.1).

Many fundamental processes in matter evolve in the sub-picosecond time scale. For example many photochemical reactions are completed within some hundreds to some tens of femtoseconds ( $\left.1 \mathrm{fs}=10^{-15} \mathrm{~s}\right)$, e.g. the cis-trans isomerization of some organic molecules $[58,59]$. In the last years, femtosecond laser pulses have proven to be a very powerful tool for monitoring the dynamics of such ultrafast processes within the above mentioned pump-probe experiments, providing high time resolution, which the present work has extended down to 10-12 fs, also in the UV. 


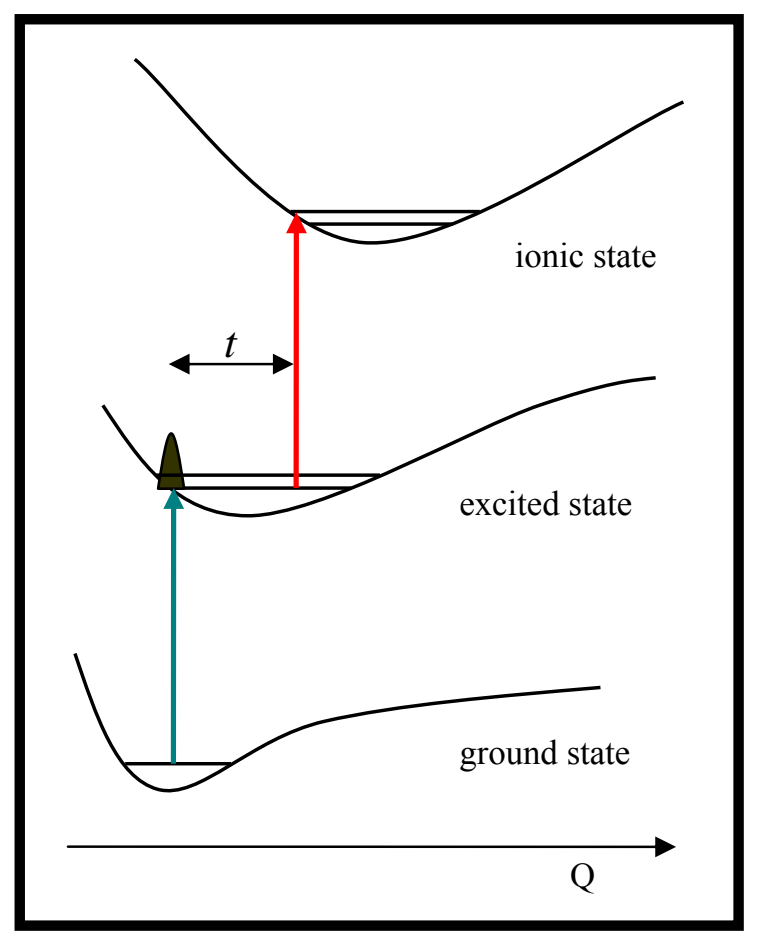

Fig. 3.1.1 Schematic illustration of the principle of a pump-probe experiment with ionization for probing. The pump pulse excites an electronic state and after variable delay $t$ the probe ionizes the excited neutral molecule.

\subsection{Pump-probe technique}

To monitor the reaction dynamics we use transient non-resonant multiphoton ionization as a probe. Initially a short $10-30$ fs UV laser (pump) pulse populates an excited state of the neutral molecule; owing to the broad spectrum of this pulse a number of vibronic states is coherently superimposed and a vibrational wave packet is created in this way. The prepared wave packet starts to propagate along the excited PES and the reaction under investigation is initiated. After a variable delay time $t$ the second (probe) pulse is applied to ionize the molecule (Fig. 3.1.1) and the ions are then detected with a time-of-flight mass spectrometer (TOFMS). The measured yields of the parent and fragment ions give several ion signals as a function of $t$ and provide information about the neutral molecule just before ionization occurs and hence about the evolution of the system determined by the details of the excited PES.

Other pump-probe experiments employ probing by e.g. the transient fluorescence or absorption [19]. Transient ionization, however, has the advantage that it gives rise to many ion signals that can differ in their temporal behaviour, thus revealing more details about the system under investigation than the above methods, where only one transient signal is usually recorded. Moreover, the redundant number of the independent ionic signals sometimes allows 
for an effective time resolution below the temporal width of the pump and the probe pulses as is explained in [60] (see also following sec. 3.4.3). Finally, a special advantage of ionization in particular of its non-resonant multiphoton variant - is that it doesn't depend on selection rules. Therefore also optically dark states or states whose absorption is not in an accessible wavelength range can be detected. Dark states play a crucial role in photochemistry (see next sections).

\subsection{Theoretical considerations of the molecular system}

\subsubsection{The Born Oppenheimer approximation}

The total energy $\hat{H}=\hat{T}_{\mathrm{nu}}+\hat{T}_{\mathrm{el}}+\hat{V}(\boldsymbol{r}, \boldsymbol{R})$ of a molecular system of nuclei $(n)$ and electrons (e) is the sum of their kinetic energy $\hat{T}_{\mathrm{nu}}(\boldsymbol{R})=-\frac{\hbar^{2}}{2} \sum_{n} \frac{1}{M_{n}} \nabla_{n}^{2}$ and $\hat{T}_{\mathrm{el}}(\boldsymbol{r})=-\frac{\hbar^{2}}{2} \sum_{e} \frac{1}{m_{\mathrm{el}}} \nabla_{e}^{2}$, respectively, and the potential energy $\hat{V}(\boldsymbol{r}, \boldsymbol{R})$ of the system, which depends on the electronic $\boldsymbol{r}$ and nuclear $\boldsymbol{R}$ coordinates.

To simplify the Schrödinger equation $\hat{H} \Psi(\boldsymbol{r}, \boldsymbol{R})=E \Psi(\boldsymbol{r}, \boldsymbol{R})$ for a molecule with wave function $\Psi(\boldsymbol{r} ; \boldsymbol{R})$ Born and Oppenheimer [61] considered that the nuclei are much slower than the electrons, due to their much larger mass. Since the latter can rapidly adjust to every newly acquired position of the former, Born and Oppenheimer (BO) treated the nuclear and electronic motion separately, independent of one another, approximating the molecular wave function as $\Psi(\boldsymbol{r}, \boldsymbol{R})=\mathrm{X}(\boldsymbol{R}) \Phi(\boldsymbol{r} ; \boldsymbol{R})$, where $\mathrm{X}(\boldsymbol{R})$ and $\Phi(\boldsymbol{r} ; \boldsymbol{R})$ are the nuclear and electronic wave functions respectively; $\mathrm{X}(\boldsymbol{R})$ depends only on the nuclear coordinates $\boldsymbol{R}$ while $\Phi(\boldsymbol{r} ; \boldsymbol{R})$ depends on the electronic coordinates $\boldsymbol{r}$ and only parametrically on $\boldsymbol{R}$. Using the method of the separation of variables and within the BO approximation (which mathematically is expressed as $\left.\nabla_{n}^{2} \Phi \mathrm{X}=\Phi \nabla_{n}^{2} \mathrm{X}+\mathrm{X} \nabla_{n}^{2} \Phi+2\left(\nabla_{n} \Phi\right)\left(\nabla_{n} \mathrm{X}\right) \cong \Phi \nabla_{n}^{2} \mathrm{X}\right)$, the Schrödinger equation becomes (see e.g. [62])

$$
\left[\hat{T}_{\mathrm{el}}(\boldsymbol{r})+\hat{T}_{\mathrm{nu}}(\boldsymbol{R})+\hat{V}(\boldsymbol{r}, \boldsymbol{R})\right] \Phi(\boldsymbol{r} ; \boldsymbol{R}) \mathrm{X}(\boldsymbol{R})=E \Phi(\boldsymbol{r} ; \boldsymbol{R}) \mathrm{X}(\boldsymbol{R}) \Rightarrow
$$




$$
\begin{aligned}
& \mathrm{X}(\boldsymbol{R}) \hat{T}_{\mathrm{el}}(\boldsymbol{r}) \Phi(\boldsymbol{r} ; \boldsymbol{R})+\Phi(\boldsymbol{r} ; \boldsymbol{R}) \hat{T}_{\mathrm{nu}}(\boldsymbol{R}) \mathrm{X}(\boldsymbol{R})+\hat{V}(\boldsymbol{r}, \boldsymbol{R}) \Phi(\boldsymbol{r} ; \boldsymbol{R}) \mathrm{X}(\boldsymbol{R})=E \Phi(\boldsymbol{r} ; \boldsymbol{R}) \mathrm{X}(\boldsymbol{R}) \Rightarrow \\
& \frac{1}{\Phi(\boldsymbol{r} ; \boldsymbol{R})} \hat{T}_{\mathrm{el}}(\boldsymbol{r}) \Phi(\boldsymbol{r} ; \boldsymbol{R})+\frac{1}{\mathrm{X}(\boldsymbol{R})} \hat{T}_{\mathrm{nu}}(\boldsymbol{R}) \mathrm{X}(\boldsymbol{R})+\hat{V}(\boldsymbol{r}, \boldsymbol{R})=E
\end{aligned}
$$

From the above Schrödinger equation two independent differential equations are obtained, namely

$$
\begin{aligned}
& {\left[\hat{T}_{\mathrm{el}}+\hat{V}(\boldsymbol{r}, \boldsymbol{R})\right] \Phi_{l}(\boldsymbol{r} ; \boldsymbol{R})=\varepsilon_{l}(\boldsymbol{R}) \Phi_{l}(\boldsymbol{r} ; \boldsymbol{R})} \\
& {\left[\hat{T}_{\mathrm{nu}}+\varepsilon_{l}(R)\right] \mathrm{X}(\boldsymbol{R})=E \mathrm{X}(\boldsymbol{R})}
\end{aligned}
$$

From Eq. 3.3.1.1 the eigenvalues of the electronic energy $\varepsilon_{l}$ for every different nuclear geometry are derived; $\varepsilon_{l}$ is a function of $f=3 N-6$ internal coordinates ( $3 N-5$ for a linear molecule) and constitutes an $f$-dimensional surface, the potential energy surface (PES).

Once the electronic energy as a function of $\boldsymbol{R}$ is known, it is usually assumed that the molecular vibrations are separable from one another, i.e. that the vibrational wave function is of the form $\mathrm{X}(\boldsymbol{R})=\prod_{k=1}^{f} \chi_{k}\left(\boldsymbol{R}_{k}\right)$, where $\chi_{k}$ denotes the vibrational wave function of the normal coordinate $\boldsymbol{R}_{k}$. This is in particular possible in the harmonic oscillator approximation. It simplifies solving Eq. 3.3.2 to obtain $\mathrm{X}(\boldsymbol{R}) ; \mathrm{X}(\boldsymbol{R})$ and Eq. 3.3.1.2 describe the vibrational motion of the nuclei in a potential $\varepsilon_{l}(\boldsymbol{R}) . f$ is the number of vibrational degrees of freedom. For an N-atomic molecule, $f=3 \mathrm{~N}-6(3 \mathrm{~N}-5$ if the molecule is linear).

$$
\text { If } \varepsilon_{l}(\boldsymbol{R}) \text { is expanded in a Taylor series as } \varepsilon_{l}(\boldsymbol{R})=\varepsilon_{l}\left(\boldsymbol{R}^{e}\right)+\left.\frac{1}{2} \sum_{k} \frac{d^{2} \varepsilon_{l}(\boldsymbol{R})}{d \boldsymbol{R}_{\boldsymbol{k}}^{2}}\right|_{\boldsymbol{R}^{e}}\left(\boldsymbol{R}_{k}-\boldsymbol{R}^{e}\right)^{2}
$$

[63] around the equilibrium geometry $\boldsymbol{R}^{e}$ and along the normal coordinate $\boldsymbol{R}_{k}$, truncating after the second term, the potential is that of an $f$-dimensional harmonic oscillator. Then, the solutions of Eq. 3.3.2 for $\chi_{k}\left(\boldsymbol{R}_{k}\right)$ are the Hermitian functions (containing the Hermitian polynomi- 
$\left.\operatorname{als\mathrm {H}_{\mathrm {v}_{k}}}\right) \chi_{k}^{\left(\mathrm{v}_{k}\right)}\left(\boldsymbol{R}_{k}\right)=\left(1 / \sqrt{2^{\mathrm{v}_{k}} \mathrm{v}_{k} !}\right) \exp \left(-\frac{1}{2} \gamma_{k} \boldsymbol{R}_{k}^{2}\right) \mathrm{H}_{\mathrm{v}_{k}}\left(\gamma_{k}^{\frac{1}{2}} \boldsymbol{R}_{k}\right)$ and $E_{k}=\left(\mathrm{v}_{k}+\frac{1}{2}\right) h v_{k}, \mathrm{v}_{k}=0$, $1,2 \ldots$, where $v_{k}$ is the frequency of the normal mode $k$ and $k=1 \ldots f$.

\subsubsection{Beyond the Born Oppenheimer approximation:} The conical intersection

Separating the nuclear from the electronic motion allows us to think of the heavy nuclei moving slowly over a potential energy surface representing the electronic energy; we speak then about adiabatic electronic potentials, energetically well separated. However, when the potential surfaces come close enough, the BO approximation breaks down because then the nuclei and the electrons move at similar speed, so that their motions couple and cannot be treated independently of each other. In these cases the operator $\hat{T}_{\mathrm{nu}}(\boldsymbol{R})=-\frac{\hbar^{2}}{2} \sum_{n} \frac{1}{M_{n}} \nabla_{n}^{2}$ of the nuclear kinetic energy operates not only on the nuclear wave function $\mathrm{X}(\boldsymbol{R})$ but also on the electronic wave function $\Phi(\boldsymbol{r} ; \boldsymbol{R})$ and terms proportional to $\left\langle\Phi_{j} \mid \nabla_{n} \Phi_{i}\right\rangle$ and $\left\langle\Phi_{j} \mid \nabla_{n}^{2} \Phi_{i}\right\rangle$ must be included in the nuclear Schrödinger equation (see corresponding terms in section 3.3.1), reflecting the fast changes of the adiabatic electronic states with the nuclear coordinates in the vicinity of a particular nuclear configuration. In other words, these "non-BO" terms indicate that changes of the electronic and nuclear coordinates $\boldsymbol{r}$ and $\boldsymbol{R}$ (i.e. the electronic and nuclear motion) take place within the same time scale and influence each other; the nuclei are now no longer thought of as confined to move periodically in one adiabatic surface and their motion evolves on more than one potential instead. This nonadiabatic interaction depends inversely on both the nuclear masses (the heavier the nuclei the more adequate the $\mathrm{BO}$ adiabatic approximation is for the description of the molecular system) and the energy gap between the surfaces [64]. This coupling of the nuclear and electronic motion is termed vibronic interaction.

An important example of the vibronic interaction is a conical intersection (CI) between different potential energy surfaces. A CI is formed when two $f$-dimensional electronic potentials touch (intersect) in some region of the nuclear configuration space, in the vicinity of which the vibronic coupling terms are very large, due to the energetic proximity. The CI space (in which the electronic states are degenerate) is of $f-2$ dimensions; in the remaining two di- 
mensions, which span the branching space, the degeneracy of the potential surfaces is lifted and the crossing is at least weakly avoided. Plotted versus the two coordinates of the branching space the electronic potentials in the vicinity of the CI appear to form a double cone (from which the name is derived) as illustrated in Fig. 3.3.2.1, where several characteristic examples of conical intersections are plotted in one coordinate.
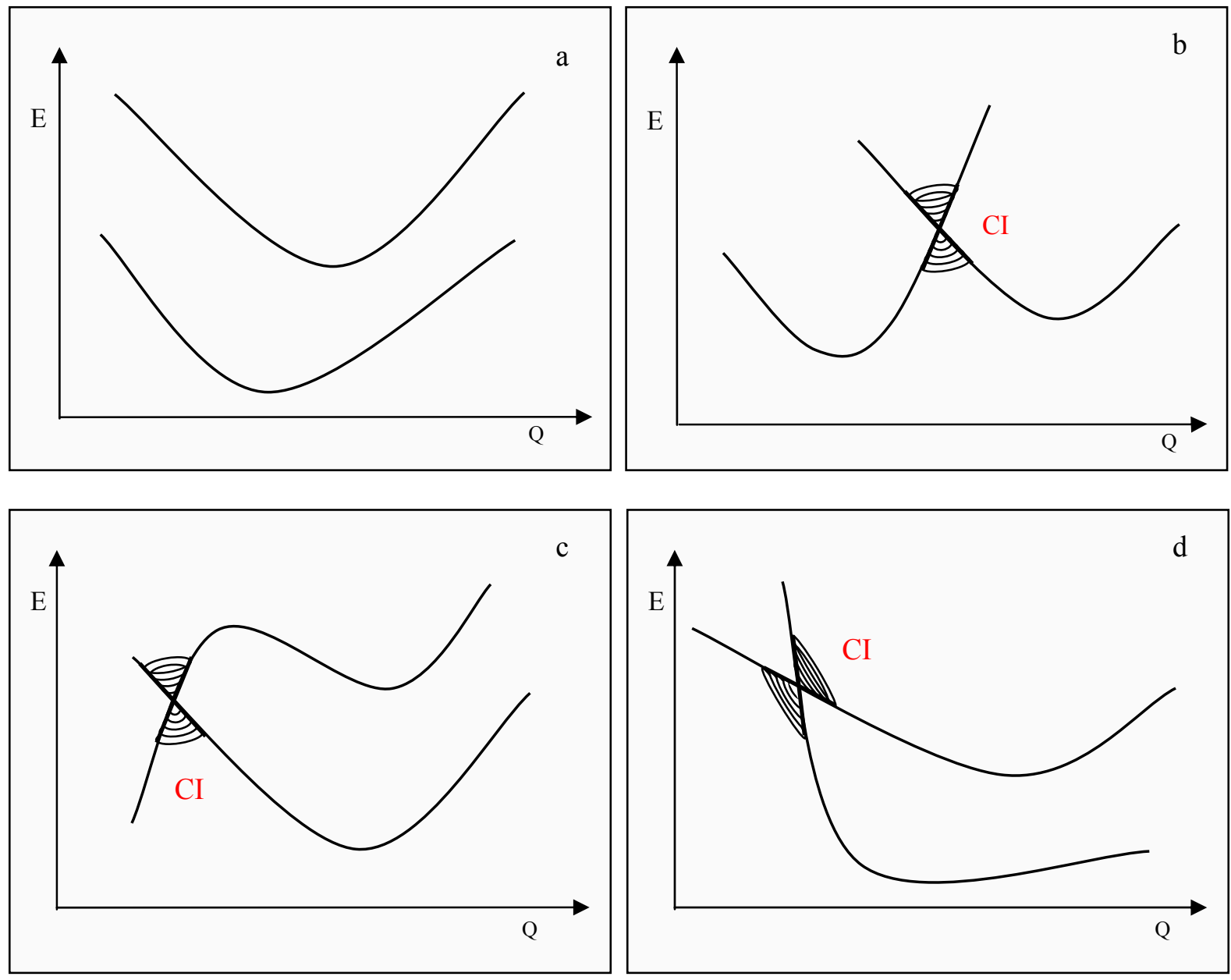

Fig. 3.3.2.1 (a) Two adiabatic potential surfaces, energetically well separated, plotted along the internal nuclear coordinate Q (b) Schematic representation of a conical intersection in one dimension (c) CI accessible via an energy barrier (d) A sloped CI

Fig. 3.3.2.1a depicts two energetically well separated adiabatic potentials; this is a very typical case for diatomic molecules, where the non-crossing rule is valid and potentials of the same symmetry type cannot intersect. In polyatomic molecules $(f \geq 3)$, however, there can be found in general some other coordinate (outside the drawing plane of Fig. 3.3.2.1a) along which the surfaces are allowed to cross at some point [65]. Fig. 3.3.2.1b and 3.3.2.1c show respectively an easily accessible CI (i.e. without activation energy from the upper branches of the potentials) and a CI accessible only via an energy barrier from the excited state minimum; 
in the former case the molecules can relax passing from the higher state, through the CI, to the lower energy minima very easily. Fig. 3.3.2.1d reflects the case where population can flow from the lower potential on the left-hand side down to the other around the lower cone of the $\mathrm{CI}$, with temporary deflection to the second coordinate of the branching space. Also the case of Fig. 3.3.2.1b provides a path around the lower cone, this time leading from minimum to minimum, requiring, however, activation energy.

Fig. 3.3.2.1 gives a good physical intuition for the role that CIs play in photophysics and photochemistry. The upper cone serves as a collection funnel; it collects population on the upper surface and guides it to the "outlet" (the tip of the cone) and from there to the lower surface. The intersection point, i.e. the ( $f-2)$-dimensional space, is no bottleneck $[66,67]$, because the wave packet can also cross over around the intersection point; the crossing needs a time less than a vibrational period $[66,67]$. The lower cone of the CI constitutes the branching region of the reaction path, where the molecules in fact decide to either return back to the state of the reactant (internal conversion) or to follow the channels towards the reaction products; the relative quantum yields are mainly determined here. The branching space is obviously crucial for the fate of the reaction, so that the CI plays a central role in photochemistry. The other way around, from the spectrum of the products one can sometimes infer the coordinates of the branching space (as demonstrated in Chapter 4 for ethylene).

For the two coordinates $\left(\boldsymbol{x}_{1}, \boldsymbol{x}_{2}\right)$ of the branching space one can choose $\boldsymbol{x}_{1}=\partial\left(\varepsilon_{1}-\varepsilon_{2}\right) / \partial \boldsymbol{R}_{k}$ and $\boldsymbol{x}_{2}=\left\langle\Phi_{1} \mid \partial \Phi_{2} / \partial \boldsymbol{R}_{k}\right\rangle$, where $\Phi_{1,2}$ are the adiabatic electronic wave functions of the two intersecting potential surfaces of energy $\varepsilon_{1,2}$ [68] and the integration is performed over all the electron coordinates. The vector of $x_{1}$ points to the direction along which the difference in the slopes of the two intersecting surfaces is the largest, while $x_{2}$ to the direction of nuclear displacement (from the cone point) that most strongly mixes the two adiabatic electronic wave functions (i.e. the direction of strongest vibronic interaction).

Symmetry often plays a crucial role in the description and classification of conical intersections $[64,65,69]$. Intersections can occur between nondegenerate electronic states of different or the same symmetry and also in cases where no symmetry is present at all; the former are called symmetry-allowed conical intersections. A very important class of CI are those arising from the Jahn-Teller (JT) effect. According to Jahn and Teller [70] if a nonlinear polya- 
tomic molecule of a symmetric geometry (e.g $\left.O_{\mathrm{h}}\right)$ is in a symmetry-induced degenerate electronic state (e.g. of $E$ or $T$ symmetry), there are always at least two non-totally symmetric (with respect to the symmetry of the molecule) normal coordinates along which the degeneracy is lifted; the two states energetically split and the symmetry of the system is broken. These two coordinates span the branching space of this "symmetry-induced" (JT induced) CI. The molecule in its new equilibrium configuration is of lower symmetry and energy than in the "original" symmetric structure, i.e. in the CI, which would be the energy minimum in the absence of any symmetry breaking. Other types of conical intersections are described in [64] and references therein.

To summarize, it should be now clear that conical intersections and their characteristic molecular geometry are of great importance for the dynamics of photo-induced chemical reactions of polyatomic molecules. As a matter of fact, in all ultrafast molecular processes (reactions and/or internal conversions), where a change of the electronic state is involved, the molecules pass through a CI during relaxation. The present work elucidates the role of CIs in the dynamics of several molecules in the gas phase.

\subsubsection{Selection rules in light absorption}

One-photon transitions of molecules from an initial electronic potential $\Phi_{1}$ and vibrational state $X_{1}^{(v)}(\boldsymbol{R})$ of quantum number v, to a higher potential $\Phi_{2}$ and vibrational state $X_{2}^{\left(v^{\prime}\right)}(\boldsymbol{R})$ of quantum number $\mathrm{v}^{\prime}$ are classified as symmetry-forbidden or -allowed according to whether the electric dipole moment $\mathrm{M}=-|e| \sum_{j} \boldsymbol{r}_{j}+|e| \sum_{i} Z_{i} \boldsymbol{R}_{i}$ of the transition vanishes or not; in the former case, the transition should normally have zero intensity. From the matrix element of the transition moment $\left\langle\Psi_{2}|\mathrm{M}| \Psi_{1}\right\rangle=-|e|\left\langle\Phi_{2}(\boldsymbol{r} ; \boldsymbol{R})\left|\sum_{j} \boldsymbol{r}_{j}\right| \Phi_{1}(\boldsymbol{r} ; \boldsymbol{R})\right\rangle\left\langle\mathrm{X}_{2}^{\left(\mathrm{v}^{\prime}\right)}(\boldsymbol{R}) \mid \mathrm{X}_{1}^{(\mathrm{v})}(\boldsymbol{R})\right\rangle[65$, 68] the symmetry selection rules that differentiate between allowed and forbidden transitions can be derived. For an allowed electronic transition the matrix element must be different from zero. When $\left\langle\mathrm{X}_{2}^{\left(\mathrm{v}^{\prime}\right)}(\boldsymbol{R}) \mid \mathrm{X}_{1}^{(\mathrm{v})}(\boldsymbol{R})\right\rangle \neq 0$ (i.e. when $\mathrm{X}_{1}^{(\mathrm{v})}$ and $\mathrm{X}_{2}^{\left(\mathrm{v}^{\prime}\right)}$ are of the same symmetry) this holds if the first integrand is a totally symmetric function or at least contains a totally symmetric component. In other words, the direct product of the symmetry species $\Gamma$ of $\Phi_{2}$, $\mathrm{M}$ and $\Phi_{1}$ must result in the totally symmetric representation $\Gamma_{1}$ of the point group of the 
molecule in order for $\left\langle\Psi_{2}|\mathrm{M}| \Psi_{1}\right\rangle$ to be different from zero, namely: $\Gamma\left(\Phi_{2}\right) \otimes \Gamma(\mathrm{M}) \otimes \Gamma\left(\Phi_{1}\right) \ni \Gamma_{1}$ or equivalently $\Gamma\left(\Phi_{2}\right) \otimes \Gamma\left(\Phi_{1}\right) \ni \Gamma(\mathrm{M})$. For transitions that are electronically forbidden but (weakly) allowed through vibronic coupling, the product $\Gamma\left(\Phi_{2}\right) \otimes \Gamma\left(\mathrm{X}_{2}^{\left(\mathrm{v}^{\prime}\right)}\right) \otimes \Gamma(\mathrm{M}) \otimes \Gamma\left(\Phi_{1}\right) \otimes \Gamma\left(\mathrm{X}_{1}^{(\mathrm{v})}\right)$ must be totally symmetric, or equivalently $\Gamma\left(\Phi_{2}\right) \otimes \Gamma(\mathrm{M}) \otimes \Gamma\left(\Phi_{1}\right)$ must be equal to $\Gamma\left(\mathrm{X}_{2}^{\left(\mathrm{v}^{\prime}\right)}\right) \otimes \Gamma\left(\mathrm{X}_{1}^{(\mathrm{v})}\right)$. The resulting selection rules in vibrational transitions within the forbidden electronic transitions are different from those occurring in an allowed electronic transition [65].

The square of the overlap integrals of the vibrational wave functions of the two electronic states $\left\langle\mathrm{X}_{2}^{\left(\mathrm{v}^{\prime}\right)} \mid \mathrm{X}_{1}^{(\mathrm{v})}\right\rangle$ constitute the so-called Franck-Condon factors, which determine the intensity of the individual vibrational components of the absorption band. These integrals reflect the Frank-Condon principle (FC principle) [71, 72], which states that on electronic excitation the heavy nuclei cannot follow the fast motion of the electrons during their interaction with the electric field and therefore the nuclear configuration $\boldsymbol{R}$ of the initial and final electronic states remains practically unchanged. Such "vertical" excitations from the ground vibrational level of the initial electronic state lead, in general, to the turning point of a higher vibrational (totally symmetric) state on the excited energy surface. However, along directions (normal coordinates) where the potentials are parallel the vibrational quantum number does not change; it nearly does not change if the potentials are not displaced (thus having coinciding minima) but possibly have different curvatures. The latter situation is typical for antisymmetric coordinates, in which case the FC factors are nonzero only for $\mathrm{v}^{\prime}{ }_{k}-\mathrm{v}_{k}=0,2,4 \ldots$ The Rydberg and ionic states of a molecule constitute an example of parallel potential energy surfaces (see the example in Chapter 4).

For two-photon, electronically allowed transitions, where only one virtual state $l_{n}$ (i.e. nonresonant intermediate state, most important if it lies between $\Psi_{1}$ and $\Psi_{2}$ ) is involved, the matrix element $\left\langle\Psi_{2}|\mathrm{M}| \Psi_{1}\right\rangle$ of the dipole transition moment consists of terms of the type $\left\langle\Phi_{2}(\boldsymbol{r} ; \boldsymbol{R})\left|\sum_{j} \boldsymbol{r}_{j}\right| l_{n}\right\rangle\left\langle I_{n}\left|\sum_{j} \boldsymbol{r}_{j}\right| \Phi_{1}(\boldsymbol{r} ; \boldsymbol{R})\right\rangle\left\langle\mathrm{X}_{2}^{\left(\mathrm{v}^{\prime}\right)}(\boldsymbol{R}) \mid \mathrm{X}_{1}^{(\mathrm{v})}(\boldsymbol{R})\right\rangle$ and the above symmetry product requires that $\Gamma\left(\Phi_{2}\right) \otimes \Gamma(\mathrm{M}) \otimes \Gamma\left(l_{n}\right) \ni \Gamma_{1}, \quad \Gamma\left(l_{n}\right) \otimes \Gamma(\mathrm{M}) \otimes \Gamma\left(\Phi_{1}\right) \ni \Gamma_{1}$. That is, both transi- 
tions $\left|\Phi_{1}\right\rangle \rightarrow\left|l_{n}\right\rangle$ and $\left|l_{n}\right\rangle \rightarrow\left|\Phi_{2}\right\rangle$ must be allowed. To generalize for multi-photon nonresonant excitations, all the individual steps in the sequence $\left|\Phi_{1}\right\rangle \rightarrow\left|l_{1}\right\rangle \rightarrow \ldots \rightarrow\left|l_{n}\right\rangle \rightarrow\left|\Phi_{2}\right\rangle$ would have to be allowed. The assumption that no real intermediate states are populated implies a vertical excitation, which is again governed by the FC integral $\left|\left\langle X_{2}^{\left(v^{\prime}\right)} \mid X_{1}^{(v)}\right\rangle\right|^{2}$. This is the typical case for our probe transitions.

Finally, the spin-selection rule imposes that the two electronic states involved in the transition are of the same multiplicity. This results from the fact that when the electron spin wave function $\sigma$ is also taken into account in the total molecular wave function $\Psi(\boldsymbol{r}, \boldsymbol{R})=\mathrm{X}(\boldsymbol{R}) \Phi(\boldsymbol{r} ; \boldsymbol{R}) \sigma$ the transition moment matrix element acquires then an additional factor $\left\langle\sigma_{2} \mid \sigma_{1}\right\rangle$, which is nonzero only in the case where the two electronic states have the same spin. The transition is then called spin-allowed. However, spin-forbidden transitions do occur; these kinds of transitions are induced by spin-orbit coupling, which mixes states of different multiplicity [65]. Spin-forbidden transitions are in general much weaker than allowed transitions. They are postulated in the literature for transition metal complexes for instance; however it is shown in Chapter 6 that they play no role in chromium hexacarbonyl and homologues.

\subsection{Reaction dynamics}

\subsubsection{Deactivation processes}

In the traditional description, photophysical processes were considered separately from photochemical processes, whereas in a more modern view (sec. 3.4 .2 below) internal conversion at least if it is ultrafast - has the initial part of the path in common with a reaction. The paths branch at a CI. Within the previous description of the photophysics, the energy taken up upon absorption of light by a molecule can be dissipated either through unimolecular processes, in the form of emitted radiation or by radiationless transitions, or through bimolecular processes when energy is transferred to other molecules, e.g. through collisions in liquids. The latter take typically $5-10 \mathrm{ps}$, but play no role in the gas phase on an ultrafast time scale. Two important mechanisms of radiationless deactivation are the internal conversion (IC) and intersystem crossing (ISC); the former concerns transitions between isoenergetic levels of different electronic states of the same multiplicity, whereas the latter transitions between states of dif- 
ferent multiplicity. The Fermi golden rule for the dynamics of transitions between two electronic states determines the rate constants of these radiationless decays [68], considering the energy gap between their electronic potentials, as well as the density of viabrational levels lying within them at a given energy. It predicts that the IC rate can reach up to $k_{\mathrm{IC}}=10^{12}-$ $10^{14} \mathrm{~s}^{-1}$ for $S_{n} \rightarrow S_{1}$ transitions, but be as small as $k_{\mathrm{IC}}<10^{8} \mathrm{~s}^{-1}$ for the $S_{1} \rightarrow S_{0}$ transitions; the rate of ISC is $k_{\mathrm{ISC}}=10^{6}-10^{11} \mathrm{~s}^{-1}$ and $k_{\mathrm{ISC}}=10^{4}-10^{-1} \mathrm{~s}^{-1}$, depending on whether the relaxation starts from a singlet or a triplet state. Owing to fast radiationless deactivation from the excited state (the pure radiative lifetime is of the order of tens of nanoseconds), luminescence is hardly observed in many cases such as e.g. butadiene and hexatriene [73-75].

Even in the gas phase, where no collisions between molecules take place (collision rate $\approx 10^{10} \mathrm{~s}^{-1}$ bar $^{-1}$ ), many photo-induced chemical reactions are more and more recognized to proceed through several radiationless transitions between the electronic potentials. Mediated very often by the presence of real surface crossings (CIs), some relaxation processes are found to be completed within some tens of femtoseconds, such as in the case of the photo-dissociation of some transition metal complexes (Chapter 6) or the internal conversion and isomerization of ethylene (Chapter 4).

Investigating the actual intermediate changes of the molecule during the course of a reaction (as opposed to only observing the reactants and the products in their equilibrium geometries) constitutes the main goal of any study of the nuclear dynamics of a chemical reaction.

\subsubsection{The pathway approach}

Fuß et al. [76] use a "pathway approach" for the description of the dynamics of ultrafast photophysical and photochemical processes that involve several PESs after initial preparation of the system to an excited state; they trace the pathway of the molecules along potentials considering local properties such as surface slopes, energy barriers, saddle points and intermediate states and finally conical intersections (CI). Such intersections are found in special regions of the $f$-dimensional space of the molecular potentials (sec. 3.3.2). Easily accessible conical intersections, playing the role of a collection funnel, provide continuous paths between electronic surfaces and can therefore explain some very short lifetimes (in the femtosecond time scale) and ultrafast internal conversions. Furthermore, the CI constitutes the branching point between the recovery of the educt (internal conversion) and the formation of one or several product channels. That is, photophysics and photochemistry have the initial path in common. 
An important role for the dynamics and the final outcome of a reaction is played by the socalled dark states. The term "dark states" refers to states that are spectroscopically not accessible by one-photon, vertical excitation from the ground state of the molecule. An important class of such states are regions on the PES that are far displaced from the equilibrium geometry of the reactant ground state, e.g. half-way to the product. The "pericyclic minimum" on the 2A two- electron excited surface, which is involved in the photochemical pericyclic reactions (like the ring opening of many cyclic organic molecules, e.g. cyclohexadiene, Chapter 5), constitutes a very characteristic example of a dark state. A similar case is the "perpendicular minimum" in cis-trans isomerisation of CC double bonds (e.g. ethylene, Chapter 4).

Many photochemical reactions such as pericyclic rearrangements of organic molecules, isomerizations of linear polyenes and dissociations have been investigated experimentally and interpreted within the pathway approach, in terms of the "population flow" along potential surfaces and through CIs, down to the ground state of the products or the reactant. This is the approach used in the present work as well.

\subsubsection{Description of the dynamics by rate equations and periodic modulations}

In the absence of coherent effects, the flow of population along the PES can be described by rate equations [77]. For example, if there is no branching, the population flows from one location to another on the PES, $\mathrm{L}_{i} \rightarrow \mathrm{L}_{i+1}$ with a time constant $\tau_{i}\left(\operatorname{rate} \tau_{i}^{-1}\right)$. The rate equations predict for the time-dependent populations of $\mathrm{L}_{i}$ a sum of exponentials, e.g. a single exponential for $\mathrm{L}_{1}$ and two exponentials for $\mathrm{L}_{2}$ (which reflect the rising and decaying times of the population in this window) etc.

Each location has its own mass spectrum. Conversely, each ion signal usually contains contributions from more than one $\mathrm{L}_{i}$. Hence, the time behaviour of the ion of mass $m$ is represented by a linear combination of the time-dependent populations (i.e. sum of exponentials) of every observation window $\mathrm{L}_{i}$ on the PES, the coefficients being the probabilities ${ }^{m} \sigma_{i}^{(l)}$ of the excited neutral to produce this ion from the location $\mathrm{L}_{i}$ by absorbing $l$ probe photons. In other words, the ion signal is simulated by a sum of exponentials convoluted with the instrumental function, i.e. the cross-correlation function between the UV-excitation pulse and the lth power of the probe-ionizing pulse, which is of the form (see Appendix for derivation) 


$$
\operatorname{Sig}(\Delta t)=\sum_{n} \alpha_{n} \times \exp \left(-k_{n} \Delta t\right) \times\left(1+\operatorname{erf}\left[\frac{1}{\sqrt{2}}\left(\Delta t \gamma-k_{n} / 2 \gamma\right)\right]\right)
$$

where each $\alpha_{n}$ is a linear combination of the cross-sections ${ }^{m} \sigma_{i}^{(l)}, \gamma^{-1}=\sqrt{l^{-1} \tau_{\mathrm{pr}}^{2}+\tau_{\mathrm{pu}}^{2}}, \tau_{\mathrm{pu}}$ and $\tau_{\mathrm{pr}}$ are the Gaussian widths of the pump and the probe pulse. The cross-sections and the time constants $\tau_{i}=k_{i}^{-1}$ are then the outcome of this fitting procedure. The number of probe photons $l$, required for ionization in principle also depends on $\mathrm{L}_{i}$; however, as can be easily confirmed by the relation for the instrumental function, its width $\gamma^{-1}$ is nearly independent of $l$, if $l \geq 3$, that is for almost all cases. It should be noted that the locations $\mathrm{L}_{i}$ to be identified are more like extended regions (indicated by arrows in the figures of the next sections) on the potential surfaces rather than discrete points; the borders between them result from properties of the probe process, the change of ionization probability and fragmentation pattern, the changes of which can be relatively continuous and not abrupt and therefore distinct. The "lifetimes" (of the populations within these $\mathrm{L}_{i}$ ) are indicated besides the arrows, if the traveling times of the wave packet through these locations are expected in the same range.

The temporal halfwidth $\gamma^{-1}$ of the instrumental function (correlation function of the pump pulse with a high power $l$ of the probe pulse) represents the time resolution, which is needed to separate two short processes from each other. However, with sufficient signal-to-noise ratio, deconvolution with the instrumental function (which must be known) can in principle provide a single time constant, even if it is much shorter than $\gamma^{-1}$. This can be easily understood if the simplest case of each observation window giving rise to only one ion signal is considered (that is every signal of mass $m$ exhibiting only one time constant). For example, in the case of $\mathrm{Cr}(\mathrm{CO})_{6}{ }^{+}$(Chapter 6) a single exponential decay with time constant of 12.5 fs was determined and from $\mathrm{Cr}(\mathrm{CO})_{5}{ }^{+}$another time constant of $18 \mathrm{fs}$, both below the 30-fs time resolution; it would not be possible to extract two time constants, $<30$ fs each, from one signal. But it is possible from two signals, if the $\mathrm{L}_{i}$ give rise to different ion masses $m$ or if the signals are at least linearly independent (in the linear combination of exponentials). In general, every location $\mathrm{L}_{i}$ has a different mass spectrum, so that several short time constants can be extracted from the above described curve fitting. This is a great advantage of having several signals, as it is usually the case in transient mass spectroscopy. 
Often, a time constant $\tau$ is derived by evaluating the signal in the time range about $5-8 \times \tau$ (with simple exponential fit without convolution with the instrumental function) before using it in the full fitting curve to extract additional information (if the signal-to-noise ratio is sufficient and if the pulse shapes are well represented by Gaussians over three orders of magnitude). While the signal is still exponential in this time range, the Gaussian instrumental function has already faded away [34, 60]. In this way, evaluation is done outside the pump-probe overlapping time and so complications that can arise due to the high probe intensity during the pump-probe overlap (e.g. near the signal maximum) and influence the temporal signal are avoided (such as, for instance, exciting a higher initial state than the desired one by absorption of one pump and additional probe photons).

Sometimes coherent oscillations are superimposed on the exponential time functions. They are interpreted as a vibration of the wave packet within a given location $\mathrm{L}_{i}$; if the ionization probability ${ }^{m} \sigma_{i}^{(l)}$ depends on the vibrational coordinate, then this quantity is periodically modulated leading in addition to a corresponding signal modulation. This means that it is not the population flow that shows the periodicity; this is still represented by the exponential part.

To account mathematically for the vibrations of the molecule, the coefficients ${ }^{m} \sigma_{i}^{(l)}$ are modulated in time by one or more periodic terms of the form $\left[1+A \exp \left(-t / \tau_{\text {deph }}\right) \cos \left(2 \pi t / T_{\text {osc }}-\varphi\right)\right]$, where $T_{\text {osc }}$ is the oscillation period (frequency c $v$ ), $\varphi$ a phase shift, $\tau_{\text {deph }}$ the pure dephasing time and $A$ the amplitude. The cross-section for absorption of $l$ probe photons then becomes ${ }^{m} \sigma_{i}^{(l)}={ }^{m} \sigma_{i}^{(l)}{ }_{0}\left[1+A \exp \left(-t / \tau_{\text {deph }}\right) \cos \left(2 \pi t / T_{\text {osc }}-\varphi\right)\right]$, with more factors like the square bracket when necessary.

The considerations above imply that two processes below time resolution can be detected if they belong to different $\mathrm{L}_{i}$. However, high time resolution is required to reveal an oscillation of high frequency (short period), because it takes place in one $\mathrm{L}_{i}$ of the excited molecule. Besides time resolution, detecting such oscillations, furthermore, requires that the upper and lower potentials of the probe transition be shifted against each other or differ in curvature, so that the distance between them (i.e. the ionization energy) depends on the vibrational coordinate, so that the cross-section for ionization (ionization probability) and hence the signal is modulated with the frequency of the corresponding vibration. Additionally, some vibrations and, therefore, the amplitude of modulation of the ion signal may be enhanced by some inter- 
mediate resonances in the neutral molecule or in the ion, whereas others are not [78]. On the other hand, as is explained in [79], cases in which an oscillation might not be observed in the pump-probe experiment (although it might be excited and have a frequency low enough compared to the time resolution), are, for example, when the oscillation is covered by a stronger one and/or by unfortunate frequency coincidences. Other reasons for not detecting an oscillation are a rapid loss of coherence (too short dephasing time) or even complete absence of coherence. This can result from the slightly different trajectories, which the wave packets of different molecules might follow along the potentials and which would lead to (slightly) different vibrational frequencies, so that they are not well enough in phase; or perhaps from changes in the direction of motion of the wave packets or other reasons. One should therefore not try to conclude much from the absence of an expected oscillation. But if an oscillation is indeed detected and if its vibrational coordinate can be assigned, it is a source of valuable information on the potentials and their slopes.

Finally, we consider the ways that fragmentation in the pump-probe experiments takes place. In most of the investigated molecules, there is no dissociation, at least not within an ultrashort time in the range of 10 to $30 \mathrm{fs}$. Hence, the fragment ions observed must have been formed after ionization. We assume the following mechanisms of ionic dissociation:

1) On the way down the potentials of the neutral molecule, relaxation converts electronic to kinetic energy. Most of this excess vibrational energy is transferred upon vertical ionization to the ionized molecule. ("A hot neutral gives rise to a hot ion".) If this energy exceeds the threshold for fragmentation of the ion, then only lighter fragments will be observed instead of the parent ion. Consequently the fragmentation pattern depends on the kinetic excess energy or the electronic energy (their sum is constant) of the neutral species on the PES. Therefore the energy of the location can be estimated.

2) The excess electronic energy with which the ion is produced. This happens when the molecule relaxes along a descending excited-state surface, while the ionic ground-state surface rises along the same direction. (More probe photons are needed in this case than in the Franck-Condon region, so that the ionization probability will go down.) If again this amount of energy exceeds the fragmentation threshold of the ion, lighter ion fragments will be generated. 
3) Photochemistry of the ion. This requires further absorption of probe photons by the parent ion, sometimes involving intermediate resonant states. These resonances depend on the geometry of the ion and therefore on that of the neutral, assuming a vertical ionization mechanism.

The above considerations of the population flow and fragmentation are summarized in Fig. 3.4.3.1.

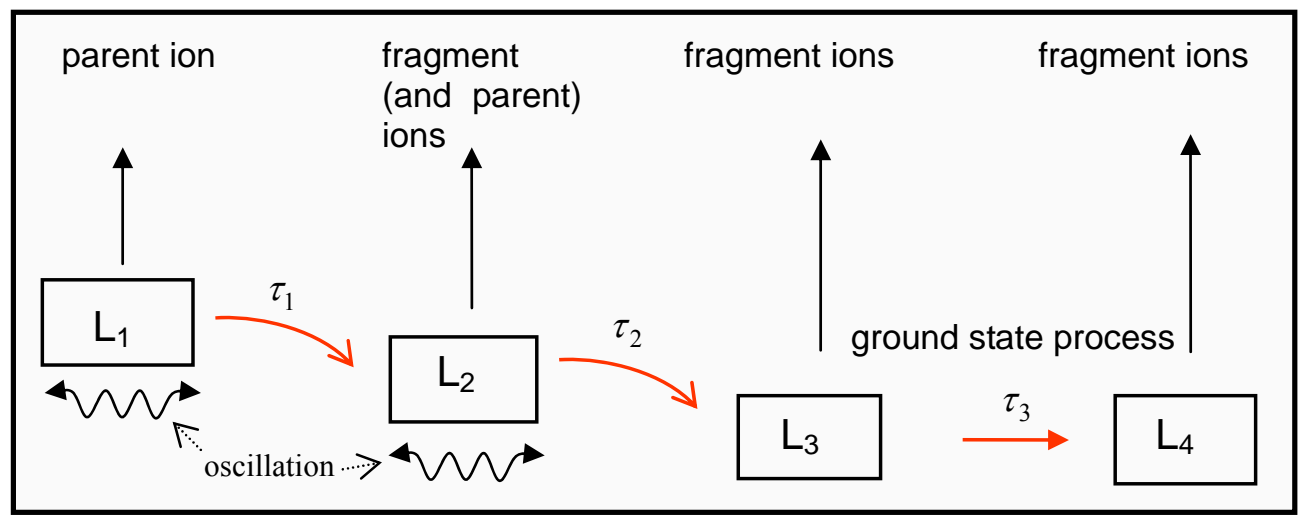

Fig. 3.4.3.1 Typical scheme for producing parent and fragment ions in the pump-probe experiment. After excitation of the location $\mathrm{L}_{1}$ by absorption of one pump photon the probe pulse arrives with delay $t$ and ionizes the molecules as they relax down to the ground state through the several locations $\mathrm{L}_{i}$ on the PES. The fragmentation pattern changes as electronic relaxation proceeds, with the parent ion being produced mainly in $\mathrm{L}_{1}$; ionization from the later windows leads to dissociation of the parent ion and observation of only ionic fragments. Two oscillations (vibrations in $\mathrm{L}_{1}$ and $\mathrm{L}_{2}$ ) are also indicated.

To point out once more: fragmentation by the femtosecond pulses used in these experiments typically takes place after ionization, that is, after the generation of the energetically hot parent ion and not in the neutral molecule [80]. This is because the neutral molecule does not have enough time to dissociate to lighter fragments during delay times $\leq$ ca. $1 \mathrm{ps}$, whereas the molecular ion has plenty of time (some nanoseconds) for dissociation during the acceleration time in the ion source $(\sim 100 \mathrm{~ns}$ for mass $100 \mathrm{u})$. 


\section{4}

\section{Dynamics of internal conversion and isomerization}

\section{of ethylene and ethylene- $d_{4}$}

\subsection{Experimental}

The molecular system and the reaction investigated here are ethylene and ethylene- $d_{4}$ excited in the maximum of its first UV transition, at $162 \mathrm{~nm}$, and its subsequent relaxation path along the potentials including also isomerization about the double bond. This is a fundamental problem of photochemistry and is an active field of theoretical research. It is the main reason why we developed a laser providing short vacuum UV pulses. The laser system and the experimental set-up used to generate the ultrashort 810 -nm probe and the VUV pump pulses for the time-resolved studies were described in Chapter 2. The 162-nm, 11-fs radiation is the fifthharmonic of the 810-nm fundamental pulses and has an energy of $4 \mathrm{~nJ}$, corresponding to an intensity of about $10^{10} \mathrm{~W} \mathrm{~cm}^{-2}$ in the focusing region, which is in the ionization chamber of the time-of-flight mass-spectrometer (TOFMS). The probe pulses at $810 \mathrm{~nm}$ have an energy of about $3 \mu \mathrm{J}$ and a duration of 12 fs (intensity in the focus of about $3 \times 10^{13} \mathrm{~W} \mathrm{~cm}^{-2}$ ); for some measurements raising the probe intensity by a factor of 3 was necessary (intensity of $\approx 10^{14} \mathrm{~W}$ $\left.\mathrm{cm}^{-2}\right)$.

The pump and probe beams are combined in the TOFMS (Chapter 2, section 2.5, Fig. 2.5.1) and the transient ions (parent and fragment ions) are detected versus delay time (varied in steps of $3.3 \mathrm{fs}$ ) between the two pulses. In every set of measurements Xe is introduced at a pressure of abound $10^{-5}$ mbar in the TOFMS and $\mathrm{Xe}^{+}$is recorded in parallel with the other signals, using boxcar integrators. In the case of ethylene and the 162-nm pump pulses, Xe is ionized by absorption of one VUV photon and three IR photons (ionization energy $12.15 \mathrm{eV}$ ). 
Since no process of finite lifetime is involved in the dynamics of its ionization, the $\mathrm{Xe}^{+}$signal corresponds to the cross correlation function of the pump and the third power of the probe pulses, so that the VUV pulse duration can be determined for every set of measurements (the duration of the IR pulses is measured by a commercial autocorrelator (Femtolasers)). Furthermore, the time zero can also be determined from $\mathrm{Xe}^{+}$, that is the time when the pump and the probe pulses perfectly overlap in time; negative times correspond to the probe pulses arriving first at the target and the other way around for positive delay times (for more details see Chapter 2).

The pressures of the gases in the ionization region of the TOFMS are between $10^{-7}$ and $10^{-4}$ mbar, the latter for the weakest signals. The absorption cross-section of ethylene at $162 \mathrm{~nm}$ $\left(\sigma \approx 3 \times 10^{-17} \mathrm{~cm}^{2}\right.$, Fig. 4.2.2) implies a saturation energy density of $h v / \sigma=40 \mathrm{~mJ} \mathrm{~cm}^{-2}$. In the focusing region of the TOFMS the estimated VUV beam cross-section is about $3 \times 10^{-3} \mathrm{~mm}^{2}$ and the corresponding energy density is about $0.13 \mathrm{~mJ} \mathrm{~cm}^{-2}$, i.e. $0.3 \%$ of the saturation energy density; therefore $0.3 \%$ of the ethylene molecules are excited. This energy and percentage of excited molecules was even sufficient to detect very weak signals such as those originating from molecules after their return to the ground state; for ionization from there, five more probe photons are required than from the Franck-Condon region (from where 1 VUV + 2 IR photons are required; ionization energy $10.51 \mathrm{eV}$ ). Previous experiments [81-84] did not detect such signals.

\subsection{Background}

Ethylene is the simplest unsaturated hydrocarbon. It has a double bond between the two carbon atoms, a planar geometry in its ground state $\mathrm{S}_{0}$ (Fig. 4.2.1) and belongs to the $D_{2 \mathrm{~h}}$ symmetry point group.

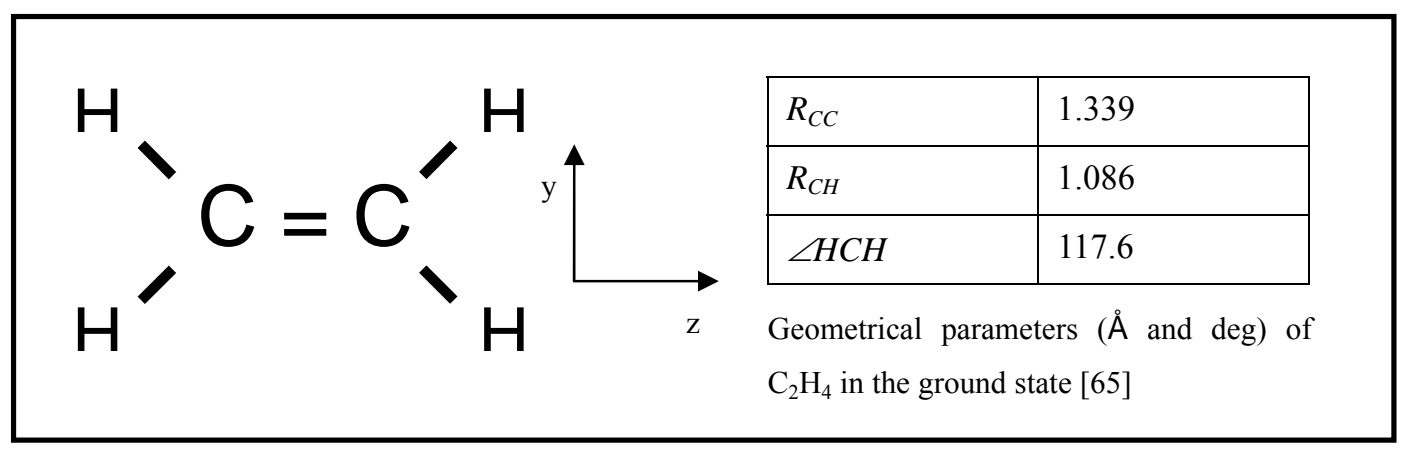

Fig. 4.2.1 Ethylene molecule. 
Its molecular orbitals (MOs) are constructed by the two $2 s$ and six $2 p\left(p_{x}, p_{y}, p_{z}\right)$ carbon atomic orbitals and the four $1 s$ hydrogen atomic orbitals $[62,85]$. The highest occupied molecular orbital (HOMO) is the $\pi$ orbital (symmetry $b_{3 \mathrm{u}}$ ), whereas the lowest unoccupied molecular orbital (LUMO) is the $\pi^{*}\left(b_{2 \mathrm{~g}}\right)$, both formed by the two $2 p_{x}$ carbon atomic orbitals. The ground state $1{ }^{1} \mathrm{~A}_{\mathrm{g}}$ corresponds to the electron configuration:

$$
(\text { core })^{4}\left(2 a_{g}\right)^{2}\left(2 b_{1 u}\right)^{2}\left(1 b_{2 u}\right)^{2}\left(3 a_{g}\right)^{2}\left(1 b_{3 g}\right)^{2}\left(1 b_{3 u}\right)^{2}
$$

Fig. 4.2.2 shows the absorption spectra of ethylene and ethylene- $d_{4}$ adapted from [85]. The $\mathrm{C}_{2} \mathrm{H}_{4}$ spectrum $[85,86]$ shows a broad absorption band in the vacuum UV region, at $150-$ $200 \mathrm{~nm}$; it consists of a long progression of bands separated by approximately $800 \mathrm{~cm}^{-1}$, which, towards shorter wavelengths, is believed [85] to continue as a continuum (dotted line in Fig. 4.2.2). It is assigned to the $\pi \rightarrow \pi^{*}$ transition. Its maximum is at $162 \mathrm{~nm}(7.65 \mathrm{eV})$, where it is superimposed with the relatively sharp $\pi \rightarrow 3 s$ Rydberg band. The vibrational structure observed in the long wavelength region of the spectrum is now assigned to a progression due to the torsional mode. This was experimentally established by the deuterium effect [87] and by the resonance Raman spectrum $[88,89]$.

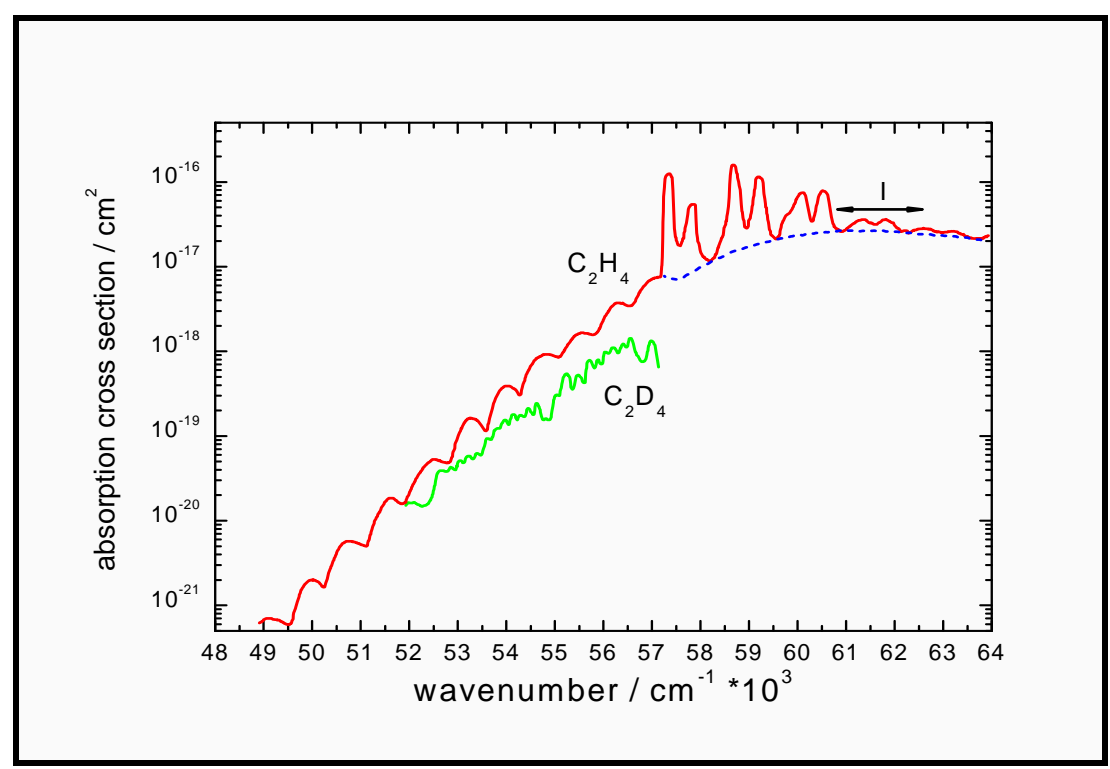

Fig. 4.2.2 UV spectra of ethylene and ethylene- $d_{4}$ (adapted from $[85,90]$ ), with the wavelength and bandwidth of the excitation laser also indicated. The dotted line is the estimation of $[85,90]$ for the $\pi \pi^{*}$ background below the Rydberg transition. See also the spectra shown in $[89,91]$. 
The first excited singlet state $\mathrm{S}_{1}$ (the ${ }^{1}\left(\pi \pi^{*}\right)$ state of $B_{1 \mathrm{u}}$ symmetry) is also called V ("valence") state, while $\mathrm{Z}$ is the doubly excited $\left(\pi^{*^{2}}\right)$ zwitterionic state of $A_{\mathrm{g}}$ symmetry. Their vertical excitation energies in planar ethylene are $7.65 \mathrm{eV}(162 \mathrm{~nm}$, Fig. 4.2.2) and $13.1 \mathrm{eV}$ (estimated) respectively, while both states have their minima at the $90^{\circ}$-twisted geometry, at an energy about $2 \mathrm{eV}$ lower than the Franck-Condon region of the $\pi \pi^{*}$ state [87], with the $\mathrm{Z}$ potential being slightly lower than V [68]. Finally, the barrier for torsion to $180^{\circ}$ in the ground state $\left(\mathrm{S}_{0}\right)$ is calculated around $3.0 \mathrm{eV}[92,93]$, so that the $\mathrm{S}_{1}-\mathrm{S}_{0}$ energy gap at $90^{\circ}$ is about 2.6 $\mathrm{eV}$.

The structure of ethylene in the lowest Rydberg state $\left(\pi 3 s,{ }^{1} \mathrm{~B}_{3 \mathrm{u}}\right)$ is also different from that in $\mathrm{S}_{0}$. The molecule has been shown to have a slightly twisted geometry in this state with minimum at a dihedral angle of $25^{\circ}-30^{\circ}$ and a torsional barrier of $290 \mathrm{~cm}^{-1}$ at $0^{\circ}$ [94, 95]; beyond the minimum the $\pi 3 s$ Rydberg potential rises again reaching a conical intersection (a degenerate state) at $90^{\circ}$ (Fig 4.2.3). The low barrier results in tunnelling and the effective symmetry of the molecule remains $D_{2 \mathrm{~h}}$. The energy of the $\pi 3 s$ Rydberg state at the planar configuration is about $7.2 \mathrm{eV}$ [85]. According to Fig. 4.2.2, the $\mathrm{V}$ and the $\pi 3 s$ Rydberg states are both excited at $162 \mathrm{~nm}$ with the Rydberg transition having about 5-10 times less probability; the two potentials intersect at a torsional angle of $\approx 35^{\circ}$ [92]. Fig. 4.2 .3 shows the potentials of the ground and the lowest excited electronic states of ethylene along the torsional angle, i.e. the angle between the two planes of the two methylene groups. The arrows indicate the expected relaxation paths from the excited states.

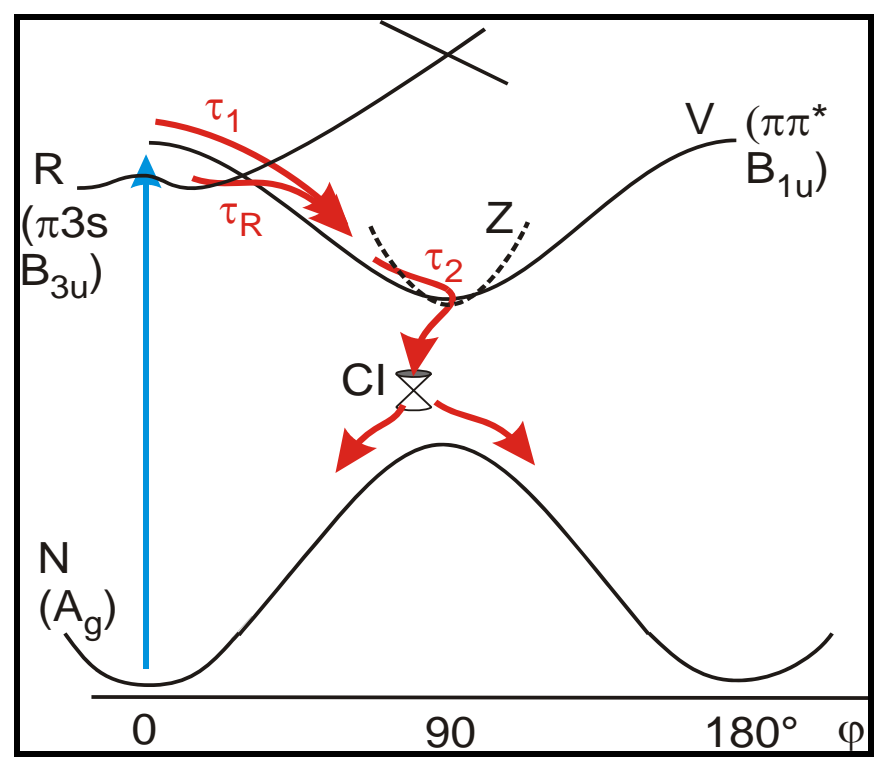


Fig. 4.2.3 The ground (N), first excited (V), zwitterionic (Z) and the $\pi 3$ s Rydberg states of ethylene; the initial reaction path of the relaxation back to the ground state after excitation at $162 \mathrm{~nm}$ is also shown (see below).

The vertical ionization energy of ethylene is $10.51 \mathrm{eV}$. Photoionization by removal of one electron from the $\pi$ orbital leads to the ground ${ }^{2} \mathrm{~B}_{3 u}$ state of $\mathrm{C}_{2} \mathrm{H}_{4}^{+}$; the ethylene cation in its equilibrium geometry is twisted away from planarity and has $D_{2}$ symmetry, with its ground state being of similar geometry as the $\pi 3 s$ Rydberg state of ethylene. The twisted dihedral angle and torsional barrier have been determined to be about $29^{\circ}$ and $357 \mathrm{~cm}^{-1}$ respectively $[94,96]$.

The ethylene structure, spectra, potentials and reaction dynamics have been extensively studied both theoretically and experimentally, since its geometrical changes and cis-trans isomerization through breaking of the $\mathrm{C}=\mathrm{C} \pi$ bond is a common feature for many photoinduced reactions in organic photochemistry. Upon excitation in the V state by absorption of one VUV photon at $162 \mathrm{~nm}, \mathrm{C}_{2} \mathrm{H}_{4}$ is known to undergo radiationless relaxation to the ground state (internal conversion) or cis-trans isomerize (which is detectable in isotopically labelled ethylene). In fact, the "perpendicular minimum" of the Z state (Fig. 4.2.3) turns out to be a saddle point, when additional modes besides torsion are considered; along the pyramidalization [93, $97,98]$ and/or the $\mathrm{H}$ migration coordinate $[92,99,100]$ the energy is lowered further and the molecule can reach a conical intersection with the ground state. If this path proceeds without any energy barrier, the ultrashort excited state lifetime of ethylene found in this work could be explained. Apart from isomerization, other reactions induced by UV excitation are the elimination of atomic and molecular hydrogen $[101,102]$; they are nowadays considered to proceed in the hot ground state arising from internal conversion (see, e.g., [103]).

\subsection{Results and analysis}

The ion signals studied for the dynamics of $\mathrm{C}_{2} \mathrm{H}_{4}\left(\mathrm{C}_{2} \mathrm{D}_{4}\right)$ are the parent ions (masses 28 and 32 respectively) and the fragments $\mathrm{C}_{2} \mathrm{H}_{3}{ }^{+}$, mass $27,\left(\mathrm{C}_{2} \mathrm{D}_{3}{ }^{+}\right.$, mass 30) and $\mathrm{C}_{2} \mathrm{H}_{2}{ }^{+}$, mass 26 , $\left(\mathrm{C}_{2} \mathrm{D}_{2}{ }^{+}\right.$, mass 28$)$, as functions of the probe delay, which was varied in steps of $3.3 \mathrm{fs}$. The time zero was determined from the maximum of the $\mathrm{Xe}^{+}$signal. The transient signals were simulated by the temporal function described in Chapter 3 (rate-equation model), that is, by a sum of exponentials convoluted with the instrumental function, the cross-correlation function between the VUV-excitation pulse $(162 \mathrm{~nm})$ and the second power of the probe-ionizing pulse at $810 \mathrm{~nm}$ (in the Franck-Condon region; a higher power of the probe for later $\mathrm{L}_{i}$ ). 
Fig. 4.3.1a, b shows the parent ion yields $\mathrm{C}_{2} \mathrm{H}_{4}{ }^{+}$and $\mathrm{C}_{2} \mathrm{D}_{4}{ }^{+}$. Obviously each exhibits a doubly exponential decay. Because the pump pulse gives rise to two different kinds of excited molecules, some in the $\pi \pi^{*}$ state and about $10 \%$ in the Rydberg state $\mathrm{L}_{R}$ (Fig. 4.2.2), the ion signals are expected to be the sum of two contributions, namely one from the direct excitation of the Rydberg state and subsequent relaxation from there and one from the direct excitation of the $\pi \pi^{*}$ state with subsequent relaxation; it seems obvious that the weaker signal (which in fact has an amplitude of $\approx 10 \%$ of the other) with the longer time constant ( $\tau_{R}=80$ and $115 \mathrm{fs}$ for $\mathrm{C}_{2} \mathrm{H}_{4}{ }^{+}$and $\mathrm{C}_{2} \mathrm{D}_{4}{ }^{+}$respectively) belongs to the Rydberg molecules. To isolate the shorterlived part, the two contributions need to be disentangled from each other. For this purpose, the signal at later times $(150-500 \mathrm{fs})$ is fitted by a single exponential function ( 80 fs and $115 \mathrm{fs}$ for $\mathrm{C}_{2} \mathrm{H}_{4}^{+}$and $\mathrm{C}_{2} \mathrm{D}_{4}^{+}$respectively), then convoluted with the instrumental function and extrapolated to negative times and finally subtracted from the total signal. The result is a single exponential decay $\left(\tau_{1}\right)$ with some modulation. It obviously reflects the relaxation from the $\pi \pi^{*}$ state (location $\mathrm{L}_{1}$ ). However, according to Fig. 4.2.3, the initial Rydberg $\left(\mathrm{L}_{R}\right)$ population flows to the $\pi \pi^{*}$ state and relaxes from there in the same manner as the molecules directly excited in the $\pi \pi^{*}$ state (with rate $\tau_{1}^{-1}$ ). To account for this part, the sum of the two exponential functions with equal amplitudes and with time constants $\tau_{R}$ and $\tau_{1}$, each convoluted with the instrumental function, is again fitted to the tail of the original signal and extrapolated till 20 fs: this curve reflects the contribution to the ion signal from a process in the neutral that involves excitation of the Rydberg state and consecutive population flow into, and out of, the $\pi \pi^{*}$ state $\left(\mathrm{L}_{1}\right)$ ("Rydberg path"). For this $\mathrm{L}_{R} \rightarrow \mathrm{L}_{1}$ relaxation and the ionization from $\mathrm{L}_{R}$ and $\mathrm{L}_{1}$ we assume that these two windows have the same probability for ionization, because they are almost isoenergetic. This simulation curve is again subtracted from the full signal (Fig. 4.3.1a, b). Such a flow, however, does not introduce new time constants: $L_{1}$ is in this model populated with time constant $\tau_{R}$ and depleted again within $\tau_{1}$ and so $\tau_{1}$ remains unchanged compared to the case, in which only a single $\tau_{R}$-exponential is subtracted. That is, the data would provide no information concerning the state towards which the $\mathrm{L}_{R}$ population is depleted, i.e. to an undetected state or to $L_{1}\left(\pi \pi^{*}\right)$. The latter seems, however, evident from the potentials of Fig. 4.2.3. 


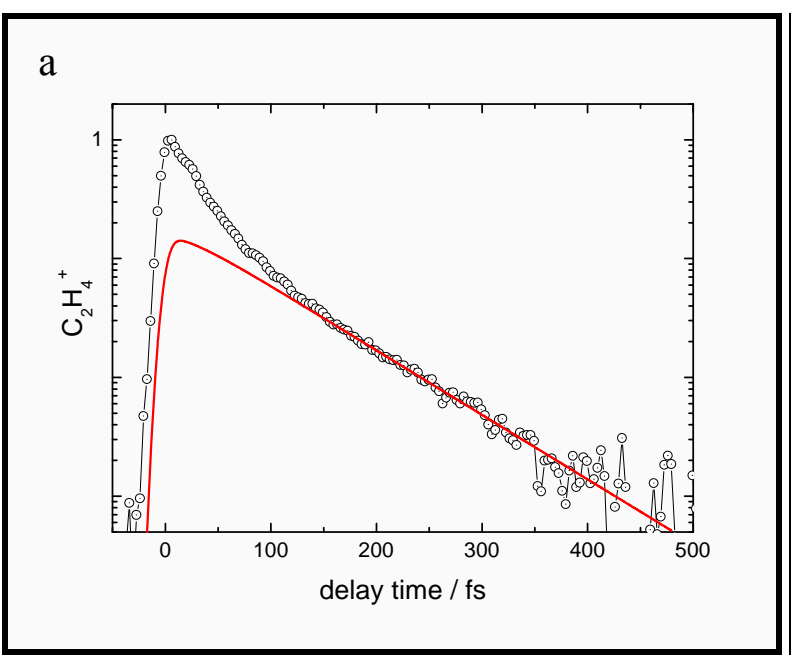

$\mathrm{b}$
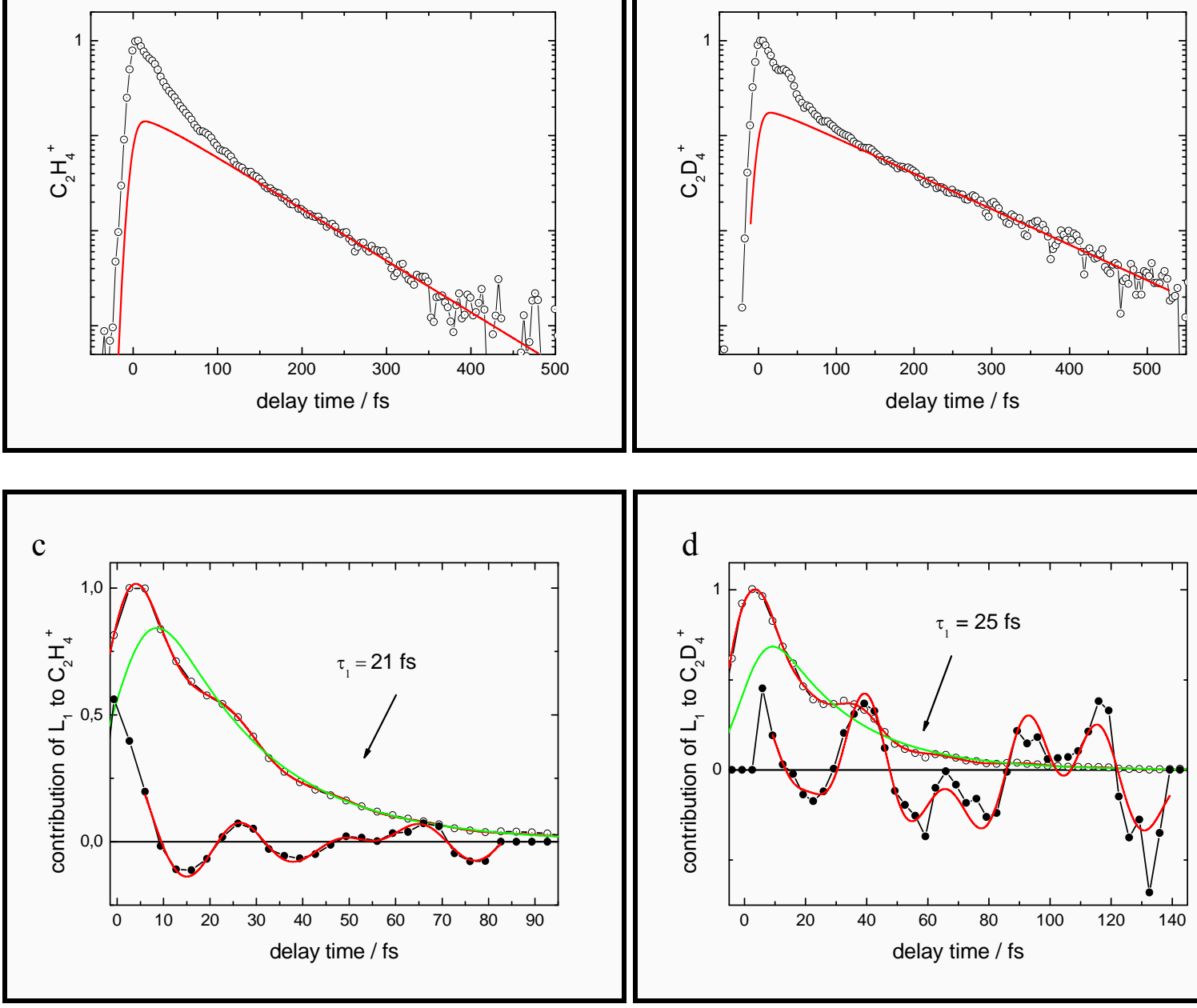

d

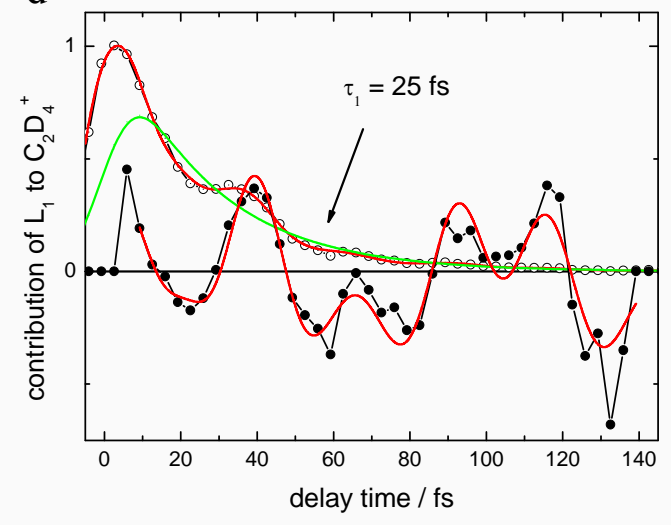

e

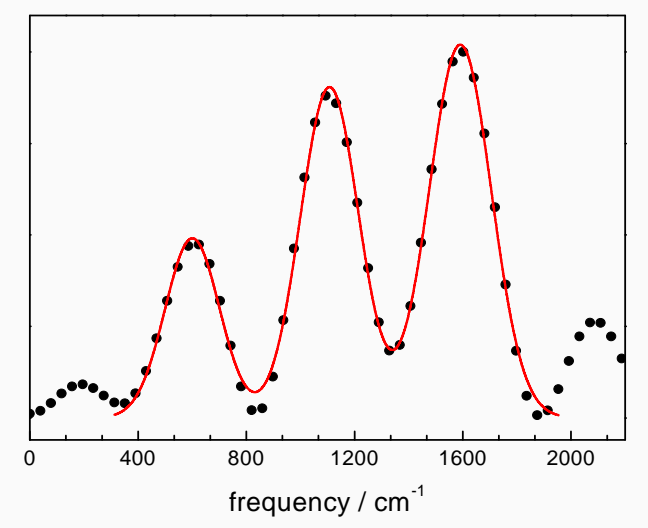

$\mathrm{f}$

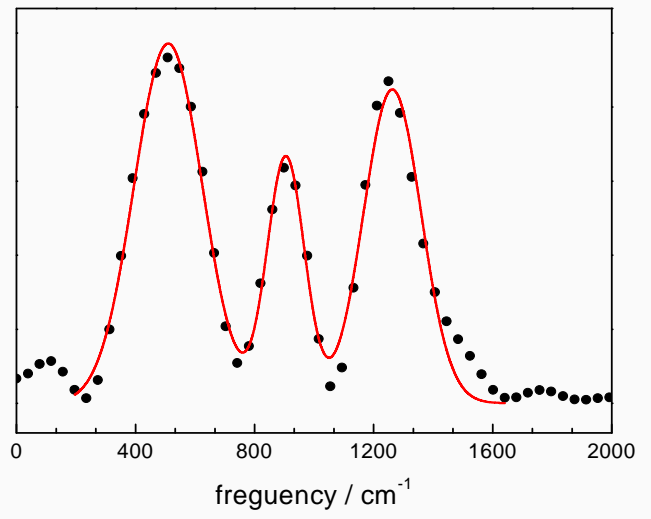

Fig. 4.3.1 Parent ion signals. a, c, e: $\mathrm{C}_{2} \mathrm{H}_{4}{ }^{+}, \mathrm{b}, \mathrm{d}, \mathrm{f}: \mathrm{C}_{2} \mathrm{D}_{4}{ }^{+}$; a, b: full signals and a simulation of the Rydbergpath contribution extrapolated to negative times; $\mathrm{c}$, d: signal (open symbols and signal simulation by the full-fit curve) after subtraction of the Rydberg-path contribution and a single exponential fit with convolution (green line); dividing these data by the latter fit curve and subtracting 1 results in the full symbols, which is fitted by an 
oscillatory function (see text below) with oscillation frequencies $v_{\mathrm{a}}, v_{\mathrm{b}}, v_{\mathrm{c}} ; \mathrm{e}$,f: Fourier transforms of the oscillatory functions in $\mathrm{c}$ and $\mathrm{d}$, fitted with the sum of three Gaussians.

The signal remaining after subtraction (Fig. $4.3 .1 \mathrm{c}, \mathrm{d}$ ) then reflects the population relaxation from $L_{1}$ after excitation of the $\pi \pi^{*}$ electronic state. It is in a first step simulated by one exponential decay (time constant $\tau_{1}$ ) convoluted with the instrumental function. In the second step, the data are divided by this fit curve resulting (after subtraction of 1) in the periodic functions at the bottom of Fig. 4.3.1c, d, the Fourier transform of which gives the frequencies of the modulated signal (Fig. 4.3.1e, f). These values are entered into functions of the type $f_{\text {osc }}=1+A \cos (2 \pi \mathrm{c} v t-\varphi)$, which are then multiplied by the (convoluted) exponential decay, and this complete function is then fitted to the measured data to get a final correction of the time constants $\tau_{i}$, the relative cross sections ${ }^{m} \sigma_{i}$ and the wavenumbers $v$; in contrast to previous cases [8] there is no additional dephasing term (exponential decay) before the cosine in the oscillatory equation, as it turned out that all detected oscillations decay with the lifetime of the population on the corresponding location. The extracted frequencies, time constants and other fit parameters (also for the fragment signals) are compiled in Table 4.3.1 below (p. 76).

Fig. 4.3.2a, $b$ shows the early-time data for the fragments $\mathrm{C}_{2} \mathrm{H}_{2}{ }^{+}$and $\mathrm{C}_{2} \mathrm{D}_{2}{ }^{+}$. This stronger earlier part also contains the time constants $\left(\tau_{1}, \tau_{R}\right)$ and modulation found already from the parent signal. Obviously the mass spectra of $\mathrm{L}_{1}$ and $\mathrm{L}_{R}$ also contain these fragment ions. To reveal additional information, we first subtract an exponential decay (with convolution) reflecting the Rydberg level contribution to the signal (lifetime $\tau_{R}$ ). The resulting signal originates only from the windows $\mathrm{L}_{1}$ and $\mathrm{L}_{2}$ with time constants $\tau_{1}$ and $\tau_{2}$, the second one being the lifetime of $\mathrm{L}_{2}$ (Fig. 4.3.2c, $\mathrm{d}$ open circles). Then the $\mathrm{L}_{1}$ contribution, i.e. an exponential with time constant $\tau_{1}$ modulated with frequencies $v_{\mathrm{a}}, v_{\mathrm{b}}$ and $v_{\mathrm{c}}$, is subtracted (green curve with early maximum and without data points in Fig. 4.3.2c, d). The residue is a doubly exponential function (time constants $\tau_{1}, \tau_{2}$ ) with a simple modulation of frequency $v_{\mathrm{d}}$ (full circles and the unmodulated curves with late maxima in Fig. 4.3.2c, d; shown also in a linear scale in Fig. 4.3.2e, f), which describes filling up a location $\mathrm{L}_{2}$ from $\mathrm{L}_{1}$ (with time constant $\tau_{1}$ ), an oscillation in this region and the depletion of $\mathrm{L}_{2}\left(\right.$ life time $\left.\tau_{2}\right)$. Fig. 4.3.2c, $\mathrm{d}$ shows, how these two contributions (from $\mathrm{L}_{1}$ and $\mathrm{L}_{2}$ ) add up to the total signal (from which the Rydberg-level contribution has been subtracted). Fig. 4.3.2e, f displays the $\mathrm{L}_{2}$ contribution once more (open cir- 
cles), the result of dividing it by the exponential fit (lifetime $\tau_{2}$ ) and, in the inset, the Fourier transform of this oscillatory part. Obviously, there is only a single oscillation frequency. As before, it was inserted in the time-dependent fit functions to simulate the partial and total signals, as shown in Fig. 4.3.2.
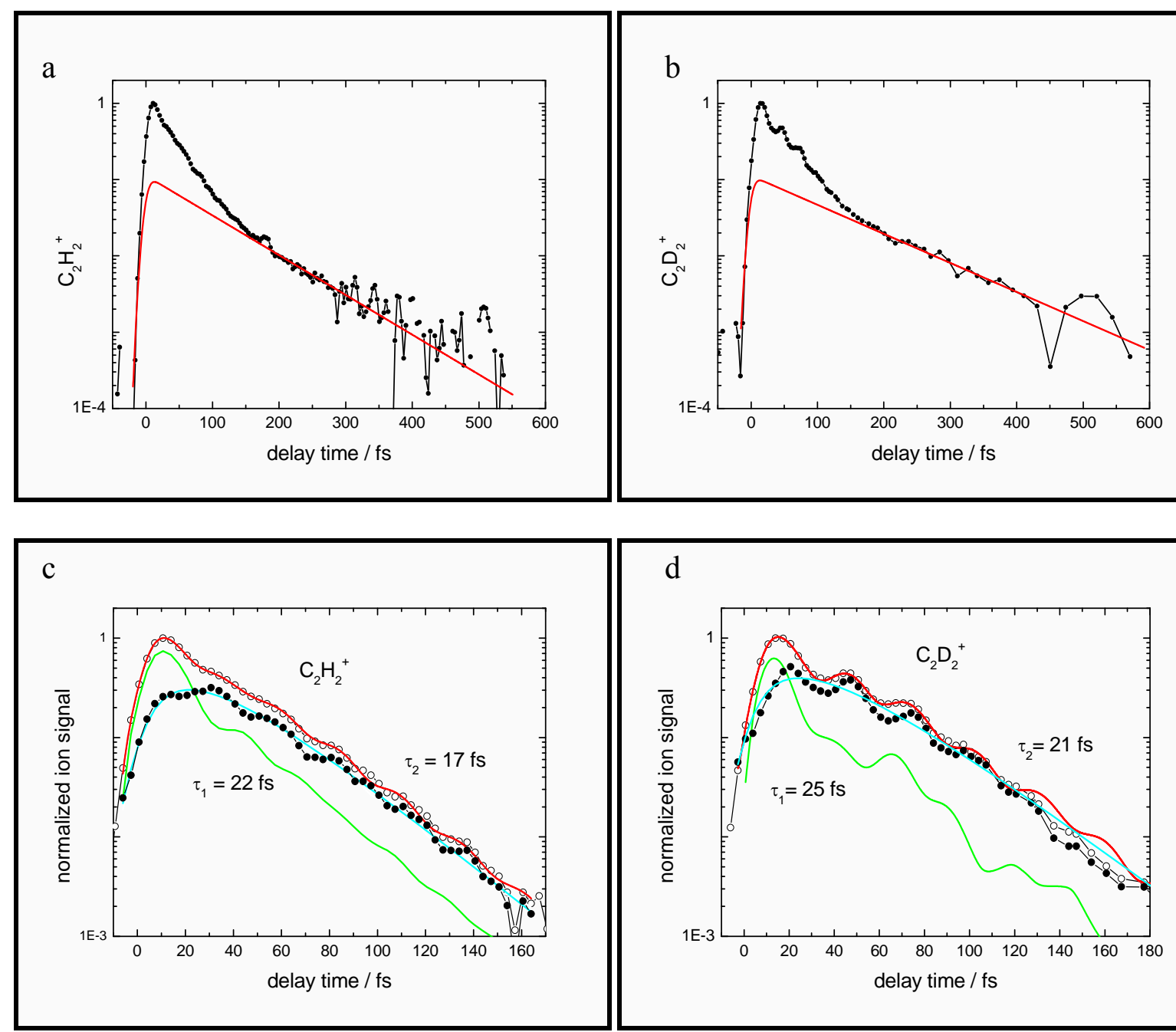

d
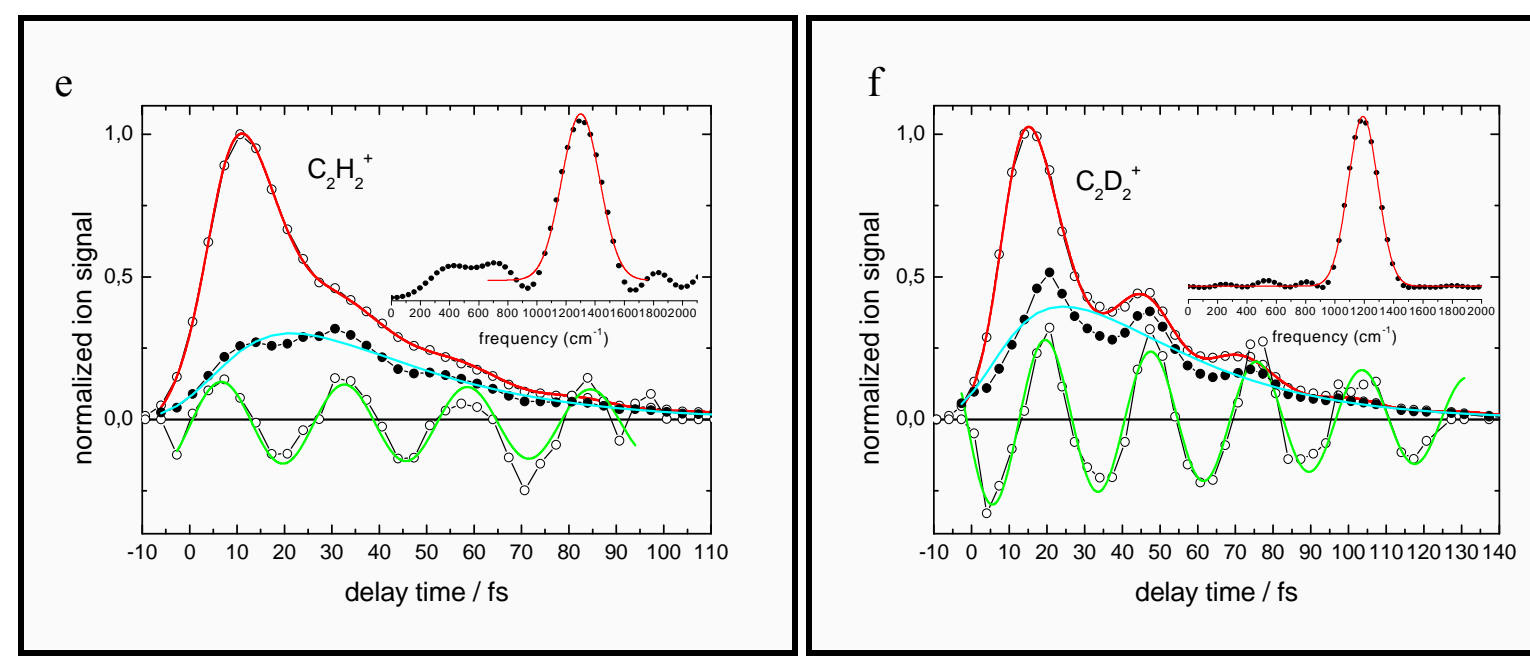
Fig. 4.3.2 Fragment ion signals. a, c, e: $\mathrm{C}_{2} \mathrm{H}_{2}{ }^{+}, \mathrm{b}, \mathrm{d}, \mathrm{f}: \mathrm{C}_{2} \mathrm{D}_{2}{ }^{+}$. a, b: full signals and a fit curve to the slower decay (time constant $\tau_{R}$, with convolution); c, d: signal (open circles) after subtraction of the slower decay, simulated by the full-fit curve; subtraction of the $\mathrm{L}_{1}$ contribution (curve with early maximum) yields the $\mathrm{L}_{2}$ contribution (full circles), which is fitted by exponentials (time constants $\tau_{1}, \tau_{2}$, with convolution); e, f: The original data after subtraction of the Rydberg contribution (open circles) and after subtraction of the $\mathrm{L}_{1}$ contribution (full circles) and their exponential simulation are again shown; dividing the $\mathrm{L}_{2}$ data by the fit curve (minus 1) results in the oscillatory part (open circles), whose Fourier transform is plotted in the inset; their simulation by an oscillatory function is also shown.

The $\mathrm{C}_{2} \mathrm{H}_{3}{ }^{+}\left(\mathrm{C}_{2} \mathrm{D}_{3}{ }^{+}\right)$fragment signal looks similar in this time range as the $\mathrm{C}_{2} \mathrm{H}_{2}{ }^{+}\left(\mathrm{C}_{2} \mathrm{D}_{2}{ }^{+}\right)$ signal (which has a better signal-to-noise ratio) and does not contain additional information. Its short-time part is therefore not shown.

To follow the "Rydberg path" contribution to the fragment ion signal (in the same way as in the parent ion case), one should now take into account three observation windows constituting the relaxation scheme $\mathrm{L}_{R} \rightarrow \mathrm{L}_{1} \rightarrow \mathrm{L}_{2}$. However, as explained previously, subtracting such a population flow from the original signal results in no additional information and the time constants and oscillation frequencies remain the same. Therefore, in the case of the fragment ions only one lifetime (i.e. of the Rydberg state, 80 and 115 fs for the two isotopomers) is convoluted, extrapolated to negative times and subtracted from the total signal.

At longer times, in the picosecond range, the two fragments reveal very weak tails, nonmodulated exponential decays with time constants $\tau_{3}$ and $\tau_{4}$. To record them, we raised the probe intensity by a factor of 3 (to $\approx 10^{14} \mathrm{~W} \mathrm{~cm}^{-2}$ ) and the pressure up to $\approx 10^{-4} \mathrm{mbar}$ (from the normally used $10^{-7}$ to $10^{-6}$ mbar) and did more averaging (Fig. 4.3.3). Whereas the $\mathrm{C}_{2} \mathrm{H}_{3}{ }^{+}$signal shows both $\tau_{3}$ and $\tau_{4}$, the fragment $\mathrm{C}_{2} \mathrm{H}_{2}{ }^{+}$only contains $\tau_{3}$. The effect of the probe intensity is demonstrated with $\mathrm{C}_{2} \mathrm{D}_{3}{ }^{+}$as an example, in Fig. 4.3.3b, where the small symbols show data recorded with standard probe intensity $\left(\approx 3 \times 10^{13} \mathrm{~W} \mathrm{~cm}^{-2}\right)$. Evidently, reducing the intensity by a factor of 3 lowers the ion yields to levels where $\tau_{3}$ can barely be recognized and the $\mathrm{L}_{4}$ window $\left(\tau_{4}\right)$ is below the detection limit.

The parent-ion signal decays to 0 . That is, the ion yields at negative and long positive delay times coincide. The former is obviously due to multi-photon ionization by the probe and is subtracted from the measured data. For the fragment ions the long-time signals are slightly higher (about $10^{-4}$ of the maximum) than those at negative time. Because this "pedestal" de- 
pends nonlinearly on the UV intensity (varied by a factor of 2, by changing the Ar pressure in the fifth-harmonic cell), it cannot be due to one-photon excited molecules; therefore we subtracted it from the fragment signals. All the rest, i.e. the signals from all $\mathrm{L}_{i}$, are linear in the pump intensity and the ratios of signals stay perfectly constant. The absence of a long-time signal means that no end product was detected.

The probe energy was also varied by a factor of 3. An example is shown in Fig. 4.3.3b. The corresponding variation of the signal intensity (more precisely: the ionization probability) could inform on the order of ionization of the different $\mathrm{L}_{i}$ and hence on their relative energies. For this purpose, one should add up the parent and fragment signals; unfortunately each signal was separately measured and normalized. We instead compare the dominant signals, which now contain the contributions from all the observation windows (i.e. there is no subtraction of the Rydberg state contribution) measured up to several picoseconds: the parent from $\mathrm{L}_{1}$ and $\mathrm{L}_{R}$ and $\mathrm{C}_{2} \mathrm{H}_{2}^{+}$from $\mathrm{L}_{2}$ and $\mathrm{L}_{3}$. The former pair was found to have the same order of ionization (the signal ratios do not change with the probe energy, Table 4.3.1), so that the two locations have roughly the same energy. As for $\mathrm{L}_{3}$ and $\mathrm{L}_{4}$, the probe-intensity dependence is less conclusive, probably because the fragmentation pattern also changes. Nevertheless it can be recognized from Table 4.3.1 that $\mathrm{L}_{3}$ and $\mathrm{L}_{4}$ are below $\mathrm{L}_{2}$, because the signals from the former are by more than two orders of magnitude weaker than those from $\mathrm{L}_{2}$.

One could expect that the ${ }^{m} \sigma_{i}$ from the corresponding locations $\mathrm{L}_{i}$ coincide for the two isotopomers. This is the case within a factor of 2 for the higher probe intensity (Table 4.3.1). For the fragments at lower intensity there are more deviations, perhaps due to their strong sensitivity to the intensity. (The intensity might have been slightly different from day to day.)
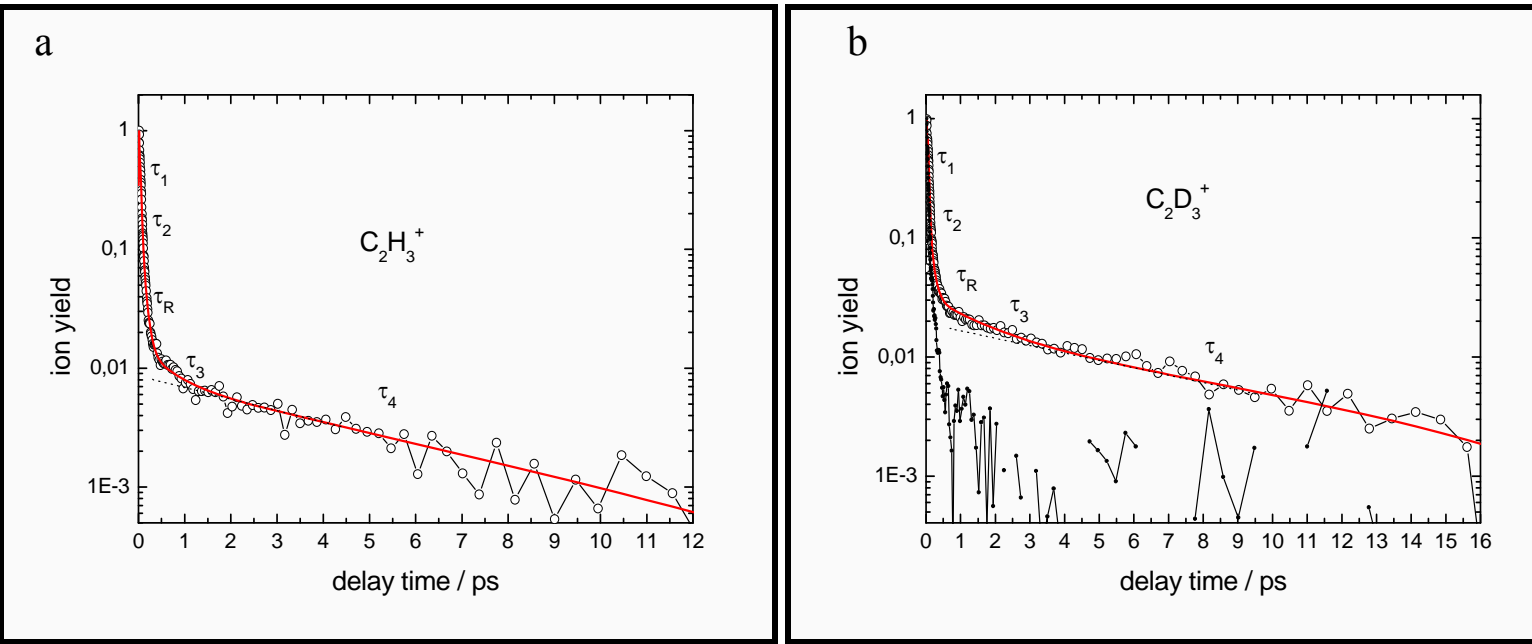

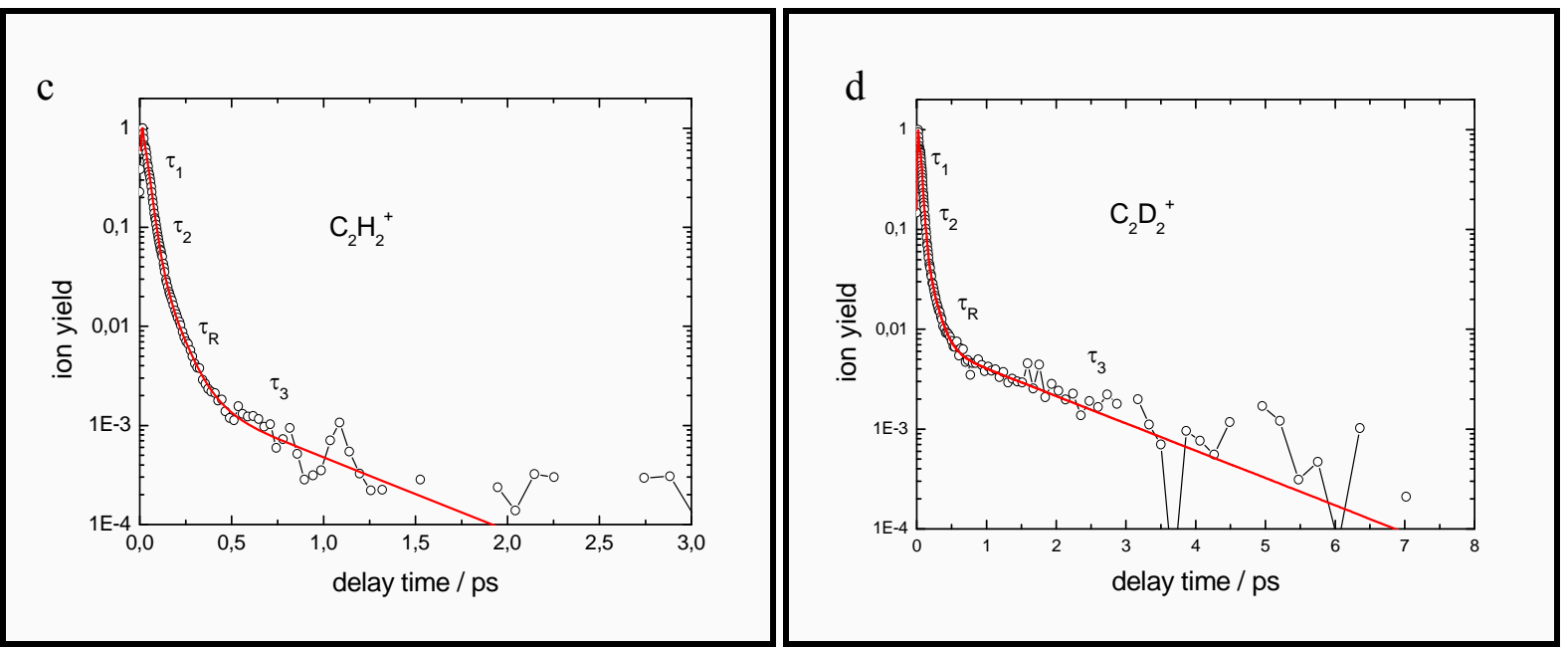

Fig. 4.3.3 Signals of fragment ions (as indicated) at longer times, showing the time constants $\tau_{3}$ and $\tau_{4}$; the shorter time constants $\tau_{1}, \tau_{2}$ and $\tau_{R}$ are derived from the signal parts in Fig. 4.3.1 and 2. Note that the time scales are different. $\tau_{4}$ shows up only in the $\mathrm{C}_{2} \mathrm{H}_{3}{ }^{+}\left(\mathrm{C}_{2} \mathrm{D}_{3}{ }^{+}\right)$signal. The data were recorded with $\approx 3$ times higher probe intensity $\left(\approx 10^{14} \mathrm{~W} \mathrm{~cm}^{-2}\right)$ than usual, which improves the signal-to-noise ratio significantly; as an example, data for standard probe intensity are also shown for $\mathrm{C}_{2} \mathrm{D}_{3}{ }^{+}$(small full circles).

\begin{tabular}{|c|c|c|c|c|c|}
\hline $\begin{array}{ll}\mathrm{L}_{i} & \left(\mathbf{C}_{2} \mathbf{H}_{4}\right) \\
\tau_{i} & \\
\end{array}$ & $\begin{array}{c}\mathrm{L}_{R} \\
80 \mathrm{fs}\end{array}$ & $\begin{array}{c}\mathrm{L}_{1} \\
21.4 \mathrm{fs}\end{array}$ & $\begin{array}{c}\mathrm{L}_{2} \\
16.5 \mathrm{fs}\end{array}$ & $\begin{array}{c}\mathrm{L}_{3} \\
0.60 \mathrm{ps}\end{array}$ & $\begin{array}{c}\mathrm{L}_{4} \\
5.0 \mathrm{ps}\end{array}$ \\
\hline \multirow[t]{2}{*}{$\mathrm{C}_{2} \mathrm{H}_{4}^{+}(28 \mathrm{u})$} & 0.1 & 1 & 0 & 0 & 0 \\
\hline & 0.07 & 1 & 0 & 0 & 0 \\
\hline \multirow[t]{2}{*}{$\mathrm{C}_{2} \mathrm{H}_{3}^{+}(27 \mathrm{u})$} & 0.08 & 1 & 0.3 & 0.004 & 0.003 \\
\hline & 0.2 & 1 & 2.3 & 0.018 & 0.008 \\
\hline \multirow[t]{2}{*}{$\mathrm{C}_{2} \mathrm{H}_{2}^{+}(26 \mathrm{u})$} & 0.07 & 1 & 0.7 & 0.001 & 0 \\
\hline & 0.08 & 1 & 1.6 & 0.002 & 0 \\
\hline$v / \mathrm{cm}^{-1}$ & & $\begin{array}{c}631,1113,1572 \\
\left(v_{\mathrm{a}}, v_{\mathrm{b}}, v_{\mathrm{c}}\right)\end{array}$ & $\begin{array}{l}1263 \\
\left(v_{\mathrm{d}}\right)\end{array}$ & & \\
\hline \multirow[t]{2}{*}{$\mathrm{L}_{i} \quad\left(\mathbf{C}_{2} \mathbf{D}_{4}\right)$} & $\mathrm{L}_{R}$ & $\mathrm{~L}_{1}$ & $\mathrm{~L}_{2}$ & $\mathrm{~L}_{3}$ & $\mathrm{~L}_{4}$ \\
\hline & $115 \mathrm{fs}$ & $24.2 \mathrm{fs}$ & $21 \mathrm{fs}$ & $1.6 \mathrm{ps}$ & $11 \mathrm{ps}$ \\
\hline $\mathrm{C}_{2} \mathrm{D}_{4}^{+}(32 \mathrm{u})$ & 0.1 & 1 & 0 & 0 & 0 \\
\hline \multirow[t]{2}{*}{$\mathrm{C}_{2} \mathrm{D}_{3}^{+}(30 \mathrm{u})$} & 0.2 & 1 & 4.2 & 0.01 & $5 \times 10^{-5}$ \\
\hline & 0.1 & 1 & 1.2 & 0.026 & 0.006 \\
\hline \multirow[t]{2}{*}{$\mathrm{C}_{2} \mathrm{D}_{2}^{+}(28 \mathrm{u})$} & 0.1 & 1 & 2.7 & 0.002 & 0 \\
\hline & 0.1 & 1 & 2.5 & 0.007 & 0 \\
\hline$v / \mathrm{cm}^{-1}$ & & $\begin{array}{c}512,891,1263 \\
\left(v_{\mathrm{a}}, v_{\mathrm{b}}, v_{\mathrm{c}}\right)\end{array}$ & $\begin{array}{l}1190 \\
\left(v_{\mathrm{d}}\right)\end{array}$ & & \\
\hline
\end{tabular}


Table 4.3.1 Lifetimes $\tau_{i}$ of population in the locations $\mathrm{L}_{i}$, ionization cross-sections ${ }^{m} \sigma_{i}$ to produce an ion of mass $m$ ( $m$ is indicated in parenthesis in column 1) from $L_{i}$ and the oscillation frequencies from $L_{i}$. Estimated error limits for the time constants and frequencies are $\pm 5 \%$. The ${ }^{m} \sigma_{i}$ printed in italic are for a probe intensity of $\approx 10^{14} \mathrm{~W} \mathrm{~cm}^{-2}$, the others for $\approx 3 \times 10^{13} \mathrm{~W} \mathrm{~cm}^{-2}$. The picosecond time constants $\tau_{3}$ and $\tau_{4}$ were determined with the higher intensity, which raises the corresponding signals.

In context with the isotope shifts, the error limits of the time constants and frequencies would be interesting. In previous experiments we used for this purpose the standard deviation found, when each of $\geq 10$ scans was evaluated and the results averaged. In the present case this is difficult, however, due to the multistep evaluation procedure (subtraction of the $\mathrm{L}_{R}$ contribution, for $\mathrm{L}_{2}$ also subtraction of the $\mathrm{L}_{1}$ contribution, division by the exponential parts, etc.); as a consequence the analysis of each scan is not only tedious, but the errors of the different quantities will also be interdependent. To get nevertheless an estimate at least for the more important numbers $\left(\tau_{1}, \tau_{2}\right.$ and the vibrational wavenumbers), we instead average the measured data of 8 scans (= one set of data) and then evaluate them. Then another such set is evaluated (for $\mathrm{C}_{2} \mathrm{H}_{4}$ it was measured at the higher probe intensity, for $\mathrm{C}_{2} \mathrm{D}_{4}$ on a different day) and the results are then compared. The $( \pm)$ deviation from the average was

- for $\mathrm{C}_{2} \mathrm{H}_{4}: 3 \%$ for $\tau_{1}$ and $\tau_{2} ; 5 \%, 0.5 \%, 1 \%$ and $3 \%$ for $v_{\mathrm{a}}-v_{\mathrm{d}}$,

- for $\mathrm{C}_{2} \mathrm{D}_{4}: 4 \%$ for $\tau_{1} ; 0.6 \%$ and $3 \%$ for $v_{\mathrm{a}}$ and $v_{\mathrm{b}}$.

In view of the small deviations it is probably safe to estimate an error limit of $\leq 5 \%$, also for the time constants and wavenumbers, for which the fluctuation was not investigated.

\subsection{Discussion}

\subsubsection{Assignments of the lifetimes and oscillations}

The observation windows $\mathrm{L}_{i}$ are locations on the potential surfaces, which are to be identified by the assignment. Their population lifetimes $\tau_{i}$, the oscillation frequencies and the different mass spectra (fragmentation patterns), ionization probabilities and orders of ionization associated with different $\mathrm{L}_{i}$, help to assign them to different regions on the potential energy surfaces (PES): electronic relaxation lowers the electronic energy and therefore reduces the ionization probability (signal intensity) and raises the order of ionization; the latter is determined from observing the signal on variation of the probe intensity. Due to energy conservation during relaxation, vibrational excess energy is released and ionization of such a hot molecule gives 
rise to a hot ion that fragments before detection; the degree of fragmentation of the parent ion is thus also a measure of the electronic (or vibrational excess) energy of an individual $\mathrm{L}_{i}$. The fit parameters ${ }^{m} \sigma_{i}$ reflect both the ionization probability and the fragmentation pattern (for details see [34]).

The coherent oscillations result from superposition of stationary vibrational states of the molecule in the location $\mathrm{L}_{i}$. They can contribute to the assignment of $\mathrm{L}_{i}$ on the PES. However, there is a chance to observe an oscillation only, if the vibration modulates the ionization or fragmentation probability. This is not expected, for example, if in the probe transition the lower and upper potentials are parallel, as is the case on ionization from a Rydberg state. There are also a number of other reasons why some oscillations may remain unobserved (see Chapter 3, sec. 3.4.3).

For the assignment we start with the knowledge of the potentials from spectroscopy and quantum chemistry (Fig. 4.2.3). Initial excitation at $162 \mathrm{~nm}$ mainly populates the one-electron excited $\pi \pi^{*}(\mathrm{~V})$ state and the $\pi 3 s$ Rydberg state with 5-10 times less probability. Population can flow easily (i.e., with only very minor activation energy) from the Rydberg state to the $\pi \pi^{*}$ surface and from there further down (Fig. 4.2.3). According to the present understanding [68], the way down from the Franck-Condon region of the $\pi \pi^{*}$ state does not only involve CC torsion $\varphi$ (which gives rise to a horizontal tangent at $\varphi=0$ and hence to only slow initial acceleration) but also CC stretching (not indicated in Fig. 4.2.3). Near $\varphi=90^{\circ}$, the $\mathrm{V}$ and $\mathrm{Z}$ states become nearly degenerate and easily exchange population. After reaching $\varphi=90^{\circ}$ the molecule starts taking a new relaxation direction to reach a conical intersection $(\mathrm{CI})$ with the ground-state $\left(\mathrm{S}_{0}\right)$ surface. The new direction involves pyramidalization of one $\mathrm{CH}_{2}$ group and/or partial migration of a $\mathrm{H}$ atom from one $\mathrm{C}$ to the other. After passing through the $\mathrm{CI}$, the molecule can either directly reach the $\mathrm{S}_{0}$ minimum of $\mathrm{C}_{2} \mathrm{H}_{4}$ or - if $\mathrm{H}$ migration is involved temporarily arrive at a structure corresponding to the carbene $\mathrm{CH}_{3} \mathrm{CH}$ (ethylidene, not indicated in Fig. 4.2.3). Ethylidene very rapidly rearranges on a barrierless path in the ground state back to ethylene. In the hot $\mathrm{S}_{0}, \mathrm{H}$ and $\mathrm{H}_{2}$ eliminations can take place.

\subsubsection{The Franck-Condon region}

The parent-ion signal contains strong contributions from observation windows $\left(\mathrm{L}_{R}\right.$ and $\left.\mathrm{L}_{1}\right)$ with time constants $\tau_{R}$ and $\tau_{1}$, whereas it does not show the time constants $\tau_{2}-\tau_{4}$. That is, from 
these locations the ionization probability is highest and the fragmentation probability lowest. Obviously they represent the Franck-Condon region. The signal contribution from $\mathrm{L}_{R}$ is about 10 times smaller than that from $\mathrm{L}_{1}$, depending to some extent on the probe intensity. This is practically the same as the estimated ratio $(5-10)$ of the excitation probabilities of the $\pi \pi^{*}$ and $\pi 3 s$ states (see above and Fig. 4.2.2). Therefore we assign $\mathrm{L}_{R}$ to the Rydberg $\pi 3 s$ state (lifetimes $\tau_{R}=80$ fs and 115 fs for $\mathrm{C}_{2} \mathrm{H}_{4}$ and $\mathrm{C}_{2} \mathrm{D}_{4}$, respectively) and $\mathrm{L}_{1}$ to the $\pi \pi^{*}$ state (lifetimes $\tau_{1}=21 \mathrm{fs}$ and $24 \mathrm{fs}$, respectively).

The oscillations, which are observed in the $L_{1}$ window but not in $L_{R}$, support this assignment: although vibrational coherence is certainly also generated on exciting the Rydberg state, it should not be observed on probing by ionization, because of the parallel potentials, as explained above. On excitation of the $\pi \pi^{*}$ state, a coherent superposition can be expected for the Franck-Condon active vibrations. This will mainly be torsion (twist, $v_{4}$ ) and probably CC stretch $\left(v_{2}\right)$ too; the resonance-Raman spectrum suggests additionally some activity of the symmetric scissors vibration $\left(v_{3}\right)$ and its antisymmetric counterpart $\left(v_{12}\right)$ and the out-of-plane wagging $\left(v_{7}\right)[88,89]$. In $\mathrm{S}_{0}$, full deuterium substitution reduces all these frequencies by a factor of 1.41-1.33 except for the CC stretch vibration $\left(v_{2}\right)$, which is reduced by a factor of 1.07 [104], as the inverse ratio of the square root of the moving masses. For the coherent oscillations $v_{\mathrm{a}}, v_{\mathrm{b}}$ and $v_{\mathrm{c}}$ observed in the $\pi \pi^{*}$ state $\left(\mathrm{L}_{1}\right)$ the corresponding factors are 1.18, 1.22 and 1.26, respectively (Table 4.3.1). Hence none of them involves a pure hydrogen vibration; all must be mixed and contain some $\mathrm{CC}$ stretch. From the wavenumbers it might be supposed that the $\mathrm{CC}$ stretch dominates in $v_{\mathrm{b}}\left(1110 \mathrm{~cm}^{-1}\right.$ in $\left.\mathrm{C}_{2} \mathrm{H}_{4}\right)$; then the torsion will dominate in $v_{\mathrm{a}}\left(630 \mathrm{~cm}^{-1}\right.$ in $\left.\mathrm{C}_{2} \mathrm{H}_{4}\right)$, and $v_{\mathrm{c}}\left(1570 \mathrm{~cm}^{-1}\right.$ in $\left.\mathrm{C}_{2} \mathrm{H}_{4}\right)$ is the combination vibration of the two. The latter wavenumber would be too high to assign it to a CC stretch (ground-state value $1623 \mathrm{~cm}^{-1}$ [104], which should be drastically reduced in a $\pi$-antibonding state). The wavenumber of $v_{\mathrm{c}}$ is by only $9 \%$ smaller than the sum $v_{\mathrm{a}}+v_{\mathrm{b}}$, which is in the range of common anharmonic shifts.

This assignment is further supported by the recurrence times of the wave packets calculated after excitation to this energy region by Viel et al. and Brill et al. [100, 105]: the CC distance oscillates with a period of 25-30 fs, corresponding to a wavenumber of $1330-1110 \mathrm{~cm}^{-1}$; the torsion has a recurrence time near $45 \mathrm{fs}\left(740 \mathrm{~cm}^{-1}\right)$. The calculation also predicts a small 
amplitude for the scissors vibrations (similar frequency as $\mathrm{CC}$ stretch; but note that we need a strong participation of the $\mathrm{CC}$ stretch vibration to explain the small isotope shift that we observed) and a practically negligible amplitude for wagging. Moreover, the calculation points out that the $\mathrm{CC}$ distance shortens on twisting (after lengthening at the initially zero twist), so that the two motions are not separable. This can explain the mixed character of the vibrations, concluded above from the small isotope shift, and also the observation of a combination vibration. Finally, Barbatti et al. find in their surface-hopping study a periodicity of $\approx 20 \mathrm{fs}$ $\left(1660 \mathrm{~cm}^{-1}\right.$, well consistent with our combination vibration of $\left.1570 \mathrm{~cm}^{-1}\right)$ in the $\mathrm{S}_{1}$ population, after excitation at $160 \mathrm{~nm}$ (Fig. 3 in [106]); it must reflect a vibration (in the Franck-Condon region), since $\mathrm{V} \leftrightarrow \mathrm{Z}$ electronic relaxation only takes place near $\varphi=90^{\circ}$. Our findings thus also confirm the quantum chemical calculations of $[100,105,106]$.

In the spectra (Fourier transforms, Fig. 4.3.1e, f) of the oscillations in the Franck-Condon region the relative intensity of the combination frequency over that of the fundamentals is not the same for $\mathrm{C}_{2} \mathrm{D}_{4}$ and $\mathrm{C}_{2} \mathrm{H}_{4}$. This is not unexpected, if the anharmonic interaction between $\mathrm{CC}$ stretch and torsion differs for the two isotopomers. Such interactions usually depend on frequency differences and hence on isotopic substitution. Probably one can interpret in the same way the different intensity ratios of $\mathrm{CC}$ stretch (which is weak in $\mathrm{C}_{2} \mathrm{D}_{4}$ ) over that of CC torsion (which is weak in $\mathrm{C}_{2} \mathrm{H}_{4}$ ). The narrow width of the $\mathrm{CC}$ stretch of $\mathrm{C}_{2} \mathrm{D}_{4}$ (Fig. 4.3.1f) is probably an artifact of the Fourier transform, because it is not reflected in the dephasing time in the simulation of the time-dependent data. Also the peak near $2100 \mathrm{~cm}^{-1}$ for $\mathrm{C}_{2} \mathrm{H}_{4}$ may be spurious, although it would fit to the next member $\left(\mathrm{v}_{4}=2\right)$ in the sequence $v_{2}+\mathrm{v}_{4} v_{4}$.

\subsubsection{Departure from $\mathrm{S}_{1}$}

The time constant $\tau_{2}$ is not contained in the parent-ion signal but only in the two fragments. This means that from $\mathrm{L}_{2}$ ionic fragmentation is complete (in contrast to $\mathrm{L}_{1}$ and $\mathrm{L}_{\mathrm{R}}$, from which the parent ion dominates, Table 4.3.1), indicating that $\mathrm{L}_{2}$ is much lower in energy than the Franck-Condon region. Because the next two windows $\left(\mathrm{L}_{3}\right.$ and $\left.\mathrm{L}_{4}\right)$ are already on the $\mathrm{S}_{0}$ surface (see below), $\tau_{2}$ must be the time for leaving $\mathrm{S}_{1}$. The oscillation observed in $\mathrm{L}_{2}\left(v_{\mathrm{d}}=1260\right.$ $\mathrm{cm}^{-1}$ and $1190 \mathrm{~cm}^{-1}$ for $\mathrm{C}_{2} \mathrm{H}_{4}$ and $\mathrm{C}_{2} \mathrm{D}_{4}$, respectively) must then be a vibration in the lower part of the $\mathrm{S}_{1}$ surface. The isotope shift $(6 \%)$ indicates that it is a pure $\mathrm{CC}$ stretch vibration. Interestingly, Foo and Innes found in the long-wavelength part of the UV spectrum of $C_{2} D_{4} a$ progression displaced by $1250 \mathrm{~cm}^{-1}$ from the main $\left(v_{4}\right)$ progression [87]. They assign this 
shift to a $v_{2}$ quantum, i.e. to a CC stretch vibration in the lower part of $\mathrm{S}_{1}$. The agreement with our $1190 \mathrm{~cm}^{-1}$ seems good enough to support our identification of $v_{\mathrm{d}}$ and location $\mathrm{L}_{2}$. Furthermore, the increase of the $\mathrm{CC}$ stretch wavenumber from $\mathrm{L}_{1}$ to $\mathrm{L}_{2}\left(v_{\mathrm{b}}\right.$ compared to $\left.v_{\mathrm{d}}\right)$ by 20 $30 \%$ is fully consistent with the theoretical prediction $[92,93,100,107]$ that the CC bond is shorter at $\varphi=90^{\circ}$ than at $0^{\circ}$, implying a stronger bond in the twisted structure. It is even more strengthened and shortened, when a $\mathrm{H}$ bridge forms on the way to $\mathrm{H}$ migration [92].

The agreement of the $\mathrm{CC}$ stretch frequency found here with the spectroscopic value for the region near the perpendicular $\mathrm{S}_{1}$ "minimum" $\left(\mathrm{C}_{2} \mathrm{H}_{4}\right.$ with $D_{2 \mathrm{~d}}$ symmetry) does not necessarily mean that $\mathrm{L}_{2}$ is exactly there though. If $\tau_{2}$ is the time for leaving $\mathrm{S}_{1}, \mathrm{~L}_{2}$ should extend to the $\mathrm{S}_{1} /$ $\mathrm{S}_{0}$ conical intersection (CI); a change of ionization probability (hence a termination of the observation window $\mathrm{L}_{2}$ ) will take place especially there, where the electronic energy quickly drops and there is change from one electronic potential to another. Hence apart from the $90^{\circ}$ torsion in $\mathrm{L}_{2}$ there will also be some pyramidalization and/or $\mathrm{H}$ migration. In fact, quantum chemistry predicts that the $D_{2 \mathrm{~d}}$ structure is not a minimum (which would imply some residence time) but a saddle point [93, 97, 99, 107].

During a drastic geometrical change such as pyramidalization and/or H migration any initial twist vibration will strongly change its character and wavenumber (from an initial value near $800 \mathrm{~cm}^{-1}$ in the $D_{2 \mathrm{~d}}$ geometry $\left.[87,108]\right)$. Furthermore, near this structure the zwitterionic state begins to be populated; the twist vibration has a much higher frequency there (note the strong curvature of the $\mathrm{Z}$ potential in Fig. 4.2.3), with the result of a rapid loss of any initial coherence due to these frequency variations. It is therefore not surprising that a coherent twist oscillation $v_{4}$ and its combination with CC stretching $\left(v_{2}\right)$ is not observed from $\mathrm{L}_{2}$. (In fact, a $v_{4}$ oscillation has been observed in $\mathrm{S}_{1}$ of $\mathrm{C}_{2} \mathrm{~F}_{4}$, where the zwitterionic state seems to be higher in energy and not involved in the relaxation process [109].) It is also not surprising that any $v_{2} / v_{4}$ mixing is completely different between the two windows $\mathrm{L}_{1}$ and $\mathrm{L}_{2}$, as indicated by the different isotope effect. In $\mathrm{L}_{1}$ the twist is a hindered free rotation; along the large angular excursion the $\mathrm{CC}$ distance changes (see above) so that the two motions couple. In $\mathrm{L}_{2}$ the twist amplitude is much smaller, so that the $\mathrm{CC}$ distance remains constant and the two motions are decoupled. 
In a previous investigation of the ultrafast dynamics of ethylene (pumping by 130-fs pulses at $198 \mathrm{~nm}$ ), Stert et al. [84] found a second time constant with values ( $\tau_{2} \approx 15$ and 25 fs for $\mathrm{C}_{2} \mathrm{H}_{4}$ and $\mathrm{C}_{2} \mathrm{D}_{4}$, respectively) very similar to ours. The cutoff in the photoelectron spectrum, measured by a coincidence technique from this window, indicates that the probe laser (792 $\mathrm{nm}, 1.56 \mathrm{eV}$ ) does not reach the energy minimum of the parent ion but a higher state. Indeed, the electronic ground state of the ion is raised by geometric distortion. In Fig. 4.4.1.2.1a, we adopt the calculated ion energies of $1.3 \mathrm{eV}$ (above the ion ground state) for the $D_{2 \mathrm{~d}}$ (perpendicularly twisted) geometry [110] and the value 1.2-1.4 eV calculated for $\mathrm{CH}_{3} \mathrm{CH}^{+}$or a bridged variant of it [111]; these energies are $11.8 \mathrm{eV}$ above neutral $\mathrm{C}_{2} \mathrm{H}_{4}$. Using the $0.85 \mathrm{eV}$ cut-off energy of the photoelectrons in five-photon ionization [84], we obtain an electronic energy of $\mathrm{L}_{2}$ of $4.8 \mathrm{eV}$ (Fig. 4.4.1.2.1b). (We tacitly assume ionization with $\Delta v=0$, i.e. parallel potentials for the neutral molecule and ion in this region. This assumption seems necessary to explain the sharp cutoff observed.) This value is entirely conceivable for the region between the perpendicular $D_{2 \mathrm{~d}}$ minimum $(5.5 \mathrm{eV})$ [87] and the $\mathrm{S}_{1} / \mathrm{S}_{0} \mathrm{CI}$; furthermore it is fully consistent with the twisted-pyramidalized CI of $4.5 \mathrm{eV}$ of the recent high-level calculations [92] (see also Figs. 5 and 6 there [92]). This experimental evidence hence also supports the assignment of $\mathrm{L}_{2}$ to a region extending to the last CI from around the $D_{2 \mathrm{~d}}$ minimum.

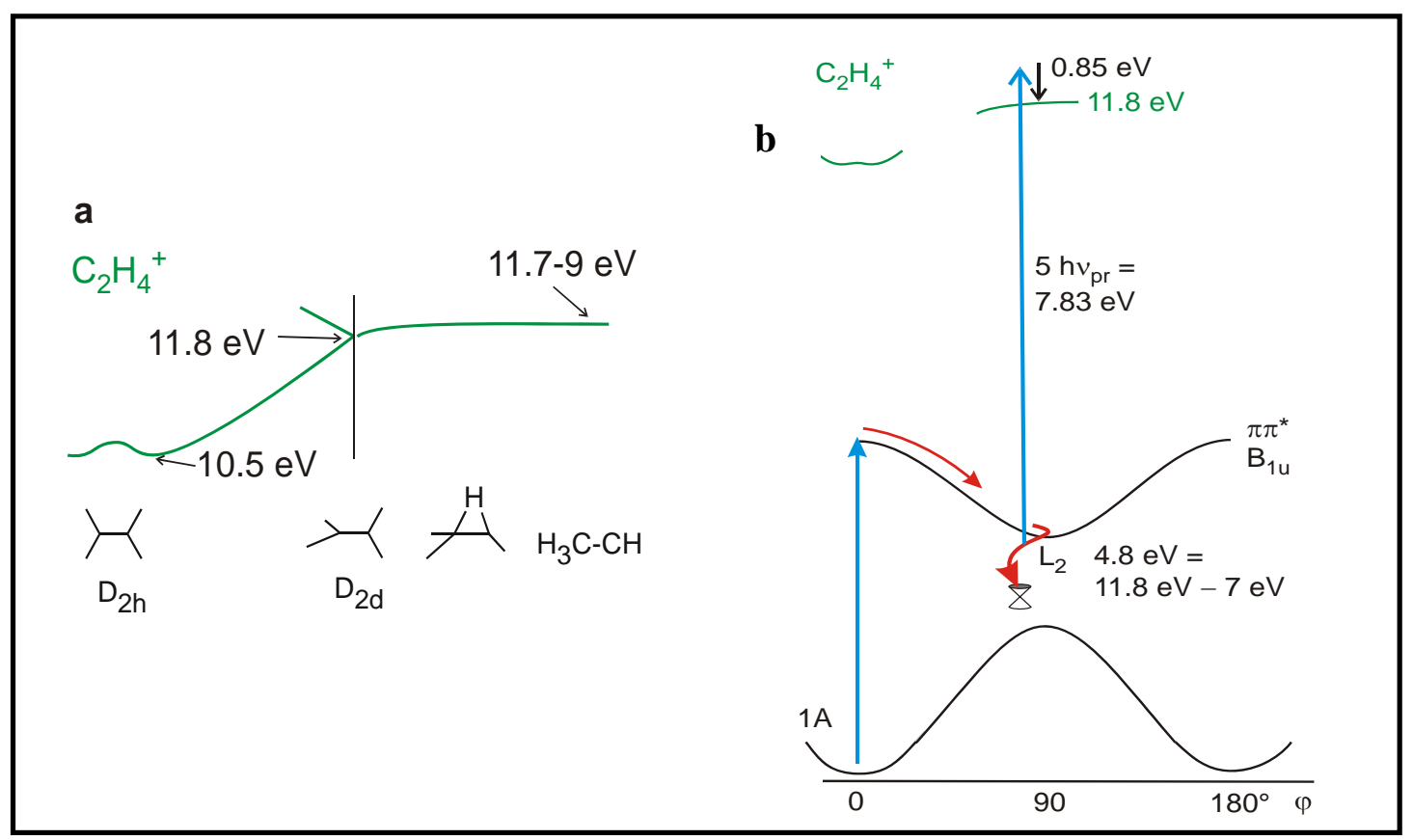

Fig. 4.4.1.2.1 (a) Ion energies relative to the neutral ground state. In $D_{2 \mathrm{~d}}$ geometry (calculation by Sannen et al. [110]), the electronic state is degenerate. From there, $\mathrm{H}$ migration does not much affect the energy according to the calculation of Oblinger et al. [111] (b) Taking the ion energy $(11.8 \mathrm{eV})$ from (a) and the photoelectron cutoff energy of $0.85 \mathrm{eV}$, one calculates the $\mathrm{L}_{2}$ energy to $4.8 \mathrm{eV}$. 


\subsubsection{Ground-state processes}

The signal parts with long time constants $\tau_{3}$ and $\tau_{4}$ (reflected in the tails of the two fragment ion signals, Fig. 4.3.3) are already very weak, for both fragments by $2-3$ orders of magnitude weaker than from $\mathrm{L}_{2}$ (Table 4.3.1, Fig. 4.3.3). This indicates that ionization from $\mathrm{L}_{3}$ and $\mathrm{L}_{4}$ requires $1-2$ more photons for ionization than from $L_{2}$. (This conclusion is supported by the probe-intensity dependence of the $\mathrm{C}_{2} \mathrm{D}_{2}{ }^{+}$and $\mathrm{C}_{2} \mathrm{D}_{3}{ }^{+}$signals; the latter is illustrated in Fig. 4.3.3b. The evidence from the nondeuterated signals is not so clear however.) So, $\tau_{3}$ and $\tau_{4}$ are probably time constants of reactions in the hot ground state. Several reactions are known from hot $\mathrm{C}_{2} \mathrm{H}_{4}$ following internal conversion $[101,102]$ :

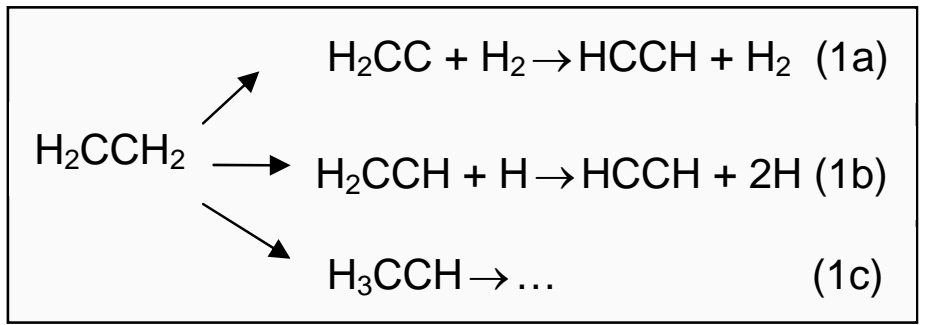

Scheme 1. Ground-state reactions of hot ethylene.

The branched first step in this scheme can give rise to only one observable time constant (inverse of the sum of the three rate constants), which would be the lifetime of hot ethylene. The subsequent - exothermic - step in (1a) would be too fast to be observed after the slow strongly endothermic - first step. While the second step in (1b) (less endothermic than the first) is energetically not accessible at $193 \mathrm{~nm}$, RRKM calculations predict that it is $8-12$ times faster than the first with the excess energy available with $157 \mathrm{~nm}(7.9 \mathrm{eV})$ excitation [112]. This is probably similar with our $162 \mathrm{~nm}(7.65 \mathrm{eV})$, so that this step will not be observed either. Similar considerations apply to the third line, since $\mathrm{CH}_{3} \mathrm{CH}$ decays primarily back to ethylene faster than it is formed. So only one of the two time constants $\tau_{3}$ and $\tau_{4}$ can be explained by Scheme 1 .

Here one should recall the possibility that $\mathrm{CH}_{3} \mathrm{CH}$ is also formed directly from the $\mathrm{S}_{1} / \mathrm{S}_{0} \mathrm{CI}$ (apart from ethylene, reaction (1c)). If this is the case, this path is much faster (17 fs) and kinetically independent from reaction (1c). Ethylidene rearranges rapidly to ethylene [113] in a nearly barrierless $[114,115]$ process; but $\mathrm{H}_{2}$ and probably $\mathrm{H}$ elimination from it is also observed (Scheme 2) [114]. This means that dissociation is competitive with rearrangement. 


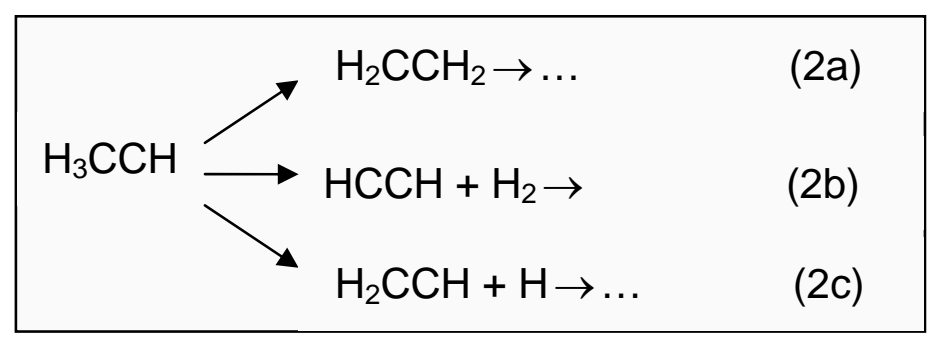

Scheme 2. Ground-state reactions of hot ethylidene.

The three reactions will again give rise to only one observable time constant. It is independent of that characterizing the reactions (1). We conclude that already the fact that two groundstate time constants $\left(\tau_{3}\right.$ and $\left.\tau_{4}\right)$ are observed points to formation of ethylidene directly from the CI, independently of the path (1c). Reaction (2a) is strongly exothermic (by $\approx 3 \mathrm{eV}$ ) [116] and barrierless or nearly so [114]. We can expect that it is faster than the endothermic reactions (1). This is confirmed by RRKM calculations, which predict rates of $(6-8.5) \times 10^{12} \mathrm{~s}^{-1}$ for it [112]. Therefore we assign $\tau_{3}$ to the lifetime of hot $\mathrm{CH}_{3} \mathrm{CH}$ (set of reactions (2)) and $\tau_{4}$ to the lifetime of hot $\mathrm{C}_{2} \mathrm{H}_{4}$ (set of reactions (1)).

The above assignment is further supported by the fact that the longer time constant $\left(\tau_{4}\right)$ is only contained in the $\mathrm{C}_{2} \mathrm{H}_{3}{ }^{+}$signal but not in the $\mathrm{C}_{2} \mathrm{H}_{2}{ }^{+}$signal (Fig. 4.3.3). This point shall be explained by Fig. 4.4.1.3.1.

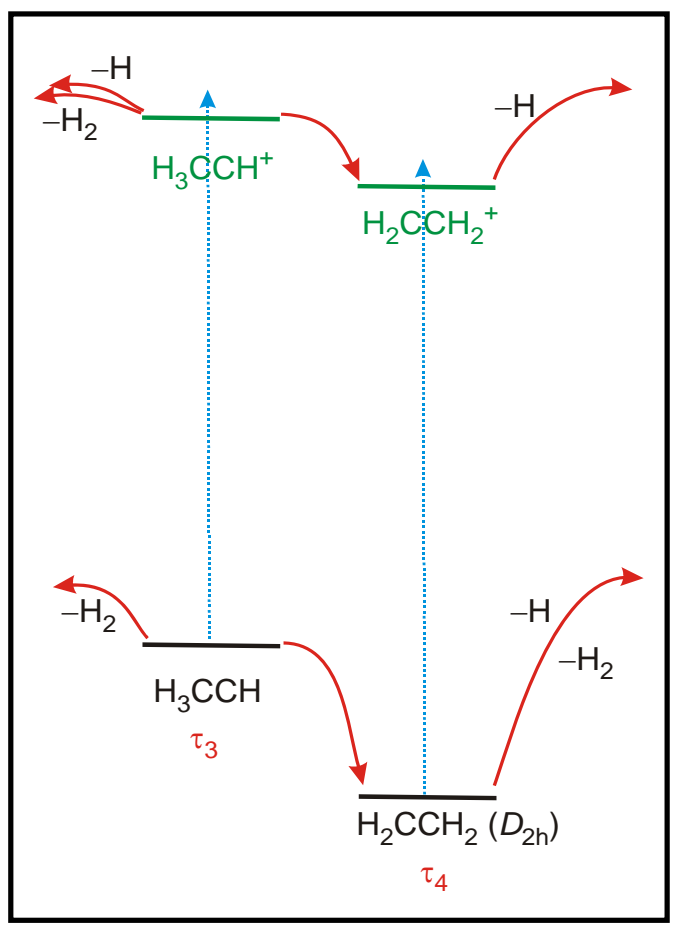

Fig. 4.4.1.3.1 Fragmentation in the ion is slightly different from that in the neutral molecule: $\mathrm{H}_{2}$ elimination takes place only from $\mathrm{H}_{3} \mathrm{CCH}^{+}$. The lifetime $\left(\tau_{4}\right)$ of hot ethylene will therefore not appear in the $\mathrm{C}_{2} \mathrm{H}_{2}^{+}$signal. 
It is generally accepted that $\mathrm{H}_{2}$ elimination from the $\mathrm{C}_{2} \mathrm{H}_{4}{ }^{+}$ion takes place only after hydrogen migration, i.e. from the ethylidene ion (left-hand side in Fig. 4.4.1.3.1) [110, 117]. Hence vertical ionization of hot $\mathrm{CH}_{3} \mathrm{CH}$ will reveal its short lifetime $\left(\tau_{3}\right)$ in the $\mathrm{C}_{2} \mathrm{H}_{2}{ }^{+}$ion (also in the $\mathrm{C}_{2} \mathrm{H}_{3}{ }^{+}$ion, see Fig. 4.4.1.3.1), whereas from ethylene in its hot ground state (lifetime $\tau_{4}$ ) vertical ionization and subsequent fragmentation will primarily lead to $\mathrm{C}_{2} \mathrm{H}_{3}{ }^{+}$, so that $\tau_{4}$ will be detected only in this signal, as is indeed the case. (Such a preferential elimination of $\mathrm{H}$ over that of $\mathrm{H}_{2}$ was also found, when $\mathrm{C}_{2} \mathrm{H}_{4}{ }^{+}$was generated as $\mathrm{H}_{2} \mathrm{CCH}_{2}{ }^{+}$with $\mathrm{D}_{2 \mathrm{~h}}$ structure by multiphoton ionization that was two-photon resonant with Rydberg states [118].) This also means that the ionic dissociation is kinetically controlled; if it were thermodynamically controlled i.e. only by the excess energy, independent of which location on the potential it starts from both channels would be equally probable, because the appearance potentials of the ions $\mathrm{C}_{2} \mathrm{H}_{3}{ }^{+}$ $(13.22 \mathrm{eV})$ and $\mathrm{C}_{2} \mathrm{H}_{2}^{+}(13.14 \mathrm{eV})$ are practically identical [119]. Also our observation of different kinetics of the $\mathrm{C}_{2} \mathrm{H}_{2}{ }^{+}$and $\mathrm{C}_{2} \mathrm{H}_{3}{ }^{+}$signals points to kinetic control of $\mathrm{C}_{2} \mathrm{H}_{4}{ }^{+}$dissociation.

The $\mathrm{D}$ isotope effect on $\tau_{4}$ is larger (2.2) than for a barrierless hydrogen motion (1.41) but probably consistent with the assignment to the endothermic $\mathrm{H}$ elimination. Also the $\mathrm{D}$ effect on $\tau_{3}(2.67)$ indicates an activated process. Perhaps this is because the (endothermic) $\mathrm{H}_{2}$ elimination contributes to $\tau_{3}$. It indicates that the ethylidene $\rightarrow$ ethylene rearrangement by $\mathrm{H}$ migration is not completely barrierless either, although some calculations predict that [114].

With the given interpretation, all the hot ground-state products (ethylene and ethylidene) will eventually end in acetylene (equations (1) and (2)), which will still have some excess energy. It is well conceivable that this end product was not detectable (via e.g. a long-lived pedestal in $\mathrm{C}_{2} \mathrm{H}_{2}{ }^{+}$signal), because (1) it has an even higher ionization energy $(11.4 \mathrm{eV})$ than ethylene $(10.5 \mathrm{eV})$ [120], so that one more photon (altogether 8$)$ would be needed for ionization, and (2) with the available excess energy it would fragment after ionization, so that its signature would only appear in lower-mass fragment ions that were not investigated. It is therefore not surprising why the $\mathrm{C}_{2} \mathrm{H}_{3}{ }^{+}$and $\mathrm{C}_{2} \mathrm{H}_{2}{ }^{+}$signals both decay to 0 .

By Fig. 4.4.1.3.2, the complete path of the molecule from the Franck-Condon region down to the ground state is summarized. The Rydberg part (see Fig. 4.2.3) is omitted from this figure. As already said, the $\mathrm{L}_{1}$ window (departure from the Franck-Condon region) reaches down to perhaps not far from the $\mathrm{V}-\mathrm{Z}$ intersection. In this region the $\mathrm{L}_{2}$ window begins, reaching as far as the $\mathrm{S}_{1} / \mathrm{S}_{0} \mathrm{CI}$. From the observation of the reactions of ethylidene, we conclude that this 
CI is displaced from the perpendicular $\left(D_{2 \mathrm{~d}}\right)$ minimum in a direction involving $\mathrm{H}$ migration (outside the drawing plane of Fig. 4.4.1.3.2). From the CI the path branches (1) to completion of this migration involving formation of $\mathrm{CH}_{3} \mathrm{CH}$ and (2) on returning the hydrogen to the original carbon, to planar $\left(D_{2 \mathrm{~h}}\right)$ ethylene with $\varphi=0$ or $180^{\circ}$.

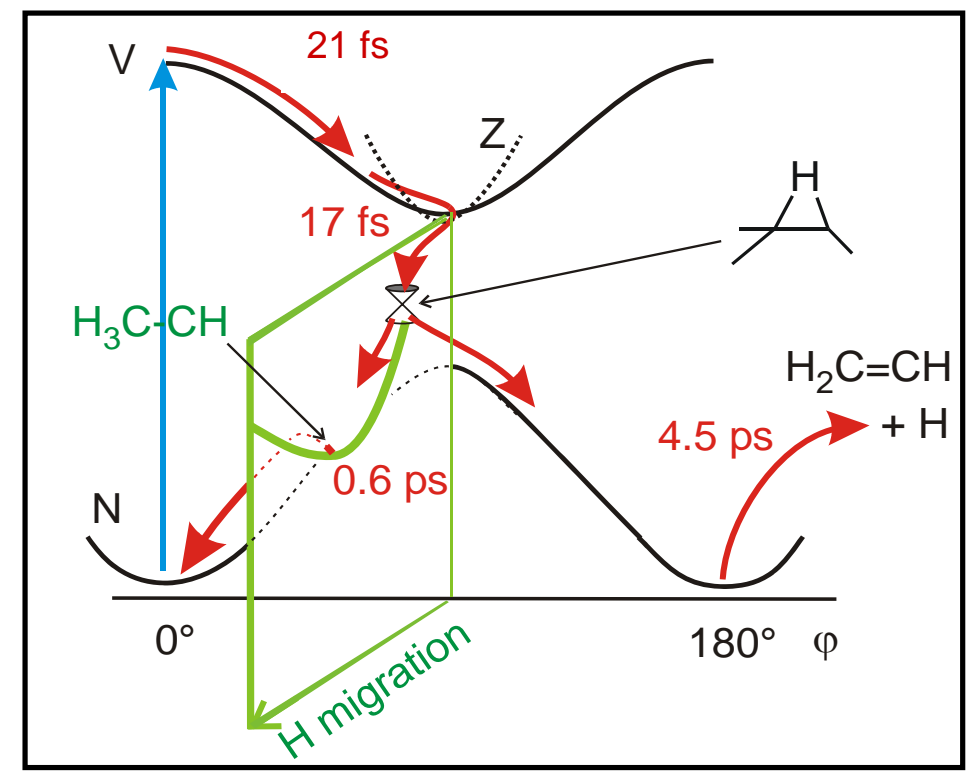

Fig. 4.4.1.3.2 Potentials and dynamics of $\mathrm{C}_{2} \mathrm{H}_{4}$ (for the time constants of $\mathrm{C}_{2} \mathrm{D}_{4}$, see Table 4.3.1), indicating also hydrogen migration (perpendicular to the drawing plane) and the displacement of the $\mathrm{CI}$ into this direction. The path starting in the Rydberg state is omitted. The two ground-state reactions are also identified. The pyramidalization of one $\mathrm{CH}_{2}$ group, predicted theoretically [92, 97, 99, 106, 107, 121-125], is not indicated. As argued in sec. 4.4.2, the turn to a new direction (to $\mathrm{H}$ migration and pyramidalization) does not occur so sharply (as indicated) in the perpendicular minimum but begins earlier and more smoothly.

\subsubsection{Results of spectroscopic relevance}

The UV absorption spectra of $\mathrm{C}_{2} \mathrm{H}_{4}$ and $\mathrm{C}_{2} \mathrm{D}_{4}$ have not yet been fully interpreted mainly because of the diffuseness of their vibrational structure, particularly in the case of the nondeuterated species. The broadening caused by the short lifetimes ( 21 and $24 \mathrm{fs}$ ) is $230 \mathrm{~cm}^{-1}$ and $200 \mathrm{~cm}^{-1}$, respectively for $\mathrm{C}_{2} \mathrm{H}_{4}$ and $\mathrm{C}_{2} \mathrm{D}_{4}$ and can be compared with the observed width of $400 \mathrm{~cm}^{-1}$ of the vibrational peaks near $180 \mathrm{~nm}$ in the spectrum of $\mathrm{C}_{2} \mathrm{H}_{4}$ (Fig. 4.2.2). A certain spectral congestion seems to contribute to the widths. In the region of $162 \mathrm{~nm}$ the maximum of the $\pi \pi^{*}$ absorption is covered by the $\pi 3 s$ Rydberg band, with its pronounced structure, and it is believed [85] to be a continuum. On the other hand, a clear vibrational progression is revealed in this wavelength region by the spectra in condensed phase [126128], in which Rydberg transitions are broadened and displaced to higher energies; however, the corresponding spectra of $\mathrm{C}_{2} \mathrm{D}_{4}$ are structureless [127]. In the long-wavelength end of the 
spectrum, there should be no congestion contributing to the broadening. In fact, the width found for a peak near $190 \mathrm{~nm}$ in $\mathrm{C}_{2} \mathrm{H}_{4}$ at $-78^{\circ}$ is $\approx 280 \mathrm{~cm}^{-1}$ [108]; this implies a lifetime of population $\geq 18$ fs near the perpendicular minimum. This is well consistent with our $\tau_{2}$ and the $\tau_{2}$ of Stert et al. [84] (which we attribute to departure from $\mathrm{S}_{1}$ ), but less consistent with the $\tau_{1} \approx 10 \mathrm{fs}$ measured in [84] with excitation at $198 \mathrm{~nm}$ (to an energy of $\approx 0.7 \mathrm{eV}$ above the perpendicular minimum).

There is agreement that the prominent progression of the absorption spectrum is due to $\mathrm{CC}$ twisting in a potential with minimum (in a cut in this direction) at $90^{\circ}$ and maximum at $0^{\circ}$. In the long-wavelength wing the spacing $\left(\approx 800 \mathrm{~cm}^{-1}\right)$ corresponds to one vibrational quantum near the minimum $[85,86,126]$. (Actually each vibrational level there is double, with wavefunctions with and without node below the barrier [129].) Near and beyond the spectral maximum, the structure corresponds to hindered free rotation and for symmetry reasons [129] the spacing ( $\approx 680 \mathrm{~cm}^{-1}$ in a cryogenic matrix or liquid) must correspond to two quanta. We find for the latter region (our observation window $\mathrm{L}_{1}$ ) 630 and $510 \mathrm{~cm}^{-1}$ for the two isotopomers. Probably the matrix contributes to the hindering potential, which raises the frequency there. Recent calculations of the spectrum via the wave-packet recurrence time found $680 \mathrm{~cm}^{-1}$ [100] or $650 \mathrm{~cm}^{-1}$ [105] for $\mathrm{C}_{2} \mathrm{H}_{4}$, very well consistent with our results. From the small deuterium effect (which was not possible to identify in the UV spectra and is not yet investigated theoretically), we conclude that the twist in this region is actually mixed with the $\mathrm{CC}$ stretch. This is in agreement with the calculations [92, 93, 100, 107], which predict that the $\mathrm{CC}$ bond is longer at $0^{\circ}$ than at $90^{\circ}$.

In the UV spectrum, a $\mathrm{C}=\mathrm{C}$ stretch vibrational quantum has only been observed with $\mathrm{C}_{2} \mathrm{D}_{4}$ [87] in its long-wavelength part of the UV spectrum. We assign the observed $\mathrm{C}_{2} \mathrm{H}_{4}$ vibration of wavenumber $1110 \mathrm{~cm}^{-1}$ in window $\mathrm{L}_{1}$ to $\mathrm{C}=\mathrm{C}$ stretch; the mixing of the $\mathrm{CC}$ twist and stretch vibrations implies that in the latter the isotope effect should be larger than expected for a pure $\mathrm{CC}$ stretch, as is in fact observed in $\mathrm{L}_{1}$, which is near the Franck-Condon region for 162-nm excitation. On the other hand, in $\mathrm{L}_{2}$ (the region from the perpendicular minimum to the $\mathrm{CI}$ ) the observed vibration is a pure $\mathrm{CC}$ stretch, according to the small isotope effect. Near $\varphi=90^{\circ}$ the torsional amplitude is in fact much smaller than above the barrier; on small torsional excursion, the $\mathrm{CC}$ distance is not expected to vary much, so that the two vibrations will not couple in that region. 
Finally, resonance Raman spectra suggest activity of more vibrations, including wagging (pyramidalization) and rocking (in plane bend) [88, 89]. In fact, such activity is expected, if the perpendicular minimum is actually a saddle point towards pyramidalization and $\mathrm{H}$ migration. Simulations of the UV spectrum can perhaps reveal them, if experimental numbers for the more evident vibrations are used. Our data might help in this respect. So far, the published simulations used widely different numbers for excited-state vibrations [130-136].

\subsubsection{Ultrafast dynamics and implications for the potentials}

The lifetime $\tau_{R}$ (80 and 115 fs for the two isotopomers) found for the Rydberg $\pi 3 s$ state, excited at $162 \mathrm{~nm}$ (excess energy $4400 \mathrm{~cm}^{-1}$ over the origin), is consistent with the time $\tau_{R} \geq 100$ fs for $\mathrm{C}_{2} \mathrm{D}_{4}$ deduced from a line width near the origin [87]. A slight shortening at higher energy is expected, if there is minor activation energy. In fact, the Rydberg potential has a minimum at a twist angle of $\varphi=25-30^{\circ}$ [94], rises by about $1.3 \mathrm{eV}$ at $\varphi=90^{\circ}$ (this value is taken over from the potential of the ion [110]) and at $\varphi \approx 35^{\circ}$ it intersects with the $\pi \pi^{*}\left(\mathrm{~B}_{1 \mathrm{u}}\right)$ state slightly above the $\pi 3 s\left(\mathrm{~B}_{3 \mathrm{u}}\right)$ minimum, which can explain a minor barrier (Fig. 4.2.3). Obviously the population can easily flow around the lower cone of the intersection (with a temporary distortion of $B_{3 \mathrm{u}} \times B_{1 \mathrm{u}}=b_{2 \mathrm{~g}}$ symmetry, i.e. a $\mathrm{CH}_{2}$ pyramidalization) to the $\pi \pi^{*}$ state and merge there with the population that has been excited directly to this state. This path seems evident, although in the spectroscopic literature (e.g. in [87, 137]) the leakage out from the Rydberg state was called predissociation.

The previous experimental studies of the ethylene dynamics had less time resolution and extracted the time constants by deconvolution. With excitation at $155 \mathrm{~nm}$ [81] or near $200 \mathrm{~nm}$ [82-84] they found $\tau_{1} \leq 40 \mathrm{fs}$ [81], $30 \mathrm{fs}$ [82], $20 \mathrm{fs}$ [83] and $10 \mathrm{fs}$ [84]. Our $\tau_{1}(21 \mathrm{fs})$ is within the error limit of the first three experiments and slightly out of this limit for the last one. According to $[106,138]$, not much different lifetimes for different excitation wavelengths is expected. According to [8] there are sometimes artifacts during the pump-probe overlapping times, if the absorption for the pump is very weak (such as for ethylene at $198 \mathrm{~nm}$, used in [84]). But it is also conceivable that the observation windows are different on probing the different locations on the potential.

$\tau_{1}$ was assigned as the time for leaving the Franck-Condon region of the $\pi \pi^{*}$ state and arriving in $\mathrm{L}_{2}$, which is the region (with population lifetime $\tau_{2}$ ) extending from around the per- 
pendicular minimum to the $\mathrm{S}_{1} / \mathrm{S}_{0} \mathrm{CI}$. Near a twist of $90^{\circ}$ one would expect that a zwitterionic state $\left(\mathrm{Z}\right.$ or $\pi^{*^{2}}$ ) is involved as well, reversibly taking over population from $\mathrm{V}\left(\pi \pi^{*}\right)$ and guiding it further towards the CI. We did not detect any $\pi \pi^{*} \rightarrow \pi^{*^{2}}(\mathrm{~V} \rightarrow \mathrm{Z})$ relaxation, probably because $\pi$ and $\pi^{*}$ orbitals become degenerate at $\varphi=90^{\circ}$, so that the ionization energies of the two states do not differ there; that is only the sum of the populations of the $\mathrm{V}$ and $\mathrm{Z}$ states is determined in our experiment.

One can define the excited-state lifetime as the sum $\tau_{1}+\tau_{2}(38$ and 45 fs for the two isotopomers), since after this time we only see ground-state signals. Such short times are only possible, if the path is continuous (involving no jump between surfaces), passing through a $\mathrm{CI}$, and involves no barrier. Without including a CI, a $\pi \pi^{*}$ lifetime of $\approx 1 \mathrm{~ns}$ is predicted [139, 140]. This is by orders of magnitude longer, implying that a CI is necessary for interpretation of the short times. (This was already pointed out in $[76,83]$.) In addition, it can be concluded that there cannot be a barrier before the CI. High-level calculations of the potentials suggest a minor barrier $[92,93,97,123,124]$, although this might be the case only along the shown cuts of the surfaces. Excited-state lifetimes calculated on the basis of these surfaces $[100,105$, $106,122,123,138]$ range from $\approx 100$ to $>250$ fs (summarized in [138]), whereas a method using semiempirical surfaces suggests 50 fs [141]. Only the latter value would be consistent with our $\tau_{1}+\tau_{2}$. In the case of the ab-initio surfaces [100,105, 106, 122, 123, 138], it seems that the initial geometrical relaxation (from $\varphi=0^{\circ}$ to $90^{\circ}$ and $\mathrm{CC}$ stretch) is fast enough and that the actual bottleneck in the calculations concerns the departure from $\mathrm{S}_{1}$. If the latter step is too slow, it also influences the population in the Franck-Condon region due to the reversibility of the V-Z relaxation. Perhaps some minor barrier before the $\mathrm{S}_{1} / \mathrm{S}_{0}$ CI causes the bottleneck in the calculations; in the ab initio methods $[100,105,106,138]$ the H-migration CI is not yet fully optimized, because symmetry constraints were used, whereas in the semiempirical work of Laino et al. [125] this is shown to be lowered in energy by dropping such constraints. Perhaps the following feature of the potentials might also play a role: the $17 \mathrm{fs}$ for $\tau_{2}$ seem too fast to be explained by the absence of a barrier alone; if the wave packet arrived at the perpendicular minimum and then had to search around to find the new direction towards the CI, this would probably take some time, perhaps connected with some oscillations in the preceding directions. (Trajectory calculations might show that.) If, however, there were already an early (although minor) acceleration to the new direction, this time would be saved. 
In fact, the electronic $\mathrm{V} \rightarrow \mathrm{Z}$ relaxation around the $\mathrm{CI}$ between the two surfaces involves an acceleration towards an asymmetric $\left(b_{1 u}\right)$ scissors coordinate $[106,142]$, which can be interpreted as an incipient $\mathrm{H}$ migration, i.e. a component of the direction towards the last CI. Such minor features may still be difficult to find out by high-level calculations. For clarity, this early and smooth turn to a new direction in the $\tau_{2}(17 \mathrm{fs})$ window is not indicated in Fig. 4.4.1.3.2. An early acceleration towards the last CI has already been previously suggested to explain short lifetimes $[34,74]$.

The small isotope effect (Table 4.3.1) observed in both $\tau_{1}$ and $\tau_{2}$ is remarkable. It means that during both phases not only hydrogen moves (e.g. along a twist coordinate) but also CC stretching or contraction is strongly involved. Hence the small isotope effects both in the lifetimes and the vibrations are likely to have the same origin: as explained above in the context of the vibrations, according to the calculations $[92,93,100,107]$ in the Franck-Condon region $\left(\mathrm{L}_{1}\right)$ the $\mathrm{CC}$ bond length initially expands, then towards $\varphi=90^{\circ}$ contracts again and does so further on the way to a H-migration CI. It would be desirable to check by calculating the dynamics also of $\mathrm{C}_{2} \mathrm{D}_{4}$ whether this feature is responsible for the small deuterium effect on the lifetimes and vibrational frequencies. In the previous experiment of Stert et al. (with lower time resolution), the deuterium effect on $\tau_{1}$ and $\tau_{2}$ was within their error limit [84]. For the Rydberg lifetime $\tau_{R}$ the $\mathrm{D}$ effect is normal, as expected for a motion along a pure twist via a nearly negligible barrier (see above). The D effect for the ground-state reactions has already been discussed.

The ground-state processes found indicate that one of the time constants $\left(\tau_{3}\right)$ represents the lifetime of the carbene $\mathrm{CH}_{3} \mathrm{CH}$ and the other $\left(\tau_{4}\right)$ that of hot ethylene, implying that both species are formed from the same $\mathrm{S}_{1} / \mathrm{S}_{0} \mathrm{CI}$ (Fig. 4.4.1.3.2). This means that the CI involves a partial H migration. More strictly speaking: the intersection-space region involving such a migration is easily accessible for the wave packet (probably supported by an early acceleration into this direction near the V/Z CI, see above), although perhaps a region without it (but with twist and pyramidalization, for instance) might be competitive. An H-migration CI was already suggested by Evleth and Sevin and by Ohmine [143, 144] and its importance pointed out once more by Freund and Klessinger [99], although they predicted a less symmetric structure. Later calculations preferred the one-sided pyramidalization (after a $90^{\circ}$ twist) $[97,107$, 122-124]. Recent high-level calculations point out the importance of H-migration for understanding hydrogen scrambling before dissociation $[92,106]$. However, they suggest that this 
CI (with imposed $C_{\mathrm{s}}$ symmetry) is not the minimum of the intersection space but a saddle point within it $[92,106]$, whereas at a less high level but without symmetry constraint, the twisted-pyramidalized CI is at higher energy than the H-migration CI [125]. The result that releasing the $C_{\mathrm{s}}$ symmetry constraint lowers the H-migration CI was already obtained earlier [99]. The strong ethylidene signal in our experiment indicates that the $\mathrm{H}$ migration channel is at least competitive, perhaps even dominant. (It is remarkable in this context that the semiempirical dynamics calculation of Granucci et al. [141] used a low-energy H-migration CI and obtained a realistic $\mathrm{S}_{1}$ lifetime, whereas the higher-level dynamics calculations - which did not have this CI or placed it at higher energy - obtained much longer lifetimes [100, 105, 106, $122,123,138]$.) But we suppose that the landscape of the $\mathrm{S}_{1}$ potential in the region between the perpendicular minimum and the last CI is rather flat in several directions.

\subsection{Conclusion}

The dynamics of the photochemical isomerization of ethylene after excitation of the $\pi \pi^{*}$ state (at $162 \mathrm{~nm}$ ) was investigated for the first time with very high time resolution; the pump and probe pulses were of duration about $10-12$ fs. Unlike previously, when short time constants have been extracted from measurements with longer pulses by the help of deconvolution, now five time constants (including the lifetime of the Rydberg state) and four vibrational frequencies could be resolved, helping to conclude on the reaction coordinates. Moreover, probing by ionization from the excited state, vibrations not revealed in the normal UV absorption spectra (due to diffuseness or overlap with the Rydberg band) could be detected in both the Franck-Condon region and the region before departure to the ground state, in both $\mathrm{C}_{2} \mathrm{H}_{4}$ and $\mathrm{C}_{2} \mathrm{D}_{4}$, since the Franck-Condon factors are different from those upon the $\mathrm{S}_{0} \rightarrow \mathrm{S}_{1}$ transition (e.g. $\mathrm{C}=\mathrm{C}$ stretch and the combination vibration).

The results suggest that the initial relaxation (window $\mathrm{L}_{1}$ with lifetime $\tau_{1}$ ) on the $\pi \pi^{*}(\mathrm{~V})$ surface involves $\mathrm{CC}$ torsion as far as to $90^{\circ}$, but from the beginning intimately coupled with stretching of the $\mathrm{CC}$ bond (as shown by the very small isotope effect on the torsion vibration), which initially expands and then contracts again. (At large torsion the V state probably equilibrates with the zwitterionic $(\mathrm{Z})$ state. However, our probing method cannot distinguish the $\mathrm{V}$ and Z states.) Later and before a complete twist of $\varphi=90^{\circ}$ is reached, the wave packet is gradually accelerated towards a new direction, which eventually leads to the conical intersection (CI) with the ground-state surface (window $\mathrm{L}_{2}$ with lifetime $\tau_{2}$ ). This additional distortion 
(besides a twist of $90^{\circ}$ and some $\mathrm{CC}$ stretching) not only involves pyramidalization of a $\mathrm{CH}_{2}$ group (according to theory), but also partial hydrogen migration. This conclusion was deduced from the observation of $\mathrm{CH}_{3} \mathrm{CH}$ (ethylidene with lifetime $\tau_{3}$ ) as the primary product besides hot ethylene (lifetime $\tau_{4}$ ). (The assignment was inferred from the detection of two ground-state reactions - time constants $\tau_{3}$ and $\tau_{4}-$, indicating that there are two primary products.) It is well known that the slope directions near the last CI decide on the fate of the reaction path and the type and yield of products. We use the observation of products to conclude on the slope directions. In quantum chemistry the H-migration CI is still under debate.

Finally, from our data conclusions concerning the photoproduct spectrum of olefins (carbene products compared to cis-trans and other isomerizations), as well as some properties of their bimolecular reactions in view of the excited-state lifetimes found here could be drawn (for details see [6]).

The spectroscopic results, which are now presented in this work, for both $\mathrm{C}_{2} \mathrm{H}_{4}$ and $\mathrm{C}_{2} \mathrm{D}_{4}$, have been searched for decades. It is also the first time that a complete path was experimentally determined for this molecule from the Franck-Condon region down to the ground state, with information on the different directions of motion and on geometrical structures such as that of the last conical intersection. The results might provide benchmarks for quantum chemical calculations of the potentials and dynamics of $\mathrm{C}_{2} \mathrm{H}_{4}$, an active field of research, and may contribute to the better understanding of olefin photochemistry. 


\section{5}

\section{The ring opening of cyclohexadiene}

\subsection{Experimental}

The molecule investigated in this chapter is cyclohexadiene $\left(\mathrm{C}_{6} \mathrm{H}_{8}\right)$, excited at $270 \mathrm{~nm}$, and the subsequent ring-opening, involving cleavage of the $\sigma \mathrm{CH}_{2}-\mathrm{CH}_{2}$ bond. This system has already been well studied by time-resolved spectroscopy and other experimental methods, as well as quantum chemically. It therefore seemed a good candidate to explore the potential of our improved time-resolution. The laser system and the experimental set-up used to generate the $810-\mathrm{nm}$ probe and the UV pump pulses for the time-resolved studies were described in Chapter 2. The 810-nm probe used has about 12 fs duration; the 270-nm 10-fs (pump) pulses are taken from the third-harmonic of the $810-\mathrm{nm}$ fundamental radiation, generated in a small steel cell (length $18 \mathrm{~mm}$ ) with pinholes, filled with $\operatorname{Ar}(\approx 200 \mathrm{mbar})$ and embedded in vacuum ( $<4$ mbar). The beam propagates further in vacuum through apertures (for differential pumping), via two dielectric mirrors (to isolate the $270 \mathrm{~nm}$, collimate and focus the beam to the mass spectrometer) and through the hole of a mirror, serving to merge it with the probe beam $(\approx 0.03 \mathrm{~mJ})$ (see Chapter 2, sec. 2.5). The duration (10 fs) of the $270-\mathrm{nm}$ pulses was determined by cross correlation with the fundamental in the mass spectrometer by ionizing Xe (1 $\mathrm{UV}+5 \mathrm{IR}$ photons, IE $=12.15 \mathrm{eV}$ ), from which also the time zero was obtained (the time when the maximum of the pump and probe pulses coincide).

\subsection{Background}

1,3-Cyclohexadiene (CHD) is a cyclic hydrocarbon consisting of six carbon and eight hydrogen atoms and two double bonds (Fig. 5.2.1). The molecular orbitals of CHD are delocalized over the whole ring; the $s p^{2}$ and $s p^{3}$ hybridized C orbitals (the former belonging to the carbon 
atoms that are involved in the double bonds and the latter to the ones that do not contribute to the double bonding) and the $1 s$ hydrogen orbitals make up the single bonds, which are relatively low in energy, constituting the $\sigma$-framework, while the four $2 p_{\mathrm{x}} \mathrm{C}$ atomic orbitals are combined to form four $\pi$ orbitals, higher in energy, namely (ignoring normalization and numerically exact coefficients) the $\pi_{1}=2 p_{\mathrm{x} 1}+2 p_{\mathrm{x} 2}+2 p_{\mathrm{x} 3}+2 p_{\mathrm{x} 4}, \pi_{2}=2 p_{\mathrm{x} 1}+2 p_{\mathrm{x} 2}-2 p_{\mathrm{x} 3}-2 p_{\mathrm{x} 4}$, $\pi_{3} *=2 p_{\mathrm{x} 1}-2 p_{\mathrm{x} 2}-2 p_{\mathrm{x} 3}+2 p_{\mathrm{x} 4}, \pi_{4} *=2 p_{\mathrm{x} 1}-2 p_{\mathrm{x} 2}+2 p_{\mathrm{x} 3}-2 p_{\mathrm{x} 4}$, two of which are filled with the four $2 p$ carbon electrons: $\pi_{1}^{2}, \pi_{2}^{2}$ (see e.g. [145]).

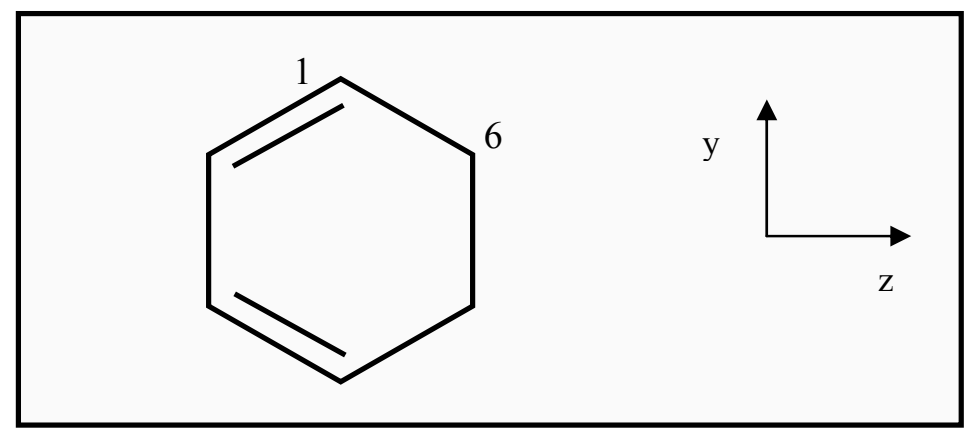

Fig. 5.2.1 Planar 1,3-cyclohexadiene (CHD).

Planar CHD belongs to the $C_{2 \mathrm{v}}$ symmetry point group and its ground state is the totally symmetric singlet $1{ }^{1} \mathrm{~A}_{1}$. Several studies have been carried out for the investigation of the CHD geometry in its ground state (e.g. [146-148]), pointing to a geometry slightly distorted from planarity, with one $\mathrm{CH}_{2}$ group slightly above and the other below the drawing plane of Fig. 5.2.1. The inversion barrier is about $1130 \mathrm{~cm}^{-1}$ [149]; the molecule then actually belongs to the $C_{2}$ symmetry point group, although the potential surface is still symmetric $\left(C_{2 \mathrm{v}}\right)$.

After absorption of light or on heating CHD can produce Z-hexatriene (HT) (Fig. 5.2.2). This electrocyclic ring-opening reaction of CHD (with its reverse, ring-closure of HT) constitutes a prototype of the so-called pericyclic rearrangements. Woodward and Hoffman [150] formulated some rules and demonstrated that such concerted reactions proceed only in a very stereospecific manner; they rationalized the rules by correlating the orbital symmetry of the reactants to that of the products, assuming that it is conserved throughout the course of the reaction. In other words, they pointed out that the symmetry of the highest occupied molecular orbital (HOMO) determines the stereochemistry of the product formed. Therefore the Woodward-Hoffmann (WH) rules can predict the stereochemical outcome of a pericyclic reaction, which is different depending on whether the latter is induced thermally or photochemically (and could, for example, be detected by different substituents). 
For the case of CHD the WH rules predict that the photoinduced ring opening and the production of HT proceed via a reaction path that preserves the $C_{2}$ symmetry of the molecule, that is, the two $\mathrm{CH}_{2}$ groups of the saturated part rotate in the same sense about a z-axis (parallel to the $\mathrm{C}_{2}$ axis) that passes through each carbon (conrotatory path); on the contrary, the thermally induced ring opening according to the WH rules follows the disrotatory path, with the two $\mathrm{CH}_{2}$ rotating in opposite directions, preserving the mirror plane (perpendicular to the molecular plane) as the symmetry element throughout the reaction.

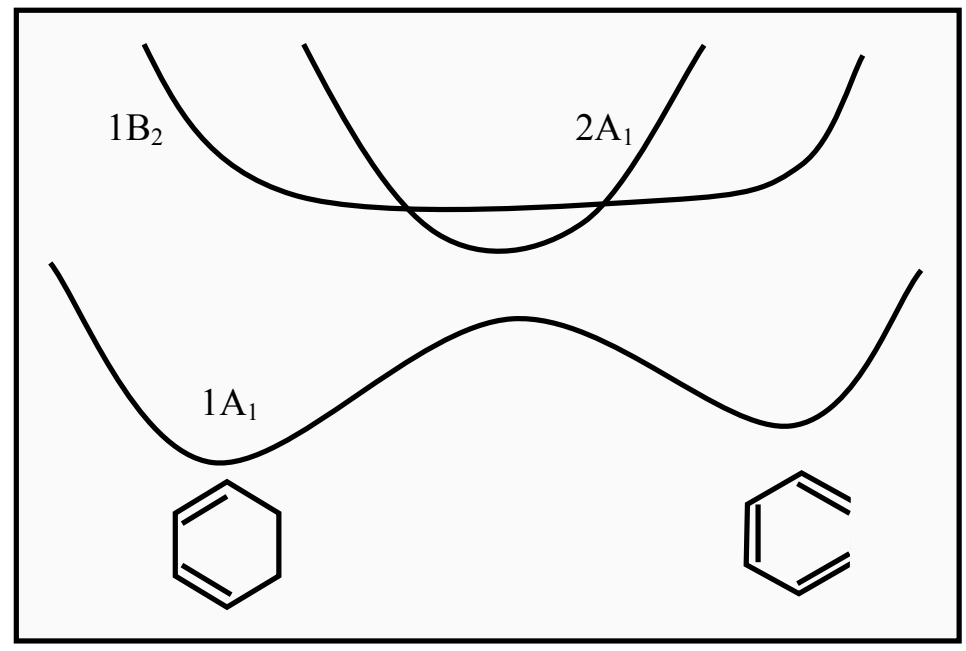

Fig. 5.2.2 van der Lugt and Oosterhoff type potential energy surfaces of CHD, for an excited-state allowed reaction, adapted from [151].

The WH model for the pericyclic reactions and its extension by van der Lugt and Oosterhoff [152] considers the correlation of the electronic states of the reactant (CHD) and the product (HT) molecules (Fig. 5.2.2) namely the ground (1 $\left.\mathrm{A}_{1}\right)$, first excited $\left(1 \mathrm{~B}_{2}\right)$ and a twoelectron excited state $\left(2 \mathrm{~A}_{1}\right)$, along the coordinate that preserves the $C_{2}$ symmetry. They found that the spectroscopically accessible $1 \mathrm{~B}\left(\pi \pi^{*}\right)$ state crosses the optically dark $2 \mathrm{~A}\left(\pi^{*^{2}}\right)$ potential (which involves excitation of two $\pi$ electrons) very early in the reaction path and that on 2A a "well" is created, just above the barrier of the ground-state surface, resulting from an avoided crossing. This well or "perpendicular minimum" effectively collects population; subsequent radiationless decay leads to the 1A surface, with either CHD or HT being formed.

An important step forward was made by Olivucci et al., who found that the collection well has an outlet in the form of a conical intersection (CI) (Chapter 3), located outside the drawing plane of Fig. 5.2.2 [153]. The well together with the CI form a funnel that guides the wave packet on a continuous path (i.e. without jump) to the lower surface in an ultrashort time and 
provides a branching region (on the lower cone), where the fate of the reaction is decided. Ultrafast spectroscopy can investigate this region and can confirm or reveal more details (see below).

\subsection{Results and analysis}

For the dynamics of the ring opening the parent $\mathrm{C}_{6} \mathrm{H}_{8}{ }^{+}$(mass 80) and the fragment $\mathrm{C}_{6} \mathrm{H}_{7}^{+}$ (mass 79) ion yields are measured as functions of the time delay between the pump and the probe pulses (varied in steps of $3.3 \mathrm{fs}$ ), while the time zero is determined from the maximum of the $\mathrm{Xe}^{+}$signal. According to [34] these two masses are sufficient to monitor the wavepacket motion on the excited-state surfaces. The transient ion signals are simulated by the function described in Chapter 3 (derived by a rate-equation model for the population decay), which is a sum of exponentials convoluted with the instrumental function, the crosscorrelation function between the UV pump pulse at $270 \mathrm{~nm}$ and the $3^{\text {rd }}-4^{\text {th }}$ power of the probe-ionizing pulse at $810 \mathrm{~nm}$; the extracted time constants correspond to the lifetimes of the population on the locations $\mathrm{L}_{i}$. As in the case of ethylene, the periodic modulations of the signals are interpreted as a modulation of the ionization probability due to a vibration of the molecule on $\mathrm{L}_{i}$.

The time-dependent parent-ion signal is shown in Fig. 5.3.1.
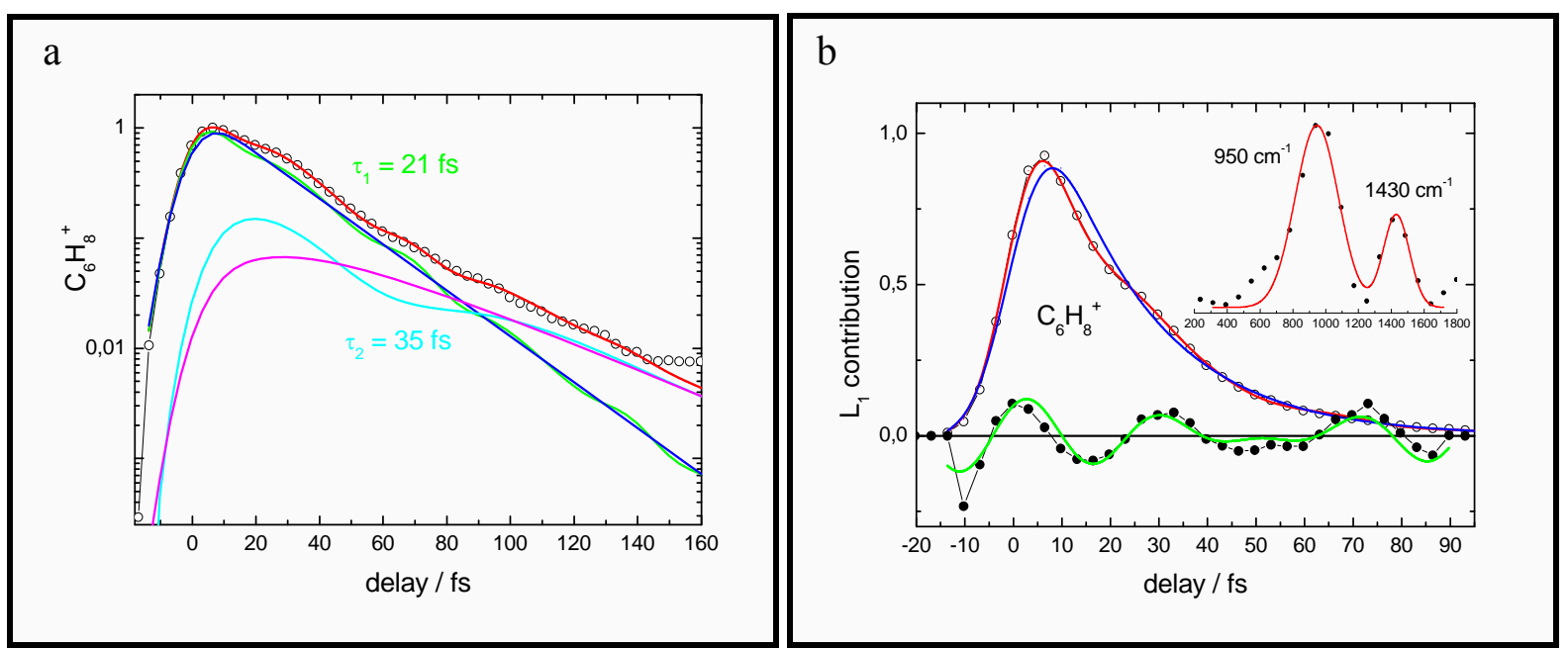

Fig. 5.3.1 (a) Transient parent ion signal simulated by a sum of two exponentials convoluted with the instrumental function (red curve), each level $\mathrm{L}_{i}$ being multiplied by a function of the type $f_{\mathrm{osc}}=1+\cos (2 \pi \mathrm{c} v t-\varphi)$; the contribution of the two levels $\mathrm{L}_{1}$ and $\mathrm{L}_{2}$ separately is also shown (with and without periodic modulation), (b) the contribution to the signal from $\mathrm{L}_{1}$ (measured data after subtraction of the light blue curve of Fig. 5.3.1a) 
together with the fit curve (with modulation, red line and without modulation, blue line); the oscillatory part with its Fourier transform are also shown.

Outside the pump-probe overlapping region $(70-150 \mathrm{fs})$ the signal decays exponentially with time constant $\tau_{2}=35 \mathrm{fs}$, showing also a modulation with wavenumber $v_{2}=300 \mathrm{~cm}^{-1}$. (As in ethylene, the modulation is represented by a factor $f_{\mathrm{osc}}=1+\cos (2 \pi \mathrm{c} v t-\varphi)$, by which the exponential decay is multiplied.) To extract also the earlier dynamics, $\tau_{2}$ and $v_{2}$ are fixed in a more complete fit function, which now contains two exponential decays $\left(\tau_{1}, \tau_{2}\right)$, convoluted with the instrumental function (and the $v_{2}$ modulation). The result is already a good simulation of the measured signal. The time constants $\tau_{1}, \tau_{2}$ reflect the lifetimes of the population in the two observation windows $\mathrm{L}_{1}$ and $\mathrm{L}_{2}$. Subtracting then the $\mathrm{L}_{2}$ contribution to the signal (including modulation, light blue line in Fig. 5.3.1a) and dividing the residue by the $\mathrm{L}_{1}$ decay (blue line in Fig. 5.3.1b) reveals the periodic function, $f_{\text {osc }}-1$, at the bottom, the Fourier transform of which gives the frequency of the modulated signal (inset). This value enters into the complete fit function, which is then used once again to simulate the measured data (Fig. 5.3.1a, red line) to get a final correction of the time constants $\tau_{i}$, the relative cross sections ${ }^{m} \sigma_{i}$ and the frequencies $v$ for all the observation windows. Fig. 5.3.1a and $\mathrm{b}$ show both the $\mathrm{L}_{1}$ and $\mathrm{L}_{2}$ contributions (lifetimes $\tau_{1}=21 \mathrm{fs}$ and $\tau_{2}=35 \mathrm{fs}$ ) and the contribution of $\mathrm{L}_{1}$ after subtraction of the $\mathrm{L}_{2}$ population from the measured data (as well as the simulation curves, see also caption in Fig. 5.3.1), respectively. In the same way the corresponding parameters of the observation window $\mathrm{L}_{2}$ are derived. The extracted frequencies, time constants and other fit parameters (also for the fragment signals) are compiled in Table 5.3.1.

The fragment-ion signal (Fig. 5.3.2) besides the contribution from a third location $\mathrm{L}_{3}$ (which does not contribute to the parent) contains also those from both $\mathrm{L}_{1}$ and $\mathrm{L}_{2}$. The latter time dependence (time constants and parameters of the modulation functions) are known from the parent ion and are subtracted. The residual signal (Fig. 5.3.2b) shows a decay ( $\tau_{3}=80 \mathrm{fs}$ ) with a modulation exhibiting two frequencies in the Fourier transform $\left(v_{3 \mathrm{a}}=140 \mathrm{~cm}^{-1}, v_{3 \mathrm{~b}}=\right.$ $\left.270 \mathrm{~cm}^{-1}\right)$; at early times ( $<100 \mathrm{fs}$ ) one can see that the signal also shows a high-frequency modulation with the two frequencies observed in $\mathrm{L}_{1}$; the structure also disappears within about $\tau_{1}$. We therefore ascribe it to a residue from $\mathrm{L}_{1}$, which is obviously not perfectly eliminated from the signal by our subtraction procedure. The error limits of the time constants and 
wave-numbers have not been evaluated. But they are believed to be near $\pm 5 \%$ as in previous experiments [34].
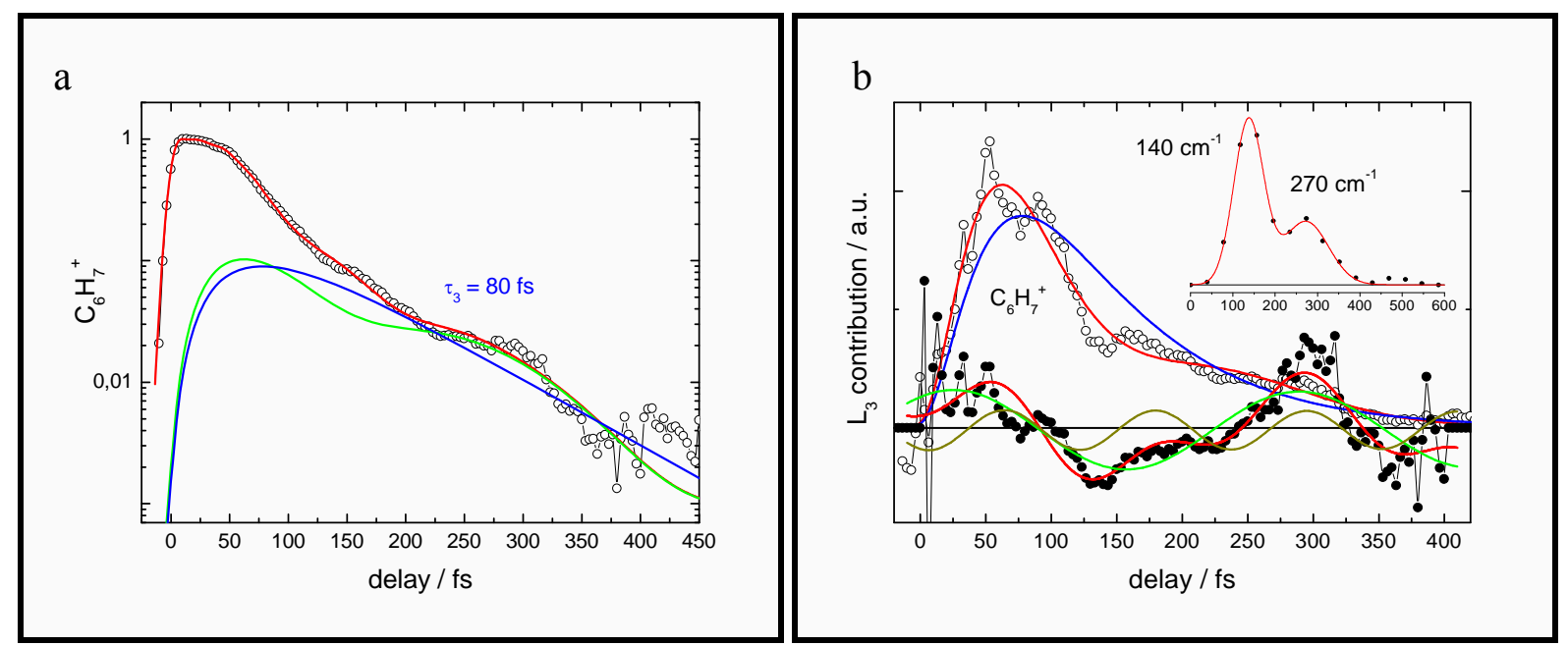

Fig. 5.3.2 (a) Transient fragment ion signal $\left(\mathrm{C}_{6} \mathrm{H}_{7}{ }^{+}\right)$simulated by a sum of three exponentials convoluted with the instrumental function, each of them multiplied by a function of the type $f_{\text {osc }}=1+A \cos (2 \pi \mathrm{c} v t-\varphi)$ (red curve); the contribution of the window $\mathrm{L}_{3}$ separately is also shown (with and without periodic modulation), (b) the contribution to the signal from the window $\mathrm{L}_{3}$ together with the fit curve (with modulation, red line and without modulation, blue line); the modulation part with the two oscillatory components (dark and light green) and its Fourier transform are also shown.

\begin{tabular}{|l|c|c|c|}
\hline $\mathrm{L}_{i} \quad\left(\mathbf{C}_{6} \mathbf{H}_{8}\right)$ & $\mathrm{L}_{1}$ & $\mathrm{~L}_{2}$ & $\mathrm{~L}_{3}$ \\
$\tau_{i}$ & $21 \mathrm{fs}$ & $35 \mathrm{fs}$ & $80 \mathrm{fs}$ \\
\hline $\mathrm{C}_{6} \mathrm{H}_{8}^{+}(80 \mathrm{u})$ & 1 & 0.10 & 0 \\
\hline $\mathrm{C}_{2} \mathrm{H}_{7}^{+}(79 \mathrm{u})$ & 1 & 0.92 & 0.16 \\
\hline$v / \mathrm{cm}^{-1}$ & 950,1430 & 300 & 140,270 \\
\hline
\end{tabular}

Table 5.3.1 Lifetimes $\tau_{i}$ of population in the locations $\mathrm{L}_{i}$, ionization cross-sections ${ }^{m} \sigma_{i}$ to produce an ion of mass $m\left(m\right.$ is indicated in parenthesis in column 1) from $\mathrm{L}_{i}$ and the oscillation frequencies from $\mathrm{L}_{i}$.

\subsection{Discussion}

The mechanism of the ring opening of CHD and the dynamics of the reaction have been extensively investigated both experimentally, (e.g. by transient ionization [34] and photoelectron spectroscopy [154]) and theoretically (e.g. $[151,155])$. With the better time resolution (13 fs, as compared to $130-150$ fs before) the reaction path is confirmed, in particular by the coherent oscillations that are now observed; they point to the reaction coordinates. With the help of 
the assignments discussed in [151] the identification of the steps along the reaction path follows in the next sections.

\subsubsection{Assignment of the lifetimes and oscillations}

Fig. 5.4.1.1 shows cuts of the potential surfaces along a $C_{2}$-preserving coordinate; on the lefthand side the measured time constants are assigned to three locations on these excited-state surfaces. The assignment is taken over from previous work [34, 151, 156]; it is based on the idea that the change of the electronic energy can be roughly estimated from the ionization probability and degree of fragmentation (see also ethylene, Chapter 4). That is, a lower-lying state is harder to ionize at $810 \mathrm{~nm}$, but, on the other hand, has vibrational excess energy (because the total energy is conserved) and such a hot molecule will give rise to a hot ion, which can then dissociate in the long time range (nanoseconds) before acceleration in the ion source.

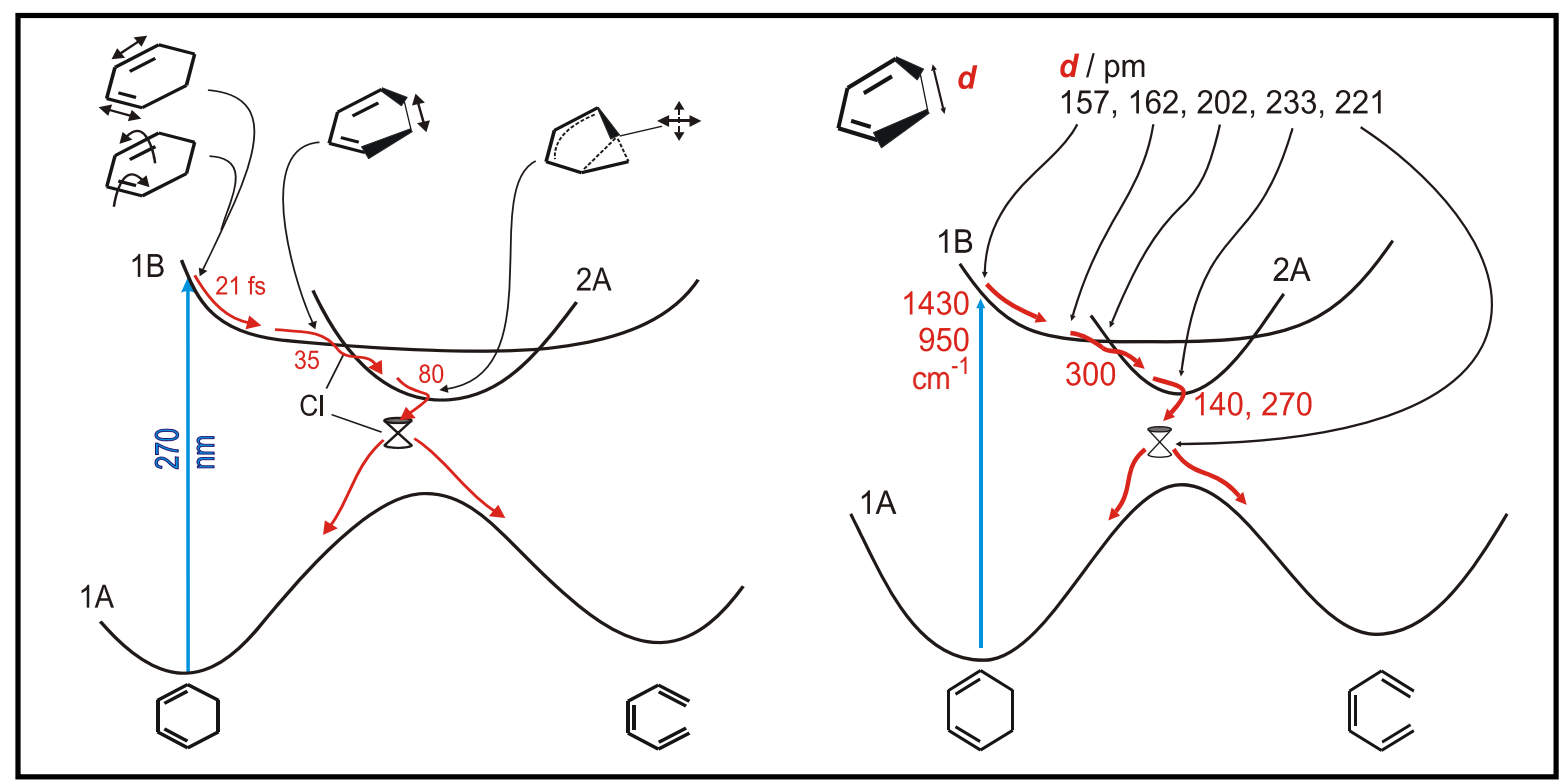

Fig. 5.4.1.1 Left: Potentials for CHD ring opening with assignment of the excited-state time constants and motion directions. The second conical intersection (CI) is out of the drawing plane, as is also the path around the first CI. Right: Correlation of wavenumbers of the ring vibrations with the calculated $\mathrm{CH}_{2}-\mathrm{CH}_{2}$ distance $d$ from [151].

The assignment was recently confirmed by time-resolved photoelectron spectroscopy [154]: the spectra show the $2 \mathrm{~B}$ state, from which the molecules relax within 55 fs (in perfect agreement with our $\tau_{1}+\tau_{2}=56 \mathrm{fs}$ ) towards a lower-lying state (2A) with lifetime $84 \mathrm{fs}$ (which agrees very well with our $\tau_{3}=80 \mathrm{fs}$ ); only one step on the $1 \mathrm{~B}$ surface was resolved with the long pulses in [154]. Moreover, these results agree well with the previously determined time constants in $[34,156]\left(\tau_{\text {old }}=10 \mathrm{fs}, \tau_{2 \text { old }}=43 \mathrm{fs}, \tau_{\text {3old }}=77 \mathrm{fs}\right)$, if the sum $\tau_{1}+\tau_{2}$ is compared 
(even if the individual lifetimes are compared, however, the agreement is still satisfactory within the standard deviation ( $\pm 5 \mathrm{fs}$ ) quoted in $[34,156]$ ); the reason for the small deviation is perhaps that the deconvolution method might be more accurate for the sum than for the individual times $[34,156]$. On the other hand, if the limit between the detection windows $\mathrm{L}_{1}$ and $\mathrm{L}_{2}$ (located in a region, where there is a sufficient change in the ionization energy (ionization probability)), is not very distinct (because the ion potential rises only gradually), it may be that the now shorter pulses with their 10 times broader coherent bandwidth require a larger change of the ionization energy, so that $\tau_{1}$ is lengthened on expense of $\tau_{2}$.

From the fluorescence quantum yield a lifetime of the emitting state of 10 fs was inferred in $[157,158]$ and it was suggested there that emission is stopped by depletion from the spectroscopic $1 \mathrm{~B}$ to the dark $2 \mathrm{~A}$ state. This is not compatible with the $\tau_{1}+\tau_{2}=56 \mathrm{fs}$. We suggest that fluorescence fades much earlier, already on the 1B surface, by a strong long-wavelength shift along the path. (The radiative rate depends on the emission wavelength $\lambda$ as $\lambda^{-3}$ [159].) In fact, the $\mathrm{S}_{0}$ potential is expected to rise steeply along the path in $\mathrm{L}_{2}$, which involves $\mathrm{CH}_{2}-\mathrm{CH}_{2}$ stretching (see below). One can conclude that the detection windows are not defined by the molecule alone but also by the probing process.

The vibrations excited in $\mathrm{L}_{1}$ are expected to belong to the Franck-Condon active ones and hence involve the bonds in the $\pi$ system, which change by excitation. An obvious assignment is the symmetric $\mathrm{C}=\mathrm{C}$ stretch and the $\mathrm{C}-\mathrm{C}$ stretch vibration for $v_{1 \mathrm{a}}=1430 \mathrm{~cm}^{-1}$ and $v_{1 \mathrm{~b}}=950$ $\mathrm{cm}^{-1}$, respectively (ground-state values 1579 and $945 \mathrm{~cm}^{-1}$, the latter strongly mixed with other vibrations $[149,160])$. In the UV absorption spectrum of CHD a weak modulation with four distinguishable peaks is detected (see Fig. 4 in [7] and [151]), converging from 1540 to $1360 \mathrm{~cm}^{-1}$ (average $1440 \mathrm{~cm}^{-1}$ ). The convergence is too rapid to be due to anharmonicity, but is characteristic of superposition of two or more progressions in the way demonstrated in [132]. The UV spectrum is much less well resolved than our Fourier transform. Hence the broadening in the former (see the simulations in [132]) is not only caused by the short lifetime ( $\tau_{1}=20 \mathrm{fs}$ would give rise to a width of only $250 \mathrm{~cm}^{-1}$ ) but also by activity of additional vibrations that are not active in ionization probing. A Franck-Condon activity of the $\mathrm{C}=\mathrm{C}$ vibration on ionization from the $1 \mathrm{~B}$ state was also found in the time-resolved photoelectron spectrum $\left(1350 \mathrm{~cm}^{-1}\right.$ in the ion) [154]. 
In $\mathrm{L}_{2}$ an oscillation with $v_{2}=300 \mathrm{~cm}^{-1}$ is observed. Such a low wavenumber can only be due to ring puckering (ring twisting) of the backbone; in the ground state these vibrations have wavenumbers of $199\left(v_{19}\right)$ and $506 \mathrm{~cm}^{-1}\left(v_{18}\right)[149,160]$, not including antisymmetric $(b$ in $C_{2}$ ) vibrations (which would appear only as overtones, if active at all).
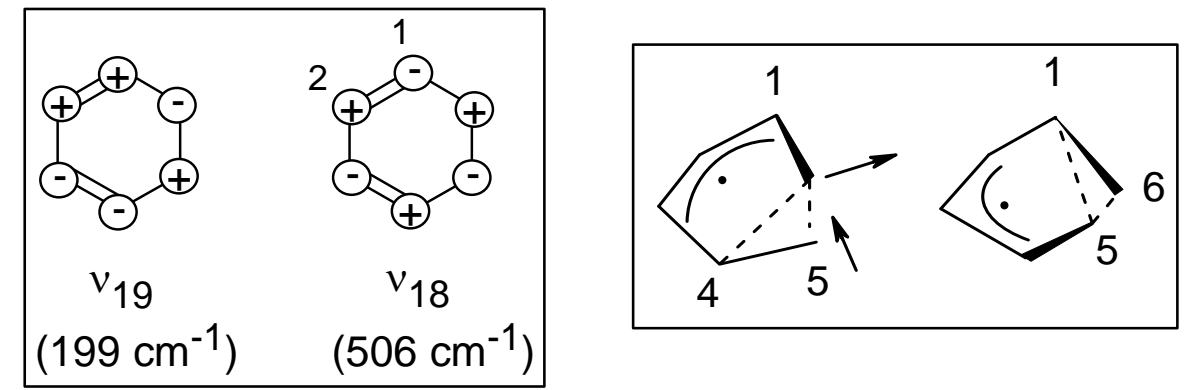

Scheme 1 Left: vibrations in $\mathrm{L}_{2}$ and $\mathrm{L}_{3}$ as discussed in the text. Right: the two symmetry-equivalent structures in the $2 \mathrm{~A}$ minima; the arrows indicate the coordinate connecting them, which is also the coordinate of the $140-\mathrm{cm}^{-1}$ vibration [7].

$v_{19}$ represents a twist of the $\mathrm{CH}_{2}-\mathrm{CH}_{2}$ group against the rest of the molecule, whereas $v_{18}$ can be described as a conrotatory twist of the two $\mathrm{C}=\mathrm{C}$ bonds. The observed $v_{2}$ cannot correspond to $v_{19}$, since this would imply a raise of frequency by $50 \%$ in $1 \mathrm{~B}$, which is quite unlikely. In particular also because resonance Raman spectra $[157,161]$ indicate an early acceleration into the $v_{18}$ direction but not towards $v_{19}$, we compare our $v_{2}$ in $1 \mathrm{~B}\left(\mathrm{~L}_{2}\right)$ with the $506 \mathrm{~cm}^{-1}$ in the ground state. The smaller wavenumber in $1 \mathrm{~B}$ indicates that the restoring force in this state is already strongly reduced along this coordinate. In fact the calculation of [151] for this vibration in the $1 \mathrm{~B}$ minimum (point "MEP(6)", which we identify with a point in our $\mathrm{L}_{2}$ ) reports a value of $245 \mathrm{~cm}^{-1}$, not too far from our experimental $v_{2}$.

As said above, location $\mathrm{L}_{3}$ is assigned to the $2 \mathrm{~A}$ state. According to the calculations (e.g. [151]), the 2A potential has two symmetry-equivalent very flat minima, in which the $C_{2}$ symmetry is broken (see Fig. 5 in [151]). The structures are shown on the right in Scheme 1: in one, C-atom 6 forms a weak three-electron three-centre bond with atoms 4 and 5, whereas in the other the three-centre bond connects atoms 1,5 and 6. The arrows indicate the coordinate $\left(\mathrm{p}^{*}-\mathrm{CI}\right)$ connecting the two minima via the flat $C_{2}$-symmetric saddle point (the traditional "pericyclic minimum" $\mathrm{p}^{*}$ ); it is hence the symmetry-breaking coordinate $\left(b\right.$ in $C_{2}$ ). Proceeding further along this direction beyond one of the two minima leads to the $2 \mathrm{~A} / 1 \mathrm{~A} \mathrm{CI}$. The main 
oscillation observed in $\mathrm{L}_{3}$ has a very small wavenumber $\left(v_{3 \mathrm{a}}=140 \mathrm{~cm}^{-1}\right)$, pointing to a small restoring force of the vibration and (or) movement of large masses; it hence seems to also correspond to some ring deformation (like $v_{2}$ ). An obvious candidate is a vibration along the symmetry-breaking coordinate $\left(\mathrm{p}^{*}-\mathrm{CI}\right)$ above, because the potential is very flat in this direction. In fact, its wavenumber has been calculated in the 2A minimum: Celani et al. report 135 $\mathrm{cm}^{-1}$ (Fig. 5 of [153]), whereas $204 \mathrm{~cm}^{-1}$ was found more recently [162]; in both cases it is the lowest frequency in this state. The small restoring force (flatness of the potential) is unique for the $\mathrm{p}^{*-}-\mathrm{CI}$ coordinate. Therefore we assign our $v_{3 \mathrm{a}}=140 \mathrm{~cm}^{-1}$ oscillation to a vibration in this direction.

However, this coordinate is antisymmetric (symmetry type $b$ ) in $C_{2}$. The molecule has enough excess energy to vibrate above the small barrier between the minima, so that the effective symmetry is $C_{2}$; but then only overtones $(\Delta \mathrm{v}=2,4 \ldots)$ should be observed [79] (because the ion is probably also $C_{2}$ symmetric). The lowest calculated frequency (derived from the curvature in the minimum within the harmonic oscillator approximation) would then be larger by a factor of $2-3$ than the observed one. However, above the barrier of a double-minimum potential a vibrational frequency usually drops by a factor of two; the observed $140 \mathrm{~cm}^{-1}$ would then correspond to a $\Delta \mathrm{v}=2$ overtone of the calculated vibration (whose frequency should actually then be lowered by this anharmonic effect) and the higher wavenumber $\left(v_{3 \mathrm{~b}}=\right.$ $\left.270 \mathrm{~cm}^{-1}\right)$ observed also in $\mathrm{L}_{3}$ may then be the next higher overtone $(\Delta \mathrm{v}=4)$. It would be unlikely that $v_{3 \mathrm{~b}}$ is just the same ring puckering as in $\mathrm{L}_{2}$ (wavenumber $v_{2}=300 \mathrm{~cm}^{-1}$ ), since the geometrical structure and bonding at the two locations are very different. Also optimal-control experiments [163] confirm that $v_{3 \mathrm{a}}$ is a vibration in a direction leading to the last CI, as explained in [7].

The high-frequency modulation (Fig. 5.3.2b) in the early part of the fragment ion signal has a frequency and a decay time coinciding with those of the $\mathrm{L}_{1}$ contribution to the parent ion (Fig. 5.3.1b). (The exact decay time is difficult to extract, because of the different exponential contributions to the fragment signal.) Therefore we attribute this modulation (CC stretch vibrations) to a residue from $\mathrm{L}_{1}$, which has not been perfectly eliminated by our subtraction procedure. If it belonged to $\mathrm{L}_{3}$, it should last over a time comparable to that of the $\mathrm{L}_{3}$ signal (Fig. 5.3.2b). 


\subsubsection{The reaction path}

After excitation the molecules are guided by the slopes of the potential, that is, by driving forces, which can change their direction repeatedly along the travelling path [34, 151]. Downslopes can also cause oscillations of the wave packet. Therefore the vibrational coordinate can provide information on the direction of the slope. As already pointed out in [151], the initial slope of every photochemical reaction is towards Franck-Condon (FC) active coordinates, which in the case of CHD involve the $\pi$ system. Only later, when the molecule is enough distorted to allow also interaction of $\sigma$ bonds with the $\pi$ system, the Woodward-Hoffmann (WH) rules are "activated" and the direction of motion changes (by modifying the $\sigma$ bond length, for instance) [151]. This rule is relatively general: it was also found in $\mathrm{H}$ migration in cycloheptatriene [164], for example, and plays an important role in cyclobutene ring opening, in which case it can explain deviations from the WH rules [165].

The FC active modes besides bond stretching of the $\pi$ system, involve also double-bond torsion [151]. The latter distorts all the backbone of the molecule, erecting also the $\mathrm{CH}_{2}-\mathrm{CH}_{2}$ bond (Fig. 5.4.1.1 left), so that after some travelling on the potentials the WH $(\sigma-\pi)$ interactions can set in. The more recent calculation by Tamura et al. finds the same path [155]. In $\mathrm{L}_{1}$ (FC region) the two $\mathrm{CC}$ stretch vibrations and in $\mathrm{L}_{2}$ a $\mathrm{C}=\mathrm{C}$ torsion $\left(v_{18}\right.$, Scheme 1$)$ are observed. Probably due to the long period (120 fs) the latter cannot be observed in the shortlived (21 fs) $\mathrm{L}_{1}$ window, but is certainly excited there and not stimulated later, because according to the calculations the lower part of the $1 \mathrm{~B}$ surface before the intersection with $2 \mathrm{~A}$ (i.e. location $\mathrm{L}_{2}$ ) is energetically very flat and so, as towards the other coordinates, there is no slope towards $v_{18}$ either. The fact that $v_{18}$ is actually $\mathrm{FC}$ active is confirmed by the resonance Raman spectrum [157, 161]. The initial motion and acceleration directions are therefore confirmed by the oscillations found here.

It is worth noting that the conrotatory initial acceleration (along $v_{18}, a$ symmetry) follows from the fact that $v_{18}$ is totally symmetric in $C_{2}$ and thus can be FC active and it does not mean that the $\mathrm{WH}$ rules are active already in the $\mathrm{FC}$ region; the corresponding disrotatory combination of the $\mathrm{C}=\mathrm{C}$ torsions $\left(v_{36}, b\right.$ symmetry [149]) is antisymmetric and $\mathrm{FC}$ inactive. (If CHD were planar, symmetry group $C_{2 v}$, also $v_{18}$ would by antisymmetric, symmetry type $a_{2}$.) The conrotatory twist $\left(v_{18}\right)$ of the $\mathrm{C}=\mathrm{C}$ bonds also implies a torsion of the $\mathrm{C}-\mathrm{C}$ bond be- 
tween them; this is just the coordinate $\varphi$, identified in the calculations of de Vivie-Riedle et al. as the direction of initial motion [166].

The low-frequency oscillation $\left(140 \mathrm{~cm}^{-1}\right.$ and its overtone $\left.270 \mathrm{~cm}^{-1}\right)$ in $\mathrm{L}_{3}(2 \mathrm{~A}$ state) was assigned above to an (overtone) vibration along the minimum-energy path, i.e. along the line $\mathrm{p}^{*}-\mathrm{CI}$ (antisymmetric in $C_{2}$ ) connecting the $C_{2}$-symmetric stationary point of $2 \mathrm{~A}$ ("pericyclic minimum" $\mathrm{p}^{*}$ ) with the $2 \mathrm{~A}$ minima and the 2A/1A CIs (Scheme 1). Since this region of the potential is very flat $[151,155]$, the vibration must have been stimulated before (but not excited in the FC region, as it is antisymmetric and also because it very much involves the former $\mathrm{CH}_{2}-\mathrm{CH}_{2}$ bond - see Scheme 1 -, which should not initially be affected by the excitation). In addition, it was pointed out that $1 \mathrm{~B} \rightarrow 2 \mathrm{~A}$ relaxation requires an antisymmetric ( $b$ type) distortion, so that the wave packet circumvents the 1B/2A CI around its lower cone; the calculation says that this deflection is just in the $\mathrm{p}^{*}-\mathrm{CI}$ direction $[151,155]$. The observation of an oscillation in the $\mathrm{p}^{*}-\mathrm{CI}$ direction now provides a neat experimental confirmation of this antisymmetric deflection near the first CI.

Fig. 5.4.2.1 it is illustrates, how the oscillations $v_{2}$ and $v_{3}$ are stimulated: $v_{2}\left(v_{18}\right)$ is excited in the FC region. Only a small part of the wave packet completes a full period, whereas the dominant part falls down to the $2 \mathrm{~A}$ surface and, around the $1 \mathrm{~B} / 2 \mathrm{~A} \mathrm{CI}$, is accelerated to an antisymmetric direction, which eventually leads to the $v_{3}$ vibration along the $\mathrm{p}^{*}-\mathrm{CI}$ direction over the double minimum. Also in this case, only a small part of the wave packet performs a full period, whereas most of it passes through the CI region down to the ground-state surface.

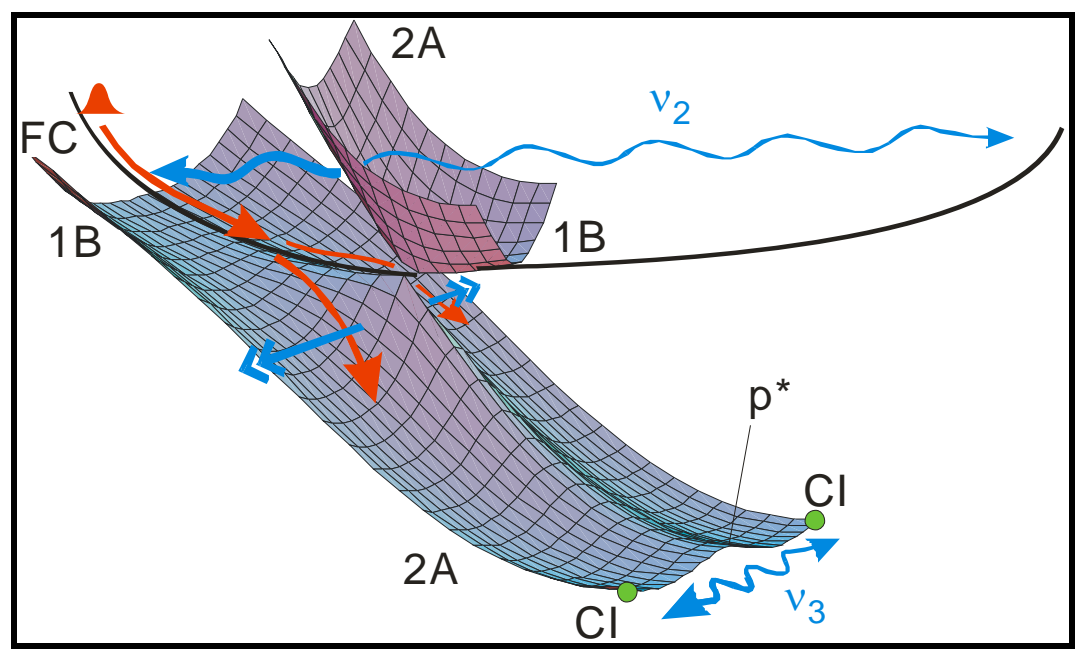

Fig. 5.4.2.1 Surfaces and illustrations of acceleration forces (double arrows) and directions of vibrations (wavy lines; actually the indicated ranges only comprise a single period). For a calculated surface, see [151]. Most of 
the wave packet falls down from the 1B to the lower 2A surface near the first CI; a small fraction remains, however, on 1B surface and completes an oscillation (thin wavy line). For the final CI, only the two (symmetryequivalent) positions are indicated. Vibrations in the Franck-Condon (FC) region follow different coordinates $(\mathrm{C}=\mathrm{C}$ and $\mathrm{C}-\mathrm{C}$ stretch $)$ and therefore cannot be indicated in this figure.

An early acceleration (near the first CI) towards the last CI was also invoked in [151] to rationalize the short $\tau_{3}$. In fact, on a flat potential such as from $\mathrm{p} *$ to the $\mathrm{CI}$, in the absence of a strong acceleration, the wave packet would normally find the outlet (the $2 \mathrm{~A} / 1 \mathrm{~A} \mathrm{CI}$ ) only by kind of a diffusive process, which would be slow. In contrast, the fastest conceivable process would be a ballistic motion from the initially symmetric structure to the antisymmetrically distorted turning point; starting from $\mathrm{p}^{*}$, the wave packet could then reach the CI region (which is near the turning point) in a quarter of a period of the corresponding vibration. This time would be $120 \mathrm{fs}$ for the $140 \mathrm{~cm}^{-1}$ vibration (which we assign to an overtone), longer than $\tau_{3}=80 \mathrm{fs}$. However, the acceleration in this direction is much faster near the first CI, because the negative curvature on the lower cone of this CI is very much larger than at the stationary point $\mathrm{p}^{*}$. Hence in this interpretation a ballistic path of the wave packet in an antisymmetric direction, leading eventually to the outlet to the ground state, already begins in the surrounding of the first CI.

An alternative or supplementary mechanism to explain the short $\tau_{3}$ was also suggested in [151]: "The 2A/1A CI" actually means the energy minimum of the $2 \mathrm{~A} / 1 \mathrm{~A}$ intersection space (IS). The IS extends further to molecular structures closer to $C_{2}$-symmetric geometries. (A point in the IS with $C_{2}$-symmetry lies, however, higher than our excitation energy $[151,155]$.) If energetically reachable parts of the IS are, e.g. by $30 \%$ closer to a $C_{2}$-symmetric structure, a ballistic trajectory could arrive at it in about 30\% less time than in 120 fs, i.e. in about the measured $\tau_{3}$. However, even in this case an early and rapid acceleration is necessary to trigger a ballistic motion. It seems likely that this acceleration leads to a region of the IS, which involves asymmetric distortion of the molecule and is energetically easily accessible, although not the very minimum of the IS, as already suggested in [151]. The time for departure from the 2A surface was calculated by quantum dynamics to $130-180$ fs after leaving the FC region [155]. This is in the range of our $\tau_{1}+\tau_{2}+\tau_{3}=136 \mathrm{fs}$.

A more or less ballistic path is only conceivable if any barriers are negligible compared to the available excess energy. This is practically consistent with the calculations (an energy dif- 
ference of $\approx 0.09 \mathrm{eV}$ was found between $2 \mathrm{~A}$ minimum and the last CI [155]), but can primarily be inferred from the short time constants and the fact that they are the same in a cold supersonic beam [154]. A barrier before the last CI might arise, if extension of the $\pi$ system (like in photochromic substances) lowers the spectroscopic state much more than the $2 \mathrm{~A}$ state and hence the 2A/1A CI; activation energies were in fact calculated for such systems [167] and relatively slow ring-opening and closing times were found $[168,169]$.

An instructive correlation of the wavenumbers of the low-frequency vibrations with the calculated [151] distances $d$ of the two $\mathrm{CH}_{2}$ groups is possible, even without detailed knowledge of the nature of $v_{3}$ (Fig. 5.4.1.1). The low frequencies indicate that these vibrations must be delocalized over the ring and have a small restoring force such as twisting or bending. The lowered wavenumber of $v_{2}\left(300 \mathrm{~cm}^{-1}, 59 \%\right.$ of the ground-state value of $\left.v_{18}=506 \mathrm{~cm}^{-1}\right)$ indicates that the ring is already weakened (restoring force $33 \%$ of that in $\mathrm{S}_{0}$ ), consistent with the calculated beginning of $d$ lengthening near the first CI $\left(\mathrm{L}_{2}\right)$. A more drastic decrease of the wavenumber (factor of 2; or 4, if it is an overtone) and force constant (factor of 4 or 16) occurs on arrival at the 2A minimum. This is consistent with the very long calculated $d$ and the only weak interaction of one $\mathrm{CH}_{2}$ group with the other end of the ring. The wavenumbers hence demonstrate (in agreement with theory) that the ring begins to open near the1B/2A CI (near the $1 \mathrm{~B}$ minimum) but advances much more on the $2 \mathrm{~A}$ surface. However, the reaction is not completed in the excited state; otherwise a ring vibration would not exist any more.

For completeness, one should consider here the possibility that - despite the calculations the ring would already be fully open in the $2 \mathrm{~A}$ state and the $140 \mathrm{~cm}^{-1}$ would be a hexatriene (HT) vibration. Indeed, in the ground state, Z-hexatriene has vibrations of such low wavenumbers [170]. They correspond to hindered free rotation around the $\mathrm{C}-\mathrm{C}$ bonds and $\mathrm{CCC}$ bending. However, neither of them would be Franck-Condon active in the probing transition, since the respective angles in the ion would not differ (much) from those in the neutral molecule. Hence these HT vibrations would not be observed and the observed oscillation must be due to the intermediate structure discussed above. Furthermore, it was shown in [34] by means of an ionic resonance that a structure corresponding to Z-hexatriene is only reached after arrival on the $\mathrm{S}_{0}$ surface. 


\subsection{Conclusions}

For photochemical reactions, Woodward and Hoffmann [150] only considered the orbitals and the spectroscopic state (1B), that is the state resulting from excitation of one electron from the highest occupied (HOMO) to the lowest unoccupied (LUMO) molecular orbital. Whereas in this way one could rationalize, for instance, the ring opening of cyclobutene to a diene, the backward reaction would require an additional energy of $>2 \mathrm{eV}$ in this excited state. This problem was solved by van der Lugt and Oosterhoff $[152,171]$ who found that the dark state 2A can have a low-lying minimum, the pericyclic minimum ( $\left.\mathrm{p}^{*}\right)$ resulting from an avoided crossing of the potentials correlating the reactant ground state and a two-electron excited product state and the corresponding pair for the backward reaction. (Two recent calculations with time-dependent density-functional theory for technical reasons considered the 1B state alone and found a CI with $\mathrm{S}_{0}$ on the hexatriene side $[172,173]$. But the present and previous [34, $151,154]$ time-resolved spectroscopy shows clear evidence that the path passes via the $2 \mathrm{~A}$ state.) However, the calculations usually show two $2 \mathrm{~A}$ minima, a lower one in the WHallowed and a higher one in the WH-forbidden direction, both easily accessible (see, e.g. [152] for cyclobutene or Fig. 3 of [174] for butadiene); continuing in anti-WH direction, the exit of the latter has a barrier. According to our results, the molecules in the "wrong" minimum cannot simply go back and then find the allowed minimum in the second attempt; this path would imply an activation energy, and therefore this process would be too slow to be compatible with our observed short times.

The problem is solved by considering more than one reaction, taking also into account that the initial acceleration is towards the FC-active coordinates, with the $\mathrm{WH}$ rules being turned on only later. In CHD, most molecules (those excited from the lower-energy chair-like conformer with $C_{2}$ symmetry) initially follow a conrotatory path; thereafter, the WH-type interactions facilitate its continuation via the first $\mathrm{CI}$ to the $2 \mathrm{~A}$ minimum and the last $\mathrm{CI}$ towards the allowed ring opening. But a small fraction of the molecules (those excited from higher-energy boat-like conformer with $C_{\mathrm{s}}$ symmetry) is accelerated towards a disrotatory direction [175]. But then (probably only in the 2A state, if the calculations for butadiene [174] can be generalized) a barrier resulting from WH interactions hinders continuation towards disrotatory ring opening. Instead, from there the molecules (such as many dienes) can follow a barrierless WH-allowed path to disrotatory ring closure to a cyclobutene derivative (bicyclo[2,2,0]hexene-2). (That the diene $\rightarrow$ cyclobutene path is barrierless and ultrafast was found 
for cycloheptadiene and cyclooctadiene in [59].) It was pointed out in [175] that CHD derivatives favouring the $C_{\mathrm{s}}$-symmetric conformer in fact prefer this disrotatory path. Hence in these (and probably more) examples, the acceleration direction in the FC region controls, which of several WH-allowed reactions is initiated later on.

On the basis of the calculated lengthening of the $\mathrm{CH}_{2}-\mathrm{CH}_{2}$ distance (Fig. 5.4.1.1), it was suggested that the ring opening is already more or less complete before leaving the $1 \mathrm{~B}$ surface $[176,177]$. The ring vibrations observed here, however, demonstrate that the ring is still intact, though weakened, even in the dark state. In principle, in the latter case most of the restoring force could be located in the rest of the backbone, not in the weak three-electron three center bond (Scheme 1). But the previous investigation from this laboratory shows that the geometrical structure of hexatriene is only reached on arrival on the $\mathrm{S}_{0}$ surface, some distance after the last CI (location $\mathrm{L}_{5}$ in [34]). This is probably characteristic of all nonadiabatic photochemical processes: The reaction already begins on the excited surface(s) but is only half completed near the location of the radiationless transition (through a CI) to the ground-state surface.

The observed coherent oscillations also confirm the previous (e.g. [151]) ideas on the reaction path: Initial motion along $\mathrm{FC}$-active coordinates ( $\mathrm{CC}$ stretch and conrotatory $\mathrm{CC}$ torsion), which after some excursion still on the 1B surface activates WH interactions (because the $\mathrm{CH}_{2}-\mathrm{CH}_{2}$ bond is now more parallel to the $\pi$ orbitals); then on surrounding the $1 \mathrm{~B} / 2 \mathrm{~A} \mathrm{CI}$, acceleration to a symmetry-breaking direction that leads directly to a part (probably not the minimum) of the $2 \mathrm{~A} / 1 \mathrm{~A}$ IS. The wave packet moves in "record time", within a small fraction of the periods of the torsional vibrations along the local reaction coordinates; this is ascribed to early acceleration in regions of the potential, where it is still steeper. The largest part of wave packet is transmitted through the two CIs at the first attempt; only a smaller part passes by, oscillates and returns to the CI. This part is small enough and so on arrival at the groundstate surface, the wave packet is still compact.

In view of this more or less ballistic motion, one may ask whether the analysis by rate equations (modified by periodic modulation functions) is justified. If the observation windows, associated with the probing technique, were very narrow, the signal would reflect the shape of the wave packet passing by the window; this would not be exponential. However, these windows are broad (symbolized by the lengths of the arrows in the figures), and the ionization probability continuously varies within them. This may contribute to wash out in the signals 
some signatures of a coherent or ballistic motion. A closer theoretical analysis would be desirable, however. It is a related idea that we write the time constants next to the arrows, not to localized points ("states"): they mostly reflect the travelling times of the wave packet, not any time for transmission through a bottleneck.

To summarize the reaction path following the initial excitation: the molecule is first in the FC region $\left(\mathrm{L}_{1}\right)$ accelerated towards $\mathrm{CC}$ stretching in the $\pi$ system and a symmetric $\mathrm{C}=\mathrm{C}$ twist. The latter motion - after some excursion - also distorts and stretches the $\mathrm{CH}_{2}-\mathrm{CH}_{2}$ bond, so that $\mathrm{WH}$ interactions are activated after this delay (in $\mathrm{L}_{2}$ ). On leaving $\mathrm{L}_{2}$ (the $1 \mathrm{~B}$ minimum) around the lower cone of the $1 \mathrm{~B} / 2 \mathrm{~A} \mathrm{CI}$, the wave packet is rapidly accelerated along an antisymmetric coordinate, which breaks the $C_{2}$ symmetry of the molecule and eventually leads to a ballistic path to (and through) the last (2A/1A) CI. The ring opening begins already on the 1B surface, is far advanced near the 2A minimum, but is only completed on the ground-state surface. 


\section{6}

\section{The dynamics of the dissociation of $\operatorname{Cr}(\mathrm{CO})_{6}$}

\subsection{Experimental}

The molecular system and the reaction investigated here is the chromium hexacarbonyl, $\mathrm{Cr}(\mathrm{CO})_{6}$, excited in the wavelength range $270-345 \mathrm{~nm}$ and the subsequent elimination of one carbonyl ligand (CO group). In this molecule, one can excite in the near UV quite a number of electronic states. The nature and multiplicity (singlet or triplet) of some of them is controversial. The multiplicity has important consequences for the chemical properties of the catalytically active - dissociation product. It is hence desirable to have a tunable source of short UV pulses to investigate the dynamics. This was indeed one of the motivations to develop such a laser source.

The laser system and the experimental set-up used to generate the ultrashort 810 -nm probe $\left(10^{13} \mathrm{~W} \mathrm{~cm}^{-2}, 810 \mathrm{~nm}, \sim 10-20 \mathrm{fs}\right)$ and the UV pump pulses for the time-resolved studies were described in Chapter 2; the pump wavelengths used lie in the region of the third harmonic at $270 \mathrm{~nm}$ (30 fs) or at 318 (typically $10^{9} \mathrm{~W} \mathrm{~cm}^{-2}, \sim 30 \mathrm{fs}$ ) and $345 \mathrm{~nm}$. The 318-nm pulses are taken from the supercontinuum radiation after reflection from dielectric mirrors at this wavelength region (Chapter 2). The weak absorption $\left(2 \times 10^{-18} \mathrm{~cm}^{2}\right)$ of $\mathrm{Cr}(\mathrm{CO})_{6}$ at $345 \mathrm{~nm}$ requires a stronger pump pulse and therefore the radiation generated in the commercial (TOPAS, Light Conversion) optical parametric generator combined with various nonlinear frequency conversion steps is used, as in e.g. [59]. It delivers pulses of a few $\mu \mathrm{J}$ with durations of $42 \mathrm{fs}$ after a prism compressor (Chapter 2).

As in all previous cases $\mathrm{Xe}$ is used to determine the time zero and the pulse duration of the UV pump pulses. The pressure of $\mathrm{Cr}(\mathrm{CO})_{6}$ in the ionization region of the mass-spectrometer 
is $10^{-4}$ to $10^{-7}$ mbar. Time-resolved data are presented on the parent $\mathrm{Cr}(\mathrm{CO})_{6}{ }^{+}$and fragment $\mathrm{Cr}(\mathrm{CO})_{m}{ }^{+}, m=5 . .3$, ions.

\subsection{Background}

$\mathrm{Cr}(\mathrm{CO})_{6}$ is an octahedral complex and belongs to the $O_{\mathrm{h}}$ symmetry point group (Fig. 6.2.1).

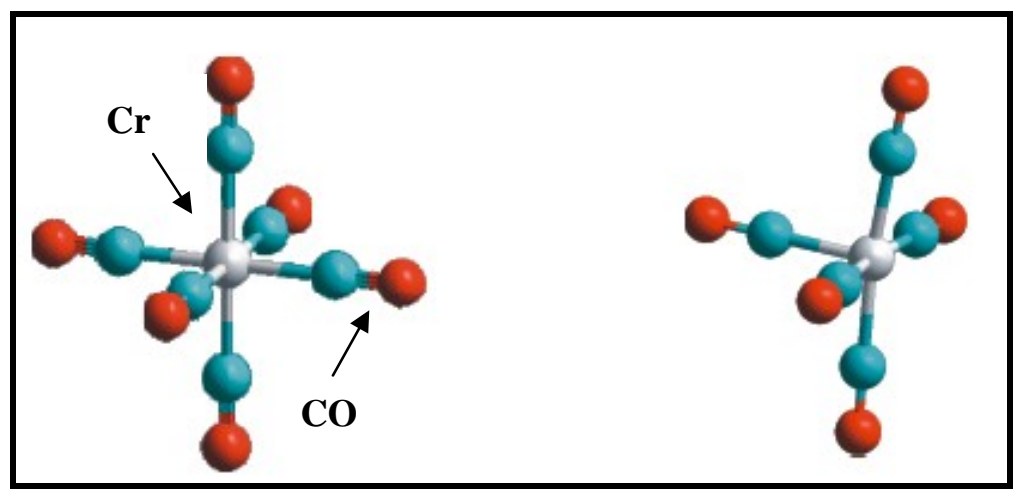

Fig. 6.2.1 $\mathrm{Cr}(\mathrm{CO})_{6}$ and $\mathrm{Cr}(\mathrm{CO})_{5}$.

The electronic structure of $\operatorname{Cr}(\mathrm{CO})_{6}$ is mainly determined by the contribution of the $3 d, 4 s$ and $4 p$ atomic orbitals of the metal and of linear combinations (with the appropriate symmetry) of the 6 HOMOs and the 12 LUMOs of the CO ligands; the result is molecular orbitals of octahedral symmetry, delocalized over the whole complex [178-180]. The HOMO of CO is a nonbonding orbital of $\sigma$ character (i.e. of axial symmetry along the $\mathrm{CO}$ bond), occupied by a pair of electrons mainly belonging to $\mathrm{C}$, whereas the LUMO of CO is a $\pi^{*}$ antibonding orbital $[181,182]$. In the presence of the ligands the $d$ manifold of $\mathrm{Cr}$ orbitals is subject to ligandfield splitting, giving rise to three degenerate $t_{2 \mathrm{~g}}$ orbitals $\left(d_{\mathrm{xy}}, d_{\mathrm{xz}}, d_{\mathrm{yz}}\right)$ and two degenerate $e_{\mathrm{g}}$ orbitals. The ground state of $\mathrm{Cr}(\mathrm{CO})_{6},{ }^{1} \mathrm{~A}_{1 \mathrm{~g}}$, has the electron configuration (core) $\left(5 e_{\mathrm{g}}\right)^{4}$ $\left(2 t_{2 \mathrm{~g}}\right)^{6}$. The doubly degenerate $e_{\mathrm{g}}$ orbitals are a linear combination of the $\mathrm{Cr}$ empty $e_{\mathrm{g}}$ and the fully occupied $\sigma \mathrm{CO}$ orbitals, so that a $\sigma$ bond with ligand-to-metal donation is formed, while the triply degenerate $t_{2 \mathrm{~g}}$ orbitals are formed by the fully occupied $t_{2 \mathrm{~g}}$ orbital of the metal and the empty $\pi^{*} \mathrm{CO}$ orbitals, so that a $\pi$ bond between the metal and the ligand is formed ( $\pi$ back bonding).

Two important classes of transitions constitute the so called metal-to-ligand charge-transfer (MLCT) and the ligand-field (LF) transitions. The former involve the promotion of one electron from the Cr-centred $d$ orbital ( $t_{2 \mathrm{~g}}, \mathrm{HOMO}$ ) to a nonbonding (with respect to the $\mathrm{Cr}-\mathrm{CO}$ bond), ligand-localized, $\pi^{*}$ orbital of the carbonyl; the latter arise from the transition from the 
same $t_{2 \mathrm{~g}}$ orbital to an $e_{\mathrm{g}}^{*}$ orbital, which is $\sigma$-antibonding with respect to the $\mathrm{Cr}-\mathrm{CO}$ bond and is largely metal-centered too [179, 183-185].

The absorption spectrum of $\mathrm{Cr}(\mathrm{CO})_{6}$ in the UV region (Fig. 6.2.2) and its assignment have been extensively studied since the first gas-phase spectrum of [178], most recently by Hummel et al. [186] and Ben Amor et al. [187]. It is now established [179, 184, 185] that the main two absorption bands of the spectrum reflect the ${ }^{1} \mathrm{~A}_{1 \mathrm{~g}} \rightarrow 1^{1} \mathrm{~T}_{1 \mathrm{u}}\left(2 \mathrm{t}_{2 \mathrm{~g}} \rightarrow 9 t_{1 \mathrm{u}}\right)$ and ${ }^{1} \mathrm{~A}_{1 \mathrm{~g}} \rightarrow 2^{1} \mathrm{~T}_{1 \mathrm{u}}$ $\left(2 t_{2 \mathrm{~g}} \rightarrow 2 t_{2 \mathrm{u}}\right)$ MLCT transitions, which are the only symmetry and spin allowed transitions. They lie around 280 and $220 \mathrm{~nm}$ respectively. The calculations of $[179,184,185]$ predict many symmetry forbidden MLCT transitions between and beyond these two bands, as well as the LF transitions ${ }^{1} \mathrm{~A}_{1 \mathrm{~g}} \rightarrow{ }^{1} \mathrm{~T}_{1 \mathrm{~g}}$ and ${ }^{1} \mathrm{~A}_{1 \mathrm{~g}} \rightarrow{ }^{1} \mathrm{~T}_{2 \mathrm{~g}}$. The LF potentials are repulsive with respect to the $\mathrm{Cr}-\mathrm{CO}$ bond and therefore lead to dissociation. In the long-wavelength region, below $270 \mathrm{~nm}$, lie some more symmetry-forbidden MLCT states. The absorption spectrum is shown in Fig. 6.2. 2 .

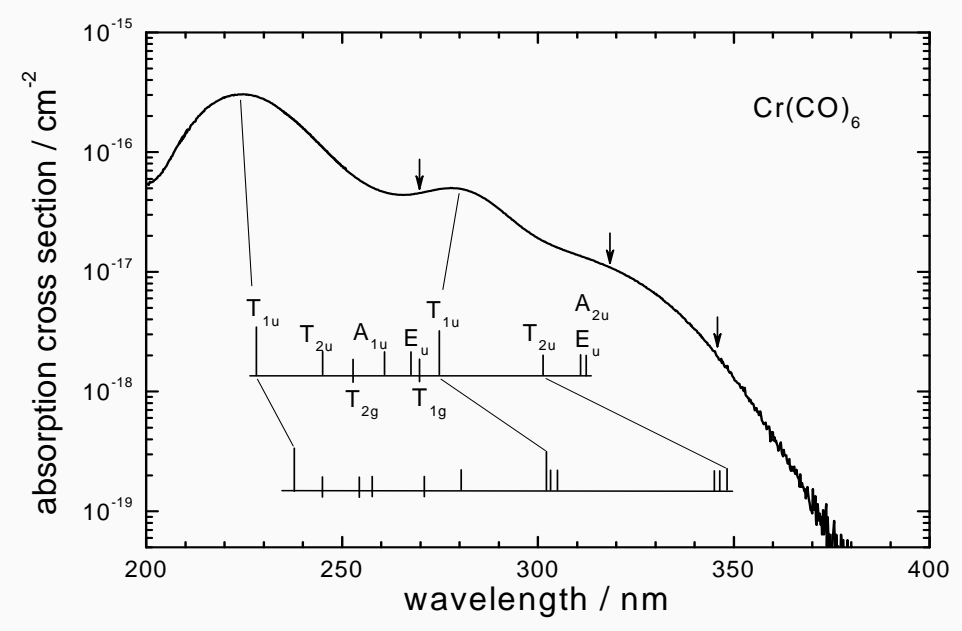

Fig. 6.2.2 Gas-phase absorption spectrum of $\mathrm{Cr}(\mathrm{CO})_{6}$ taken from [8]. The two symmetry- and spin-allowed MLCT transitions have their maxima near $280 \mathrm{~nm}$ and $220 \mathrm{~nm}$. The LF transitions are the symmetry-forbidden ${ }^{1} \mathrm{~A}_{1 \mathrm{~g}} \rightarrow{ }^{1} \mathrm{~T}_{2 \mathrm{~g}}$ and ${ }^{1} \mathrm{~A}_{1 \mathrm{~g}} \rightarrow{ }^{1} \mathrm{~T}_{1 \mathrm{~g}}$ transitions. The symmetry-forbidden MLCT transitions lie between the main two absorption bands at 220 and $280 \mathrm{~nm}$, as well as beyond $280 \mathrm{~nm}$. The calculated positions are from [184] and [187]. The arrows indicate the pump wavelengths.

The photo-dissociation of $\mathrm{Cr}(\mathrm{CO})_{6}$ has been investigated in the past both theoretically, e.g. $[179,185,188]$ and by time-resolved spectroscopy, e.g. [189]. Experiments have shown that upon excitation of $\mathrm{Cr}(\mathrm{CO})_{6}$ to an MLCT state at $270 \mathrm{~nm}$ the molecule eliminates one CO li- 
gand and $\mathrm{Cr}(\mathrm{CO})_{5}$ is formed (Fig. 6.2.1). It is generally accepted that from this initially excited MLCT state the molecule first undergoes an internal conversion to a LF state which subsequently leads to the dissociation. This is confirmed experimentally by Trushin et al. who have studied the dynamics of the dissociation of the metal carbonyl complexes $\mathrm{M}(\mathrm{CO})_{6}, \mathrm{M}=$ Cr, Mo, W $[189,190]$ and is extended to all initial excitations in the region $220-345 \mathrm{~nm}$ [8]. Moreover, theory $[179,180,185]$ suggests one relaxation step in the intact $\operatorname{Cr}(\mathrm{CO})_{6}$ from the lowest MLCT $\left(\mathrm{T}_{2 \mathrm{u}}\right)$ state to a dissociative LF state; it was not specified how the molecule arrives to $\mathrm{T}_{2 \mathrm{u}}$ from an initially excited state such as $\mathrm{T}_{1 \mathrm{u}}$.

Before high-level calculations [179, 184, 185] were feasible with complexes such as $\mathrm{Cr}(\mathrm{CO})_{6}$, it was assumed [178] (and considered established in textbooks, e.g. [191] and [182]) that the weak longer-wavelength transitions lead to triplet states (instead of the symmetryforbidden singlet MLCT states); also a recent high-level calculation [187] supports this view again. Triplet products would radically differ in their chemical reactions from the corresponding singlet molecules. Note that the dissociation product $\mathrm{Cr}(\mathrm{CO})_{5}$ is a prototype of catalytically active species in organometallic chemistry. By comparing the dynamics at the different wavelengths we show that only singlet states are involved.

\subsection{Results and analysis}

\subsubsection{Dynamics of $\mathrm{Cr}(\mathrm{CO})_{6}$ excited at $270 \mathrm{~nm}$}

This wavelength populates the $1^{1} \mathrm{~T}_{1 \mathrm{u}}$ MLCT state (Fig. 6.2.2). Fig. 6.3.1.1 - 2 show the measured ion yields for $\mathrm{Cr}(\mathrm{CO})_{m}{ }^{+}$with $m=6 \ldots 3$ together with simulation curves, and Table 6.3.1.1 lists the time constants $\tau_{i}$ and ionization cross sections ${ }^{m} \sigma_{i}$ evaluated from these data. The smaller fragments were not evaluated in much detail, since the time constants and oscillation frequencies agreed with those of the other signals and/or those of [189]. It is practical to first extract from them the later time constants, outside the pump-probe overlapping region, where convolution with the instrumental function is not necessary. They agree with the values found in $[189,190]\left(\tau_{4}=930\right.$ fs and $\tau_{5}=\infty$, i.e. $>500 \mathrm{ps}$, both from $\mathrm{Cr}(\mathrm{CO})_{m}{ }^{+}$with $m=3 \ldots 0$, not shown in this long-time range, although a trace of the $\tau_{4}$ process can be seen in the inset of Fig. 6.3.1.2c for $\left.\mathrm{Cr}(\mathrm{CO})_{4}{ }^{+}\right)$. These time constants are then fixed in the next step, in which a full fit beginning from the earliest times, with convolution is performed (according to the temporal function derived in Chapter 3). 
A single exponential fit to $\mathrm{Cr}(\mathrm{CO})_{5}{ }^{+}$and $\mathrm{Cr}(\mathrm{CO})_{4}{ }^{+}$in the range of $120-230$ fs (Fig. 6.3.1.2a, c) reveals a time constant $\tau_{3}=40 \mathrm{fs}\left(\mathrm{L}_{4}\right.$ does not contribute to the $\mathrm{Cr}(\mathrm{CO})_{5}{ }^{+}$signal and nearly not to $\mathrm{Cr}(\mathrm{CO})_{4}{ }^{+}$Table 6.3 .1 .1$)$, later confirmed by a fit over the full time range, including the other time constants and the oscillations. Note that $\mathrm{Cr}(\mathrm{CO})_{4}{ }^{+}$signal shows a strong oscillatory modulation at later times (discussed below). With $\tau_{3}$ fixed, $\tau_{2}=18$ fs is then determined from $\mathrm{Cr}(\mathrm{CO})_{5}{ }^{+}$by a double exponential fit for times $\geq 60 \mathrm{fs}$ (Fig. 6.3.1.2a). Finally, the preceding $\tau_{1}=12.5$ fs could be determined by a single exponential fit (with convolution in the full fit or without in the range 40-110 fs outside the pump-probe overlapping region) from the parent $\mathrm{Cr}(\mathrm{CO})_{6}^{+}$, which is only produced from $\mathrm{L}_{1}$ and from nowhere else (Fig. 6.3.1.1, Table 6.3.1.1). This value for $\mathrm{L}_{1}$ was also confirmed with better time resolution (not shown here, see [4]), where the pump pulses at $270 \mathrm{~nm}$ had a duration of $10 \mathrm{fs}$.

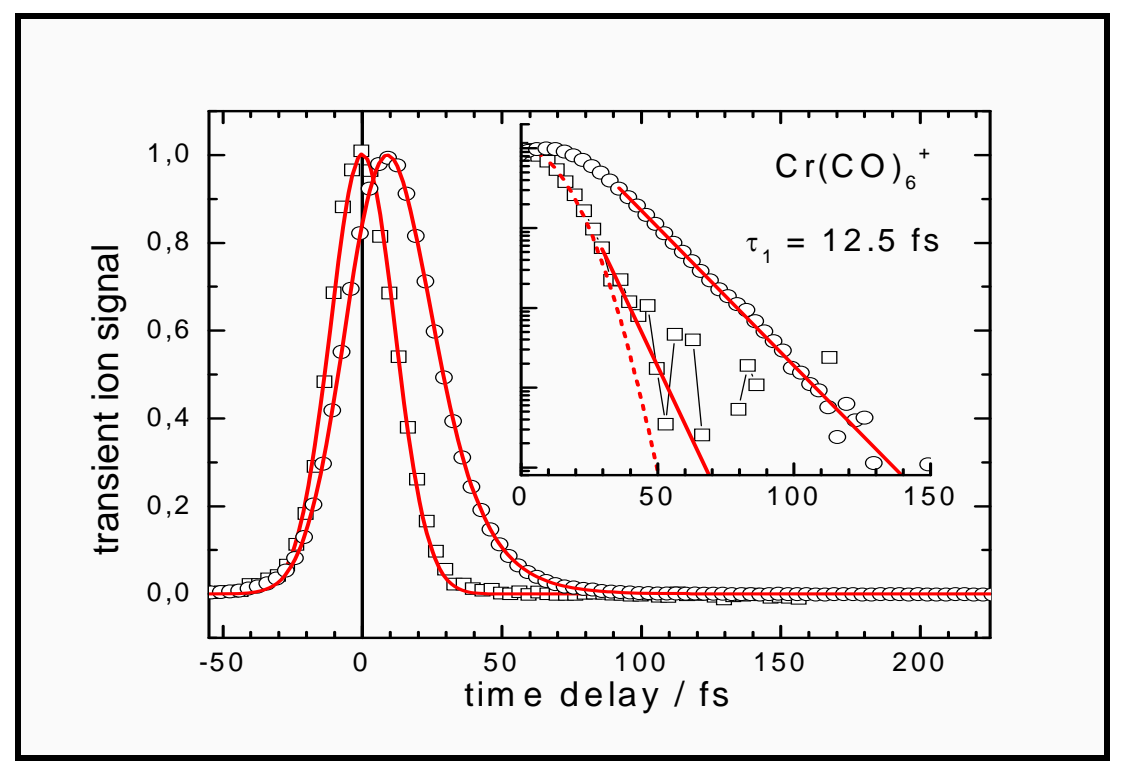

Fig. 6.3.1.1 Measured ion yield of $\mathrm{Cr}(\mathrm{CO})_{6}{ }^{+}$(circle symbols) using the pump pulses at $270 \mathrm{~nm}$ of duration 30 fs together with the simulation curves (single exponential fit (inset) or full fit). The $\mathrm{Xe}^{+}$data represent the cross correlation of the pump with the fifth power of the probe, indicating the instrumental function (square symbols).

At this step, the above set of time constants is used to check whether all signals can be reproduced by them over the full time range, either to improve their values or to detect from deviations any further process. An obvious deviation is the oscillation in the decaying part $\left(\tau_{4}=40 \mathrm{fs}\right)$ of the $\mathrm{Cr}(\mathrm{CO})_{4}^{+}$signal and a closer inspection reveals more such modulations. 

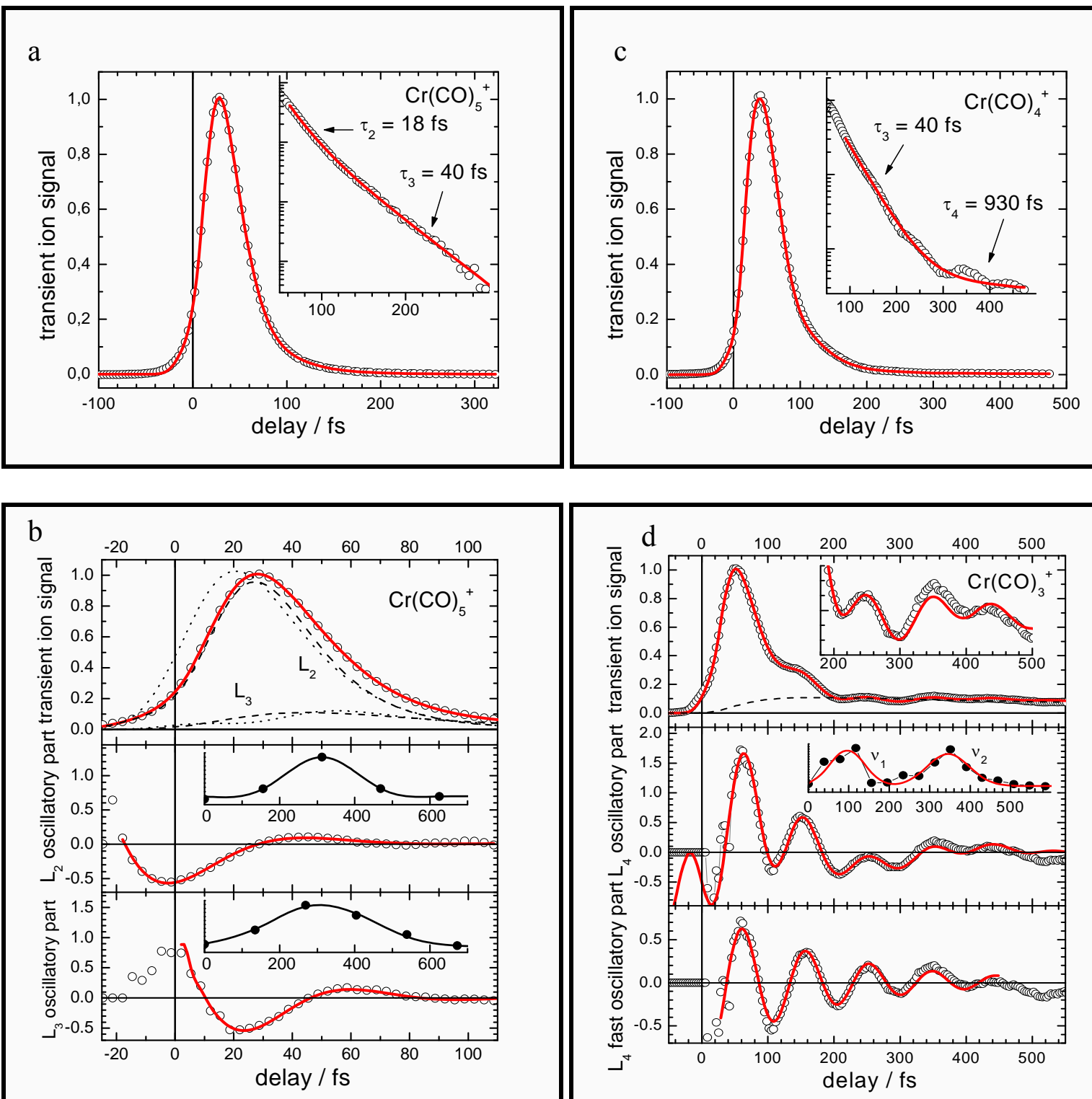

Fig. 6.3.1.2 Ion yield of (a) $\mathrm{Cr}(\mathrm{CO})_{5}^{+}$with 270-nm 30-fs pump, together with simulation curves, (b) the $\mathrm{Cr}(\mathrm{CO})_{5}{ }^{+}$signal is simulated by contributions (thin lines) from $\mathrm{L}_{2}$ and $\mathrm{L}_{3}$; the dotted thin lines only contain the exponential parts, the broken lines also the oscillations (function $f_{\text {osc }}$, see below). The latter are isolated in the last two panels, with the insets showing their Fourier transforms (abscissa unit: $\left.\mathrm{cm}^{-1}\right),(\mathrm{c}) \mathrm{Cr}(\mathrm{CO})_{4}{ }^{+}$with full fit and double exponential fit (inset), (d) $\mathrm{Cr}(\mathrm{CO})_{3}{ }^{+}$with full fit (the later part of the signal is magnified in the inset) and the oscillatory part (second panel) of window $\mathrm{L}_{4}$; the inset shows the two frequencies arising from the Fourier transform. The faster oscillation $\left(360 \mathrm{~cm}^{-1}\right)$ is shown in the last panel.

\begin{tabular}{|c|c|c|c|c|c|}
\hline $\mathrm{L}_{i}$ & $\mathrm{~L}_{1}$ & $\mathrm{~L}_{2}$ & $\mathrm{~L}_{3}$ & $\mathrm{~L}_{4}$ & $\mathrm{~L}_{5}$ \\
\hline$\tau_{i} / \mathrm{fs}$ & $12.5 \pm 0.5$ & $18 \pm 2$ & $40 \pm 3$ & $930 \pm 100$ & $\infty$ \\
\hline $\mathrm{Cr}(\mathrm{CO})_{6}{ }^{+}$ & 1 & 0 & 0 & 0 & 0 \\
\hline $\mathrm{Cr}(\mathrm{CO})_{5}{ }^{+}$ & 0 & 1 & 0.082 & 0 & 0 \\
\hline $\mathrm{Cr}(\mathrm{CO})_{4}{ }^{+}$ & 0 & 1 & 0.440 & 0.002 & 0 \\
\hline $\mathrm{Cr}(\mathrm{CO})_{3}{ }^{+}$ & 0 & 0 & 1 & 0.063 & 0.013 \\
\hline
\end{tabular}


Table 6.3.1.1 Pump laser at $270 \mathrm{~nm}$ : life times $\tau_{i}$ and effective ionization-dissociation cross sections ${ }^{m} \sigma_{i}$. In the locations $\mathrm{L}_{2}-\mathrm{L}_{4}$, periodic modulations of ${ }^{m} \sigma_{i}$ were also used. The data for $\mathrm{Cr}(\mathrm{CO})_{m}{ }^{+}$with $m=2 . .0$ were not evaluated in detail. Error limits are standard deviations for the values determined in $\geq 8$ different scans.

To extract the parameters of a modulation, the easiest is to begin with a signal in a time range, where practically only one location contributes. For $\mathrm{Cr}(\mathrm{CO})_{4}^{+}$, in the range $120-230$ fs, $L_{2}$ has already decayed $\left(\tau_{2}=18 \mathrm{fs}\right)$, while $L_{4}$ contributes very little to the signal $\left({ }^{4} \sigma_{4}\right.$ is small, 0.002 , Table 6.3.1.1). This part of the signal is divided by the decaying $\mathrm{L}_{3}$ population (the result being $\left.\propto\left(1+f_{\text {osc }}\right)\right)$ and is then Fourier transformed to obtain the period $T_{\text {osc }}(73 \mathrm{fs}$, corresponding to a wavenumber of $465 \mathrm{~cm}^{-1}$ ). This value is then inserted in $f_{\text {osc }}=A \exp \left(-t / \tau_{\text {deph }}\right) \cos (2 \pi \mathrm{c} v t-\varphi)$, which is remultiplied by the time-dependent $\mathrm{L}_{3}$ population and used to simulate this part of the $\mathrm{Cr}(\mathrm{CO})_{4}^{+}$signal; $\tau_{\text {deph }}$ and $\varphi$ (Table 6.3.2.2) are obtained in this way and the quality of the fit is judged. Since $\mathrm{L}_{3}$ contributes to $\mathrm{Cr}(\mathrm{CO})_{5}{ }^{+}$too, the same modulation (with the same $v$ and $\tau_{\text {deph }}$, but possibly different $A$ and $\varphi$ ) is expected in this signal too, which is therefore simulated by the exponential $\mathrm{L}_{2}$ and $\mathrm{L}_{3}$ populations (with the $\tau_{i}$ and ${ }^{4} \sigma_{i}$ determined above) each multiplied by the periodic functions $\left(1+f_{\text {osc }}\right)$, with $v$ for $\mathrm{L}_{3}$ now fixed. (The modulated $\mathrm{L}_{3}$ contribution is shown in the lowest panel of Fig. 6.3.1.2b.) This results also in a value for $v\left(362 \mathrm{~cm}^{-1}, 93 \mathrm{fs}\right.$ ) for $\mathrm{L}_{2}$ (middle panel of Fig. 6.3.1.2b). Because of the short $\tau_{1}(12.5 \mathrm{fs})$, one cannot expect to detect such an oscillation also in $\mathrm{L}_{1}$.

The more prominent oscillations observed from $\mathrm{L}_{4}$ are illustrated in Fig. 6.3.1.2d with the $\mathrm{Cr}(\mathrm{CO})_{3}{ }^{+}$signal. Subtracting the $\mathrm{L}_{3}$ contribution from the total signal (upper panel) and dividing by the $\mathrm{L}_{4}$ population (broken line) results in the periodic modulation ( $f_{\text {osc }}$ shown in the middle panel, with the Fourier transform as inset); after subtracting the slowly oscillating part ( $350 \mathrm{fs}, 95 \mathrm{~cm}^{-1}$ ), the fast oscillation $\left(95 \mathrm{fs}, 350 \mathrm{~cm}^{-1}\right.$ ) is clearly revealed in the lower panel. The oscillation data are summarized below. 


\subsubsection{Dynamics of $\mathrm{Cr}(\mathrm{CO})_{6}$ excited at 318 and $345 \mathrm{~nm}$}

Whereas $270 \mathrm{~nm}$ is near the maximum of the $\mathrm{T}_{1 \mathrm{u}}$ absorption, the wavelengths at 318 and 345 nm coincide with two weaker bands, which according to Beach and Gray [178] can be discerned in the long-wavelength wing of the gas-phase spectrum (Fig. 6.2.2).

The time-resolved measurements and evaluations for the yield of $\mathrm{Cr}(\mathrm{CO})_{m}{ }^{+}$with $m=6 . .4$ at both wavelengths were done in the same way as at $270 \mathrm{~nm}$ (see also [8]). The time constants are summarized in Table 6.3.2.1 and the data for the oscillations in Table 6.3.2.2 (where the values for the 270-nm excitation are also shown). At all wavelengths, the signals are simulated by the same number of time constants, their values varying by less than a factor of 2 . Although the number of oscillations also remains the same, with the longer wavelengths an oscillation is found in the parent ion too and hence in $\mathrm{L}_{1}$, in contrast to the $270-\mathrm{nm}$ case. It might well be that this is due to the nearly twice longer lifetimes $\tau_{1}$ at 318 and $345 \mathrm{~nm}$ that provide a chance to detect a periodic modulation (note that $T_{\mathrm{osc}}>\tau_{1}$ ). (This idea is further supported by the fact that such an oscillation $\left(T_{\text {osc }}=108 \mathrm{fs}, \tau_{\text {deph }}=34 \mathrm{fs}\right)$ is detected in the parent ion of $\mathrm{Mo}(\mathrm{CO})_{6}$ as well [8]; investigation of the initial dynamics of the dissociation of $\operatorname{Mo}(\mathrm{CO})_{6}[8]$ revealed two time constants $\tau_{1}=26 \mathrm{fs}$ and $\tau_{2}=35 \mathrm{fs}$, after excitation at $270 \mathrm{~nm}$, i.e. in the wing of the $T_{1 u}$ band at $286 \mathrm{~nm}$. Again, the longer $\tau_{1}$ may have facilitated the observation of an oscillation.)

In a first variant of the evaluation, $v$ in $\mathrm{L}_{2}-\mathrm{L}_{4}$ is fixed to the values found with $270 \mathrm{~nm}$, given the fact that these locations on the PES represent electronic states of $\mathrm{Cr}(\mathrm{CO})_{5}$ reached in all cases. However, vibrational frequencies can (slightly) depend on the available excess energy, for example due to anharmonicity. Indeed, when $T_{\text {osc }}$ in $\mathrm{L}_{2}-\mathrm{L}_{4}$ is allowed to vary in the fit procedure the quality of the simulation improves a little with oscillation frequencies that are lower by about $10 \%$ at 318 than at $270 \mathrm{~nm}$. Because this variation is near the error limit of the fixed values, Table 6.3.2.2 lists only the latter.

\begin{tabular}{|c|c|c|c|c|}
\hline$\lambda_{\text {pump }}$ & $\tau_{1} / \mathrm{fs}$ & $\tau_{2} / \mathrm{fs}$ & $\tau_{3} / \mathrm{fs}$ & $\tau_{4} / \mathrm{fs}$ \\
\hline $270 \mathrm{~nm}$ & $12.5 \pm 0.5$ & $18 \pm 2$ & $40 \pm 3$ & $930 \pm 100$ \\
\hline $318 \mathrm{~nm}$ & $19 \pm 1$ & $20 \pm 2$ & $35 \pm 3$ & $1200 \pm 100$ \\
\hline $345 \mathrm{~nm}$ & $21 \pm 1$ & $22 \pm 2$ & $25 \pm 3$ & $1500 \pm 100$ \\
\hline
\end{tabular}


Table 6.3.2.1 Time constants $\tau_{i}$ found for $\mathrm{Cr}(\mathrm{CO})_{6}$ with different pump wavelengths $\lambda_{\text {pump }}$. Error limits are standard deviations for the values determined in $\geq 8$ different scans.

\begin{tabular}{|c|c|c|c|c|c|c|c|c|c|c|c|c|}
\hline \multirow[b]{2}{*}{$\lambda_{\text {pump }} / \mathrm{nm}$} & \multicolumn{3}{|c|}{$\mathrm{L}_{1}$} & \multicolumn{3}{|c|}{$\mathrm{L}_{2}$} & \multicolumn{3}{|c|}{$\mathrm{L}_{3}$} & \multicolumn{3}{|c|}{$\mathrm{L}_{4}$} \\
\hline & $T_{\mathrm{osc}} / \mathrm{fs}$ & $\tau_{\text {deph }} / \mathrm{fs}$ & $\varphi / 2 \pi$ & $T_{\text {osc }} / \mathrm{fs}$ & $\tau_{\mathrm{deph}} / \mathrm{fs}$ & $\varphi / 2 \pi$ & $T_{\text {osc }} / \mathrm{fs}$ & $\tau_{\text {deph }} /$ fs & $\varphi / 2 \pi$ & $T_{\text {osd }} / f s$ & $\tau_{\text {deph }} /$ fs & $\varphi / 2 \pi$ \\
\hline 270 & - & - & - & $93 \pm 10$ & $27 \pm 10$ & $0.58 \pm 0.05$ & $73 \pm 10$ & $37 \pm 10$ & $0.88 \pm 0.05$ & $95 \pm 3$ & $165 \pm 50$ & $0.67 \pm 0.05$ \\
\hline 318 & $86.5 \pm 15$ & $150 \pm 100$ & $0.36 \pm 0.10$ & $93 \pm 10$ & $76 \pm 20$ & $0.52 \pm 0.05$ & $73 \pm 10$ & $40 \pm 10$ & $0.9 \pm 0.05$ & $95 \pm 5$ & $200 \pm 50$ & $0.6 \pm 0.05$ \\
\hline 345 & $86.5 \pm 15$ & $200 \pm 100$ & $0.36 \pm 0.10$ & $93 \pm 10$ & $70 \pm 30$ & $0.66 \pm 0.05$ & $73 \pm 10$ & $40 \pm 10$ & $0.16 \pm 0.05$ & $95 \pm 7$ & $270 \pm 50$ & $0.58 \pm 0.05$ \\
\hline $\begin{array}{l}v_{i} / \mathrm{cm}^{-1} \text { at } \\
270-345\end{array}$ & $397 \pm 69$ & & & $362 \pm 39$ & & & $465 \pm 64$ & & & $352 \pm 26$ & & \\
\hline
\end{tabular}

Table 6.3.2.2 Fast oscillations (period $T_{\text {osc }}$ corresponding to wavenumber $v$, dephasing time $\tau_{\text {deph }}$, phase delay $\varphi$ ) observed in the different observation windows $\mathrm{L}_{i}$. Error limits indicate the range, in which reasonably good fits are possible. The slow oscillation ( $350 \mathrm{fs}, 95 \mathrm{~cm}^{-1}$ ), observed only in $\mathrm{L}_{4}$, is the same as the one reported with $267 \mathrm{~nm}$ pump in [189].

Finally, Table 6.3.2.3 reports on the initial dynamics $\left(\tau_{1}\right)$ of $\mathrm{Cr}(\mathrm{CO})_{6}$ excited at 290, 242, 224, 196 and $162 \mathrm{~nm}$ for comparison (see [8]).

\begin{tabular}{|c|ccccc|}
\hline & $\mathrm{Cr}(\mathrm{CO})_{6}$ & $\mathrm{Cr}(\mathrm{CO})_{6}$ & $\mathrm{Cr}(\mathrm{CO})_{6}$ & $\mathrm{Cr}(\mathrm{CO})_{6}$ & $\mathrm{Cr}(\mathrm{CO})_{6}$ \\
$\lambda_{\text {pump }} / \mathrm{nm}$ & $282[8]$ & $242[59]$ & $224[59]$ & $196[8]$ & $162[8]$ \\
\hline$\tau_{1} / \mathrm{fs}$ & $14 \pm 1$ & $16 \pm 2$ & $20 \pm 2$ & $55 \pm 5$ & $406 \pm 20$ \\
\hline
\end{tabular}

Tab. 6.3.2.3 First time constants $\tau_{1}$ found in $\mathrm{Cr}(\mathrm{CO})_{6}$ (from the $\mathrm{Cr}(\mathrm{CO})_{6}{ }^{+}$signal) with different pump wavelengths.

\subsection{Discussion}

\subsubsection{Assignment of the lifetimes and oscillations}

A correlation scheme of the potentials of $\mathrm{Cr}(\mathrm{CO})_{6}$ and $\mathrm{Cr}(\mathrm{CO})_{5}$ is suggested in Fig. 6.4.1.1. With $267 \mathrm{~nm}$, the assignment of the observed lifetimes and oscillations is discussed in detail in [189] and the steps of the dissociation were confirmed by high-level calculations [188, 192]. 


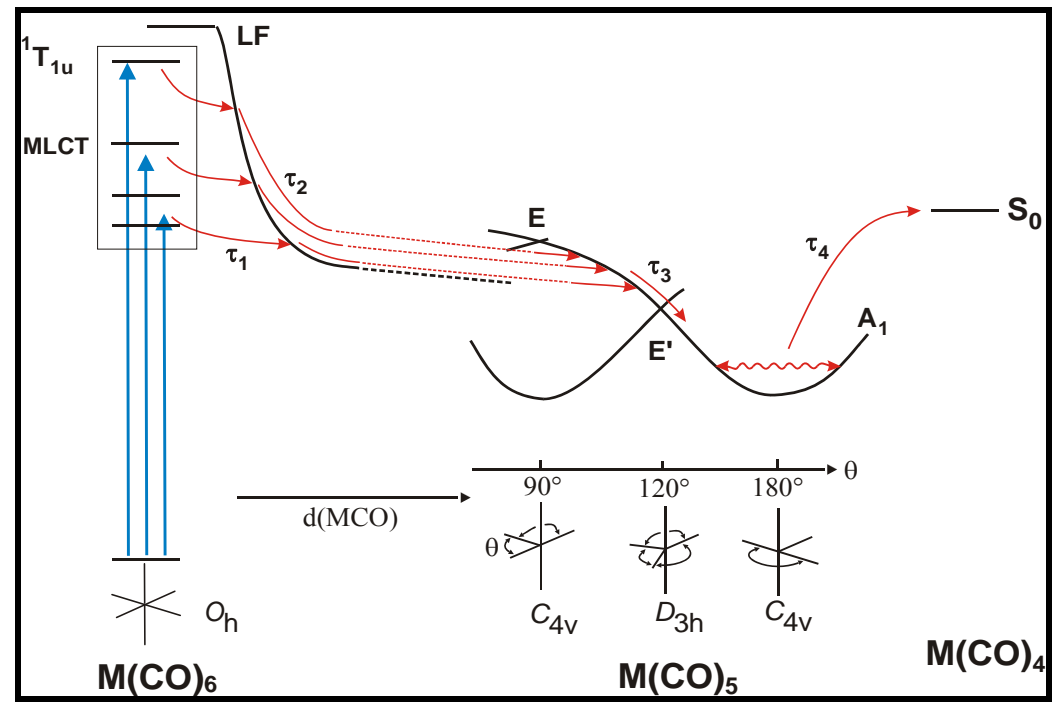

Fig. 6.4.1.1 Schematic potentials and dynamics, using three different pump wavelengths (270, 318 and 345 $\mathrm{nm}$ ), which initially excite MLCT states $\left(\mathrm{T}_{1 \mathrm{u}}\right.$ with $270 \mathrm{~nm}$ ). The initial relaxation (during slightly different times $\tau_{1}$ ) follows $\mathrm{Cr}-\mathrm{C}$ stretch and JT active coordinates and then the wave packet crosses over to the lowest LF surface. On this surface, the trajectories are probably still separate (during $\tau_{2}$ ), merging only above the conical intersection $(\mathrm{CI})$ in the dissociation product $\mathrm{Cr}(\mathrm{CO})_{5}$. During $\tau_{2}$, the $\mathrm{Cr}-\mathrm{C}$ distance increases much less than indicated in the figure (broken lines) for clarity. After passing the CI (within $\tau_{3}$ ), the wave packet oscillates in the $\mathrm{C}_{4 \mathrm{v}}$ minima along the pseudorotation coordinates indicated at the bottom; there are three symmetryequivalent such minima around each $\mathrm{D}_{3 \mathrm{~h}} \mathrm{CI}$ (see the drawing in [189]). The $\mathrm{Cr}-\mathrm{C}$ stretch oscillation (observed in all $\mathrm{L}_{i}$ ) is not indicated. Further dissociation to $\mathrm{Cr}(\mathrm{CO})_{4}$ (within $\tau_{4}$ ) is promoted by excess energy.

For assignment of the $\tau_{i}$ and ${ }^{m} \sigma_{i}$ and hence the identification of the $\mathrm{L}_{i}$, we keep in mind that the degree of fragmentation increases with increasing $i$ as has already been explained. Therefore the intensity of the parent ion $\mathrm{Cr}(\mathrm{CO})_{6}{ }^{+}$is largest in the Franck-Condon region $(i=1)$, whereas the final product $\left(\mathrm{L}_{5}\right)$ (with "infinite" (>500 ps) lifetime) gives rise to small-mass signals only. In the given case of $\mathrm{Cr}(\mathrm{CO})_{6}$, the conclusions drawn from the time-resolved experiments (at $267 \mathrm{~nm}$ [189]) and calculations [188, 192] point to the assignment, in particular of the later steps. The final time constant $\tau_{4}$ (for the process $\mathrm{L}_{4} \rightarrow \mathrm{L}_{5}$ ) of around 1 ps slightly depends on the excitation wavelength (Table 6.3.2.2) and thus on the excess energy in $\mathrm{L}_{4}$; hence it is probably a ground-state process, probably $\mathrm{Cr}(\mathrm{CO})_{5} \rightarrow \mathrm{Cr}(\mathrm{CO})_{4}$, as indicated in Fig. 6.4.1.1. In fact, this process is observed in the gas phase but suppressed in solution [193-196] and $\tau_{4}$ is the only time, which is long enough to allow cooling by the solvent to prevent this reaction. $\mathrm{L}_{4}$ can be identified with (the ground state of) $\mathrm{Cr}(\mathrm{CO})_{5}$ (and not a smaller fragment) also by 
the oscillations observed there: they have the same frequency in all signals $\mathrm{M}(\mathrm{CO})_{m}{ }^{+}$with $m=$ $0 \ldots 5$ [189] $\left(m=0 . .4\right.$ for $\mathrm{M}=\mathrm{Cr}$; but note that the ion $\mathrm{Cr}(\mathrm{CO})_{5}{ }^{+}$is lost already with very minor excess energy due to its extremely small dissociation barrier [189]); hence they are all due to a single neutral species $\mathrm{M}(\mathrm{CO})_{m}$ with $m \geq 5$. Actually, $m=6$ can be excluded, because it is known that it does dissociate.

The preceding step $\left(\tau_{3}\right)$ was already previously [189] assigned to the lifetime of the first excited singlet state $\mathrm{S}_{1}$ of $\mathrm{Cr}(\mathrm{CO})_{5}$, which is depleted to $\mathrm{S}_{0}$ via a Jahn-Teller induced conical intersection (Fig. 6.4.1.1). The idea of initial population of $S_{1}$ was based on a correlation argument and the observation of luminescence after dissociation of nickel carbonyl. This assumption leads to an easy rationalization of the oscillations in $\mathrm{L}_{4}$ : they are driven by the slope on leaving the conical intersection; the vibrational coordinate is hence expected - and found to coincide with the Jahn-Teller (JT) active coordinates (see below). These processes have all been confirmed by calculation $[188,192]$. The preceding step $\left(\tau_{2}\right)$ must then represent the dissociation itself and $\tau_{1}$ must be the relaxation out of the Franck-Condon region in the intact molecule.

The oscillations can be easily identified by their frequencies: the higher wavenumbers (350-450 $\mathrm{cm}^{-1}$, not resolved in [189]) are in the range where only MC stretch vibrations are observed in metal carbonyls (see, e.g. [197]). Selection rules suggest that it is the totally symmetric one. The lower wavenumber $\left(80-95 \mathrm{~cm}^{-1}\right)$, observed only in the dissociation product $\mathrm{Cr}(\mathrm{CO})_{5}$, is in the range of $\mathrm{CO}-\mathrm{M}-\mathrm{CO}$ bending vibrations [197]. In $D_{3 \mathrm{~h}}$ (symmetry at the conical intersection, Fig. 6.4.1.1) there are only two such vibrations (both degenerate, $e^{\prime}$, one involving the axial and the other the equatorial ligands) and they are JT active. In the symmetry $\left(C_{4 \mathrm{v}}\right)$ of the $\mathrm{S}_{0}$ minima (Fig. 6.4.1.1) they decompose into $e+a_{1}+b_{2}$, the degenerate one involving the axial ligand and the latter two corresponding to pseudorotation of the ligands in the basal plane (Fig. 6.4.1.1). It was suggested in [189] and confirmed by calculation [188, 192] that the observed oscillation just corresponds to this pseudorotation in $\mathrm{M}(\mathrm{CO})_{5}$ (Fig. 6.4.1.1); it is expected to be the totally symmetric component $\left(a_{1}\right.$ in $\left.C_{4 \mathrm{v}}\right)$ of it. Obviously it is driven by the slope in the pseudorotation direction on leaving the conical intersection (Fig. 6.4.1.1).

Whereas the assignments so far apply to $267 \mathrm{~nm}$ excitation [189], it is important to note that it can be applied with practically no change (except minor variations in the lifetimes) to 
the other investigation wavelengths too. Hence, we can immediately conclude that the states involved at the other wavelengths must be very similar (i.e. in particular no triplets).

\subsubsection{The reaction path of the dissociation}

Since the work of the Pierloot and Baerends groups [179, 180, 184, 185], there is agreement that most LF states lie above the MLCT states in $\mathrm{M}(\mathrm{CO})_{6}$. Practically only the latter are excited in the near UV, also because the LF states (involving g-g excitation), e.g. near $250 \mathrm{~nm}$, have much smaller oscillator strength than the MLCT states according to [198]. The - repulsive - LF potentials energetically steeply fall down along the metal-carbon stretch coordinate, whereas the MLCT states are primarily not dissociative, since they correlate with chargeseparated products. However, an avoided crossing with the LF curves can give rise to a minor or even vanishing barrier, over which the initial MLCT population can reach the dissociative curve $[179,180,185]$. The dissociation is hence not direct, but rather requires a crossing-over to an LF potential surface. This seems to be in full agreement with the observation that a relaxation step (within $\tau_{1}$ ) precedes the actual dissociation (within $\tau_{2}$ ).

A closer investigation of the energies along the $\mathrm{M}-\mathrm{C}$ stretch coordinate by time-dependent density functional theory suggested, however, that only one repulsive curve results from this mechanism, starting from one (or two) of the lowest MLCT states $\left(\mathrm{T}_{2 \mathrm{u}}\right.$, which can mix with $E_{u}$ ), whereas the other MLCT states seemed to be bound [179, 180, 185]. How does then, for instance, the $\mathrm{T}_{1 \mathrm{u}}$ population (excited at $270-282 \mathrm{~nm}$ ) find the repulsive curve within $12.5 \mathrm{fs}$ ? This short time obviously requires a very rapid acceleration and hence a very steep initial down-slope. In $[189,190]$ it was suggested that such slope exists along Jahn-Teller (JT) active coordinates, while the JT-induced crossings can provide an ultrafast path, for example, from $T_{1 u}$ down to $T_{2 u}$, from where population flows to the repulsive LF state $[189,190]$. However, if the time $\tau_{1}$ represents the $T_{1 u} \rightarrow T_{2 u}$ relaxation, this step should not exist on direct excitation of the $\mathrm{T}_{2 \mathrm{u}}$ state at 318 or $345 \mathrm{~nm}$ (whatever is the spectral assignment), which is in contrast to our observation (Table 6.3.2.1). A direct and fast path seems, therefore, to exist from every MLCT state to a repulsive LF state and we suggest here that $\tau_{1}$ is the time constant for MLCT $\rightarrow$ LF relaxation in all cases, as indicated in Fig. 6.4.1.1.

In density functional theory the lowest LF state $\left(\mathrm{T}_{1 \mathrm{~g}}\right)$ is below the $\mathrm{T}_{1 \mathrm{u}}$ state [185], but in the more accurate CASPT2 calculations, $\mathrm{T}_{1 \mathrm{~g}}$ is slightly above $\mathrm{T}_{1 \mathrm{u}}[184,187]$; both states split along the $\mathrm{MC}$ stretch coordinate to an $\mathrm{E}$ and an $\mathrm{A}_{1}$ state (in $C_{4 \mathrm{v}}$ ), which will lead to an avoided 
crossing similar as with the lower $\mathrm{T}_{2 \mathrm{u}}$ state. It is thus suggested (explained in detail in [8]) that there is a direct MLCT $\rightarrow$ LF relaxation from $\mathrm{T}_{1 \mathrm{u}}$ via an avoided crossing along M-CO stretching, while from the higher $\mathrm{T}_{1 \mathrm{u}}$ state (excited at $224 \mathrm{~nm}$ ), the same LF state can be reached in a different direction, namely along JT active coordinates. This path resembles the suggestion in $[189,190]$, but now represents a direct MLCT $\rightarrow$ LF relaxation on $T_{1 u}$ excitation $(270-290$ or $224 \mathrm{~nm}$ ), without the intact molecule reaching $\mathrm{S}_{1}$ before. It should further be noted that distortion along $\mathrm{JT}$ active coordinates ( $e_{\mathrm{g}} \mathrm{MC}$ stretching and $t_{2 \mathrm{~g}} \mathrm{MCO}$ bending) mixes the different MLCT states quite strongly too. This is implied by the not very different oscillator strengths of allowed and forbidden bands (Fig. 6.2.2), indicating strong intensity borrowing. Strong vibronic interaction points to the neighbourhood of a conical intersection $(\mathrm{CI})$ and a $\mathrm{CI}$ can usually provide an ultrafast path from a (in this case: slightly) higher to a lower state, e.g. from $\mathrm{A}_{2 \mathrm{u}}$ to $\mathrm{T}_{2 \mathrm{u}}$.

Moreover, (unlike in a statistical model, where the lifetimes would monotonically decrease with increasing excess energy) the wavelength dependence of the time constants is nonmonotonic (Table 6.3.2.1,3) and the $\tau_{1}$ seem to be characteristic of every initially excited state. This can be explained if one assumes that they are controlled by the initial accelerations and slopes (and additionally by the height or absence of a barrier resulting from an avoided crossing). By far the longest $\tau_{1}$ is observed at $162 \mathrm{~nm}$, which probably excites a Rydberg state [8]. It is not expected to be repulsive (its potential resembles that of a $\mathrm{Cr}(\mathrm{CO})_{6}{ }^{+}$ion in the $\mathrm{T}_{2 \mathrm{~g}}$ ground state) and the repulsive valence states are much lower in energy; an intersection or avoided crossing between the two types of potentials may therefore be far from the Rydberg minimum and may involve some activation energy.

Information on the initial coordinates can be derived from resonance Raman spectra. With pump wavelengths between 355 and $213 \mathrm{~nm}$, activity was found in JT active $e_{\mathrm{g}}$ and $t_{2 \mathrm{~g}}$ modes in $\mathrm{Cr}(\mathrm{CO})_{6}$ and $\mathrm{W}(\mathrm{CO})_{6}[199-201]$ (confirming the suggestion, that they are components of the direction of the initial slope), while at $266 \mathrm{~nm}$, also $t_{1 \mathrm{u}}$ and $t_{2 \mathrm{u}}$ overtones were found and interpreted as an indication of vibronic mixing of LF and MLCT states [199-201]. A remarkable finding is that all the vibrational progressions were found to be very short [199-201] (up to the first overtone; only traces of higher ones were detected in [200]). Although this was interpreted in [199] in terms of an upper-state potential that is nearly not shifted versus the equilibrium geometry, it is incompatible with the ultrashort $\tau_{1}$ values found here, implying a 
very fast acceleration and a steep initial slope. We instead infer from the short progressions that the wave packet leaves the initially excited state very early (within $\tau_{1}$ ) to a potential surface (such as an LF surface), from where transition to $S_{0}$ involves a much smaller transition moment. To explain the short progressions, Adelman and Gerrity [199] also suggested intersystem crossing. This was, however, excluded in [189], because all time constants were found to be longer with heavier metal hexacarbonyls.

Furthermore, photofragment spectroscopy supports a specific JT active mode $\left(t_{2 \mathrm{~g}}\right.$, which is an $\mathrm{MCO}$ bending coordinate), since rotationally excited $\mathrm{CO}$ was found after photodissociation of $\mathrm{Cr}(\mathrm{CO})_{6}$ [202-204]. This observation indicates a bent MCO structure before dissociation, so that the recoil can excite rotation. Such a bending can be provided by a $t_{2 \mathrm{~g}}$ deformation [189].

The time $\tau_{1}$ is amazingly short $(12.5 \mathrm{fs}$ with $270 \mathrm{~nm})$. Travelling from any point on the slope of a bound potential (for example along an $\mathrm{MC}$ stretch coordinate with vibrational wavenumber of $400 \mathrm{~cm}^{-1}$ ) to its minimum (or a point close-by, where it might find an outlet - a CI or an avoided crossing - to another potential), a wave packet needs one quarter of a period, 20 fs. At the Franck-Condon point with its $O_{\mathrm{h}}$ symmetry, the only coordinates with nonzero slope are either totally symmetric $\left(a_{1 \mathrm{~g}}\right)$ or JT active. Along the $a_{1 \mathrm{~g}} \mathrm{MC}$ stretching, six MC bonds are extended in phase and the potential in this direction is bound; its curvature corresponds to a vibrational wavenumber of $360 \mathrm{~cm}^{-1}$ (period $93 \mathrm{fs}$ ). Reaching the minimum would thus take $23 \mathrm{fs}$, longer than observed $(12.5 \mathrm{fs})$ in $\mathrm{Cr}(\mathrm{CO})_{6}$. For the JT active MC stretch mode $\left(e_{\mathrm{g}}\right)$ a similar wavenumber is expected, judging from the ground state [197], whereas a higher frequency is expected for the JT active MCO bending $\left(t_{2 \mathrm{~g}}, 532 \mathrm{~cm}^{-1}\right.$ in $\mathrm{S}_{0}$ [197], 15 fs for a quarter of a period). Therefore, it is suggested that excursion along this direction significantly contributes to initial acceleration and transition to the LF state (in agreement with the evidence from CO rotational excitation in photofragement spectroscopy and the Raman spectra). Later on, an ungerade direction such as $t_{1 u}$ must mix in, since gerade coordinates would not lead to dissociation, because they involve more than one ligand. (A local MC stretch is a superposition of $t_{1 \mathrm{u}}$ with $e_{\mathrm{g}}$ and $a_{1 \mathrm{~g}} \mathrm{MC}$ stretch coordinates.) $u$-Type distortion breaks the inversion symmetry, which is also necessary to permit transition from an MLCT state ( $u$ symmetry) to an LF potential ( $g$ symmetry). 
The dissociation takes place after crossing over to the lowest LF potential surface (symmetry type $T_{1 \mathrm{~g}}$ in $O_{\mathrm{h}}$ ); the $\mathrm{MC}$ bond is extended there and the state splits. The wave packet flows to the lower one ( $E$ symmetry in $C_{4 \mathrm{v}}$ ) and within $\tau_{2}$ arrives at the $\mathrm{S}_{1}$ state of $E$ symmetry of $\mathrm{Cr}(\mathrm{CO})_{5}$. The dissociation is remarkably fast $\left(\tau_{2}=18-22 \mathrm{fs}\right.$, Table 6.3.2.1), probably the fastest measured to date by time resolution. $\mathrm{Cr}(\mathrm{CO})_{5}$ is formed (Fig. 6.2.1) in the $\mathrm{S}_{1}$ state $(E$ in $C_{4 \mathrm{v}}$ ) and relaxes within $\tau_{3}$ to $\mathrm{S}_{0}$ (Fig. 6.4.1.1). In [8] it was argued that all the paths starting from the different initial states (including those excited at the longer wavelength region) go down on the same LF surface and reach the excited state of the pentacarbonyl, although with longer wavelengths the trajectories lie deeper in the valleys leading closer to the $\mathrm{CI}$ in $\mathrm{Cr}(\mathrm{CO})_{5}$ (Fig. 6.4.1.1). This is probably why the $\mathrm{S}_{1}$ lifetimes $\left(\tau_{3}\right)$ of $\mathrm{Cr}(\mathrm{CO})_{5}$ (Table 6.3.2.1) decrease with longer pump wavelength, since the travelling distance to the $\mathrm{CI}$ is shorter in this case. Lifetimes that are shorter with less excess energy would probably be hard to rationalize in a different way.

The short $\tau_{3}$ (Table 6.3.2.1) implies a barrierless continuous path down to $\mathrm{S}_{0}$ of $\mathrm{Cr}(\mathrm{CO})_{5}$. $\mathrm{A}$ JT-induced conical intersection (CI) is expected in the trigonal-bipyramidal molecular structure (symmetry $D_{3 \mathrm{~h}}$ ) of $\mathrm{Cr}(\mathrm{CO})_{5}$ and the JT active coordinates correspond to CMC bending (symmetry $e^{\prime}$ in $D_{3 \mathrm{~h}}, a_{1}+b_{2}$ in $C_{4 \mathrm{v}}$ ), which basically exchange the ligand vacancy (in squarepyramidal geometry) with an adjacent $\mathrm{CO}$ group (pseudorotation, Fig. 6.4.1.1). After passing through the $\mathrm{CI}$, the wave packet oscillates in $\mathrm{S}_{0}$ (i.e. in $\mathrm{L}_{4}$ ) along the pseudrotation coordinate with a period of $350 \mathrm{fs}$. In a quarter of this period (in $88 \mathrm{fs}$ ) the wave packet should travel from the point of maximum amplitude (turning point) to the minimum. However, $\tau_{3}$ is much shorter (40-25 fs). It is suggested that this is because the wave packet arrives in $\mathrm{S}_{1}$ not at the FC point (i.e. above the $\mathrm{S}_{0}$ minimum) but closer to the CI (as indicated in Fig. 6.4.1.1), having, additionally, a $b_{2}$ momentum acquired before (by the JT effect on the repulsive curve). The same suggestion was used above to rationalize the unusual excess-energy dependence of $\tau_{3}$.

The time $\tau_{4} \approx 1$ ps for further dissociation from the hot $\mathrm{S}_{0}$ of $\mathrm{Cr}(\mathrm{CO})_{5}$ is shorter than statistically expected with the excess energy from 270-nm dissociation [189]. Apparently, compared to equipartition, a larger fraction of the energy seems to be in suitable stretch coordinates. A nonstatistical energy distribution is also required to explain the coherent oscillations 
observed. The fact that $\tau_{4}$ only increases from 0.93 to $1.5 \mathrm{ps}$ (Table 6.3.2.1) on decreasing the excess energy by $1.0 \mathrm{eV}$ (increasing the pump wavelength from 270 to $345 \mathrm{~nm}$ ) is also consistent with this non-equipartition situation.

In $[189,190]$ coherent oscillations were found after dissociation, in the ground singlet state of $\mathrm{M}(\mathrm{CO})_{5}$ (wavenumbers 95,84 and $80 \mathrm{~cm}^{-1}$ for $\mathrm{M}=\mathrm{Cr}$, Mo and $\mathrm{W}$, respectively). They were identified with the pseudorotation vibrations in a $C_{4 \mathrm{v}}$ minimum, obviously stimulated by the slope down from the conical intersection of $D_{3 \mathrm{~h}}$ symmetry, as also confirmed by the calculations by Paterson, Worth et al. [188, 192]. They are now found again in [8]. Besides MCO bending, our time resolution ( $\sim 30 \mathrm{fs})$ now also allows the detection of a high-frequency oscillation in $\mathrm{L}_{4}$ (around $400 \mathrm{~cm}^{-1}$ ) and the preceding locations (Table 6.3.2.2). It is assigned to the totally symmetric one ( $a_{1 \mathrm{~g}}$ "breathing" in $O_{\mathrm{h}}, a_{1}$ in $C_{4 \mathrm{v}}$ ), because from the antisymmetric vibrations one in general only observes overtones [79]. This vibration is already found in the FC region $\left(\mathrm{L}_{1}\right)$ of $\mathrm{Cr}(\mathrm{CO})_{6}$ with longer-wavelength excitation (and of $\mathrm{Mo}(\mathrm{CO})_{6}$ excited at 270 $\mathrm{nm}\left(310 \mathrm{~cm}^{-1}\right)$ [8]). Obviously with $270 \mathrm{~nm}$, in this molecule $\tau_{1}(12.5 \mathrm{fs})$ is a fraction of the period (80-100 fs), too small to allow detection of such a modulation. Indeed this vibration was also detected in static spectroscopy in a cryogenic matrix as vibrational structure in the $\mathrm{T}_{1 \mathrm{u}}$ band of the molybdenum and tungsten carbonyls (wavenumbers $\approx 350$ and $\approx 400 \mathrm{~cm}^{-1}$, respectively) but not in chromium carbonyl [205], certainly for the same reason.

The MC stretch vibration is seen as a coherent oscillation in the locations $\mathrm{L}_{2}-\mathrm{L}_{4}$ on the potentials of $\mathrm{Cr}(\mathrm{CO})_{6}$ (with different frequencies), although a down-slope along this direction was postulated for $\mathrm{L}_{1}$, and although $\mathrm{L}_{2}$ was described above as a potential that is strongly repulsive along the MC stretch coordinate. However, the observed vibration takes place along an $a_{1 \mathrm{~g}}$ direction, involving in-phase stretching of six MC bonds, which does not lead to dissociation. The symmetric vibration is schematically illustrated in Fig. 6.4.1.2, which also shows two of the channels for dissociation; the latter follow the bottom of the valley, whereas the vibration is orthogonal to this direction. This is similar as the coherent $\mathrm{HgI}$ oscillation found in the early time of femtosecond chemistry by the Zewail group in $\mathrm{HgI}_{2}$ dissociation [206, 207]. The vibration also survives in the following locations and is monitored for the chromium carbonyl system down to $\mathrm{S}_{0}$ of the dissociation product (i.e. $\mathrm{L}_{4}$ ). On a change of the electronic state, it substantially changes its frequency (by 20\%, Table 6.3.2.2). Normally such a change leads to rapid dephasing of the coherent motion. However, all the relaxations take 
place in times $\left(\tau_{1}-\tau_{3}\right)$ shorter than a period, so that dephasing cannot fully develop. But such an effect can be the reason, why in $\mathrm{L}_{4}$ (ground state of $\mathrm{Cr}(\mathrm{CO})_{5}$ ) the dephasing time of this vibration (165-270 fs) is much shorter than that of the lower-frequency vibration ( $>1 \mathrm{ps})$, which is only excited on the lower surface: the $a_{1}$ vibration changes by $20 \%$ (from 450 to 350 $\left.\mathrm{cm}^{-1}\right)$ on $\mathrm{S}_{1} \rightarrow \mathrm{S}_{0}\left(\mathrm{~L}_{3} \rightarrow \mathrm{L}_{4}\right)$ relaxation, so that after a small multiple of the vibrational period (74 fs in $\mathrm{S}_{1}$ ) the two vibrations will be out of phase.

The phases $\varphi$ of the oscillations, reported in Table 6.3.2.2, should monotonically increase within the model that the oscillations simply propagate from location to location. The measured data are consistent with a monotonic increase, if one takes into account that $\varphi$ is defined only up to modulo $2 \pi$. An alternative to propagating oscillations, excited in the FC region, postulates restimulation of $\mathrm{MC}$ stretch vibration on each crossing-over to a new electronic state, by which the MC vibrational frequency or the bond length is suddenly changed. In fact, the $\mathrm{MC}$ stretch vibration was found oscillating in $\mathrm{S}_{0}$ of $\mathrm{Cr}(\mathrm{CO})_{5}$ in the quantum dynamics simulation [192], that started from the $\mathrm{S}_{1}$ of this molecule (i.e. not from the FC region of $\left.\mathrm{Cr}(\mathrm{CO})_{6}\right)$.

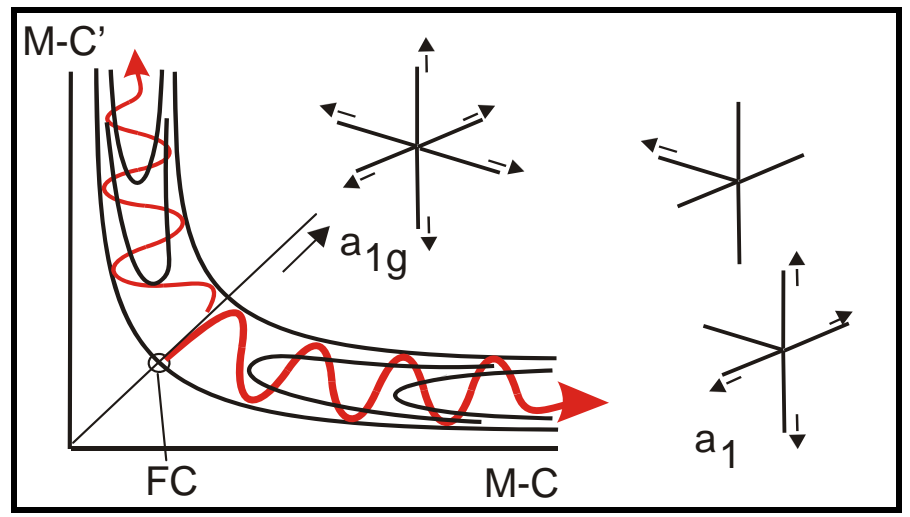

Fig. 6.4.1.2 The symmetric $\mathrm{Cr}-\mathrm{CO}$ stretch vibration $\left(a_{1 \mathrm{~g}}\right)$, transforming along localized $\mathrm{Cr}-\mathrm{CO}$ dissociation coordinates into two $a_{1}$ vibrations in $\mathrm{Cr}(\mathrm{CO})_{5}$. Also shown are two of the channels for dissociation.

\subsection{Conclusions}

The time resolution of about $30 \mathrm{fs}$ achieved in the present study of the dissociation of $\mathrm{Cr}(\mathrm{CO})_{6}$ (40 fs with the $345-\mathrm{nm}$ pulses) allowed to determine three steps during the relaxation process, $\tau_{1}=12.5 \mathrm{fs}, \tau_{2}=18 \mathrm{fs}$ and $\tau_{3}=40 \mathrm{fs}$ (excitation at $270 \mathrm{~nm}$; $\tau_{1}$ confirmed with 10 -fs resolution [4]), as well as the lifetime of the product $\mathrm{Cr}(\mathrm{CO})_{5}$ in the ground state, $\tau_{4}=1 \mathrm{ps}$. The sup- 
posed second relaxation step in the intact $\mathrm{Cr}(\mathrm{CO})_{6}$ in [189] (with time constant $\tau_{2 \text { old }}=10-20$ fs) is now found to contain a periodic modulation and not be purely exponential. It corresponds to a totally symmetric $\mathrm{Cr}-\mathrm{CO}$ stretch vibration, taking place during elimination of one $\mathrm{CO}$ ligand. It is already excited in the Franck-Condon region by the pump laser and seems to survive over four electronic levels down to the ground state of the dissociation product, although restimulation by a changed $\mathrm{Cr}-\mathrm{CO}$ distance or slope on transition to a new electronic state may also contribute. Also found was a lower-frequency oscillation that appears after the molecule reaches a minimum of $C_{4 \mathrm{v}}$ symmetry in $\mathrm{Cr}(\mathrm{CO})_{5}$. It is assigned to a totally symmetric $\mathrm{C}-\mathrm{Cr}-\mathrm{C}$ bending vibration associated with pseudorotation; it is stimulated only later, by the slope on entering this potential well from the $\mathrm{S}_{1} / \mathrm{S}_{0}$ conical intersection with trigonalbipyramidal molecule geometry. Such chemically induced vibrations (as opposed to vibrations excited optically in the Franck-Condon region) can be very useful to find out the directions of slopes and hence information about the reaction coordinates. A strong support for the nature of excitation of this vibration is the recent calculations [188, 192], as well as chemically induced vibrations found in other molecular systems [208].

The process of the elimination of one $\mathrm{CO}$ and relaxation of $\mathrm{Cr}(\mathrm{CO})_{5}$ in its ground state consists of three steps (Fig. 6.4.1.1), while the actual dissociation is completed within $\tau_{3}=18 \ldots 22$ fs. As concluded by exciting different initial states (in the region $270-345$ $\mathrm{nm}$ ) for all MLCT states there is a fast path along JT-active and/or $\mathrm{Cr}-\mathrm{CO}$ stretch coordinates directly to the repulsive LF potential (within $\tau_{1}$ ), which then leads to dissociation within $\tau_{2}$ with $\mathrm{Cr}(\mathrm{CO})_{5}$ being produced in the $\mathrm{S}_{1}$ excited state; the different trajectories merge in the product molecule at (or just above) the $\mathrm{S}_{1} / \mathrm{S}_{0}$ conical intersection. These observations modify the suggested dynamics of the dissociation in [189], where after excitation at $270 \mathrm{~nm}$ the molecule first relaxed to one of the lowest singlet states (to $\mathrm{T}_{2 \mathrm{u}}$ within $\tau_{1}$ ) via avoided crossings induced by JT distortions of the potentials and then reached a repulsive LF state (within $\tau_{2 \text { old }}$ ). Within the "pathway approach" (Chapter 3 ) the choice between the two relaxation paths (direct MLCT $\rightarrow$ LF crossing-over versus a preceding down-relaxation to $T_{2 u}$ ) is probably decided by the relative slopes and intersections between the potential surfaces. This is in contrast to the traditional approach which considers densities of energy levels and assumes vertical relaxation among excited states, leading to equipartition of the energetically 
accessible states. Such a statistical process is further excluded by the coherent oscillations observed here.

Moreover, the wavelength-independent dissociation mechanism implies that the longwavelength wing of the $\mathrm{Cr}(\mathrm{CO})_{6}$ spectra do not reflect singlet-triplet transitions, in contrast to the early [178, 209] and a very recent [187] suggestion and the assignment in textbooks [182, 191]. One can also rule out intersystem crossing that would produce triplets from initial singlets still in the intact molecule $([182,191])$. This was already excluded in [189] as all the measured time constants were longer in the heavier $\mathrm{M}(\mathrm{CO})_{6}$, whereas spin-orbit coupling would result in the opposite trend. From an experiment with much less time resolution, Joly and Nelson already concluded that dissociation takes place in the singlet manifold [210]. The conclusion that the triplets are not involved has chemical implications: a singlet $\mathrm{Cr}(\mathrm{CO})_{5}$ can easily undergo reactions such as insertions into $\mathrm{CH}$ bonds (analogous molecules are therefore in industrial use for catalysis) [181], whereas a triplet would only undergo radical reactions.

Although the dissociation of $\mathrm{Cr}(\mathrm{CO})_{6}$ is indirect (i.e. the repulsive potential is reached only after a preceding relaxation step), its observed duration (whether one takes $\tau_{1}+\tau_{2}$ or only $\tau_{2}$ ) is the shortest time for dissociation observed so far in time-resolved experiments. 


\section{APPENDIX}

\section{Derivation of the Equation 3.4.3.1 of Chapter 3, section 3.4.3}

We assume that our system is described by the ground $|1\rangle$, first excited $|2\rangle$ state and the ionization continuum. The $N$ molecules originally in the ground state interact with the pump laser pulse $I_{\mathrm{pu}}(t, r)=I_{\mathrm{pu}}^{0} \exp \left[-\left(t / \tau_{\mathrm{pu}}\right)^{2}\right] \exp \left[-\left(r / 2 w_{\mathrm{pu}}\right)^{2}\right]$ and the number of molecules excited in $|2\rangle$ per unit time is $n_{2}=\sigma_{\mathrm{pu}} F_{\mathrm{pu}} N ; \sigma_{\mathrm{pu}}$ is the cross section for the absorption of one pump photon, $F_{\mathrm{pu}}=I_{\mathrm{pu}} / \hbar \omega_{\mathrm{pu}}$ is the number of photons per unit area and unit time, $\omega_{\mathrm{pu}}$ is the carrier frequency of the pump pulse, $\tau_{\mathrm{pu}}$ its temporal Gaussian width and $w_{\mathrm{pu}}$ the beam cross section in the focal plane. $\tau_{\mathrm{pu}}$ is connected to the full-width-at-half-maximum (FWHM) by $t_{\mathrm{FWHM}}=2 \sqrt{\ln 2} \tau_{\mathrm{pu}}$. The peak of the pump pulse is at time $t=0$.

The probe pulse $I_{\mathrm{pr}}(t, r)=I_{\mathrm{pr}}^{0} \exp \left[-\left((t-\Delta t) / \tau_{\mathrm{pr}}\right)^{2}\right] \exp \left[-\left(r / 2 w_{\mathrm{pr}}\right)^{2}\right]$ arrives after some delay $\Delta t$ from the pump and after interaction with the population in state $|2\rangle$ ionizes $n_{3}=\sigma_{\mathrm{pr}}^{(l)} F_{\mathrm{pr}}^{l} n_{2}$ molecules per unit time; $\sigma_{\mathrm{pr}}^{(l)}$ is the cross section for absorption of $l$ probe photons and $F_{\mathrm{pr}}=I_{\mathrm{pr}} / \hbar \omega_{\mathrm{pr}}, \omega_{\mathrm{pr}}, \tau_{\mathrm{pr}}$ and $w_{\mathrm{pr}}$ are defined as the respective parameters of the pump pulse.

The transition rate from the ground to the first excited state is $W_{\mathrm{pu}}=\sigma_{\mathrm{pu}} F_{\mathrm{pu}}$ and the transition rate for ionization is $W_{\mathrm{pr}}=\sigma_{\mathrm{pr}}^{(l)} F_{\mathrm{pr}}^{l}$. Assuming that the population in $|2\rangle$ relaxes with rate $k$, the rate equations for this 3-level system in the unsaturated regime, i.e. when there is not considerable depletion of the ground state population and $n_{2}, n_{3}<<n_{1}$ are

$$
\begin{aligned}
\frac{\mathrm{d} n_{1}}{\mathrm{~d} t} & =0 \Rightarrow n_{1}=N \\
\frac{\mathrm{d} n_{2}}{\mathrm{~d} t} & =W_{\mathrm{pu}}(t) n_{1}-k n_{2} \\
\frac{\mathrm{d} n_{3}}{\mathrm{~d} t} & =W_{\mathrm{pr}}(t-\Delta t) n_{2}
\end{aligned}
$$


$n_{1}(t)$ is the population of the ground state and $n_{1}(t \rightarrow-\infty)=N, n_{2}(t \rightarrow-\infty)=0=$ $n_{3}(t \rightarrow-\infty)$. Equation (1) may be solved using the integrating factor exp $(k t)$, yielding

$$
\begin{aligned}
n_{2}(t) & =N \int_{-\infty}^{t} \exp [-k(t-x)] W_{\mathrm{pu}}(x) d x \\
& =\mathrm{A} \times \exp (-k t) \times\left(1+\operatorname{erf}\left[\frac{1}{\sqrt{2}}\left(t / \tau_{\mathrm{pu}}-\tau_{\mathrm{pu}} k\right)\right]\right)
\end{aligned}
$$

where A is a constant proportional to $\sigma_{\mathrm{pu}}$ and $\operatorname{erf}(x)=\frac{2}{\sqrt{\pi}} \int_{0}^{x} \exp \left(-y^{2}\right) d y$ is the error function. Integration of equation (2) leads to an expression for $n_{3}(t, \Delta t)$ and after substituting $n_{2}$ from (3), the ion signal $\left(n_{3}\right)$ as a function of the time delay is

$$
\begin{aligned}
S_{1}(\Delta t) & =\int_{-\infty}^{\infty} W_{\mathrm{pr}}(t-\Delta t)\left\{\int_{-\infty}^{t} N \exp [-k(t-x)] W_{\mathrm{pu}}(x) d x\right\} d t \\
& =\int_{-\infty}^{\infty} \int_{-\infty}^{t} W_{\mathrm{pr}}(t-\Delta t) N \exp [-k(t-x)] W_{\mathrm{pu}}(x) d x d t
\end{aligned}
$$

A change of variable $s=x-t+\Delta t$ [77] leads to

$$
\begin{aligned}
S_{1}(\Delta t) & =\int_{-\infty}^{\infty} \int_{-\infty}^{\Delta t} W_{\mathrm{pr}}(t-\Delta t) N \exp [-k(\Delta t-s)] W_{\mathrm{pu}}(s+t-\Delta t) d s d t \\
& =\int_{-\infty}^{\Delta t} \int_{-\infty}^{\infty} W_{\mathrm{pr}}(t-\Delta t) N \exp [-k(\Delta t-s)] W_{\mathrm{pu}}(s+t-\Delta t) d t d s \\
& =\int_{-\infty}^{\Delta t} N \exp [-k(\Delta t-s)]\left\{\int_{-\infty}^{\infty} W_{\mathrm{pr}}(t-\Delta t) W_{\mathrm{pu}}(s+t-\Delta t) d t\right\} d s \\
& =\int_{-\infty}^{\Delta t} N \exp [-k(\Delta t-s)]\left\{\int_{-\infty}^{\infty} W_{\mathrm{pr}}(x-s) W_{\mathrm{pu}}(x) d x\right\} d s \\
& =\int_{-\infty}^{\Delta t} N \exp [-k(\Delta t-s)] F(s) d s
\end{aligned}
$$


$F(s)=\int_{-\infty}^{\infty} W_{\mathrm{pu}}(x) W_{\mathrm{pr}}(x-s) d x$ is the cross-correlation function of $W_{\mathrm{pu}}$ with $W_{\mathrm{pr}}$. Therefore $F(s)$ is directly proportional to the cross-correlation of the pump with the $l$ power of the probe pulse $R(s)=\int_{-\infty}^{\infty} I_{\mathrm{pu}}(x) I_{\mathrm{pr}}^{l}(x-s) d x$. After the integration in equation (4) we get:

$$
S_{1}(\Delta t)=\mathrm{B} \times \exp (-k \Delta t) \times\left(1+\operatorname{erf}\left[\frac{1}{\sqrt{2}}(\Delta t \gamma-k / 2 \gamma)\right]\right)
$$

where B is a constant proportional to $\sigma_{\mathrm{pr}}^{(l)}$ and $\gamma^{-1}=\sqrt{\frac{\tau_{\mathrm{pr}}^{2}+l \tau_{\mathrm{pu}}^{2}}{l}}=\tau_{\mathrm{cc}}$ is the Gaussian width of the cross-correlation $R(t)$.

If the population decay involves more than one location on the excited state, e.g. 2 , then the rate equations become (in the unsaturated regime)

$$
\begin{aligned}
& \frac{\mathrm{d} n_{1}}{\mathrm{~d} t}=0 \Rightarrow n_{1}=N \\
& \frac{\mathrm{d} n_{2}}{\mathrm{~d} t}=W_{\mathrm{pu}}(t) n_{1}-k n_{2} \\
& \frac{\mathrm{d} n_{3}}{\mathrm{~d} t}=k n_{2}-k_{1} n_{3} \\
& \frac{\mathrm{d} n_{4}}{\mathrm{~d} t}=W_{\mathrm{pr}}(t-\Delta t) n_{3}
\end{aligned}
$$

where $n_{2}$ is the population of the location $\mathrm{L}_{2}$ on the excited state which decays with rate $k$, while $n_{3}$ is the population of the location $\mathrm{L}_{3}$ on the excited state which rises with rate $k$ and decays with rate $k_{1}$. The probe pulse arrives after time delay $\Delta t$ from the pump and we suppose that it excites the population $n_{3}$ that that has flown from $\mathrm{L}_{2}$ to $\mathrm{L}_{3}$. From equation (5) we get the same $n_{2}$ as before and using this expression for equation (6) one obtains 


$$
\begin{aligned}
n_{3}(t) & =\mathrm{A}_{1} \exp (-k t)\left(1+\operatorname{erf}\left[\frac{1}{\sqrt{2}}\left(t / \tau_{\mathrm{pu}}-\tau_{\mathrm{pu}} k\right)\right]\right)+\mathrm{A}_{2} \exp \left(-k_{1} t\right)\left(1+\operatorname{erf}\left[\frac{1}{\sqrt{2}}\left(t / \tau_{\mathrm{pu}}-\tau_{\mathrm{pu}} k_{1}\right)\right]\right) \\
& =\left[k /\left(k_{1}-k\right)\right]\left\{N \int_{-\infty}^{t} \exp [-k(t-x)] W_{p u}(x) d x-N \int_{-\infty}^{t} \exp \left[-k_{1}(t-x) W_{p u}(x) d x\right]\right\}
\end{aligned}
$$

The signal $S_{2}(\Delta t)$ (which, in this case, results from ionization of the population $n_{3}$ is now

$$
\begin{aligned}
S_{2}(\Delta t) & =\left[k /\left(k_{1}-k\right)\right]\left\{\int_{-\infty}^{\Delta t} N \exp [-k(\Delta t-s)] F(s) d s+\int_{-\infty}^{\Delta t} N \exp \left[-k_{1}(\Delta t-s)\right] F(s) d s\right\} \\
& =\mathrm{B}_{1} \exp (-k \Delta t)\left(1+\operatorname{erf}\left[\frac{1}{\sqrt{2}}(\Delta t \gamma-k / 2 \gamma)\right]\right)+\mathrm{B}_{2} \exp \left(-k_{1} \Delta t\right)\left(1+\operatorname{erf}\left[\frac{1}{\sqrt{2}}\left(\Delta t-k_{1} / 2 \gamma\right)\right]\right)
\end{aligned}
$$

The total signal produced by ionization of the molecules from $\mathrm{L}_{1}$ and $\mathrm{L}_{2}$ will be of the form

$$
\begin{aligned}
\operatorname{Sig}(\Delta t) & =S_{1}(\Delta t)+S_{2}(\Delta t) \\
& =\mathrm{B} \times \exp (-k \Delta t) \times\left(1+\operatorname{erf}\left[\frac{1}{\sqrt{2}}(\Delta t \gamma-k / 2 \gamma)\right]\right) \\
& +\mathrm{B}_{1} \times \exp (-k \Delta t) \times\left(1+\operatorname{erf}\left[\frac{1}{\sqrt{2}}(\Delta t \gamma-k / 2 \gamma)\right]\right) \\
& +\mathrm{B}_{2} \times \exp \left(-k_{1} \Delta t\right) \times\left(1+\operatorname{erf}\left[\frac{1}{\sqrt{2}}\left(\Delta t \gamma-k_{1} / 2 \gamma\right)\right]\right)
\end{aligned}
$$

and more generally from more locations $\mathrm{L}_{i}$

$$
\operatorname{Sig}(\Delta t)=\sum_{n} \alpha_{n} \times \exp \left(-k_{n} \Delta t\right) \times\left(1+\operatorname{erf}\left[\frac{1}{\sqrt{2}}\left(\Delta t \gamma-k_{n} / 2 \gamma\right)\right]\right)
$$

where $\alpha_{n}=\sum \beta_{i} \times \sigma_{i}^{(l)}$ pr and $\sigma_{i}^{(l)}$ ir is the cross section for absorption ofl probe photons by the molecule in the location $\mathrm{L}_{i}$. 


\section{Summary}

In the present work the real-time dynamics of the light-induced (1) isomerization of $\mathrm{C}_{2} \mathrm{H}_{4},(2)$ ring-opening of $\mathrm{C}_{6} \mathrm{H}_{8}$ and (3) dissociation of chromium hexacarbonyl $\mathrm{Cr}(\mathrm{CO})_{6}$ were studied by temporal resolution in the 10 -fs time range, which is an order of magnitude better than before.

For this purpose ultrashort pulses in the UV (where these and many other molecules have their first absorption band) were developed by means of supercontinuum and third- and fifthharmonic generation. In a first step the 800-nm output from a Ti-sapphire laser was shortened: the pulses $(\sim 1 \mathrm{~mJ}, \approx 45 \mathrm{fs})$ are focused $(f=2 \mathrm{~m})$ into a (usually windowless) cell of 150 cm length with slowly flowing argon at ambient pressure (950 mbar). The emerging positively-chirped pulses are spectrally and temporally broadened, due to self-phase modulation in filamentation during propagation in the nonlinear medium. Subsequent reflection by chirped mirrors compensated the temporal broadening and the resulting pulses were shortened down to $10-12$ fs. There are no measurable energy losses in the cell.

After the chirped-mirror compressor, the radiation is reduced in energy to $E=0.3 \mathrm{~mJ}$ to avoid multiple filamenation when it is refocused $(f=1 \mathrm{~m})$ into a second windowless cell with Ar at ambient pressure. Under these conditions, a supercontinuum spectrum is produced exhibiting a very broad pedestal extending to $250 \mathrm{~nm}$ (210 nm starting from $6 \mathrm{fs})$. The pulses exhibited very good pointing stability, no conical emission, spectral and energy stability and transverse homogeneity. Whereas the stability results from avoiding multiple filmentation, the absence of conical emission is unique for the few-cycle input pulses. In a first attempt at shortening, part of the UV spectrum around $282 \mathrm{~nm}$ was cut out and after propagation in air and optical elements was guided to a prism compressor; a duration of about $30 \mathrm{fs}$ was achieved, determined in a cross-correlation set-up (two-colour ionization) between the UV and the IR fundamental pulses. It appeared very promsing for implementing in pump-probe experiments as the pump radiation; indeed pulses cut out at several wavelengths between 280 and $320 \mathrm{~nm}$ were used to excite $\mathrm{Cr}(\mathrm{CO})_{6}$. To avoid pulse splitting and higher order dispersion in $\mathrm{Ar}$, air and optical elements the supercontinuum was generated in the next experiment under conditions where the filament was terminated by a pressure gradient and the beam thereafter propagated only under vacuum. The pulses cut out e.g. near $290 \mathrm{~nm}$ turned out to be very short, about $10 \mathrm{fs}$, that is, slightly shorter than the input 810-nm pulses. The short durations and the 
tunability of the frequency have indeed proven very useful for applications of the supercontinuum radiation in time-resolved spectroscopy.

The pulses from the third- and fifth-harmonic $(270$ and $162 \mathrm{~nm})$ were generated in a shorter $(18-\mathrm{mm})$ Ar cell by $810-\mathrm{nm} 12 \mathrm{fs}$ input pulses $(E=0.7 \mathrm{~mJ})$; the short durations, $10 \mathrm{fs}$ at $162 \mathrm{~nm}$ and $10 \mathrm{fs}$ at $270 \mathrm{~nm}$ (or $30 \mathrm{fs}$ for TH with a beam path in air) and the conversion efficiencies obtained $(0.001 \%$ and $0.1 \%$, respectively) allowed for their implementation as the pump radiation in the investigation of the ethylene $\left(\mathrm{C}_{2} \mathrm{H}_{4}\right)$ and cyclohexadiene $\left(\mathrm{C}_{6} \mathrm{H}_{8}\right)$ reactions, respectively. Cell lengh times argon pressure is small enough to avoid development of any phase mismatch. The pulse durations in the UV were determined, as in all the above cases, by the $\mathrm{Xe}^{+}$yield, which represented the cross-correlation involving ionization by one $\mathrm{UV}$ photon and several 810-nm photons; the Xe ionization energy is $12.15 \mathrm{eV}$, corresponding to 8 photons of the fundamental.

$\mathrm{C}_{2} \mathrm{H}_{4}$ (and $\mathrm{C}_{2} \mathrm{D}_{4}$ ) was excited at 162-nm and the dynamics of the isomerization was probed by multiphoton $810-\mathrm{nm}$ ionization. With the time resolution being now about 15 times better than in previous experiments, five time constants (lifetimes) and four oscillations could be resolved for both molecules; they provide information and details indicating the complete path of the reaction, from the Franck-Condon (FC) region of the $\pi \pi^{*}$ state down to the ground state $\mathrm{S}_{0}$. It can be described by three steps: (1) After excitation to the Franck-Condon (FC) region of the $\pi \pi^{*}$ state the molecule relaxes along the $\mathrm{CC}$ twist and stretch coordinates. (About $10 \%$ of the population is excited to the $\pi 3 \mathrm{~s}$ Rydberg state, which is depleted within $80 \mathrm{fs}$ (115 fs for $\mathrm{C}_{2} \mathrm{D}_{4}$ ) also to the $\pi \pi^{*}$ state. The signals from these two states were successfully disentangled.) It leaves the $\mathrm{FC}$ region within 21 fs ( 24 fs for $\left.\mathrm{C}_{2} \mathrm{D}_{4}\right)$, while three vibrations are observed on this location, namely: $\mathrm{CC}$ stretch $1100 \mathrm{~cm}^{-1}\left(890 \mathrm{~cm}^{-1}\right.$ for $\left.\mathrm{C}_{2} \mathrm{D}_{4}\right), \mathrm{CC}$ twist 630 $\mathrm{cm}^{-1}\left(510 \mathrm{~cm}^{-1}\right.$ for $\left.\mathrm{C}_{2} \mathrm{D}_{4}\right)$ and a combination vibration of the two, $1570 \mathrm{~cm}^{-1}\left(1260 \mathrm{~cm}^{-1}\right.$ for $\mathrm{C}_{2} \mathrm{D}_{4}$ ). (2) Already before reaching the perpendicular minimum (which in fact is a saddle point if other coordinates are taken into account besides $\mathrm{CC}$ torsion) at torsional angle $\varphi=90^{\circ}$ the molecule starts changing direction towards H-migration. Within 17 fs (21 for $\left.\mathrm{C}_{2} \mathrm{D}_{4}\right) \mathrm{C}_{2} \mathrm{H}_{4}$ finds the way to the conical intersection with the ground state $S_{0}$ and ethylene (with $\varphi=0^{\circ}$ or $\left.\varphi=180^{\circ}\right)$ or alternatively ethylidene $\left(\mathrm{CHCH}_{3}\right)$ are formed, with much excess vibrational energy. In this window a CC stretch vibration was also observed, with frequency $1260 \mathrm{~cm}^{-1}$ $\left(1190 \mathrm{~cm}^{-1}\right.$ for $\left.\mathrm{C}_{2} \mathrm{D}_{4}\right)$ (3) The formation of two hot ground-state products was concluded from the two observed lifetimes there, 0.6 ps and 5 ps (1.6 ps and 11 ps for $\left.\mathrm{C}_{2} \mathrm{D}_{4}\right)$. The shorter one 
is assigned to ethylidene from which $\mathrm{C}_{2} \mathrm{H}_{4}$ is formed or $\mathrm{H} / \mathrm{H}_{2}$ is eliminated; the longer time constant is assigned to hot ethylene, which rearranges to ethylidene or loses $\mathrm{H}_{\text {or }} \mathrm{H}_{2}$. From the simultaneous formation of $\mathrm{CHCH}_{3}$ and other observations it is inferred that the $\mathrm{S}_{1} / \mathrm{S}_{0}$ conical intersection (CI) (the branching region for the path) involves partial H-migartion and a structure with an $\mathrm{H}$ bridge; the $\mathrm{CI}$ geometry has been controversial among quantum chemists.

Cyclohexadiene was excited at $270 \mathrm{~nm}$ by the 10 -fs third-harmonic pulses. The previously suggested reaction path for its ring-opening was now confirmed with the much better timeresolution, in particular with the oscillations that could now be resolved and assigned to vibrations. They are stimulated by slopes on the potential. The vibrational coordinates thus point to the local direction of the driving force and the reaction coordinate. The oscillations involve (A) two symmetric (symmetry species $a$ in $C_{2}$ ) stretch vibrations $\left(\mathrm{C}-\mathrm{C} 950 \mathrm{~cm}^{-1}\right.$ and $\mathrm{C}=\mathrm{C}$ $\left.1430 \mathrm{~cm}^{-1}\right)$ in the $\mathrm{FC}$ region, (B) a symmetric (a) ring puckering vibration $\left(300 \mathrm{~cm}^{-1}\right)$ on leaving the $1 \mathrm{~B}$ state around the $2 \mathrm{~B} / 1 \mathrm{~A}$ conical intersection and $(\mathrm{C})$ another ring deformation vibration $\left(140 \mathrm{~cm}^{-1}\right.$, antisymmtric $\left.(b)\right)$ on leaving the $2 \mathrm{~A}$ state via a conical intersection with the ground state. The reaction path can be summarized as follows: (1) After excitation in the Franck-Condon region of the 1B state the molecule travels along the $\mathrm{FC}$ active coordinates (certainly a general rule), involving bond stretching of the $\pi$ system and double-bond torsion; the latter motion distorts and stretches the $\mathrm{CH}_{2}-\mathrm{CH}_{2}$ bond, so that Woodward-Hoffmann interactions are activated only later, after this preparation. $\mathrm{C}_{6} \mathrm{H}_{8}$ leaves the $\mathrm{FC}$ region of the $1 \mathrm{~B}$ state within $21 \mathrm{fs}$. (2) It subsequently relaxes on the $1 \mathrm{~B}$ potential and the ring-opening begins on this location. The molecule reaches the $1 \mathrm{~B} / 2 \mathrm{~A} \mathrm{CI}$ and passes around the lower cone (with symmetry breaking towards the antisymmetric $b$ coordinate of the $140 \mathrm{~cm}^{-1}$ vibration) to the 2A state within another 35 fs. (3) Within 80 fs the molecule finds the 2A/1A CI and crosses to the ground state. This CI also involves the above antisymmetric distortion. The potential before this is very flat and the wave-packet motion is ballistic there. The ring-opening is already far advanced near the $2 \mathrm{~A}$ minimum, but is only completed on the ground-state surface.

The steps of the $\mathrm{CO}$ elimination in the dissociation of $\mathrm{Cr}(\mathrm{CO})_{6}$ were studied here for the excitation wavelengths at 270, 318 and $345 \mathrm{~nm}$ (about $30 \mathrm{fs}$ ). (The initial dynamics were also compared with those following absorption at 162, 196, 224, 242 and $282 \mathrm{~nm}$ ) (1) Upon absorption of one 270-nm photon and excitation of the $\mathrm{T}_{1 \mathrm{u}}$ metal-to-ligand charge-transfer (MLCT) state the molecule relaxes by electron back-transfer to a dissociative ligand-field (LF) state via an avoided crossing along $\mathrm{Cr}-\mathrm{CO}$ stretching, within 12.5 fs. (2) Further travel- 
ling on the LF state and dissociation require $18 \mathrm{fs} ; \mathrm{Cr}(\mathrm{CO})_{5}$ is produced in its first excited state $\left(\mathrm{S}_{1}\right)$. (3) It relaxes to the ground state $\left(\mathrm{S}_{0}\right)$ within $40 \mathrm{fs}$, via a symmetry-induced conical intersection in $D_{3 \mathrm{~h}}$ geometry. (4) The lifetime (towards further dissociation) of $\mathrm{Cr}(\mathrm{CO})_{5}$ in $\mathrm{S}_{0}$ is $1 \mathrm{ps}$; a vibration connected to pseudorotation $\left(\mathrm{Cr}-\mathrm{CO}\right.$ bending, $95 \mathrm{~cm}^{-1}$, symmetry species $a_{1}$ in $C_{4 \mathrm{v}}$ ) was observed there. In all the above steps of the reaction a totally symmetric $\mathrm{Cr}-\mathrm{CO}$ stretch vibration, already excited in the $\mathrm{FC}$ region and maintained throughout the whole reaction, could additionally be resolved (varying from 360 to $450 \mathrm{~cm}^{-1}$ in the different locations). The lifetimes, the oscillations and the reaction path turned out to be similar on exciting at 318 and $345 \mathrm{~nm}$, pointing to a common dissociation mechanism following the initial excitation of an MLCT state: the molecules after excitation flow to the LF state in one step, which then leads to dissociation in a second step. The similarity of the paths (including the observation of pseudorotation, which would not exist in a triplet pentacarbonyl) implies that (in contrast to textbook knowledge and some recent literature) no triplet state is involved in the dynamics of the dissociation, neither in the form of intersystem crossing from an initially excited singlet state, nor by exciting directly a triplet state in the intact molecule.

These pump-probe measurements elucidate the dynamics of fundamental processes in gasphase molecules, providing detailed insight in the microscopic nature of the dynamics of these systems and of ultrafast chemical reactions in general. The interpretation profited much from quantum chemical work. On the other hand our data can be the basis for testing high-level theoretical methods and to stimulate their further development. 


\section{References}

1. Trushin, S.A., et al., Supercontinuum extending from $>1000$ to $250 \mathrm{~nm}$, generated by focusing 10 -fs pulses at 805 nm into Ar. Applied Physics B, 2005. 80: p. 399-403.

2. Kosma, K., et al., Characterization of the supercontinuum radiation generated by self-focusing of fewcycle 800-nm pulses in argon. Journal of Modern Optics, 2008: p. in press.

3. Trushin, S.A., et al., Widely tunable ultraviolet sub-30 fs pulses from supercontinuum for transient spectroscopy. Applied Physics B, 2006. 85: p. 1-5.

4. Trushin, S.A., et al., Sub-10-fs supercontinuum radiation generated by filamentation of few-cycle 800-nm pulses in argon. Optics Letters, 2007. 32: p. 2432-2434.

5. Kosma, K., et al., 11-femtosecond pulses in the vacuum UV from fifth-harmonic generation of a Tisapphire laser. Optics Letters, 2008. 33: p. 723-725.

6. Kosma, K., et al., Ultrafast dynamics and coherent oscillations in ethylene and ethylene-d4 excited at 162 nm. Journal of Physical Chemistry A, 2008: p. submitted.

7. Kosma, K., et al., Cyclohexadiene ring opening observed with 13 fs resolution: Coherent oscillations confirm the reaction path. Physical Chemistry Chemical Physics, 2008: p. submitted.

8. Trushin, S.A., et al., Wavelength-independent ultrafast dynamics and coherent oscillation of a metalcarbon stretch vibration in photodissociation of $\operatorname{Cr}(C O)_{6}$ in the region of 270-345 $\mathrm{nm}$. Chemical Physics, 2008. 347: p. 309-323.

9. Hauri, C.P., et al., Generation of intense, carrier-envelope phase-locked few-cycle laser pulses through filamentation. Applied Physics B, 2004. 79: p. 673-677.

10. Brabec, T. and F. Krausz, Intense few-cycle laser fields: Frontiers of nonlinear optics. Review of Modern Physics, 2000. 72: p. 545-591.

11. Saleh, B.E.A. and M.C. Teich, Fundamentals of Photonics. 2007, New York: Wiley.

12. Krausz, F., Photonics (lecture notes). 2007.

13. Nibbering, E.T.J., et al., Determination of the inertial contribution to the nonlinear refractive index of air, N2, and $\mathrm{O} 2$ by use of unfocused high-intensity femtosecond laser pulses. Journal of the Optical Society of America B, 1997. 14: p. 650-660.

14. Alfano, R.R., The supercontinuum laser source. 1989, New York: Springer.

15. Shen, Y.R., The principles of nonlinear optics. 2003, Hoboken: Wiley.

16. Siegman, A.E., Lasers. University Science Books. 1986, Mill Valley, CA: University Science Books. 
17. Aközbek, N., C.M. Bowden, and S.L. Chin, Propagation dynamics of ultra-short high-power laser pulses in air: supercontinuum generation and transverse ring formation. Journal of Modern Optics, 2002. 49: p. 475-486.

18. Nikogosyan, D.N., Properties of Optical and Laser-Related Materials: A Handbook. 1997, New York: Wiley.

19. Diels, J.C. and W. Rudolph, Ultrashort Laser Pulse Phenomena. second ed. 2006: Elsevier.

20. Fork, R.L., O.E. Martinez, and J.P. Gordon, Negative dispersion using pairs of prisms. Opt. Lett., 1984. 9: p. $150-152$.

21. Baum, P., et al., Real-time characterization and optimal phase control of tunable visible pulses with a flexible compressor. Applied Physics B, 2002. 74: p. S219-S224.

22. Kasparian, J., R. Sauerbrey, and S.L. Chin, The critical laser intensity of self-guided light filaments in air. Applied Physics B, 2000. 71: p. 877-879.

23. Chin, S.L., et al., The propagation of powerful femtosecond laser pulses in optical media: physics, applications and new challenges. Canadian Journal of Physics, 2005. 83: p. 863-905.

24. Bergé, L., et al., Ultrashort filaments of light in weakly ionized, optically transparent media. Reports on Progress in Physics, 2007. 70: p. 1633-1713.

25. Chin, S.L., et al., Filamentation and supercontinuum generation during the propagation of powerful ultrashort laser pulses in optical media (white light laser). Jounal of Nonlinear Optical Physics and Materials, 1999. 8: p. 121-146.

26. Delone, N.B. and V.P. Krainov, Fundamentals of nonlinear optics of atomic gases. 1988, New York: Wiley.

27. Reintjes, J.F., Nonlinear optical parametric processes in liquids and gases. 1984, Orlando: Academic Press.

28. Aközbek, N., et al., Extending the supercontinuum spectrum down to $200 \mathrm{~nm}$ with few-cycle pulses. New Journal of Physics, 2006. 8: p. 177/1-12.

29. Becker, A., et al., Intensity clamping and re-focusing of intense femtosecond laser pulses in nitrogen. Applied Physics B, 2001. 73: p. 287-290.

30. Kienberger, R., et al., Sub-femtosecond X-ray pulse generation and measurement. Applied Physics B, 2002. 74: p. S3-S9.

31. Nishioka, H. and K. Ueda, Super-broadband continuum generation with transient self-focusing of a terawatt laser pulse in rare gases. Applied Physics B, 2003. 77: p. 171-175.

32. Chin, S.L., et al., Transverse ring formation of a focused femtosecond laser pulse propagating in air. Optics Communications, 2001. 188: p. 181-186. 
33. Nishioka, H., et al., Ultrabroadband flat continuum in multichannel propagation of terawatt Ti:sapphire laser pulses. Optics Letters, 1995. 20: p. 2505-2507.

34. Fuß, W., W.E. Schmid, and S.A. Trushin, Time-resolved dissociative intense-laser field ionization for probing dynamics: Femtosecond photochemical ring opening of 1,3-cyclohexadiene. Journal of Chemical Physics, 2000. 112: p. 8347-8362.

35. Papalazarou, E., et al., Spectral phase distribution retrieval through coherent control of harmonic generation. Physical Review Letters, 2006. 96: p. 163901.

36. Sekikawa, T., et al., Pulse compression of a high-order harmonic by compensating the atomic dipole phase. Physical Review Letters, 1999. 83: p. 2564-.

37. Liu, W., et al., Intensity clamping of a femtosecond laser pulse in condensed matter. Optics Communications, 2002. 202: p. 189-197.

38. Théberge, F., et al., Ultrabroadband continuum generated in air (down to $230 \mathrm{~nm}$ ) using ultrashort and intense laser pulses. Applied Physics B, 2005. 80: p. 221-225.

39. Manassah, J.T., Simple models of self-phase and induced phase modulation, in The supercontinuum laser source, R.R. Alfano, Editor. 1989, Springer: New York. p. 184-294.

40. Koprinkov, I.P., et al., Self-compression of high-intensity femtosecond optical pulses and spatio-temporal soliton generation. Physical Review Letters, 2000. 84: p. 3847-3850.

41. Gaeta, A.L., Catastrophic collapse of ultrashort pulses. Physical Review Letters, 2000. 84: p. 3582-3585.

42. Méchain, G., et al., Long-range self-channeling of infrared laser pulses in air: a new propagation regime without ionization. Applied Physics B, 2004. 79: p. 379-382.

43. Couairon, A., Filament length of powerful laser pulses. Applied Physics B, 2003. 76: p. 789-792.

44. Kandidov, V.P., I.S. Golubtsov, and O.G. Kosareva, Supercontinuum sources in a high-power femtosecond laser pulse propagating in liquids and gases. Quantum Electronics, 2004. 34: p. 348-354.

45. Nibbering, E.T.J., et al., Conical emission from self-guided femtosecond laser pulses in air. Optics Letters, 1996. 21: p. 62-64.

46. Liu, W., et al., The influence of divergence on the filament length during the propagation of intense ultrashort laser pulses. Applied Physics B, 2006. 82: p. 373-376.

47. Aumiler, D., T. Ban, and G. Pichler, Femtosecond laser-induced cone emission in dense cesium vapor. Physical Review A, 2005. 71: p. 063803/1-6.

48. Couairon, A. and A. Mysyrowicz, Femtosecond filamentation in transparent media. Physics Reports, 2007. 441: p. 47-189. 
49. Oberthaler, M. and R.A. Höpfl, Special narrowing of ultrashort laser pulses by self-phase modulation in optical fibers. Applied Physics Letters, 1993. 63: p. 1017-1019.

50. Couairon, A., et al., Self-compression of ultra-short laser pulses down to one optical cycle by filamentation. Journal of Modern Optics, 2006. 53: p. 75-85.

51. Stibenz, G., N. Zhavoronkov, and G. Steinmeyer, Self-compression of millijoule pulses to 7.8 fs duration in a white-light filament. Optics Letters, 2006. 31: p. 274-276.

52. Skupin, S., et al., Self-compression by femtosecond pulse filamentation: Experiments versus numerical simulations. Physical Review E, 2006. 74: p. 056604/1-9.

53. Aközbek, N., et al., Third-harmonic generation and self-channeling in air using high-power femtosecond laser pulses. Physical Review Letters, 2002. 89: p. 143901/1-4.

54. Zheltikov, A.M., Third-harmonic generation with no signal at 3w. Physical Review A, 2005. 72: p. $043812 / 1-4$

55. Tzankov, P., et al., High-power fifth harmonic generation of femtosecond pulses in the vacuum ultraviolet using a Ti:sapphire laser. Optics Express, 2007. 15: p. 6389-6395.

56. Jailaubekov, A.E. and S.E. Bradforth, Tunable 30-femtosecond pulses across the deep ultraviolet. Applied Physics Letters, 2005. 87: p. 021107/1-3.

57. Zewail, A.H., Laser femtochemistry. Science, 1988. 242: p. 1645.

58. Fuß, W., et al., The photochemical cis-trans isomerization of free stilbene molecules follows a Hula-twist pathway. Angewandte Chemie International Edition in English, 2004. 43: p. 4178-4182.

59. Fuß, W., et al., Competing ultrafast cis-trans isomerisation and ring closure of cyclohepta-1,3-diene and cyclo-octa-1,3-diene. Molecular Physics, 2006. 104: p. 1133-1143.

60. Fuß, W., W.E. Schmid, and S.A. Trushin, Nonresonant dissociative photoionization for monitoring ultrafast dynamics of photochemical reactions. Indian Society for Radiation and Photochemical Sciences Bulletin, 2000. 11: p. 7-17.

61. Born, M. and R. Oppenheimer, Annalen der Physik, 1927. 84: p. 457.

62. Levine, I.N., Quantum chemistry. 5 ed. 2000, Upper Saddle River, New Jersey: Prentice-Hall.

63. Levine, I.N., Molecular spectroscopy. 1975, New York: Wiley.

64. Worth, G.A. and L.S. Cederbaum, Ann. Rev. Phys. Chem., 2004. 55: p. 127.

65. Herzberg, G., Molecular Spectra and Molecular Structure, Vol. 3: Electronic Spectra and Electronic Structure of Polyatomic Molecules. Vol. 3. 1966, New York: Van Nostrand Reinhold.

66. Manthe, U. and H. Köppel, Dynamics on potential energy surfaces with a conical intersection: Adiabatic, intermediate, and diabatic behavior. Journal of Chemical Physics, 1990. 93: p. 1658-1669. 
67. Domcke, W., D.R. Yarkony, and H. Köppel, Conical intersections. 2004, London: World Scientific.

68. Klessinger, M. and J. Michl, Excited states and photochemistry of organic molecules. 1995, New York: $\mathrm{VCH}$.

69. Paterson, M.J., et al., Phys. Chem. Chem. Phys., 2005. 7: p. 2100.

70. Jahn, H. and E. Teller, Proc. Roy. Soc. A, 1937. 161: p. 220.

71. Franck, J., Trans. Faraday Soc., 1926. 21: p. 536.

72. Condon, E.U., Physical Review, 1928. 32: p. 858.

73. Hudson, B.S. and B.E. Kohler, Linear polyene electronic structure and spectroscopy. Annual Review of Physical Chemistry, 1974. 25: p. 437-460.

74. Fuß, W., W.E. Schmid, and S.A. Trushin, Ultrafast electronic relaxation of s-trans butadiene. Chemical Physics Letters, 2001. 342: p. 91-98.

75. Fuß, W., et al., Spectroscopy and ultrafast dynamics of the $2 A_{1}$ state of Z-hexatriene in gas phase. Journal of Chemical Physics, 1997. 106: p. 2205-2211.

76. Fuß, W., et al., Pathway approach to ultrafast photochemistry: potential surfaces, conical intersections and isomerizations of small polyenes. Chemical Physics, 1998. 232: p. 161-174.

77. Pedersen, S. and A.H. Zewail, Femtosecond real-time probing of reactions. XXII. Kinetic description of probe absorption, fluorescence, depletion and mass spectrometry. Molecular Physics, 1996. 89: p. 14551502.

78. Trushin, S.A., et al., Coherent oscillations in the charge-transfer system 4-dimethylamino-benzonitrile. Chemical Physics Letters, 2003. 376: p. 282-291.

79. Fuß, W., et al., Ultrafast relaxation and coherent oscillations in aminobenzonitrile derivatives in the gas phase probed by intense-field ionization. Physical Chemistry Chemical Physics, 2007. 9: p. 1151-1169.

80. Trushin, S.A., W. Fuß, and W.E. Schmid, Dissociative ionization at high laser intensities: Importance of resonances and relaxation for fragmentation. Journal of Physics B: Atomic and Molecular Optical Physics, 2004. 37: p. 3987-4011.

81. Farmanara, P., et al., Ultrafast internal conversion and photodissociation of molecules excited by femtosecond 155 nm pulses. Journal of Chemical Physics, 1999. 111: p. 6264-6270.

82. Farmanara, P., V. Stert, and W. Radloff, Ultrafast internal conversion and fragmentation in electronically excited $\mathrm{C}_{2} \mathrm{H}_{4}$ and $\mathrm{C}_{2} \mathrm{H}_{3} \mathrm{Cl}$ molecules. Chemical Physics Letters, 1998. 288: p. 518-522.

83. Mestdagh, J.M., et al., Prereactive evolution of monoalkenes excited in the $6 \mathrm{eV}$ region. Journal of Chemical Physics, 2000. 113: p. 237-248. 
84. Stert, V., et al., Femtosecond time-resolved dynamics of the electronically excited ethylene molecule. Chemical Physics Letters, 2004. 388: p. 144-149.

85. Merer, A.J. and R.S. Mulliken, Ultraviolet spectra and excited states of ethylene and its alkyl derivates. Chemical Reviews, 1969. 69: p. 639-656.

86. Robin, M.B., Higher excited states of polyatomic molecules. Vol. 3. 1985, New York: Academic Press. 213-227.

87. Foo, P.D. and K.K. Innes, New experimental tests of existing interpretations of electronic transitions in ethylene. Journal of Chemical Physics, 1974. 60: p. 4582-4589.

88. Sension, R.J. and B.S. Hudson, Vacuum ultraviolet resonance Raman studies of the excited electronic states of ethylene. Journal of Chemical Physics, 1989. 90: p. 1377-1389.

89. Ziegler, L.D. and B.S. Hudson, Resonance Raman scattering of ethylene: Evidence for a twisted geometry in the V state. Journal of Chemical Physics, 1983. 79: p. 1197-1202.

90. Wilkinson, P.G. and R.S. Mulliken, Far ultraviolet absorption spectra of ethylene and ethylene-d4. Journal of Chemical Physics, 1955. 23: p. 1895-1907.

91. Orkin, V.L., R.E. Huie, and M.J. Kurylo, Rate constants for the reactions of OH with HFC-245cb $\left(\mathrm{CH}_{3} \mathrm{CF}_{2} \mathrm{CF}_{3}\right)$ and some fluoroalkenes $\left(\mathrm{CH}_{2} \mathrm{CHCF}_{3}, \mathrm{CH}_{2} \mathrm{CFCF}_{3}, \mathrm{CF}_{2} \mathrm{CFCF}_{3}\right.$, and $\left.\mathrm{C}_{2} \mathrm{~F}_{4}\right)$. Journal of Physical Chemistry A, 1997. 101: p. 9118-9124.

92. Barbatti, M., J. Paier, and H. Lischka, Photochemistry of ethylene: A multireference configuration interaction investigation of the excited-state surfaces. Journal of Chemical Physics, 2004. 121: p. 11614-11624.

93. Krawczyk, R.P., et al., Photoinduced dynamics of the valence states of ethene: a six-dimensional potential-energy surface of three electronic states with several conical intersections. Journal of Chemical Physics, 2003. 119: p. 1397-1411.

94. Rijkenberg, R.A. and W.J. Buma, High-resolution excited-state photoelectron spectroscopy of the lower Rydberg states of jet-cooled C2H4 and C2D4. Journal of Physical Chemistry A, 2002. 106: p. 3727-3737.

95. Merer, A.J. and L. Schoonveld, Electronic spectrum of ethylene. I. The $1744 \AA$ Rydberg transition. Canadian Journal of Physics, 1969. 47: p. 1731-1743.

96. Willitsch, S., U. Hollenstein, and F. Merkt, Ionization from a double bond: Rovibronic photoionization dynamics of ethylene, large amplitude torsional motion and vibronic coupling in the ground state of C2H4+. Journal of Chemical Physics, 2004. 120: p. 1761-1774.

97. Ben-Nun, M. and T.J. Martínez, Photodynamics of ethylene: ab initio studies of conical intersections. Chemical Physics, 2000. 259: p. 237-248.

98. Ben-Nun, M. and T.J. Martínez, Ab initio quantum molecular dynamics. Advances in Chemical Physics, 2002. 121: p. 439-452. 
99. Freund, L. and M. Klessinger, Photochemical reaction pathways of ethylene. International Journal of Quantum Chemistry, 1998. 70: p. 1023-1028.

100. Viel, A., et al., Photoinduced dynamics of ethene in the $N, V$, and $Z$ valence states: A six-dimensional nonadiabatic quantum dynamics investigation. Journal of Chemical Physics, 2004. 120: p. 11000-11010.

101. Steinmetz, M.G., Photochemistry with short UV light, in Organic Photochemistry, A. Padwa, Editor. 1987, Marcel Dekker: New York. p. 67-158.

102. Collin, G.J., Photochemistry of simple olefins: chemistry of electronic excited states or hot ground state? Advances in Photochemistry, 1988. 14: p. 135-175.

103. Lee, S.H. and Y.T. Lee, Dependence of the distributions of kinetic energies of products on photoionization energy in the photodissociation of ethene at $157 \mathrm{~nm}$. Chemical Physics Letters, 2006. 419: p. 158-163.

104. Shimanouchi, T., Tables of molecular frequencies consolidated. Vol. 1. 1972: NSRDS-NBS 39. 139.

105. Brill, M.R., et al., Photoinduced nonadiabatic dynamics of ethene: Six-dimensional wave packet propagations using two different approximations of the kinetic energy operator. Chemical Physics, 2007. 338: p. 186-199.

106. Barbatti, M., M. Ruckenbauer, and H. Lischka, The photodynamics of ethylene: A surface-hopping study on structural aspects. Journal of Chemical Physics, 2005. 122: p. 174307/1-9.

107. Molina, V., et al., On the low-lying singlet excited states of styrene: A theoretical contribution. Physical Chemistry Chemical Physics, 2000. 2: p. 2211-2217.

108. McDiarmid, R. and E. Charney, Far-ultraviolet spectrum of ethylene and ethylene-d4. Journal of Chemical Physics, 1967. 47: p. 1517-1524.

109. Trushin, S.A., et al., Coherent oscillation and ultrafast internal conversion of tetrafluoroethylene after excitation at 197 nm. Chemical Physics - Physical Chemistry, 2004. 5: p. 1389-1397.

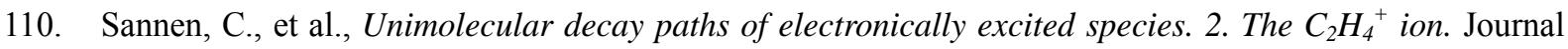
of Chemical Physics, 1981. 74: p. 2402-2411.

111. Oblinger, M., A.J. Lorquet, and J.C. Lorquet, $\mathrm{Ab}$ initio study of the reaction $\mathrm{C}_{2} \mathrm{H}_{4}{ }^{+}->\mathrm{C}_{2} \mathrm{H}_{3}{ }^{+}+\mathrm{H}$. International Journal of Mass Spectrometry and Ion Processes, 1997. 167-168: p. 149-160.

112. Chang, A.H.H., et al., Ab initio/RRKM approach toward the understanding of ethylene photodissociation. Journal of Chemical Physics, 1998. 109: p. 2748-2761.

113. Modarelli, D.A. and M.S. Platz, Experimental evidence for ethylidene-d4. Journal of the American Chemical Society, 1993. 115: p. 470-475.

114. Raghavachari, K., M.J. Frisch, and J. Pople, The ground-state singlet potential surface for $\mathrm{C}_{2} \mathrm{H}_{4}$. Chemical Physics Letters, 1982. 85: p. 145-149. 
115. Evanseck, J.D. and K.N. Houk, Stereoselectivity of hydrogen 1,2-shifts in singlet alkylcarbenes: A quantitative theoretical assessment of ground-state orbital alignment and torsional and steric effects. Journal of the American Chemical Society, 1990. 112: p. 9148-9156.

116. Peña-Gallego, A., E. Martínez-Nuñez, and S.A. Vázquez, Dissociation of ethylene and several deuterated derivatives at 193 and $157 \mathrm{~nm}$ by direct classical trajectories. Chemical Physics Letters, 2002. 353: p. 418-425.

117. Costa, F., Electronic structure study of the reaction $\mathrm{C}_{2} \mathrm{H}_{4}{ }^{+}->\mathrm{C}_{2} \mathrm{H}_{2}{ }^{+}+\mathrm{H}_{2}$. International Journal of Quantum Chemistry, 2006. 106: p. 2763-2771.

118. Kim, M.H., et al., Vibrationally mediated photodissociation of $\mathrm{C}_{2} \mathrm{H}_{4}{ }^{+}$. Journal of Physical Chemistry A, 2007. 111: p. 7472-7480.

119. Stockbauer, R. and M.G. Inghram, Threshold photoelectron -photoion coincidence mass spectrometric study of ethylene and ethylene-d4. Journal of Chemical Physics, 1975. 62: p. 4862-4870.

120. Kimura, K., et al., Handbook of HeI photoelectron spectra of fundamental organic molecules. 1981, Tokyo: Japan Scientific Soc. Press.

121. Wilsey, S. and K.N. Houk, "H/vinyl" and "alkyl/vinyl" conical intersections leading to carbene formation from the excited states of cyclohexene and norbornene. Journal of the American Chemical Society, 2002. 124: p. 11182-11190.

122. Ben-Nun, M. and T.J. Martínez, Ab initio molecular dynamics study of cis-trans isomerization in ethylene. Chemical Physics Letters, 1998. 298: p. 57-65.

123. Ben-Nun, M., J. Quenneville, and T.J. Martínez, Ab initio multiple spawning: Photochemistry from first principles quantum molecular dynamics. Journal of Physical Chemistry A, 2000. 104: p. 5161-5175.

124. Quenneville, J., M. Ben-Nun, and T.J. Martínez, Photochemistry from first principles - advances and future prospects. Journal of Photochemistry and Photobiology A: Chemistry, 2001. 144: p. 229-235.

125. Laino, T. and D. Passerone, Pseudo-dynamics and band optimizations: Shedding light into conical intersection seams. Chemical Physics Letters, 2004. 389: p. 1-6.

126. Robin, M.B., Higher excited states of polyatomic molecules. Vol. 2. 1975, New York: Academic Press. 166-181.

127. Miron, E., B. Raz, and J. Jortner, Intravalence and extravalence excitations of the ethylene molecule in liquid and solid rare gas matrices. Journal of Chemical Physics, 1972. 56: p. 5265-5272.

128. Dauber, P., et al., The vibronic structure of crystalline ethylene. Chemical Physics, 1975. 7: p. 108-115.

129. Merer, A.J. and J.K.G. Watson, Symmetry considerations for internal rotation in ethylene-like molecules. Journal of Molecular Spectroscopy, 1973. 47: p. 499-514. 
130. Borelli, R. and A. Peluso, The vibrational progressions of the $N->V$ electronic transition of ethylene: A test case for the computation of Franck-Condon factors of highly flexible photoexcited molecules. Journal of Chemical Physics, 2006. 125: p. 194308/1-8.

131. Hazra, A., H.H. Chang, and M. Nooijen, First principles simulation of the UV absorption spectrum of ethylene using the vertical Franck-Condon approach. Journal of Chemical Physics, 2004. 121: p. 2125 2136.

132. Warshel, A.M. and M. Karplus, Vibrational structure of electronic transitions in conjugated molecules. Chem. Phys. Lett., 1972. 17: p. 7-14.

133. Mebel, A.M., Y.T. Chen, and S.H. Lin, pi-pi* vibronic spectrum of ethylene from ab initio calculations of the Franck-Condon factors. Chemical Physics Letters, 1996. 258: p. 53-62.

134. Mebel, A.M., et al., Ab initio calcualtions of vibronic spectra and dynamics for small polyatomic molecules: Role of the Duschinsky effect. Journal of Physical Chemistry A, 1999. 103: p. 10674-10690.

135. Petrongolo, C., R.J. Buenker, and S.D. Peyerimhoff, Nonadiabatic treatment of the intensity distribution in the $V-N$ bands of ethylene. Journal of Chemical Physics, 1982. 76: p. 3655-3667.

136. Petrongolo, C., R.J. Buenker, and S.D. Peyerimhoff, Nonadiabatic investigation of the V-N spectrum of ethylene in a new diabatic representation. Journal of Chemical Physics, 1983. 78: p. 7284-7289.

137. Williams, B.A. and T.A. Cool, Two-photon spectroscopy of Rydberg states of jet-cooled $\mathrm{C}_{2} \mathrm{H}_{4}$ and $\mathrm{C}_{2} \mathrm{D}_{4}$. Journal of Chemical Physics, 1991. 94: p. 6358-6366.

138. Barbatti, M., et al., Semiempirical molecular dynamics investigation of the excited state lifetime of ethylene. Chemical Physics Letters, 2005. 401: p. 276-281.

139. Mebel, A.M., M. Hayashi, and S.H. Lin, Ab initio calcualtions of vibronic coupling. Application to symmetry-forbidden vibronic spectra and internal conversion in ethylene. Chemical Physics Letters, 1997. 274: p. 281-292.

140. Hayashi, M., et al., Ab initio calculations of radiationless transitions between excited and ground singlet electronic states of ethylene. Journal of Chemical Physics, 1998. 108: p. 2044-2055.

141. Granucci, G., M. Persico, and A. Toniolo, Direct semiclassical simulation of photochemical processes with semiempirical wave functions. Journal of Chemical Physics, 2001. 114: p. 10608-10615.

142. Viel, A., et al., The sudden-polarization effect and its role in the ultrafast photochemistry of ethene. Angewandte Chemie International Edition in English, 2003. 42: p. 3434-3436.

143. Evleth, E.M. and A. Sevin, A theoretical study of the role of valence and Rydberg states in the photochemistry of ethylene. Journal of the American Chemical Society, 1981. 103: p. 7414-7422.

144. Ohmine, I., Mechanisms of nonadiabatic transitions in photoisomerization processes of conjugated molecules: Role of hydrogen migrations. Journal of Chemical Physics, 1985. 83: p. 2348-2362. 
145. Reid, P.J., et al., Determination of pericyclic photochemical reaction dynamics with resonance Raman spectroscopy. Journal of Physical Chemistry, 1994. 98: p. 5597-5606.

146. Carreira, L.A., R.O. Carter, and J.R. Durig, Raman spectra of gases. VII. Barriers to planarity in 1,4- and 1,3-cyclohexadiene. Journal of Chemical Physics, 1973. 59: p. 812-816.

147. Saebø, S. and J.E. Boggs, Structure and conformations of cyclohexene,1,4-cyclohexadiene, and 1,3cyclohexadiene. Journal of Molecular Structure, 1981. 73: p. 137-144.

148. Sygula, A. and P.W. Rabideau, An ab initio study of the structures and the inversion barriers in 1,3cyclohexadiene, 1,2-dihydronaphthalene and 9,10-dihydrophenanthlene. Journal of Molecular Structure (Theochem), 1992. 262: p. 117-130.

149. Autrey, D., J. Choo, and J. Laane, Spectroscopic determination of the ring-twisting potential energy function of 1,3-cyclohexadiene and comparison with ab initio calculations. Journal of Physical Chemistry A, 2001. 105: p. 10230-10236.

150. Woodward, R.B. and R. Hoffmann, The Conservation of Orbital Symmetry. 1970, Weinheim: VCH.

151. Garavelli, M., et al., Reaction path of a sub-200 femtosecond photochemical electrocyclic reaction. Journal of Physical Chemistry A, 2001. 105: p. 4458-4469.

152. van der Lugt, W.T.A.M. and L.J. Oosterhoff, Symmetry control and photoinduced reactions. Journal of the American Chemical Society, 1969. 91: p. 6042-6049.

153. Celani, P., et al., What happens during the picosecond lifetime of $2 A_{1}$ cyclohexa-1,3-diene? A CAS-SCF study of the cyclohexadiene/hexatriene photochemical conversion. Journal of the American Chemical Society, 1994. 116: p. 10141-10151.

154. Kuthirummal, N., et al., Spectroscopy and femtosecond dynamics of the ring opening reaction of 1,3cyclohexadiene. Journal of Chemical Physics, 2006. 125: p. 133307/1-8.

155. Tamura, H., et al., Ab initio nonadiabatic quantum dynamics of cyclohexadiene/hexatriene ultrafast photoisomerization. Journal of Chemical Physics, 2006. 124: p. 084313/1-13.

156. Trushin, S.A., et al., Femtosecond photochemical ring-opening of 1,3-cyclohexadiene studied by timeresolved intense-field ionization. Journal of Chemical Physics, 1997. 106: p. 9386-9389.

157. Trulson, M.O., G.D. Dollinger, and R.A. Mathies, Excited state structure and femtosecond ring-opening dynamics of 1,3-cyclohexadiene from absolute resonance Raman intensities. Journal of Chemical Physics, 1989. 90: p. 4274-4281.

158. Lawless, M.K., S.D. Wickham, and R.A. Mathies, Resonance Raman view of pericyclic photochemical ring-opening reactions: Beyond the Woodward-Hoffmann rules. Accounts of Chemical Research, 1995. 28: p. 493-502.

159. Thorne, A.P., U. Litzén, and S. Johansson, Spectrophysics. 1999, Heidelberg: Springer. 
160. Di Lauro, C. and N. Neto, Vibrational spectrum and normal-mode ananlysis of 1,3-cyclohexadiene. Journal of Molecular Structure, 1972. 3: p. 219-226.

161. Trulson, M.O., G.D. Dollinger, and R.A. Mathies, Femtosecond photochemical ring-opening dynamics of 1,3-cyclohexadiene from resonance Raman intensities. J. Am. Chem. Soc., 1987. 109: p. 586-587.

162. de Vivie-Riedle, R., personal communication. 2007.

163. Carroll, E.C., et al., Spectral phase effects on nonlinear resonant photochemistry of 1,3-cyclohexadiene in solution. Journal of Chemical Physics, 2006. 124: p. 114506/1-10.

164. Trushin, S.A., et al., Femtosecond dynamics of hydrogen migration and internal conversion in cycloheptatriene and derivatives studied by intense-field dissociative ionization. Physical Chemistry Chemical Physics, 1999. 1: p. 1431-1440.

165. Fuß, W., et al., Forward and backward pericyclic photochemical reactions have intermediates in common. ChemPhysChem, 2007. 8: p. 592-598.

166. Geppert, D. and R. de Vivie-Riedle, Reaction velocity control by manipulating the momentum of a nuclear wavepacket with phase-sensitive optimal control theory. Chemical Physics Letters, 2005. 404: p. 289-295.

167. Boggio-Pasqua, M., et al., Can diarylethene photochromism be explained by a reaction path alone? A CASSCF study with model MMVB dynamics. Journal of Physical Chemistry A, 2003. 107: p. 1113911152.

168. Anderson, N.A., J.J. Shiang, and R.J. Sension, Subpicosecond ring opening of 7-dehydrocholesterol studied by ultrafast spectroscopy. Journal of Physical Chemistry A, 1999. 103: p. 10730-10736.

169. Heinz, B., et al., Comparing a photoinduced pericyclic ring opening and closure: Differences in the excited state pathways. Journal of the American Chemical Society, 2007. 129: p. 8577-8584.

170. Torii, H. and M. Tasumi, Re-examination of the vibrational force fields of trans- and cis-1,3,5-hexatriene: Effect of electron correlation, transferability of scale factors and correspondence of normal modes between the two isomers. Vibrational Spectroscopy, 1995. 8: p. 205-214.

171. van der Lugt, W.T.A.M. and L.J. Oosterhoff, Quantum-chemical interpretation of photo-induced electrocyclic reactions. Chemical Communications, 1968: p. 1235-1236.

172. Dou, Y., S. Yuan, and G.V. Lo, Ring opening reaction of 1,3-cyclohexadiene studied by semiclassical dynamics simulation. Applied Surface Science, 2007. 253: p. 6494-6408.

173. Nonnenberg, C., S. Grimm, and I. Frank, Restricted open-shell Kohn-Sham theory of pi-pi* transitions. 2. Simulation of photochemical reactions. Journal of Chemical Physics, 2003. 119: p. 11585-11590. 
174. Bernardi, F., M. Olivucci, and M.A. Robb, The role of conical intersections and excited state reaction paths in photochemical pericyclic reactions. Journal of Photochemistry and Photobiology A: Chemistry, 1997. 105: p. 365-371.

175. Fuß, W. Branching and momentum effects in photochemistry. in International Conference on Computational Methods in Science and Engineering. 2007. Corfu (Greece): Amerian Institute of Physics.

176. Celani, P., et al., Do photochemical ring-openings occur in the spectroscopic state? $1 B_{2}$ pathways for the cyclohexadiene/hexatriene photochemical interconversion. Journal of Physical Chemistry, 1996. 100: p. 19364-19366.

177. Garavelli, M., et al., Potential energy surfaces for ultrafast photochemistry: Static and dynamic aspects. Faraday Discussions, 1998. 110: p. 51-70.

178. Beach, N.A. and H.B. Gray, Electronic structures of metal hexacarbonyls. Journal of the American Chemical Society, 1968. 90: p. 5713-5721.

179. Rosa, A., et al., Electronic spectra of $M(C O)_{6}(M=C r, M o, W)$ revisited by a relativistic TDDFT approach. Journal of the American Chemical Society, 1999. 121: p. 10356-1065.

180. Baerends, E.J. and A. Rosa, Metal-CO photodissociation in transition metal complexes: the role of ligand-field and charge-transfer states in the photochemical dissociation of metal-ligand bonds. Coordination Chemistry Reviews, 1998. 177: p. 97-125.

181. Crabtree, R.H., The organometallic chemistry of the transition metals. 1988, New York: Wiley.

182. Jordan, R.B., Reaction mechanisms of inorganic and organometallic systems. 1991, Oxford: Oxford Univ. Press.

183. Lees, A.J., Luminescence properties of organometallic complexes. Chemical Reviews, 1987. 87: p. 711743.

184. Pierloot, K., E. Tsokos, and L.G. Vanquickenborne, Optical spectra of $\mathrm{Ni}(\mathrm{CO})_{4}$ and $\mathrm{Cr}(\mathrm{CO})_{6}$ revisited. Journal of Physical Chemistry, 1996. 100: p. 16545-16550.

185. Pollak, C., A. Rosa, and E.J. Baerends, Cr-CO photodissociation in $\mathrm{Cr}(\mathrm{CO})_{6}$ : Reassessment of the role of ligand-field excited states in the photochemical dissociation of metal-ligand bonds. Journal of the American Chemical Society, 1997. 119: p. 7324-7329.

186. Hummel, P., et al., Ligand-field excited states of metal hexacarbonyls. Inorganic Chemistry, 2005. 44: p. 2454-2458.

187. Ben Amor, N., et al., The electronic spectroscopy of transition metal carbonyls: The tough case of $\operatorname{Cr}(\mathrm{CO})_{6}$. Chemical Physics Letters, 2006. 421: p. 378-382.

188. Paterson, M.J., et al., Non-adiabatic direct dynamics study of chromium hexacarbonyl photodissociation. Journal of Physical Chemistry A, 2002. 106: p. 10494-10504. 
189. Trushin, S.A., W. Fuß, and W.E. Schmid, Conical Intersections, pseudorotation and coherent oscillations in ultrafast photodissociation of group-6 metal hexacarbonyls. Chemical Physics, 2000. 259: p. 313-330.

190. Trushin, S.A., et al., Femtosecond dynamics and vibrational coherence in gas phase UV photodecomposition of $\operatorname{Cr}(\mathrm{CO})_{6}$. Journal of Physical Chemistry A, 1998. 102: p. 4129-4137.

191. Geoffroy, G.L. and M.S. Wrighton, Organometallic Photochemistry. 1979, New York: Academic Press.

192. Worth, G., G. Welch, and M.J. Paterson, Wavepacket dynamics staudy of $\operatorname{Cr}(\mathrm{CO})_{5}$ after formation by photodissociation: relaxation through an $(E+A) x e$ Jahn-Teller conical intersection. Molecular Physics, 2006. 104: p. 1095-1105.

193. Rayner, D.M., et al., Branching ratios and bond dissociation energies from the excimer laser photolysis of group 6 metal carbonyls. Journal of Chemical Physics, 1991. 94: p. 5471-5480.

194. Simon, J.D. and K.S. Peters, Chemical Physics Letters, 1983. 98: p. 53.

195. Rothberg, L.J., et al., Journal of the American Chemical Society, 1982. 104: p. 3536.

196. Welch, J.A., K.S. Peters, and V. Vaida, Journal of Physical Chemistry, 1982. 86: p. 1941.

197. Jonas, V. and W. Thiel, Theoretical study of the vibrational spectra of the transition metal carbonyls $M(C O)_{6}(M=C r, M o, W), M(C O)_{5}(M=F e, R u, O s)$, and $M(C O)_{4}(M=N i, P d, P t)$. Journal of Chemical Physics, 1995. 102: p. 8474-8483.

198. Rocha, A.B., Intensity of d-d symmetry-forbidden electronic transition in $\operatorname{Cr}(\mathrm{CO})_{6}$. Journal of Physical Chemistry A, 2007. 111: p. 4711-4713.

199. Adelman, D. and D.P. Gerrity, UV resonance Raman studies of group 6 transition-metal carbonyls: Evidence of Jahn-Teller distortion in excited states of the lowest allowed charge-transfer transitions. Journal of Physical Chemistry, 1990. 94: p. 4055-4060.

200. Yu, S.-C., S.-B. Ko, and J.B. Hopkins, Investigation of the reaction coordinate for dissociation in Cr(CO) $)_{6}$ using resonance Raman spectroscopy. Bull. Korean Chem. Soc., 1995. 16: p. 1043-1045.

201. Ko, S.-B., S.-c. Yu, and J.B. Hopkins, Ultrafast investigation of vibrational relaxation and solvent coordination following photodissociation of $\operatorname{Cr}(C O)_{6}$. Bull. Korean Chem. Soc., 1994. 15: p. 762-765.

202. Fletcher, T.R. and R.N. Rosenfeld, Studies on the photochemistry of chromium hexacarbonyl in the gas phase: primary and secondary processes. Journal of the American Chemical Society, 1985. 107: p. 22032212.

203. Holland, J.P. and R.N. Rosenfeld, Energy disposal in the photofragmentation of $W(C O)_{6}$ : Experimental observations and physical models. Journal of Chemical Physics, 1988. 89: p. 7217-7225.

204. Holland, J.P. and R.N. Rosenfeld, A time-resolved tunable diode laser study of the photodissociation dynamics of $W(C O)_{6}$. Chemical Physics Letters, 1988. 145: p. 481-485. 
205. Trogler, W.C., S.E. Desjardin, and E.I. Solomon, Spectroscopic and theoretical analysis of the intense $1 T_{1 u}<-1 A_{1 g}$ transitions in $\mathrm{Mo}(\mathrm{CO})_{6}$ and $\mathrm{W}(\mathrm{CO})_{6}$. Inorganic Chemistry, 1979. 18: p. 2131-2136.

206. Dantus, M., et al., Femtosecond real-time probing of reactions. V. The reaction of IHgI. Journal of Chemical Physics, 1989. 91: p. 7437-7450.

207. Gruebele, M., G. Roberts, and A.H. Zewail, Femtochemistry of the reaction of IHgI: theory versus experiment. Philosophical Transactions of the Royal Society London A, 1990. 332: p. 223-243.

208. Sorgues, S., et al., Wave packet movements near the conical intersection between two excited potential surfaces may create observable molecular oscillations. Physical Review Letters, 2003. 91: p. 103001 1-4.

209. Nasielski, J. and A. Colas, The primary process in the photochemistry of group 6B metal hexacarbonyls. 2. Inorganic Chemistry, 1978. 17: p. 237.

210. Joly, A.G. and K.A. Nelson, Metal carbonyl photochemistry in organic solvents: femtosecond transient absorption and preliminary resonance Raman spectroscopy. Chemical Physics, 1991. 152: p. 69-82. 


\section{Acknowledgements}

I am very thankful to Professor Ferenc Krausz for the great opportunity to carry out my $\mathrm{PhD}$ work in a scientific environment such as that of MPQ.

I am very thankful also to Professor Karl-Ludwig Kompa for funding part of my work in MPQ.

I would like to express my deep gratitude to Werner Fuß for his supervision, guidance and encouragement, with the help of which this work could be successfully carried out. Moreover, his deep scientific knowledge, understanding and experience, as well as his enthusiasm about the experiments and the results throughout these last four years will always constitute a bright example for the future.

I want to express my deep gratitude also to Sergei A. Trushin for all the collaboration and the work in the lab and in the analysis of the results. His valuable experience and knowledge, as well as his interest and enthusiasm made this work possible to achieve.

I am very grateful to Wolfram E. Schmid for helping me in the lab, in the analysis of the results or in any technical issue, as well as for always being open to discussion and helping me every time a question arose, being willing to explain.

I would especially want to thank George D. Tsakiris for his advice, support and encouragement from the first moment I arrived at MPQ.

Many thanks to my good friends Jenny Manolopoulou and Ioannis Daoutidis for their love and all the great time we have spent together these four years in Munich. They have proven so valuable for overcoming all sorts of difficulties and problems in my every-day life and for coming successfully to the end of my $\mathrm{PhD}$.

Many thanks to Tibor Wittmann for being my friend and putting up with my bad mood at the end, during the writing of this thesis. I will never forget the great fun, laughs and long discussions we shared together in the office the last two years.

Many thanks to Elisabeth Magerl for offering her help in writing the "Zusammenfassung" as well as in printing this work. 
Finally, I want to thank my beloved parents, Marios Kosmas and Agni Kosma, for always believing in me, for their precious advice and for offering their caring love and support without ever asking for any exchange. 


\section{List of publications}

Publications included in this thesis are noted with *

* 1. Kyriaki Kosma, Sergei A. Trushin, Werner Fuß, Wolfram E. Schmid Cyclohexadiene ring opening observed with 13 fs resolution: Coherent oscillations confirm the reaction path In preparation

* 2. Kyriaki Kosma, Sergei A. Trushin, Werner Fuß and Wolfram E. Schmid Ultrafast dynamics and coherent oscillations in ethylene excited at $162 \mathbf{~ n m}$ Journal of Physical Chemistry, accepted

* 3. Kyriaki Kosma, Sergei A. Trushin, Wolfram E. Schmid and Werner Fuß Vacuum ultraviolet pulses of $\mathbf{1 1} \mathrm{fs}$ from fifth-harmonic generation of a Ti:sapphire laser Optics Letters 33 (2008) 723

* 4. Kyriaki Kosma, Sergei A. Trushin, Werner Fuß and Wolfram E. Schmid Characterization of the supercontinuum radiation generated by self-focusing of few-cycle 800-nm pulses in argon Journal of Modern Optics, in press

* 5. Sergei A. Trushin, Kyriaki Kosma, Werner Fuß and Wolfram E. Schmid Wavelength-independent ultrafast dynamics and coherent oscillation of a metal-carbon stretch vibration in photodissociation of $\mathrm{Cr}(\mathrm{CO})_{6}$ in the region of $270-345 \mathrm{~nm}$ Chemical Physics, 347 (2008) 309

* 6. Sergei A. Trushin, Kyriaki Kosma, Werner Fuß, and Wolfram E. Schmid Sub-10-fs supercontinuum radiation generated by filamentation of few-cycle $800 \mathrm{~nm}$ pulses in argon Optics Letters 32 (2007) 2432

7. Sergei A. Trushin, Kyriaki Kosma, Werner Fuß, and Wolfram E. Schmid Supercontinuum from a few-cycle filament in argon: characterization and UV spectroscopic appli cation (Proceedings Paper)

Proceedings of SPIE -- Volume No. 6733, 67332W (Jul. 9, 2007)

8. Neset Aközbek, Sergei A. Trushin, Andrius Baltuska, Werner Fuss, Eleftherios Goulielmakis, Kyriaki Kosma, Ferenc Krausz, Subhasis Panja, Matthias Uiberacker, Wolfram E. Schmid, Andreas Becker, Michael Scalora, Mark Bloemer

Extending the supercontinuum spectrum down to $200 \mathbf{~ n m}$ with few-cycle pulses New Journal of Physics 8 (2006) 177

* 9. Sergei A. Trushin, Werner Fuß, Kyriaki Kosma, Wolfram E. Schmid Widely tunable ultraviolet sub-30-fs pulses from supercontinuum for transient spectroscopy Applied Physics B 85 (2006) 1

*10. Sergei A. Trushin, Subhasis Panja, Kyriaki Kosma, Wolfram E. Schmid, Werner Fuß Supercontinuum extending from >1000 to $250 \mathrm{~nm}$, generated by focusing 10-fs laser pulses at 805 nm into Ar Applied Physics B 80 (2005) 399 